\title{
MODELING OF ULTRASONIC AND TERAHERTZ RADIATIONS IN DEFECTIVE TILES FOR CONDITION MONITORING OF THERMAL PROTECTION SYSTEMS
}

by

Ehsan Kabiri Rahani

\author{
A Dissertation Submitted to the Faculty of the \\ Department of Civil Engineering and Engineering Mechanics \\ In Partial Fulfillment of the Requirements \\ For the Degree of \\ DOCTOR OF PHILOSOPHY \\ WITH A MAJOR IN ENGINEERING MECHANICS \\ In the Graduate College
}

THE UNIVERSITY OF ARIZONA

2011 


\section{THE UNIVERSITY OF ARIZONA GRADUATE COLLEGE}

As members of the Dissertation Committee, we certify that we have read the dissertation prepared by Ehsan Kabiri Rahani

entitled Modeling of Ultrasonic and Terahertz Radiations in Defective Tiles for Condition Monitoring of Thermal Protection Systems

and recommend that it be accepted as fulfilling the dissertation requirement for the Degree of Doctor of Philosophy

Date: $11 / 22 / 2011$

Tribikram Kundu

Date: $11 / 22 / 2011$

George Frantziskonis

Date: $11 / 22 / 2011$

Lianyang Zhang

Date:

Final approval and acceptance of this dissertation is contingent upon the candidate's submission of the final copies of the dissertation to the Graduate College.

I hereby certify that I have read this dissertation prepared under my direction and recommend that it be accepted as fulfilling the dissertation requirement.

Dissertation Director: Tribikram Kundu

Date: $11 / 22 / 2011$ 


\section{STATEMENT BY AUTHOR}

This dissertation has been submitted in partial fulfillment of requirements for an advanced degree at the University of Arizona and is deposited in the University Library to be made available to borrowers under rules of the Library.

Brief quotations from this dissertation are allowable without special permission, provided that accurate acknowledgment of source is made. Requests for permission for extended quotation from or reproduction of this manuscript in whole or in part may be granted by the author. 


\section{ACKNOWLEDGEMENTS}

I would like to express my sincere thanks to Professor Tribikram Kundu for his leadership, enthusiasm and motivation during the completion of my $\mathrm{PhD}$ program. Without his kind supports I would not be able to reach the position I am currently standing in. I am also grateful from him offering me precious opportunities to build my future career on a strong foundation. My special thanks also go to Professors George Frantziskonis, Lianyang Zhang and John Kemeny for their support attending my $\mathrm{PhD}$ committee and reviewing the dissertation manuscript. I'm really grateful from Professor Hamid Saadatmanesh advising me in minor field courses and his supports.

Endless thanks with love to my wife Nazanin Babamarandi for her patience during my Master and $\mathrm{PhD}$ program, her endless encourages and I will never forget that she has always been my first emotional supporter and it has always been convincing for me that there is one who accompanies me forever to achieve my planned goals in expense of sacrificing her future missing some great opportunities for herself. Also I will owe her forever rewarding me by giving born to my son Kourosh the most precious asset in my life. My profound love and appreciation goes to my parents Davoud and Effat without them I would never reach here. They have always been the best supporters, friends and motivators. One of the hugest lacks in my life is, not having them beside, during five years staying far from my home country Iran and it deprived me from having their extreme love, beautiful smiles and encouragements. Last but not least I would like to express my deep thanks to my brother Erfan for his continuous support and encouragement and I will never forget the childhood games with him. 
$\tau_{0}$

My beloved wife Nazanin

,

My son, Kourosh the most precious gift from God

$\mathcal{L}$

My mother Effat \& father Davoud dearest to my heart 


\section{TABLE OF CONTENTS}

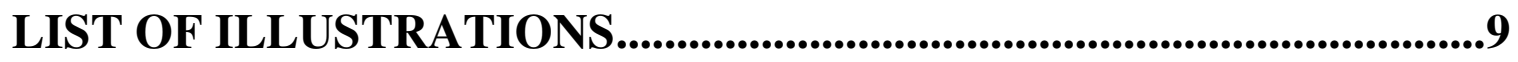

ABSTRACT

CHAPTER 1 THZ RADIATION FOR NDE .....................................23

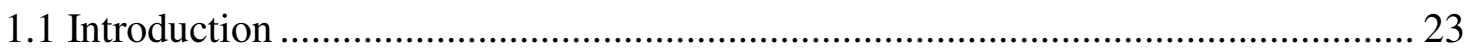

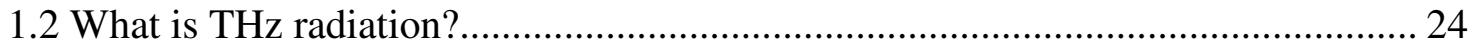

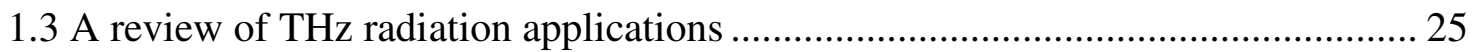

1.4 Introduction to the theory of electromagnetic radiation ........................................ 27

1.4.1 Plane wave equations ..................................................................................... 32

1.4.2 Polarized plane wave and interface interaction ............................................... 34

1.4.3 Coherent THz transmission spectroscopy …………….................................... 38

1.5 Mechanical Damage Detection in Polymer Tiles by THz Radiation ...................... 40

1.5.1 Dielectric material property determination ..................................................... 41

1.5.2 THz Finite Element Analysis ......................................................................... 47

1.5.3 THz-TDs measurement of blocks with mechanical damage .............................. 50

1.6 Heat Induced Damage Detection by THz Radiation ............................................. 56

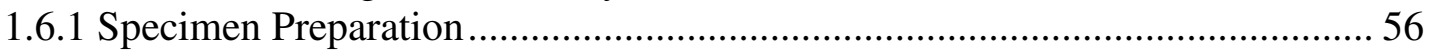

1.6.2 THz-TDs measurement of heat treated blocks .................................................. 57

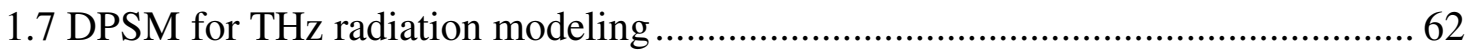

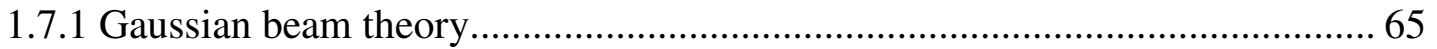

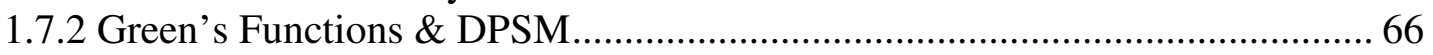

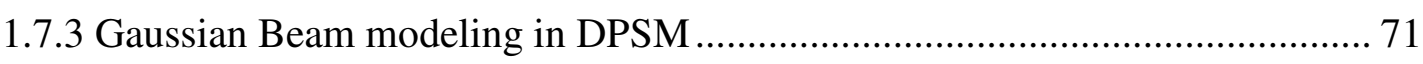

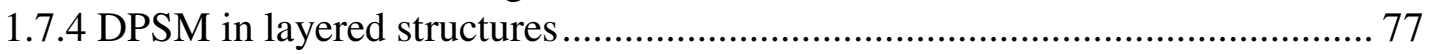

1.7.5 Dielectric scatter modeling in DPSM.......................................................... 81

1.8 Effect of uniform and non-uniform heat damage ............................................... 85

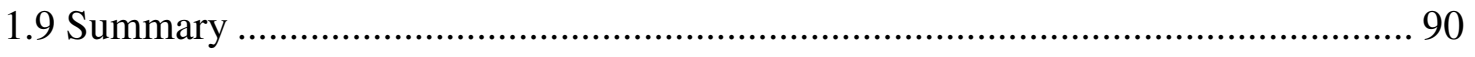

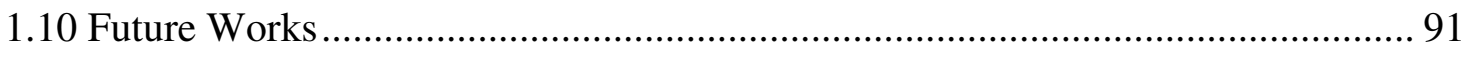




\section{CHAPTER 2 T-DPSM: EXTENSION OF CONVENTIONAL DPSM}

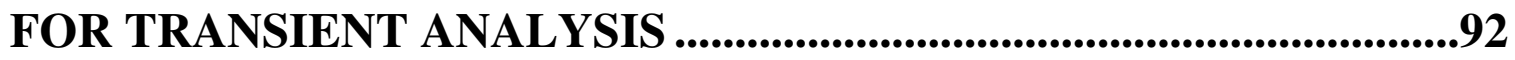

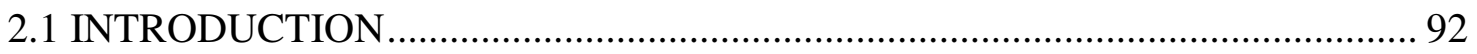

2.2 A Brief Review of Distributed Point Source Method (DPSM)............................. 95

2.3 Matrix Representation for Transducer Cavity Interaction in a Fluid Medium ....... 95

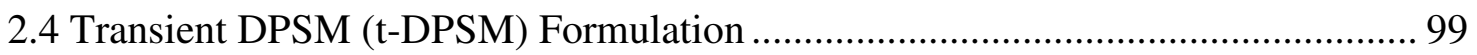

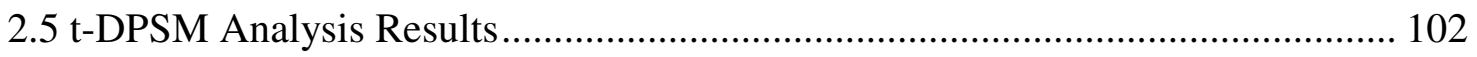

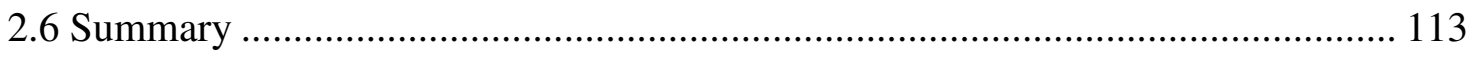

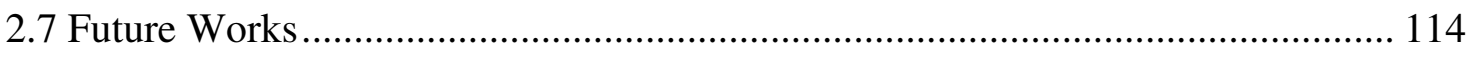

CHAPTER 3 MODIFICATIONS OF DPSM FOR ULTRASONIC AND ELECTROMAGNETIC FIELD MODELING PROBLEMS.................115

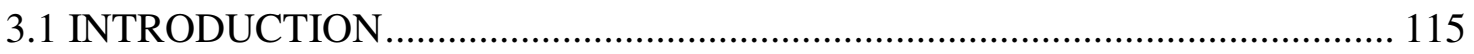

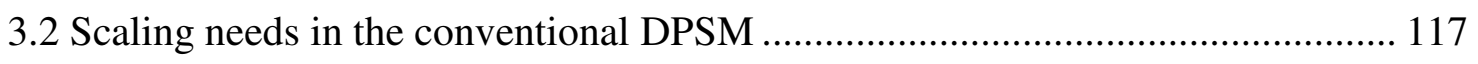

3.3 Proposed Gaussian-DPSM (G-DPSM) method …………………..................... 121

3.4 Proposed Element Source Method (ESM) in Ultrasonic........................................ 123

3.4.1 ESM for modeling ultrasonic transducers ................................................... 128

3.5 Proposed Element Source Method (ESM) in Electromagnetics ........................... 134

3.5.1 ESM Gaussian beam modeling results ............................................................ 138

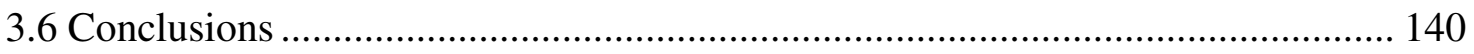

3.7 Future Works ................................................................................................ 141

CHAPTER 4 DPSM FOR SOLVING ACOUSTIC WAVE SCATTERING PROBLEMS .....................................................142

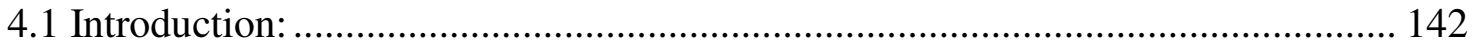

4.2 Advantages of DPSM over FEM in Wave Propagation Problems......................... 144

4.3 DPSM Theoretical Formulation ........................................................................... 149

4.3.1 Computation of displacement and stress Green's functions in the solid........ 150 
4.3.2 Computation of displacement and pressure fields in the fluid ..................... 152

4.3.3 Boundary and Interface Conditions

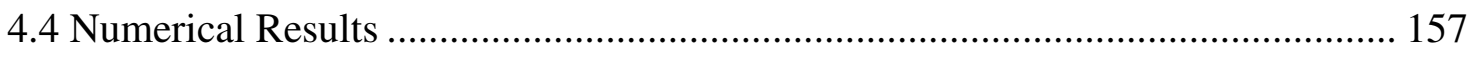

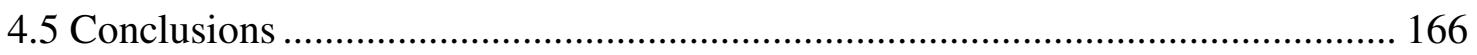

CHAPTER 5 DEVELOPMENT OF THE FORTRAN DPSM3D CODE . .168

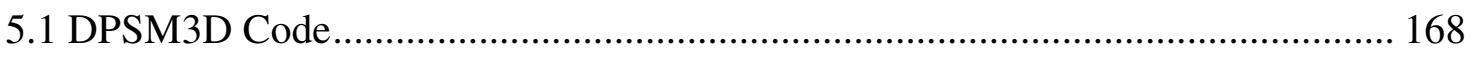

5.2 Case Studies Carried Out by DPSM3D Code ……………………...................... 173

APPENDIX A: DPSM3D FORTRAN SOURCE CODE FOR ULTRASONIC PROBLEMS ..........................................................................176

APPENDIX B: DPSM3D FORTRAN SOURCE CODE FOR ELECTROMAGNETIC PROBLEMS......................................................211

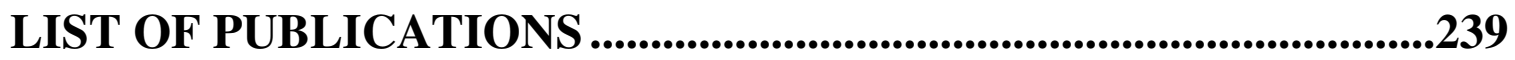

REFERENCES .........................................................................................241 


\section{LIST OF ILLUSTRATIONS}

Figure 1.1 Terahertz band gap in electromagnetic spectrum ................................... 25

Figure 1.2 Schematic for boundary condition at an interface between two media........... 30

Figure 1.3 Obliquely incident plane wave on a plane interface between two dielectric

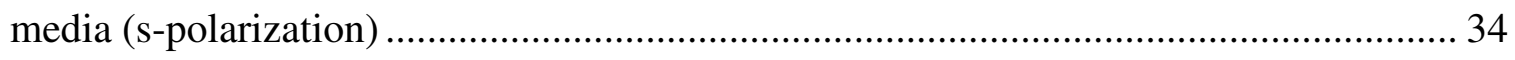

Figure 1.4 Obliquely incident plane wave on a plane interface between two dielectric

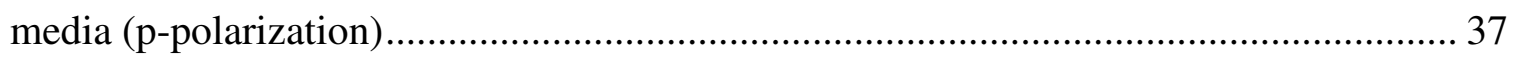

Figure 1.5 Terahertz TDS transmission characterization setup [20]. ........................... 43

Figure 1.6 THz received pulse propagated through free-space and solid foam medium $[21]$ 44

Figure 1.7 Frequency domain response for free-space and solid foam medium [21]...... 45 Figure $1.8 \varepsilon^{\prime}$ (the real part of permittivity index) is plotted with right ordinate and tan $\delta$ (the loss tangent) is plotted with left ordinate both as functions of signal frequency [21].

Figure 1.9 THz Gaussian beam (Freq=400 GHz) passing through a solid foam tile. The beam is propagating in the $\mathrm{x}$-direction from left to right, penetrating through the foam tile placed between the two vertical lines. $e_{z}=$ unit vector normal to the 2D FE model [21]. 48 Figure 1.10 PT value for Reference = air, Sample = solid foam, see equation (1.84) [21].

Figure 1.11 Comparison between experimental and FEM predicted PT values, Reference $=$ air, Sample $=$ solid foam, see equation $(1.84)[21]$ 50 
Figure 1.12 Frequency domain response for T-ray going through a defect-free foam tile and a foam tile with $1 / 8$ inch $(\sim 3 \mathrm{~mm})$ diameter through-hole, as shown in the figure [21].

Figure $1.13 \mathrm{THz}$ beam (Freq=830 GHz) passing through a foam tile with a hole, see Figure 1.12 [21]. 51

Figure 1.14 Frequency domain response for a defect-free foam tile and a foam tile with a $1 / 8$ inch $(\sim 3 \mathrm{~mm})$ diameter hole. Beam propagates parallel to the hole axis [21]. 52

Figure 1.15 PT value for reference signal through the solid foam and sample signal through the defective foam, beam propagates parallel to the hole axis [21]. 53 Figure $1.16 \mathrm{THz}$ beam scattered by a cylindrical hole while propagating parallel to the central axis of the hole - (a) energy distribution, freq $=100 \mathrm{GHz}$; (b) wave fronts, freq = $100 \mathrm{GHz}$; (c) energy distribution, freq = $550 \mathrm{GHz}$; (d) wave fronts, freq $=550 \mathrm{GHz}$; (e) energy distribution, freq $=1000 \mathrm{GHz}$; (f) wave front, freq $=1000 \mathrm{GHz}[21]$. 55 Figure 1.17 (a) Porous material specimen. (b) Heat treatment temperatures for Blocks B1 to $\mathrm{B} 5$ are denoted as $\mathrm{T} 1$ to $\mathrm{T} 5$, respectively [20]. 57

Figure 1.18 Received THz pulse propagated through free-space and Blocks 1, 3 and 5 $[20]$. 58

Figure 1.19 Frequency domain response for the free-space and Blocks 1, 3 and 5 [20].. 59 Figure 1.20 (a) $\varepsilon^{\prime}(\omega)$ (the real part of permittivity index) and (b) $\tan \delta$ (the loss tangent) variations as functions of signal frequency for the five samples [20]. 61

Figure 1.21 Temperature dependence of FIR reflection spectra below100 $\mathrm{cm}^{-1}$ wave number for the $\mathrm{BaTiO}_{3}$ ceramics (from Hoshina et al. [24]) 61 
Figure 1.22 Schematic for modeling a three-medium problem by DPSM - (a) 3D depiction of spherical point sources on both sides of every interface and the lower side of the source, (b) 2D depiction of the DPSM model and the variable names assigned to the interface layers 71

Figure 1.23 Axial electric field in front of a Gaussian beam emitter at $0.5 \mathrm{THz}$ frequency (Analytical solution). 74

Figure 1.24 Axial electric field in front of a truncated Gaussian beam emitter at $0.5 \mathrm{THz}$ frequency (DPSM solution for circular source of radius $3.6 \mathrm{~mm}$ ). 75 Figure 1.25 Axial electric field in front of a truncated Gaussian beam emitter at $0.5 \mathrm{THz}$ frequency (DPSM solution for circular source of radius $4.8 \mathrm{~mm}$ ). 75

Figure 1.26 Comparison between theoretical and DPSM generated electric fields for Gaussian and truncated Gaussian Beams (circular source of radius $=4.8 \mathrm{~mm}$ for DPSM analysis), frequency $=0.5 \mathrm{THz}$ 76 Figure 1.27 Generated Electric Fields for Gaussian distribution of the source strength (frequency $=0.5 \mathrm{THz}$ ): (a) DPSM Solution (circular source of radius $=4.8 \mathrm{~mm}$ ), (b) Theoretical Solution 76 Figure 1.28 Axial electric field in front of a truncated Gaussian beam emitter at $0.75 \mathrm{THz}$ frequency obtained from DPSM modeling (circular source of radius $4.8 \mathrm{~mm}, 29$ Layers of Point Sources are used). 80 Figure 1.29 Comparison between experimental (see ref [21]) and DPSM predicted PT values, Reference medium is air, sample is solid foam. The dashed-dotted curve is 
obtained experimentally while the three dots are generated from the DPSM analyses at three frequencies. 81

Figure 1.30 DPSM modeling and interaction between Gaussian beam and dielectric scatterers; (a) 2D schematic depiction of DPSM model, (b) Gaussian Beam in absence of any scatterer, (c) Gaussian Beam in presence of an off-axis single Scatterer, (d) Gaussian Beam in presence of an on-axis single Scatterer, (e) Gaussian Beam in presence of two scatterers 84

Figure 1.31 Schematic of samples for different case studies with DPSM (' $h$ ' is the sample thickness). 86

Figure 1.32 Schematic for modeling a four-layered structure with DPSM 87 Figure 1.33 Gaussian beam field generated by DPSM (emitter radius $=7 \mathrm{~mm}$, frequency $=0.22 \mathrm{THz}$ ); (a): Case A, (b): Case B, (c): Case C and (d): Case D of Figure 1.31, all dimensions are in meters. 88

Figure 1.34 Gaussian beam generated axial electric field obtained from DPSM modeling, comparison between Cases A, B and C (emitter and interface radius $=7 \mathrm{~mm}$, Frequency $=0.22 \mathrm{THz}$. 89 Figure 1.35 Gaussian beam generated axial PT values obtained from DPSM modeling, comparison between Cases A, B, C and D of Figure 1.31 (emitter and interface radius $=7$ $\mathrm{mm}$, Frequency $=0.22 \mathrm{THz}$. 89 Figure 2.1 DPSM model for a spherical cavity inside a fluid containing a point-focused ultrasonic transducer. 96 
Figure 2.2 A time dependent point source strength function by linear interpolation; (b): Point source schematic 100

Figure 2.3 Pressure field (at $t=4 \mu \mathrm{s}$ ) generated by a flat circular transducer of diameter 4 mm, (a): t-DPSM, (b): FEM (COMSOL). Numbers along horizontal and vertical axes are in meters. . Note that the center of the transducer is located at the origin. 103

Figure 2.4 Pressure field (at $t=9 \mu \mathrm{s}$ ) generated by a flat circular transducer of diameter 4 mm, (a): t-DPSM, (b): FEM (COMSOL). Numbers along horizontal and vertical axes are in meters. Note that the center of the transducer is located at the origin 103 Figure 2.5 Pressure time history (at $\mathrm{r}=0 \mathrm{~mm}, \mathrm{z}=4 \mathrm{~mm}$ ) generated by a flat circular transducer of diameter $4 \mathrm{~mm}$, (a): t-DPSM, (b): FEM (COMSOL). Time is in second, pressure units are arbitrary - $\mathrm{Pa}$ or psi, same as that in equation (2.16)..... 105

Figure 2.6 Pressure time history (at $\mathrm{r}=4 \mathrm{~mm}, \mathrm{z}=6 \mathrm{~mm}$ ) generated by a flat circular transducer of diameter $4 \mathrm{~mm}$, (a): t-DPSM, (b): FEM (COMSOL). Time is in second, pressure units are arbitrary - $\mathrm{Pa}$ or psi, same as that in equation (2.16).... 106 Figure 2.7 Pressure field (at $t=7 \mu \mathrm{s}$ ) generated by a point focused transducer (radius $=3$ mm, opening angle $\left.=90^{\circ}\right),(a)$ : t-DPSM, (b): FEM (COMSOL) 107 Figure 2.8 Pressure time history (at $\mathrm{r}=0 \mathrm{~mm}, \mathrm{z}=4 \mathrm{~mm}$ ) generated by a point focused transducer (radius $=3 \mathrm{~mm}$, theta $\left.=90^{\circ}\right) ;(\mathrm{a})$ : t-DPSM, (b): FEM (COMSOL). Time is in second, pressure units are arbitrary - Pa or psi, same as that in equation (2.16) 108 Figure 2.9 t-DPSM computed pressure fields (at $t=6.9 \mu \mathrm{s}$ ) for a point focused transducer (radius $=3 \mathrm{~mm}$, opeing angle $\left.=90^{\circ}\right)$. Pressure fields are generated for the case with no cavity (image (c)) and in presence of a spherical cavity of diameter $0.3 \mathrm{~mm}$ with 
horizontal eccentricity $=0.0 \mathrm{~mm}$ (image (a)) and with horizontal eccentricity $=0.5 \mathrm{~mm}$ (image (b)). 109

Figure 2.10 Reflected signal strength or energy (from a spherical cavity of $0.3 \mathrm{~mm}$ diameter placed at the focal plane) as a function of time for a point focused transducer (radius $=3 \mathrm{~mm}$, opening angle $=90^{\circ}$ ) for different horizontal eccentricities. Time is in second, the energy has arbitrary unit that depends on the unit of equation (2.16).

Figure 2.11 Reflected signal strength or energy (from a spherical cavity of $0.3 \mathrm{~mm}$ diameter placed on the transducer axis of symmetry) as a function of time for a point focused transducer (radius $=3 \mathrm{~mm}$, opening angle $=90^{\circ}$ ) for three different locations of the cavity - cavity eccentricities are zero (cavity is placed at the focal point), $-0.3 \mathrm{~mm}$ (cavity is moved from the focal point by $0.3 \mathrm{~mm}$ towards the transducer) and $+0.3 \mathrm{~mm}$ (moved away from the transducer). Time is in second, the energy has arbitrary unit that depends on the unit of equation (2.16).

Figure 2.12 Reflected signal strength or energy (from two spherical cavities of $0.3 \mathrm{~mm}$ diameter) as a function of time for a point focused transducer (radius $=3 \mathrm{~mm}$, opening angle $=90^{\circ}$ ), one cavity is located at the focal point and the second cavity is placed at four different locations in $(\mathrm{r}, \mathrm{z})$ coordinates. Time is in second, the energy has arbitrary unit that depends on the unit of equation (2.16). 112 Figure 3.1 Ultrasonic field modeling by DPSM - superimposing the contributions of M source points distributed over the transducer face the ultrasonic field at $\mathrm{N}$ target points can be obtained [28] 118 
Figure 3.2 Variation of the normal velocity on the transducer surface obtained from the conventional DPSM analysis. Peak value of 1 is obtained at 499 points on the transducer surface where small spheres (see Figure 3.1) corresponding to 499 point sources touch the transducer face. Lower velocity values in between these peaks make the average normal velocity of the transducer surface less than 1 [see [28]]. 118 Figure 3.3 Comparison between theoretical and DPSM (with and without scaling) generated pressure fields along the central axis of a circular transducer of $2 \mathrm{~mm}$ diameter vibrating at $5 \mathrm{MHz}$ frequency

Figure 3.4 Schematic depiction of Parent and Fictitious (child) Gaussian Point sources 120

Figure 3.5 Velocity distribution on the transducer surface, Gaussian DPSM, $2 \mathrm{~mm}$ diameter transducer, Frequency $=5 \mathrm{MHz}$ : (a) when $a=r_{s}$ (b) when $a=0.5 r_{s}$ 121 Figure 3.6 Comparison between theoretical and Gaussian DPSM $\left(a=0.5 \mathrm{r}_{\mathrm{s}}\right)$, generated pressure fields on the central axis of a circular transducer of $2 \mathrm{~mm}$ diameter vibrating at 5 $\mathrm{MHz}$ frequency 122

Figure 3.7 (a) Schematic depiction of a source element in global coordinate (b) Main element and the sub-elements in local coordinate 124 Figure 3.8 Nodal source strength variation: $1.55 \mathrm{~mm} \times 1.55 \mathrm{~mm}$ transducer, uniform velocity boundary condition 129

Figure 3.9 Axial pressure variation: $1.55 \mathrm{~mm} \times 1.55 \mathrm{~mm}$ transducer vibrating at $5 \mathrm{MHz}$ : Theory - continuous line; ESM (30x30 elements) for uniform velocity boundary condition - dotted line 130 
Figure 3.10 Modified boundary condition; $1.55 \mathrm{~mm} \times 1.55 \mathrm{~mm}$ transducer vibrating at 5 $\mathrm{MHz}$ 131

Figure 3.11 Axial pressure variation: $1.55 \mathrm{~mm} \times 1.55 \mathrm{~mm}$ transducer vibrating at $5 \mathrm{MHz}$ : continuous line - Theory; other curves - ESM predictions with modified boundary conditions when divided into $28 \times 28,20 \times 20,10 \times 10$ and $5 \times 5$ elements 131

Figure 3.12 Axial pressure variation: $1.55 \mathrm{~mm} \times 1.55 \mathrm{~mm}$ transducer vibrating at $5 \mathrm{MHz}$ : continuous line - Theory; other curves show DPSM predictions, generated by $34 \times 34$, $28 \times 28,20 \times 20$ and $10 \times 10$ point sources

Figure 3.13 Axial Pressure variation: $1.55 \mathrm{~mm} \times 1.55 \mathrm{~mm}$ transducer vibrating at $5 \mathrm{MHz}$ - computed results by ESM (20x20 element sources) and DPSM (34×34 point sources) are compared with the theoretical curve 133 Figure 3.14 Gaussian Beam axial Electric Field at $0.5 \mathrm{THz}$ frequency (Comparison between DPSM and Analytical solution) 134

Figure 3.15 (a) Schematic depiction of a source element in global coordinate (b) Main element and the sub-elements in local coordinate 135

Figure 3.16 Meshing the $4.8 \mathrm{~mm}$ radius transducer by triangular elements and Gaussian Electric field boundary condition on surface 139

Figure 3.17 Gaussian Beam axial Electric Field at $0.5 \mathrm{THz}$ frequency (Comparison between ESM and Analytical solution). 140

Figure 4.1 Pressure field variation along the central axis of a flat circular transducer : Comparison between the theoretical curve (dotted line), finite element solution (dashed 
line) and DPSM solution (continuous line). The scale for the vertical axis is Pascal for transducer surface velocity amplitude $1 \mu \mathrm{m} / \mathrm{s}$ (see [28]). 145

Figure 4.2 Pressure field variation along the central axis of a concave point focused circular transducer : Comparison between the theoretical curve (dotted line), finite element solution (dashed line) and DPSM solution (continuous line). The radius of curvature of the transducer is $4 \mathrm{~mm}$ and it forms a $40^{\circ}$ angle at the center of curvature. All three curves coincide. The scale for the vertical axis is Pascal for transducer surface velocity amplitude $1 \mu \mathrm{m} / \mathrm{s}$ (see [28]). 146

Figure 4.3 Pressure field variation along the central axis of a flat square transducer: Comparison between the RSI solution (dotted line), finite element solution (dashed line) and DPSM solution (continuous line). The transducer is $0.5 \mathrm{~mm} \times 0.5 \mathrm{~mm}$ and the signal frequency is $5 \mathrm{MHz}$. Note that the DPSM matches better with the RSI solution. The Scale for the vertical axis is Pascals for transducer surface velocity amplitude $1 \mu \mathrm{m} / \mathrm{s}$ (see [28]).

Figure 4.4 Pressure field variation along the central axis of a flat square transducer: comparison between the RSI solution (dotted line), finite element solution (dashed line), and DPSM solution (continuous line). The transducer is $1.55 \times 1.55 \mathrm{~mm}$ and the signal frequency is $5 \mathrm{M} \mathrm{Hz}$. A fourth curve (dashed-dotted line) is generated by 2-D finite element model for a $1.5-\mathrm{mm}$-wide transducer. Neither the 2-D nor 3-D finite element model produces the RSI solution. The scale for the vertical axis is pascals for transducer surface velocity amplitude $1 \mu \mathrm{m} / \mathrm{s}$. (b) Pressure field variation along the central axis of a flat square $(1.55 \times 1.55 \mathrm{~mm})$ transducer: as in $(\mathrm{a})$, except that the computation is carried 
out only up to $3.5 \mathrm{~mm}$ in the axial direction instead of $10 \mathrm{~mm}$ to have much smaller finite elements. The 3-D finite element analysis now shows better matching with the RSI and DPSM solutions compared with that in (a). The scale for the vertical axis is pascals for transducer surface velocity amplitude $1 \mu \mathrm{m} / \mathrm{s}$ (see [28]). 148

Figure 4.5 (color online) Problem geometry showing the distribution of point sources (small circles) along the acoustic lens (or transducer), the cavity and the interface between the solid and fluid media (see [90]). 154

Figure 4.6 Comparison between the DPSM results with the approximate theoretical solution for a cavity in Plexiglas (radius of the cavity $=0.16 \mathrm{~mm}$, cavity center from the solid-fluid interface $=4.8 \mathrm{~mm}$, radius of the lens $=2 \mathrm{~cm}$, lens angle $=60^{\circ}$, Freq $=1.5$ $\mathrm{MHz})($ see [90]). 160

Figure 4.7 Reflected force (force felt by the acoustic lens due to the reflected ultrasonic signal) versus the horizontal distance between the lens center (the center of curvature of the lens) and the cavity center when there is only one cavity of radius $0.2 \mathrm{~mm}$ present in the solid medium (Glass-Quartz). Distance of the cavity center from the fluid-solid interface $=0.5 \mathrm{~mm}$, the center of curvature of the lens is at a depth of $0.3 \mathrm{~mm}$ inside the solid (see [90]). 161

Figure 4.8 Reflected energy generated force vs. the horizontal distance between the lens center (the center of curvature of the lens) and the cavity center when there is only one cavity of radius $0.2 \mathrm{~mm}$ present in the solid medium. Distance of the cavity center from the fluid-solid interface $=0.5 \mathrm{~mm}$, the lens center is at a depth of $0.5 \mathrm{~mm}$ inside the solid (see [90]). 162 
Figure 4.9 Reflected energy generated force as a function of the horizontal distance between the lens center and the cavity center when there is only one cavity present in the solid medium. Cavity radius is $0.2 \mathrm{~mm}$ (solid line) and $0.3 \mathrm{~mm}$ (dashed line), cavity center distance from the fluid-solid interface is $1 \mathrm{~mm}$, distance of the lens center (the center of curvature of the lens) from the interface is $0.5 \mathrm{~mm}$. High oscillations near the peaks of the curve are noted. Figure 4.11 is plotted to investigate this high oscillation phenomenon more closely (see [90]). 163

Figure 4.10 Reflected energy generated force as a function of the horizontal distance of the lens center, from the cavity center (for the single cavity) or from the midpoint of the two cavities (for the double cavities), distance of the fluid-solid interface is $1 \mathrm{~mm}$ from the cavity center and $0 \mathrm{~mm}$ from the lens center. High oscillations near the peaks in some curves are noted. Figure 4.11 is plotted to investigate this high oscillation phenomenon more closely (see [90]) 165

Figure 4.11 Reflected force vs. the horizontal distance of the lens center, from the cavity center (for the single cavity case) or from the midpoint of the two cavities (for the double cavity case), distance of the fluid-solid interface from the cavity center is $1 \mathrm{~mm}$ and from

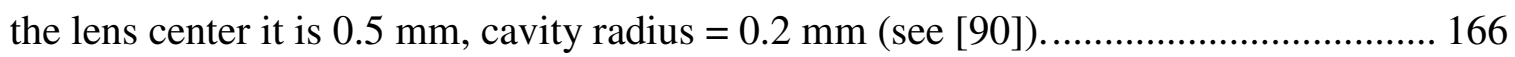

Figure 5.1 Different types of problem analyzed by DPSM3D code............................ 169 Figure 5.2 The objects oriented structure of interfaces in DPSM3D.......................... 169

Figure 5.3 Defining a Polygon interface object in DPSM3D ................................... 170

Figure 5.4 Schematic of a DPSM model composed of interface objects....................... 171 
Figure 5.5 Computed pressure fields in front of the acoustic lens in the presence of two small cavities of radius $=0.1 \mathrm{~mm}$ as the distance between the two cavities is varied from $0.8 \mathrm{~mm}$ to $2 \mathrm{~mm}$ : (a) $0.8 \mathrm{~mm}$, (b) $1.2 \mathrm{~mm}$, (c) $1.6 \mathrm{~mm}$, and (d) $2.0 \mathrm{~mm}$. Numbers along the horizontal and vertical axes are given in meters and denote positions of the field points in a Cartesian coordinate system whose origin coincides with the bottom left corner of the figure. Scales are slightly different in the x- and y-directions (see [85]). . 174 Figure 5.6 Normalized force sensed by the acoustic lens when the cavities move horizontally on the focal plane. The signal frequency $=1 \mathrm{MHz}$. Distance denotes the horizontal distance from the focal point to the center of the cavity (for the single-cavity case) or the center of the right cavity (for the two-cavity case). Distance between the cavity centers is $1.2 \mathrm{~mm}$ (see [85]) 175 


\begin{abstract}
Condition based monitoring of Thermal Protection Systems (TPS) is necessary for safe operations of space shuttles when quick turn-around time is desired. In the current research Terahertz radiation (T-ray) has been used to detect mechanical and heat induced damages in TPS tiles. Voids and cracks inside the foam tile are denoted as mechanical damage while property changes due to long and short term exposures of tiles to high heat are denoted as heat induced damage.

Ultrasonic waves cannot detect cracks and voids inside the tile because the tile material (silica foam) has high attenuation for ultrasonic energy. Instead, electromagnetic terahertz radiation can easily penetrate into the foam material and detect the internal voids although this electromagnetic radiation finds it difficult to detect delaminations between the foam tile and the substrate plate. Thus these two technologies are complementary to each other for TPS inspection.

Ultrasonic and T-ray field modeling in free and mounted tiles with different types of mechanical and thermal damages has been the focus of this research. Shortcomings and limitations of FEM method in modeling 3D problems especially at high-frequencies has been discussed and a newly developed semi-analytical technique called Distributed Point Source Method (DPSM) has been used for this purpose.

A FORTRAN code called DPSM3D has been developed to model both ultrasonic and electromagnetic problems using the conventional DPSM method. This code is designed in a general form capable of modeling a variety of geometries. DPSM has been extended from ultrasonic applications to electromagnetic to model THz Gaussian beams,
\end{abstract}


multilayered dielectrics and Gaussian beam-scatterer interaction problems. Since the conventional DPSM has some drawbacks, to overcome it two modification methods called G-DPSM and ESM have been proposed.

The conventional DPSM in the past was only capable of solving time harmonic (frequency domain) problems. Time history was obtained by FFT (Fast Fourier Transform) algorithm. In this research DPSM has been extended to model DPSM transient problems without using FFT. This modified technique has been denoted as tDPSM.

Using DPSM, scattering of focused ultrasonic fields by single and multiple cavities in fluid \& solid media is studied. It is investigated when two cavities in close proximity can be distinguished and when it is not possible. A comparison between the radiation forces generated by the ultrasonic energies reflected from two small cavities versus a single big cavity is also carried out. 


\section{CHAPTER 1}

\section{THZ RADIATION FOR NDE}

\subsection{Introduction}

In recent years, the terahertz (THz or T-ray) technology (see [1]) has received a lot of attention because of its unique properties and capabilities that make it very attractive as a non-destructive evaluation (NDE) tool. This chapter covers some NDE applications of $\mathrm{THz}$ radiation - focusing on the detection of mechanical and heat induced anomalies in porous media as well as $\mathrm{THz}$ radiation modeling. In many NDE applications $\mathrm{THz}$ radiation is more suitable than ultrasonic energy. For example ultrasonic energy cannot propagate through ceramic foam tiles used in the space shuttle Thermal Protection Systems (TPS). Therefore, internal defects in these tiles cannot be detected by ultrasonic signals. TPS shows primarily three types of mechanical damage 1) delaminations at the tile-substrate interface, 2) delaminations between different layers within the tile and 3) voids and cracks inside the foam tile. First type defect can be detected by ultrasonic signals by propagating guided waves through the substrate medium but second and third type damages are difficult to detect by the ultrasonic signal because it cannot propagate very well through such porous medium. Besides these mechanical defects another type of damage that can occur in TPS tiles due to heat exposure is the change in material properties inside the tile. This damage can be called heat induced damage or heat damage. Heat damage caused by high heat exposures of tiles has not been studied extensively. In this chapter through experimental and analytical investigations it 
is demonstrated that $\mathrm{THz}$ is capable of detecting the mechanical and heat induced damages inside porous foam tiles.

\subsection{What is $\mathrm{THz}$ radiation?}

Terahertz $(\mathrm{THz})$ radiation is electromagnetic radiation with frequency range lying between the microwave and infrared regions of the spectrum (see Figure 1.1). A $\mathrm{THz}$ wave is not observable by naked eyes. The part of $\mathrm{THz}$ range shared with Infrared region can be felt, warming the human skin. Terahertz radiation is non-ionizing sub-millimeter microwave radiation and shares with microwaves the capability to penetrate a wide variety of non-conducting materials. Terahertz radiation can pass through clothing, paper, cardboard, wood, masonry, plastic and ceramics. It can also penetrate fog and clouds, but cannot penetrate metal or water. Since the Earth's atmosphere is a strong absorber of terahertz radiation, the range of terahertz radiation is quite short and it is not useful for communications. In addition, producing and detecting coherent terahertz radiation was technically challenging until the 1990s. The least explored region mainly due to the technical difficulties involved in making efficient and compact $\mathrm{THz}$ sources and detectors. The lack of suitable technologies led to the $\mathrm{THz}$ band being called the " $\mathrm{THz}$ gap”. This technological gap has been rapidly diminishing in the last two decades (see [2]) . 


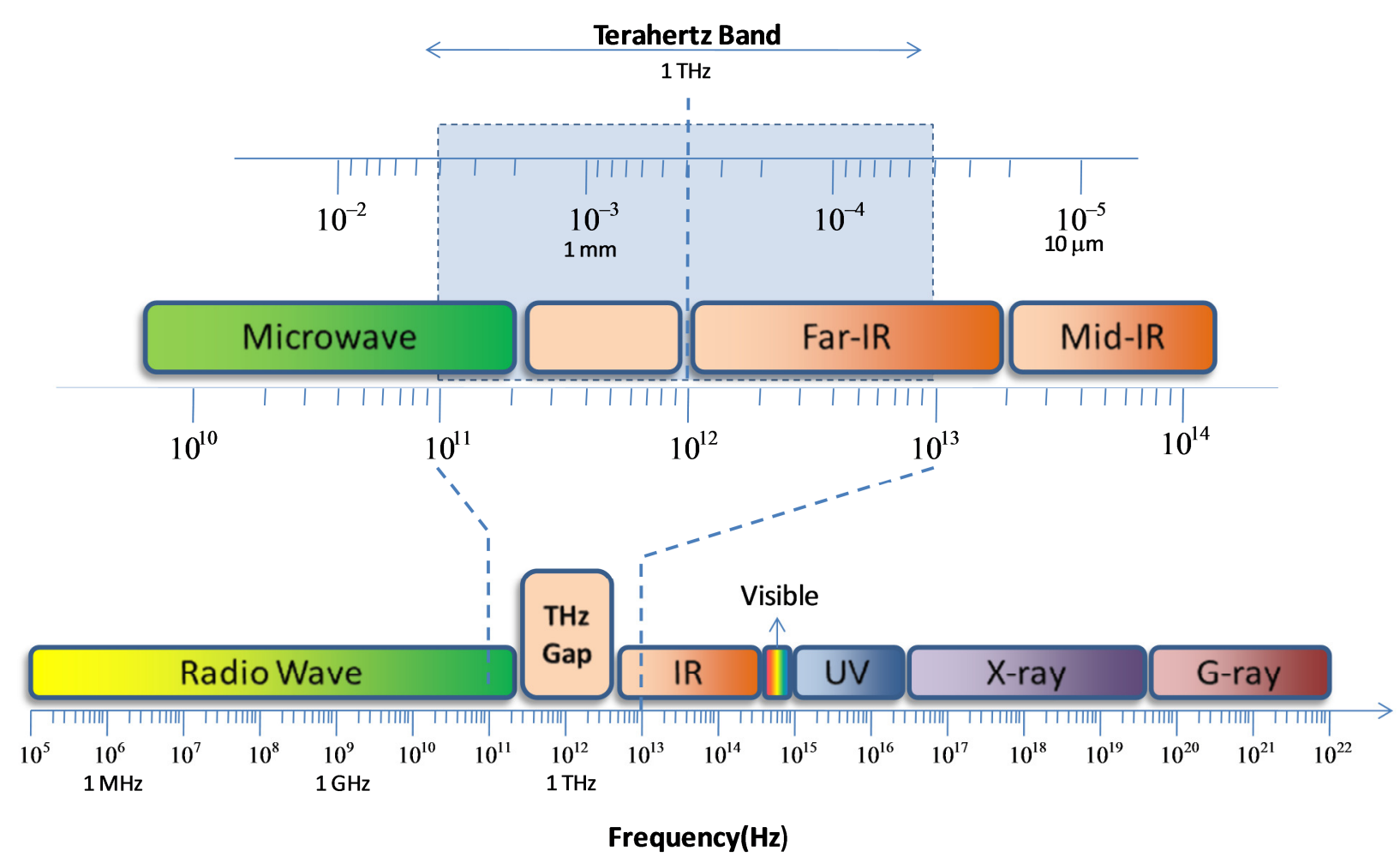

Figure 1.1 Terahertz band gap in electromagnetic spectrum

\subsection{A review of THz radiation applications}

Sanchez et al. [3] investigated THz imaging and sensing techniques for damage and defect assessment in insulating foam and carbon fiber materials. For the carbon fiber samples, both $\mathrm{THz}$ imaging at $0.6 \mathrm{THz}$ and the time-domain data were used to evaluate the extent of heat damage. It was shown that the carbon fiber has a polarization dependent reflectivity in the $\mathrm{THz}$ frequency range, which can be related to burn damage level. Stoik et al. [4] assessed THz-TDS as a nondestructive evaluation technique for aircraft composites. They inspected damage to glass fiber composites including voids, delaminations, mechanical damage, and heat induced damage. They concluded that it would be difficult to detect heat induced damage by $\mathrm{THz}$ technology because burning and 
localized heat damage did not cause noticeable change in the refractive index or absorption coefficient of T-ray. Zhao et al. [5] presented THz TDS measurements on three kinds of polystyrene foam that demonstrate that the material has an extremely low refractive index and a low absorption in the range from 0.1 to $4.0 \mathrm{THz}$. They showed that polystyrene foam is an excellent material for use as a filter to block near-IR light while transmitting $\mathrm{THz}$ light, and that it can be used as a sample substrate for $\mathrm{THz}$ imaging. Piesiewicz et al. [6] measured the frequency dependent refractive index and absorption coefficient of a variety of common building and plastic materials between 100 and 1000 GHz range. Zhong et al. [7] have investigated $\mathrm{THz}$ time-of-flight tomographic imaging in nondestructive identification of foam insulation of space shuttle fuel tanks, with prefabricated defects. Zhong et al. [8] have reported detection of space shuttle insulation foam defects using a $0.2 \mathrm{THz}$ Gunn diode oscillator as the light source, and a pyroelectric camera as the detector. The size and location of the defects have been identified [9] by tomographic T-ray imaging in which $\mathrm{THz}$ pulses are reflected from refractive-index discontinuities inside an object, The time delays of these pulses are used to determine the positions of the discontinuities along the propagation direction. Karpowicz et al. [10] reported the use of a compact continuous-wave sub-terahertz system for inspection applications using electronic generation and detection methods. They presented examples of the measurement of NASA's insulating panels and applicability of the technology to other nondestructive testing applications. Zhang et al. [11] reported the sensing of explosive materials and illicit drugs by using terahertz time-domain spectroscopy (THzTDS). They showed that the pulsed THz-TDS and CW (continuous wave) imaging 
system have the ability to inspect the manmade or natural defects in aerospace materials. THz imaging and spectroscopy technique are also found to be promising solutions to the problem of identifying some illicit drugs, explosives, and damage in some composite materials. In Reference [12] quantitative assessment protocol has been developed to evaluate potential signal processing methods for THz NDE of spray-on foam insulation. In Banks and Gibson's [13] study the location of the Knit lines in foam blocks have been predicted using numerical simulations. Instead of using dispersion models or treating the refraction index as frequency dependent, they assumed this index to be constant for the value associated with the dominant frequency mode of the interrogating pulse. In the work of Anastasi and Madaras [14] Terahertz NDE imaging under paint for corrosion inspection has been examined. Oyama et al. [15] developed a compact sub-Terahertz imaging system with a 0.2 THz-band GaAs TUNNETT diode oscillator and studied its application for NDE of timbers, concrete and ceramic tiles.

\subsection{Introduction to the theory of electromagnetic radiation}

Four Maxwell equations are fundamental of electromagnetic wave propagation theory and describe all classical electromagnetic phenomena:

$$
\begin{gathered}
\text { (i) } \nabla . \mathbf{D}=\rho_{f} \quad \text { or } \nabla . \mathbf{E}=\frac{\rho_{t}}{\varepsilon_{0}} \quad \text { (Gauss's Law) } \\
\text { (ii) } \nabla . \mathbf{B}=0 \\
\text { (iii) } \nabla \times \mathbf{E}=-\frac{\partial \mathbf{B}}{\partial t} \quad \text { (Faraday's Law) } \\
\text { (iv) } \nabla \times \mathbf{H}=\mathbf{J}_{f}+\frac{\partial \mathbf{D}}{\partial t} \quad \text { (Ampere's Law) }
\end{gathered}
$$


In which:

$$
\begin{gathered}
\mathbf{D}=\varepsilon_{0} \mathbf{E}+\mathbf{P} \\
\rho_{t}=\rho_{f}+\rho_{b} \\
\rho_{b}=-\nabla . \mathbf{P} \\
\mathbf{H}=\frac{\mathbf{B}}{\mu_{0}}-\mathbf{M}
\end{gathered}
$$

D : Electric Displacement (electrical flux density), Columb/meter ${ }^{2}$ or $\mathrm{C} / \mathrm{m}^{2}$

E : Electrical Field, N/C

P : Polarization Density, $\mathrm{C} / \mathrm{m}^{2}$

$\rho_{f}:$ Free charge density, $\mathrm{C} / \mathrm{m}^{3}$

$\rho_{b}$ : Bound charge density, $\mathrm{C} / \mathrm{m}^{3}$

$\rho_{t}:$ Total charge density, $\mathrm{C} / \mathrm{m}^{3}$

$\varepsilon_{0}:$ Permittivity of Free Space $=8.85 \times 10^{-12} \mathrm{C}^{2} / \mathrm{N} . \mathrm{m}^{2}$

M : Magnetization Density, A/m

H: Magnetic Field, A/m

B : Magnetic flux density, N/(A.m)

$\mu_{0}$ : Permeability of Free Space $=4 \pi \times 10^{-7} \mathrm{~N} / \mathrm{A}^{2}$

For linear materials:

$$
\mathbf{P}=\varepsilon_{0} \chi_{\mathrm{e}} \mathbf{E}
$$




$$
\begin{gathered}
\mathbf{D}=\varepsilon \mathbf{E} \\
\varepsilon=\varepsilon_{0}\left(1+\chi_{\mathrm{e}}\right) \\
\mu=\mu_{0}\left(1+\chi_{\mathrm{m}}\right) \\
\mathbf{B}=\mu \mathbf{H}
\end{gathered}
$$

$\chi_{e}$ : Electric Susceptibility of the Medium, Dimensionless

$\varepsilon$ : Permittivity of the Linear Medium, $\mathrm{C}^{2} / \mathrm{N} \cdot \mathrm{m}^{2}$

$\mu$ : Permeability of Linear Medium, N/A ${ }^{2}$

$\chi_{\mathrm{m}}:$ Magnetic Susceptibility of the Medium, Dimensionless

The interface boundary conditions between two media (Figure 1.2) are given as:

$$
\begin{gathered}
\left(\mathbf{D}_{1}-\mathbf{D}_{2}\right) \cdot \mathbf{n}=\sigma_{f} \text { or } \mathrm{D}_{1 \perp}-\mathrm{D}_{2 \perp}=\sigma_{f} \\
\left(\mathbf{B}_{1}-\mathbf{B}_{2}\right) \cdot \mathbf{n}=0 \text { or } \mathrm{B}_{1 \perp}-\mathrm{B}_{2 \perp}=\mathbf{0} \\
\mathbf{E}_{1 \mid}-\mathbf{E}_{2 \|}=\mathbf{0} \text { or } \mathbf{n} \times\left(\mathbf{E}_{1}-\mathbf{E}_{2}\right)=\mathbf{0} \\
\mathbf{H}_{1 \mid}-\mathbf{H}_{2 \|}=\mathbf{J}_{\mathrm{sf}} \times \mathbf{n} \text { or } \mathbf{n} \times\left(\mathbf{H}_{1}-\mathbf{H}_{2}\right)=\mathbf{J}_{\mathrm{fs}}
\end{gathered}
$$

In which:

$\sigma_{f}=$ Surface charge density, $\mathrm{C} / \mathrm{m}^{2}$

$\mathbf{J}_{\mathrm{fs}}=$ Free Surface current density vector, $\mathrm{C} /(\mathrm{m} . \mathrm{s})$ 


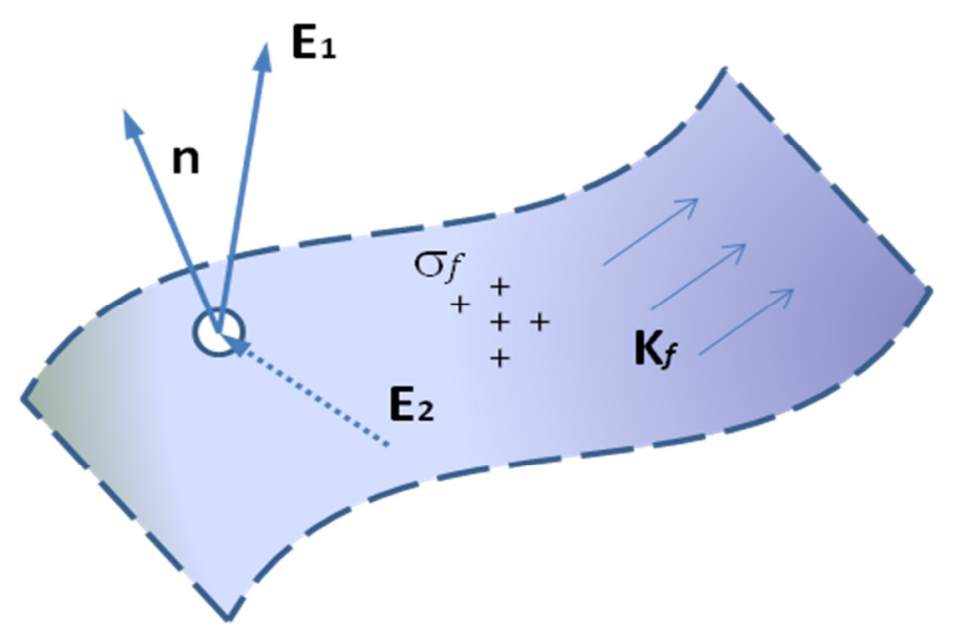

Figure 1.2 Schematic for boundary condition at an interface between two media

For linear materials Equations (1.14) and (1.15) can be written as follows:

$$
\begin{gathered}
\varepsilon_{1} \mathrm{E}_{1 \perp}-\varepsilon_{2} \mathrm{E}_{2 \perp}=\sigma_{f} \\
\left(\mu_{1} \mathbf{H}_{1}-\mu_{2} \mathbf{H}_{2}\right) \cdot \mathbf{n}=0 \text { or } \mu_{1} \mathrm{H}_{1 \perp}-\mu_{2} \mathrm{H}_{2 \perp}=\mathbf{0}
\end{gathered}
$$

When there is no free charge or free current on the surface equation (1.17) becomes:

$$
\mathbf{H}_{1 \mid}-\mathbf{H}_{2 \|}=\mathbf{0} \text { or } \mathbf{n} \times\left(\mathbf{H}_{1}-\mathbf{H}_{2}\right)=\mathbf{0}
$$

From Ohm's law the free current density is given as a function of the electric field:

$$
\mathbf{J}_{f}=\sigma \mathbf{E}
$$

It is assumed that there is no source current density in the domain otherwise a term $\mathbf{J}_{s}$ should be added to $\mathbf{J}_{f}$.

For a linear medium when there is no free charge or free current in domain: 


$$
\begin{gathered}
\nabla . \mathbf{E}=0 \quad(\text { Gauss's Law }) \\
\nabla \cdot \mathbf{H}=0 \\
\nabla \times \mathbf{E}=-\mu \frac{\partial \mathbf{H}}{\partial t} \quad(\text { Faraday's Law }) \\
\nabla \times \mathbf{H}=\sigma \mathbf{E}+\varepsilon \frac{\partial \mathbf{E}}{\partial t} \text { (Ampere's Law) }
\end{gathered}
$$

By applying the curl to equations (1.24) and (1.25), and using equations (1.22) and (1.23) we get the classical wave equations:

$$
\begin{aligned}
& \nabla^{2} \mathbf{E}-\mu \varepsilon \frac{\partial^{2} \mathbf{E}}{\partial t^{2}}-\mu \sigma \frac{\partial \mathbf{E}}{\partial t}=0 \\
& \nabla^{2} \mathbf{H}-\mu \varepsilon \frac{\partial^{2} \mathbf{H}}{\partial t^{2}}-\mu \sigma \frac{\partial \mathbf{H}}{\partial t}=0
\end{aligned}
$$

The refraction index " $n$ " for a medium is defined as:

$$
n=c / v
$$

In which:

$$
v=\frac{1}{\sqrt{\mu \varepsilon}}, \quad c=\frac{1}{\sqrt{\mu_{0} \varepsilon_{0}}}
$$

$v:$ Electromagnetic wave speed in medium $c:$ Electromagnetic wave speed in vacuum 


\subsubsection{Plane wave equations}

Harmonic time-dependent plane waves (monochromatic waves) are special solutions of wave equations (1.26) and (1.27):

$$
\begin{aligned}
& \mathbf{E}(\mathbf{r}, \mathrm{t})=\mathbf{E}_{0} e^{i(\mathbf{k} \cdot \mathbf{r}-\omega t)} \\
& \mathbf{H}(\mathbf{r}, \mathrm{t})=\mathbf{H}_{0} e^{i(\mathbf{k} . \mathbf{r}-\omega t)}
\end{aligned}
$$

In which $\mathbf{k}$ is the wave vector with direction of plane wave propagation $\mathbf{n}$, its magnitude $k$ is the wave number. By substituting equations (1.30) and (1.31) in wave equations (1.26) and (1.27) one gets

$$
k^{2}=\mu \varepsilon \omega^{2}+i \mu \sigma \omega
$$

Since $k$ is a complex number, separating the real and imaginary parts gives:

$$
\begin{gathered}
k=k_{r}+i k_{i} \\
k_{r}=\omega \sqrt{\frac{\mu \varepsilon}{2}}\left[\sqrt{1+\left(\frac{\sigma}{\varepsilon \omega}\right)^{2}}+1\right]^{1 / 2} \\
k_{i}=\omega \sqrt{\frac{\mu \varepsilon}{2}}\left[\sqrt{1+\left(\frac{\sigma}{\varepsilon \omega}\right)^{2}}-1\right]^{1 / 2}
\end{gathered}
$$

The imaginary part of $k$ results in attenuation (decreasing the wave amplitude with propagation length) of the electromagnetic wave. In non-dispersive medium ( $\varepsilon$ is not complex) when the material is non-conductive $(\sigma=0)$ the imaginary part $k_{i}$ is zero and the medium has no attenuation. "Skin depth" in conductive materials is the distance 
from the surface where the amplitude of the wave in Eqs. (1.30) and (1.31) reduces by a factor of $1 / e$ :

$$
\delta_{\text {skin }}=\frac{1}{k_{i}}
$$

For dielectric (non-conductive) materials equation (1.33) is simplified to the form:

$$
k=\omega \sqrt{\mu \varepsilon}=\frac{n \omega}{c}
$$

Inserting equations (1.30) and (1.31) in equations (1.3) and (1.23) we get

$$
\begin{aligned}
\mathbf{k} \cdot \mathbf{E} & =0 \\
\mathbf{k} \cdot \mathbf{H} & =0
\end{aligned}
$$

It proves that the electric and magnetic field vectors are transverse to the wave propagation direction $\mathbf{n}$ (or k). Using plane wave equations (1.30) and (1.31) and inserting them into equation (1.25) gives:

$$
\begin{gathered}
\nabla \times \mathbf{E}=i k(\mathbf{n} \times \mathbf{E})=-\mu \frac{\partial \mathbf{H}}{\partial t}=\mu i \omega \mathbf{H} \\
\mathbf{H}=\frac{k}{\mu \omega}(\mathbf{n} \times \mathbf{E})=\frac{1}{\eta}(\mathbf{n} \times \mathbf{E}) \\
\mathbf{H}=\frac{1}{\eta}\left(\mathbf{n} \times \mathbf{E}_{0}\right) e^{i(\mathbf{k} \cdot \mathbf{r}-\omega t)}
\end{gathered}
$$

In which $\eta$ is defined as intrinsic impedance of the medium as follows:

$$
\eta=\sqrt{\frac{\mu}{\varepsilon}}
$$


From the above equations we can conclude that for a plane electromagnetic (EM) wave the Electric and Magnetic vectors are perpendicular to each other at every point in the domain.

\subsubsection{Polarized plane wave and interface interaction}

Figure 1.3 depicts the incident, reflected and transmitted waves on a plane of incidence for s-polarized (the case in which polarization of incident wave is perpendicular to the plane of incidence). From Equation (1.30) we have:
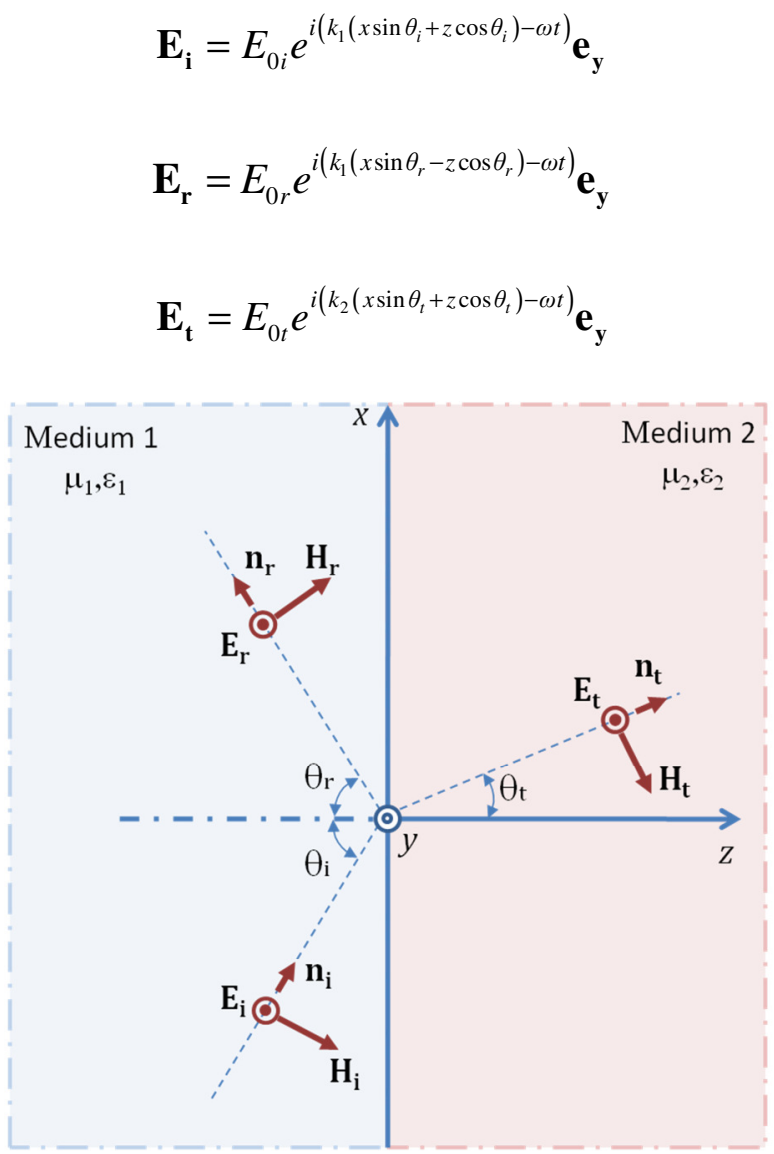

Figure 1.3 Obliquely incident plane wave on a plane interface between two dielectric media (s-polarization) 
From equations (1.42), (1.44), (1.45) and (1.46) we get

$$
\begin{aligned}
& \mathbf{H}_{\mathbf{i}}=\frac{E_{0 i}}{\eta_{1}} e^{i\left(k_{1}\left(x \sin \theta_{i}+z \cos \theta_{i}\right)-\omega t\right)}\left(\sin \theta_{i} \mathbf{e}_{\mathbf{z}}-\cos \theta_{i} \mathbf{e}_{\mathbf{x}}\right) \\
& \mathbf{H}_{\mathbf{r}}=\frac{E_{0 r}}{\eta_{1}} e^{i\left(k_{1}\left(x \sin \theta_{r}-z \cos \theta_{r}\right)-\omega t\right)}\left(\sin \theta_{r} \mathbf{e}_{\mathbf{z}}+\cos \theta_{r} \mathbf{e}_{\mathbf{x}}\right) \\
& \mathbf{H}_{\mathbf{t}}=\frac{E_{0 t}}{\eta_{2}} e^{i\left(k_{2}\left(x \sin \theta_{t}+z \cos \theta_{t}\right)-\omega t\right)}\left(\sin \theta_{t} \mathbf{e}_{\mathbf{z}}-\cos \theta_{t} \mathbf{e}_{\mathbf{x}}\right)
\end{aligned}
$$

From the boundary conditions in equations (1.16) and (1.20) the tangential components of $\mathbf{E}$ and $\mathbf{H}$ should be continuous across the interface at $\mathrm{z}=0$ that results in:

$$
\begin{gathered}
E_{0 i} e^{i\left(k_{1} x \sin \theta_{i}\right)}+E_{0 r} e^{i\left(k_{1} x \sin \theta_{r}\right)}=E_{0 t} e^{i\left(k_{2} x \sin \theta_{t}\right)} \\
\frac{E_{0 r}}{\eta_{1}} \cos \theta_{r} e^{i\left(k_{1} x \sin \theta_{r}\right)}-\frac{E_{0 i}}{\eta_{1}} \cos \theta_{i} e^{i\left(k_{1} x \sin \theta_{i}\right)}=-\frac{E_{0 t}}{\eta_{2}} \cos \theta_{t} e^{i\left(k_{2} x \sin \theta_{t}\right)}
\end{gathered}
$$

In order to satisfy these equations for all $x$ values we should have

$$
\begin{gathered}
k_{1} \sin \theta_{i}=k_{1} \sin \theta_{r}=k_{2} \sin \theta_{t} \\
E_{0 i}+E_{0 r}=E_{0 t} \\
\theta_{i}=\theta_{r} \\
\frac{1}{\eta_{1}}\left(E_{0 i}-E_{0 r}\right) \cos \theta_{i}=\frac{E_{0 t}}{\eta_{2}} \cos \theta_{t}
\end{gathered}
$$

For non-conducting materials from equations (1.52), (1.28) and (1.32) the Snell's law is obtained as: 


$$
\begin{gathered}
n_{1} \sin \theta_{i}=n_{2} \sin \theta_{t} \\
t_{r, s}=\frac{E_{0 r}}{E_{0 i}}=\frac{\eta_{2} \cos \theta_{i}-\eta_{1} \cos \theta_{t}}{\eta_{2} \cos \theta_{i}+\eta_{1} \cos \theta_{t}}=\frac{\left(\frac{\eta_{2}}{\eta_{1}}\right) \cos \theta_{i}-\sqrt{1-\frac{n_{1}}{n_{2}} \sin ^{2} \theta_{i}}}{\left(\frac{\eta_{2}}{\eta_{1}}\right) \cos \theta_{i}+\sqrt{1-\frac{n_{1}}{n_{2}} \sin ^{2} \theta_{i}}}
\end{gathered}
$$

where, $t_{r, s}$ is s-polarization reflection coefficient

$$
t_{t, s}=\frac{E_{0 t}}{E_{0 i}}=\frac{2 \eta_{2} \cos \theta_{i}}{\eta_{2} \cos \theta_{i}+\eta_{1} \cos \theta_{t}}=\frac{2\left(\frac{\eta_{2}}{\eta_{1}}\right) \cos \theta_{i}}{\left(\frac{\eta_{2}}{\eta_{1}}\right) \cos \theta_{i}+\sqrt{1-\frac{n_{1}}{n_{2}} \sin ^{2} \theta_{i}}}
$$

$t_{t, s}$ is s-polarization transmission coefficient

Figure 1.4 depicts the incident, reflected and transmitted waves on a plane interface for $\mathrm{p}$ polarized (the case in which polarization of incident wave is parallel to the plane of incidence). From Equation (1.30) we have

$$
\begin{aligned}
& \mathbf{E}_{\mathbf{i}}=E_{0 i} e^{i\left(k_{1}\left(x \sin \theta_{i}+z \cos \theta_{i}\right)-\omega t\right)}\left(-\sin \theta_{i} \mathbf{e}_{\mathbf{z}}+\cos \theta_{i} \mathbf{e}_{\mathbf{x}}\right) \\
& \mathbf{E}_{\mathbf{r}}=E_{0 r} e^{i\left(k_{1}\left(x \sin \theta_{r}-z \cos \theta_{r}\right)-\omega t\right)}\left(\sin \theta_{r} \mathbf{e}_{\mathbf{z}}+\cos \theta_{r} \mathbf{e}_{\mathbf{x}}\right) \\
& \mathbf{E}_{\mathbf{t}}=E_{0 t} e^{i\left(k_{2}\left(x \sin \theta_{t}+z \cos \theta_{t}\right)-\omega t\right)}\left(-\sin \theta_{t} \mathbf{e}_{\mathbf{z}}+\cos \theta_{t} \mathbf{e}_{\mathbf{x}}\right)
\end{aligned}
$$

Using equations (1.42), (1.59), (1.60) and (1.61) we get:

$$
\mathbf{H}_{\mathbf{i}}=\frac{E_{0 i}}{\eta_{1}} e^{i\left(k_{1}\left(x \sin \theta_{i}+z \cos \theta_{i}\right)-\omega t\right)} \mathbf{e}_{\mathbf{y}}
$$




$$
\begin{gathered}
\mathbf{H}_{\mathbf{r}}=-\frac{E_{0 r}}{\eta_{1}} e^{i\left(k_{1}\left(x \sin \theta_{r}-z \cos \theta_{r}\right)-\omega t\right)} \mathbf{e}_{\mathbf{y}} \\
\mathbf{H}_{\mathbf{t}}=\frac{E_{0 t}}{\eta_{2}} e^{i\left(k_{2}\left(x \sin \theta_{t}+z \cos \theta_{t}\right)-\omega t\right)} \mathbf{e}_{\mathbf{y}}
\end{gathered}
$$

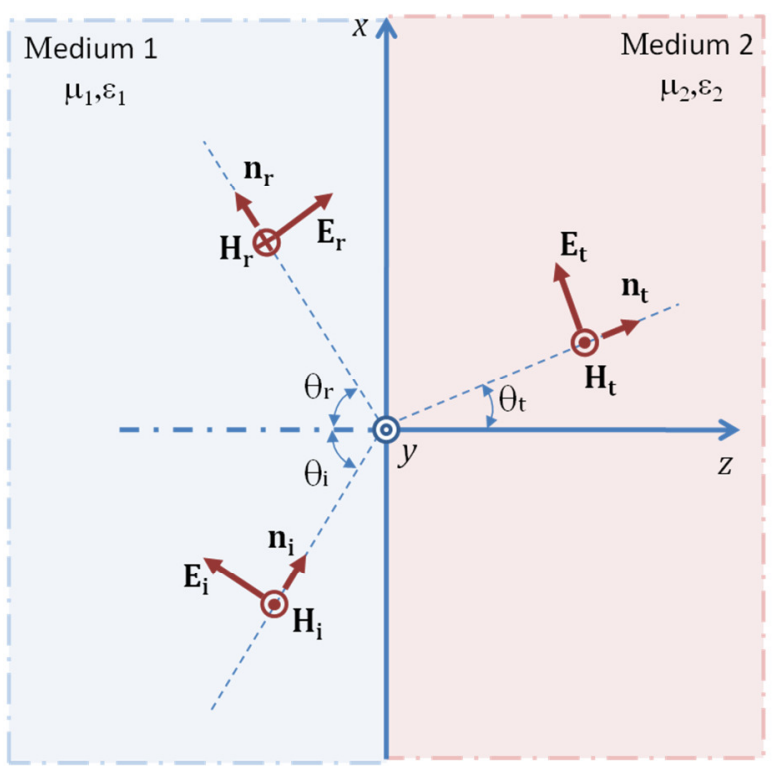

Figure 1.4 Obliquely incident plane wave on a plane interface between two dielectric media (p-polarization)

Similar to s-polarization case the boundary condition in equations (1.16) and (1.20) at interface $\mathrm{z}=0$ gives:

$$
\begin{gathered}
\frac{E_{0 i}}{\eta_{1}} e^{i\left(k_{1} x \sin \theta_{i}\right)}-\frac{E_{0 r}}{\eta_{1}} e^{i\left(k_{1} x \sin \theta_{r}\right)}=\frac{E_{0 t}}{\eta_{2}} e^{i\left(k_{2} x \sin \theta_{t}\right)} \\
E_{0 r} \cos \theta_{r} e^{i\left(k_{1} x \sin \theta_{r}\right)}+E_{0 i} \cos \theta_{i} e^{i\left(k_{1} x \sin \theta_{i}\right)}=E_{0 t} \cos \theta_{t} e^{i\left(k_{2} x \sin \theta_{t}\right)}
\end{gathered}
$$

Validity of the above equations for all $\mathrm{x}$ values gives:

$$
\begin{gathered}
k_{1} \sin \theta_{i}=k_{1} \sin \theta_{r}=k_{2} \sin \theta_{t} \\
\theta_{i}=\theta_{r}
\end{gathered}
$$




$$
\begin{gathered}
\frac{1}{\eta_{1}}\left(E_{0 i}-E_{0 r}\right)=\frac{E_{0 t}}{\eta_{2}} \\
\left(E_{0 i}+E_{0 r}\right) \cos \theta_{i}=E_{0 t} \cos \theta_{t}
\end{gathered}
$$

For non-conducting materials from equations (1.67), (1.28) and (1.32) the Snell's law as in equation (1.56) is obtained.

$$
t_{r, p}=\frac{E_{0 r}}{E_{0 i}}=\frac{\eta_{2} \cos \theta_{t}-\eta_{1} \cos \theta_{i}}{\eta_{2} \cos \theta_{t}+\eta_{1} \cos \theta_{i}}=\frac{\sqrt{1-\frac{n_{1}}{n_{2}} \sin ^{2} \theta_{i}}-\left(\frac{\eta_{1}}{\eta_{2}}\right) \cos \theta_{i}}{\sqrt{1-\frac{n_{1}}{n_{2}} \sin ^{2} \theta_{i}}+\left(\frac{\eta_{1}}{\eta_{2}}\right) \cos \theta_{i}}
$$

$t_{r, p}$ is p-polarization reflection coefficient

$$
t_{t, p}=\frac{E_{0 t}}{E_{0 i}}=\frac{2 \eta_{2} \cos \theta_{i}}{\eta_{2} \cos \theta_{t}+\eta_{1} \cos \theta_{i}}=\frac{2 \cos \theta_{i}}{\sqrt{1-\frac{n_{1}}{n_{2}} \sin ^{2} \theta_{i}}+\left(\frac{\eta_{1}}{\eta_{2}}\right) \cos \theta_{i}}
$$

$t_{t, p}$ is p-polarization transmission coefficient

\subsubsection{Coherent THz transmission spectroscopy}

Coherent $\mathrm{THz}$ spectroscopy is a technique commonly used to measure dielectric properties of materials. By coherent detection scheme measuring both amplitude and phase of $\mathrm{THz}$ fields, simultaneous determination of real and imaginary parts of dielectric constants is possible [1].

Dielectric properties are frequency dependent in dispersive materials and can be described by its complex permittivity $\varepsilon(\omega)$ and complex permeability $\mu(\omega)$ as follows: 


$$
\begin{aligned}
& \varepsilon(\omega)=\varepsilon^{\prime}(\omega)-i \varepsilon^{\prime \prime}(\omega) \\
& \mu(\omega)=\mu^{\prime}(\omega)-i \mu^{\prime \prime}(\omega)
\end{aligned}
$$

$\varepsilon_{\mathrm{r}}(\omega)$ and $\mu_{\mathrm{r}}(\omega)$ are the relative permittivity and permeability, respectively defined as the ratio of the permittivity and permeability of a substance to that of free space or vacuum.

Microwave engineers commonly use loss tangent ( $\tan \delta$ ) [16] (a convenient parameter representing the loss in a dielectric material) defined as:

$$
\tan \delta=\varepsilon^{\prime \prime}(\omega) / \varepsilon^{\prime}(\omega)
$$

In transmission $\mathrm{THz}$ spectroscopy a polarized beam in vacuum is incident normally on a homogeneous layer of material with thickness " $d$ ", passes through its thickness and is then received on the other side by a $\mathrm{THz}$ detector.

Using equations $(1.57),(1.58),(1.72)$ and (1.73) for $\theta_{i}=0$ the reflection and transmission factors for both p-polarized and s-polarized equations give the simplified forms as shown below:

$$
\begin{aligned}
t_{r} & =\frac{\eta_{\text {sample }}-1}{\eta_{\text {sample }}+1} \\
t_{t l} & =\frac{2 \eta_{\text {sample }}}{\eta_{\text {sample }}+1} \\
t_{t l} & =\frac{2}{\eta_{\text {sample }}+1}
\end{aligned}
$$


In which $\eta_{\text {sample }}$ is the relative intrinsic impedance of sample, $t_{r}$ is the reflection coefficient from sample to vacuum, and $t_{t, l}$ is the transmission coefficient from vacuum to sample and $t_{t, 2}$ is the transmission coefficient from sample to vacuum.

The total transmitted field considering the multiple Fabry-Perot reflection is determined by superposition of all transmitted parts as follows:

$$
E_{t}=E_{i} t_{t, 1} t_{t, 2} e^{i \phi_{d}}+E_{i} t_{t, 1} t_{t, 2} t_{r} t_{r} e^{2 i \phi_{d}}+\ldots=E_{i} t_{t, 1} t_{t, 2} \sum_{m=0}^{\infty}\left(t_{r} t_{r} e^{2 i \phi_{d}}\right)^{m}=\frac{E_{i} t_{t, 1} t_{t, 2} e^{i \phi_{d}}}{1-t_{r} t_{r} e^{2 i \phi_{d}}}
$$

In which $\phi_{d}$ is the phase shift:

$$
\phi_{d}=n_{\text {sample }} \omega \frac{d}{c}
$$

Finally the total transmission coefficient from equation (1.79) gives:

$$
t_{\text {total }}=\frac{E_{t}}{E_{i}}=\frac{t_{t, 1} t_{t, 2} e^{i \phi_{d}}}{1-t_{r} t_{r} e^{2 i \phi_{d}}}
$$

\subsection{Mechanical Damage Detection in Polymer Tiles by THz Radiation}

This section describes how pulsed $\mathrm{THz}$ radiation technology can be used to detect mechanical damages in polymer tiles. It is divided into two parts, the first part deals with finding the dielectric material properties of the foam material. The numerical FE (finite element) analysis can be carried out to verify the experimental results. In Part II the foam samples are subjected to mechanical damages by drilling a hole inside the sample. The sample is subjected to $\mathrm{THz}$ pulses from different directions both perpendicular and parallel to the hole-axis. The received response for the damaged tile is compared with the 
reference signal for the solid foam specimen. The interaction between the $\mathrm{THz}$ beam and the mechanical defect are investigated experimentally as well as through numerical modeling.

\subsubsection{Dielectric material property determination}

In order to carry out the numerical analysis the first step is to have a good estimate of the material properties. The electromagnetic properties of a material can be described by its complex relative permittivity, $\varepsilon=\varepsilon^{\prime}-i \varepsilon^{\prime \prime}$, and complex relative permeability, $\mu=\mu^{\prime}-i \mu^{\prime \prime} \mu=\mu^{\prime}-i \mu^{\prime \prime}$, in which the imaginary parts represent losses. Since no magnetic behavior is expected from the foam sample, its permeability $\mu=1$. The complex dielectric constants composed of real and imaginary parts, $\varepsilon$ and $\varepsilon^{\prime \prime}$ are found first. Dielectric constants (see equations (1.73) and (1.74)) are dispersive or frequency dependent [11]:

Since the sample's permeability coefficient $\mu=1$ one transmission experiment is enough to measure its dielectric properties ([17-19]). In THz-TDS transmission measurement the sample is placed on the $\mathrm{THz}$ pulse beam path between the transmitter and the receiver. The transmitted signal through the sample is recorded in time domain and denoted as the sample scan. A reference scan is then taken without the sample on the beam path. The ratio between the sample and reference spectra would be the calibrated sample transmission coefficient T. Because the measurement is coherent, both the magnitude and the phase of $\mathrm{T}$ are obtained. Pulsed-THz is preferable over continuous 
wave $(\mathrm{CW})$ because measuring phase at single $\mathrm{THz}$ frequency is very hard or nonexistent for current $\mathrm{THz} \mathrm{CW}$ techniques.

The transmitted signal through a sample slab that is optically thick enough and has low loss, contains a series of pulses separated in time, corresponding to different orders of Fabry-Perot reflections in the sample. For example, in the case of normal incidence, the zero-th order transmission coefficient $T_{0}$, and the first-order coefficient $T_{1}$ due to one Fabry-Perot reflection, can be expressed as :

$$
\begin{gathered}
T_{0}(n)=\frac{4 n}{(n+1)^{2}} \exp [2 \pi i(1-n) f d / c] \\
T_{1}(n)=T_{0}(n)\left(\frac{n-1}{n+1}\right)^{2} \exp [-4 \pi i n f d / c]
\end{gathered}
$$

Equation (1.82) is derived from (1.79) neglecting the higher order reflections. In Equation (1.82) $f$ is frequency, $d$ is the sample thickness, $c$ is the speed of light, and $n$ is the refractive index of the sample. Because the foam sample has a refractive index close to 1 (free-space) the first-order term $T_{1}$ and all higher order terms are close to zero, and thus indistinguishable as separated pulses in the time-domain output waveform. Therefore, the sample transmitted signal spectrum, summation of all orders of FabryPerot reflection terms, should converge after certain orders of terms are included in the summation. In the data extraction, it is found that after the 2 nd term is included, the solution converges. The rest of the extraction is simply fitting the term $T_{0}+T_{1}$ with the measured complex transmission coefficient numerically, and determining the value of 
complex $n$ at every frequency value ([19]). Once the refractive index is extracted, the sample permittivity $\varepsilon$ is obtained as $\varepsilon=n^{2}$.

The samples used to carry out the experiment are typical packing foams cut into three-dimensional brick specimens with dimensions $1 \times 1 \times 0.5$ in $(2.54 \times 2.54 \times 1.27$ $\mathrm{mm})$.

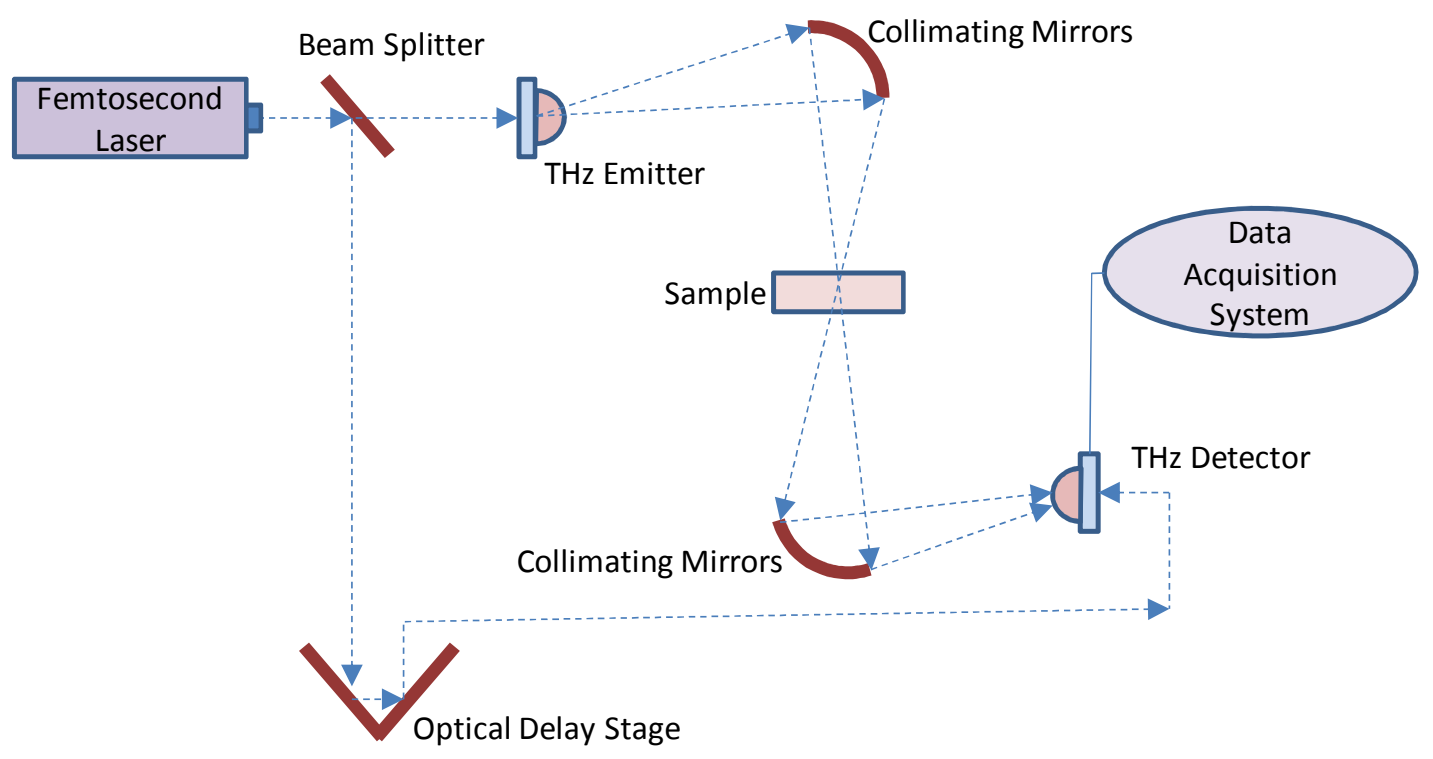

Figure 1.5 Terahertz TDS transmission characterization setup [20].

A photoconductive terahertz TDS system from Advanced Photonix Inc. is used. A terahertz pulse is generated by biased coplanar lines on a low-temperature-grown GaAs substrate, excited by a femtosecond laser. The detector is a $5 \mu m$ gap dipole antenna, which is also fabricated on a low temperature-grown GaAs substrate. One part of the same femtosecond laser pulse is guided to the detector through an optical delay line as the gating signal for recording the received terahertz waveform. The specimen is placed on the path of the terahertz pulse beam between the emitter and the detector. Figure 1.5 shows the transmission measurement configuration. Because the measurement is 
coherent, both the magnitude and the phase of the sample response can be obtained. The TDS used here has a bandwidth spanning from $50 \mathrm{GHz}$ to $1.2 \mathrm{THz}$ with pulse truncated 25 ps before the pulse peak and 83 ps after the peak. The transmitted signal through the sample is recorded in the time domain and denoted as the sample scan (see Figure 1.6). A reference scan is then taken without the sample on the beam path. The ratio between the sample and reference spectra is the calibrated sample transmission coefficient $\mathrm{T}$.

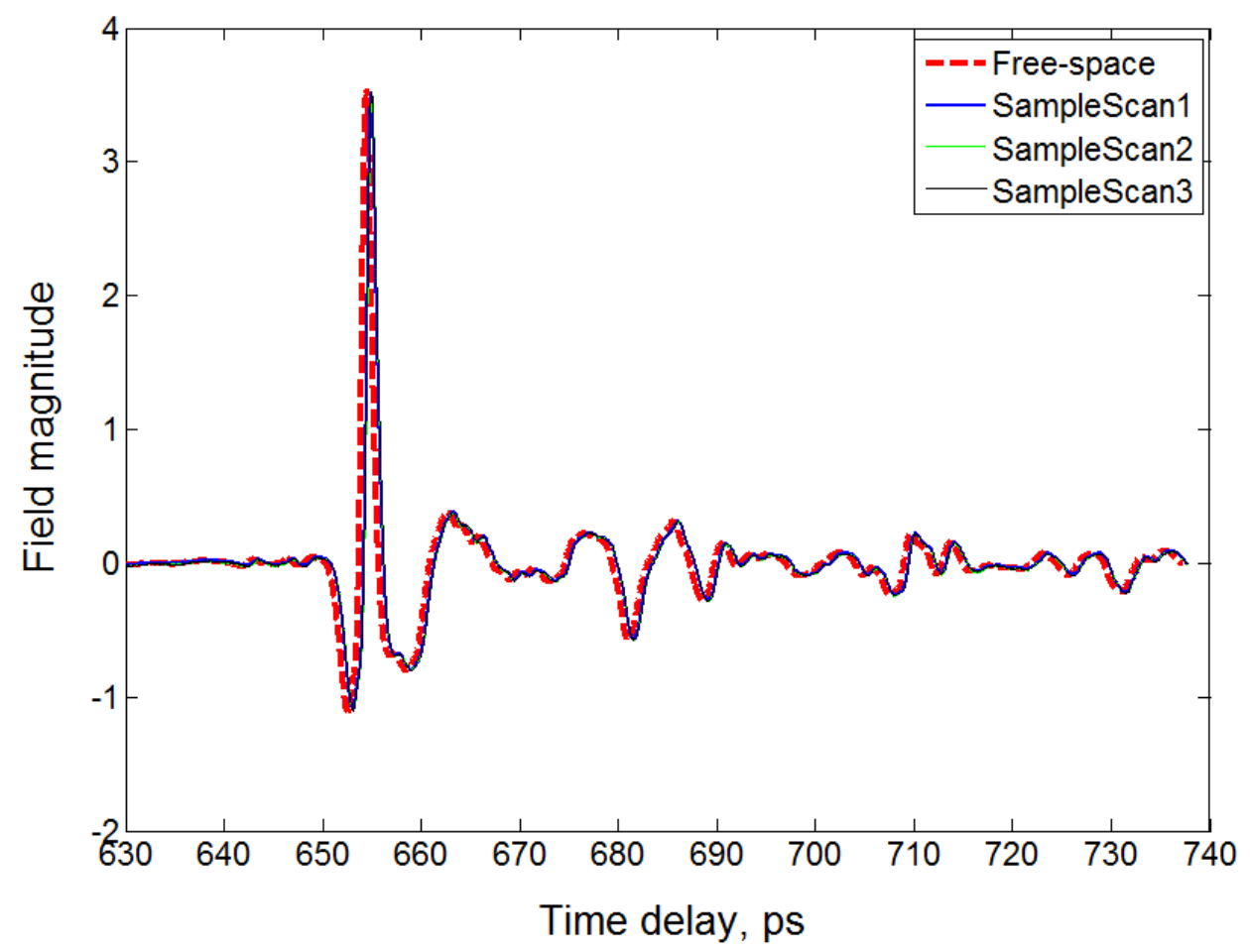

Figure 1.6 THz received pulse propagated through free-space and solid foam medium [21].

The sample is scanned three times to minimize the possible experimental errors. In order to calculate the dielectric constants, the time domain electric field responses are converted to frequency-domain. As depicted in Figure 1.7, in the frequency-domain response of the received pulse there is only a small difference between the signal passing 
through the air (the reference signal) and through the foam sample (the sample signal). It is evident that at lower frequencies below $400 \mathrm{GHz}$ the reference and sample signals coincide so the material behaves almost like air, but at higher frequencies (near THz) the sample signal lies slightly below the reference signal.

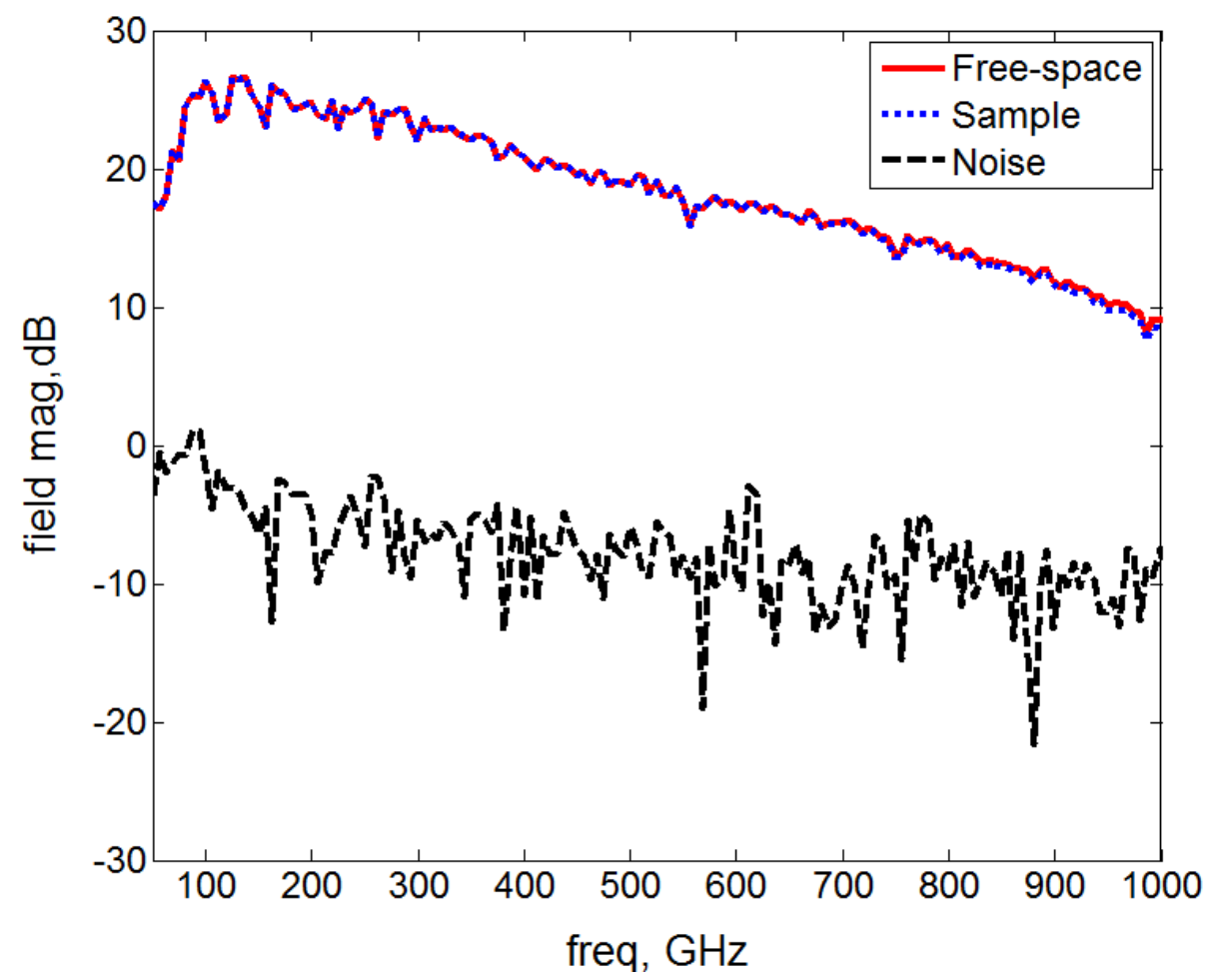

Figure 1.7 Frequency domain response for free-space and solid foam medium [21].

In order to magnify the distinction between reference and sample signals the power transmittance factor is used which is given bellow:

$$
P T(\omega)=20 \times \log _{10}\left(\frac{E S(\omega)}{E R(\omega)}\right)
$$

In which:

$\mathrm{PT}=$ Power transmittance in $\mathrm{dB}$ 
$\mathrm{ER}=$ Reference (air) electric field response in Fourier domain

ES $=$ Sample electric field response in Fourier domain

Dielectric constants $\varepsilon^{\prime}$ and $\varepsilon^{\prime \prime}$ can be obtained based on the above mentioned assumptions for the terahertz time-domain spectroscopy. This general method, which applies to all practical cases, is not only highly reliable but also very fast, allowing a realtime extraction.

Figure 1.8 shows $\varepsilon$ and $\tan \delta$ variations. It is evident that the foam material has a refractive index close to 1 which is the refractive index of air. The increasing trend of the absorption factor in the high frequency region is evident; $\tan \delta \sim 0.001$ at $1 \mathrm{THz}$.

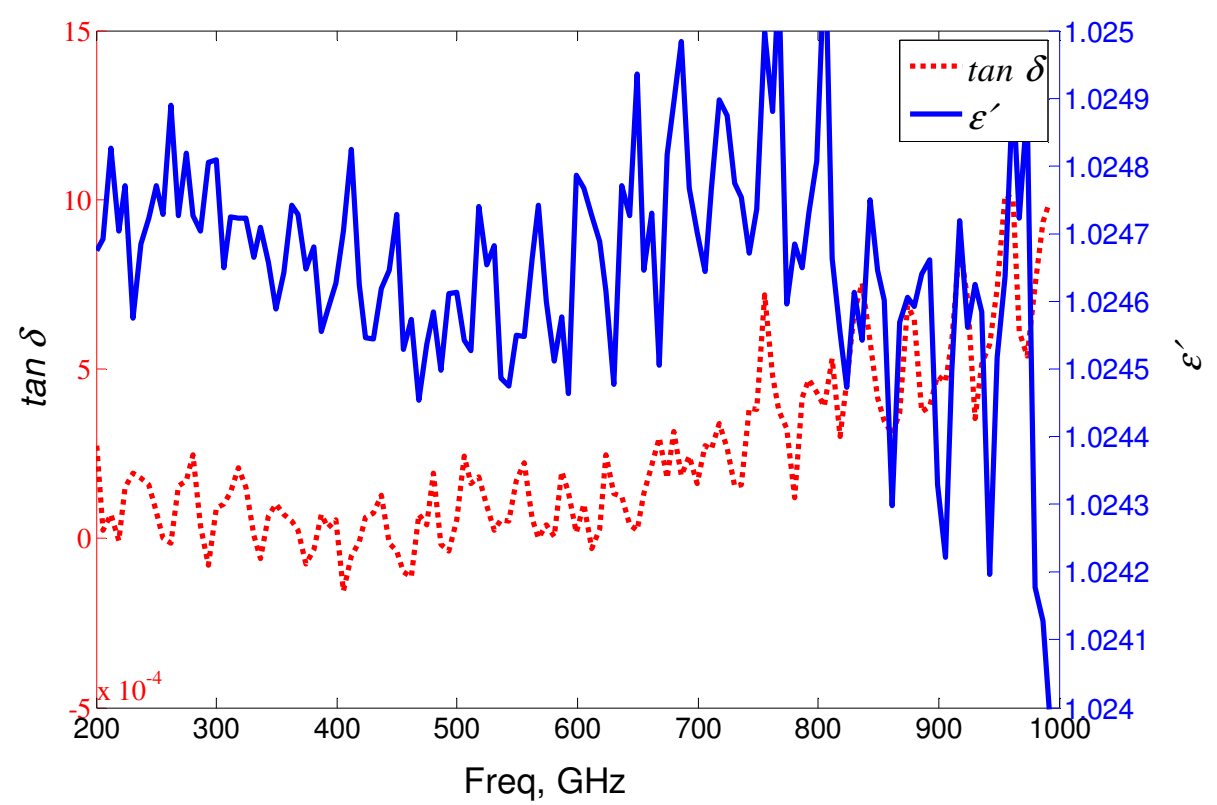

Figure $1.8 \varepsilon^{\prime}$ (the real part of permittivity index) is plotted with right ordinate and $\tan \delta$ (the loss tangent) is plotted with left ordinate both as functions of signal frequency [21]. 


\subsubsection{THz Finite Element Analysis}

Finite element analysis has been carried out using the COMSOL Multiphysics software. In the frequency domain for simulating the T-ray propagation in a semi-infinite air medium it is mandatory to eliminate the reflection at the artificial boundaries. Absorbing boundaries are used to prevent reflections. The material behaves dispersive and the dielectric properties are frequency dependent. The experimentally derived dielectric dispersion curves as shown in Figure 1.8 are given as input for the FE analysis. In order to achieve a smooth and uniform beam a Gaussian beam profile ([1]) is used with the beam waist $\left(w_{0}\right.$ ) equal to $10 \mathrm{~mm}$. The TDS machine configuration is such that the focused beam passing through the sample has frequency-independent waist size. The Gaussian beam profile on the left side of the domain boundary is defined as (see [22]):

$$
E(y)=E_{0} \exp \left(\frac{-y^{2}}{w_{0}{ }^{2}}\right)
$$

In which $E_{0}$ is the beam amplitude which is considered a unit value.

The governing equation to solve the TEM (transverse electro-magnetic) wave propagation problem is the Helmholtz equation with the vertically polarized Electric field as follows [23]:

$$
\begin{gathered}
\nabla \times\left(\mu_{\mathrm{r}}^{-1} \nabla \times \mathbf{E}\right)-\left(\varepsilon_{\mathbf{r}}-\frac{i \sigma}{\omega \varepsilon_{0}}\right) k_{0}{ }^{2} \mathbf{E}=0 \\
\mathbf{E}=E_{z} \mathbf{e}_{\mathbf{z}}
\end{gathered}
$$


In which $\sigma=$ electric conductivity (zero in our case), $\boldsymbol{E}_{\mathbf{r}}=$ relative permittivity (obtained from Figure 1.8 ), $k_{0}=$ free-space wave number and $\varepsilon_{0}=$ permittivity of free space.

The COMSOL generated result for the bounded $\mathrm{THz}$ beam propagating through the foam tile is shown in Figure 1.9. As shown in Figure 1.9 the Gaussian beam propagates from the left boundary of the problem and passes through the foam sample. The circle shows the hole position for the defective tile which is analyzed later. While generating Figure 1.9 the material inside the circle is assigned the same dielectric properties as the foam material to model a solid sample in absence of any defect.

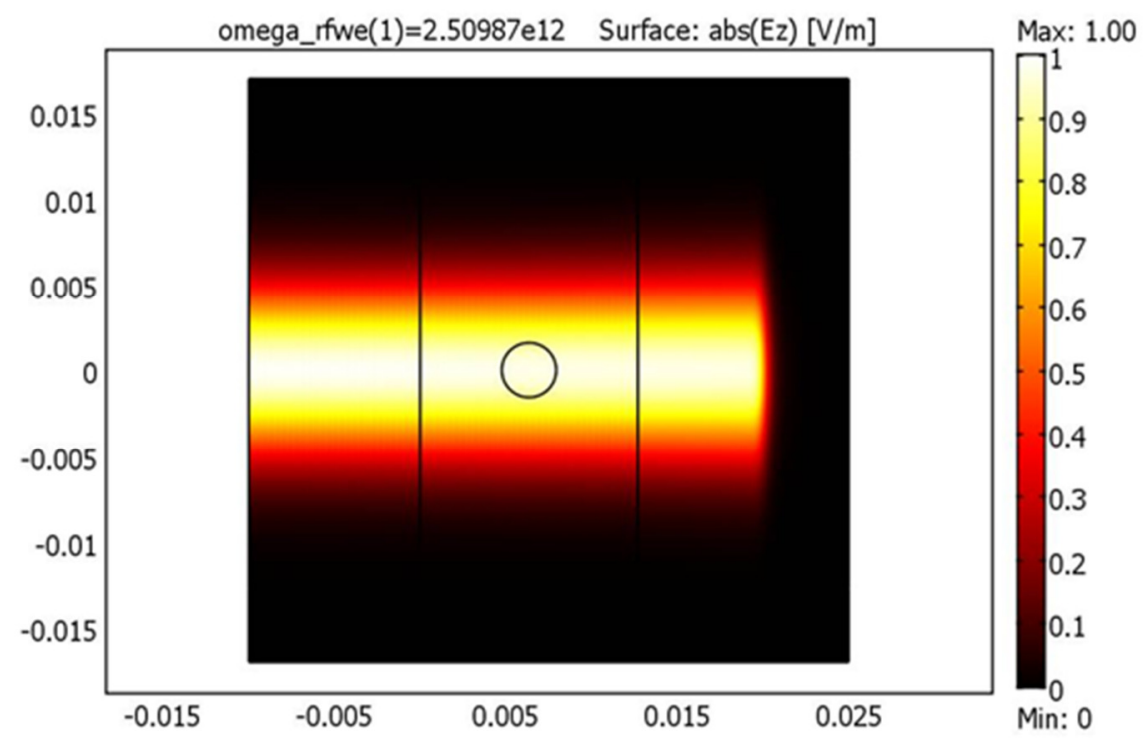

Figure 1.9 $\mathrm{THz}$ Gaussian beam (Freq=400 GHz) passing through a solid foam tile. The beam is propagating in the $x$-direction from left to right, penetrating through the foam tile placed between the two vertical lines. $e_{z}=$ unit vector normal to the 2D FE model [21].

Figure 1.10 shows the PT variation with frequency when the electric field through air is considered as the reference signal and the electric field through the solid foam is the 
sample signal, see equation (1.84). PT is close to $0 \mathrm{~dB}$ at lower frequencies which verifies the non-dispersive behavior in this frequency range. The dispersion increases at higher frequencies and it causes the deviation of PT from $0 \mathrm{~dB}$. Figure 1.11 shows a comparison between the FEM (finite element method) predictions obtained using the dispersive material properties calculated experimentally and the experimental PT values shown in equation (1.84) for the received signal strength after it is transmitted through the foam block. Good matching between the numerical and experimental results verifies the FEM modeling. After developing the reliable FE model the mechanical damage inside the specimen is introduced and changes in response are investigated.

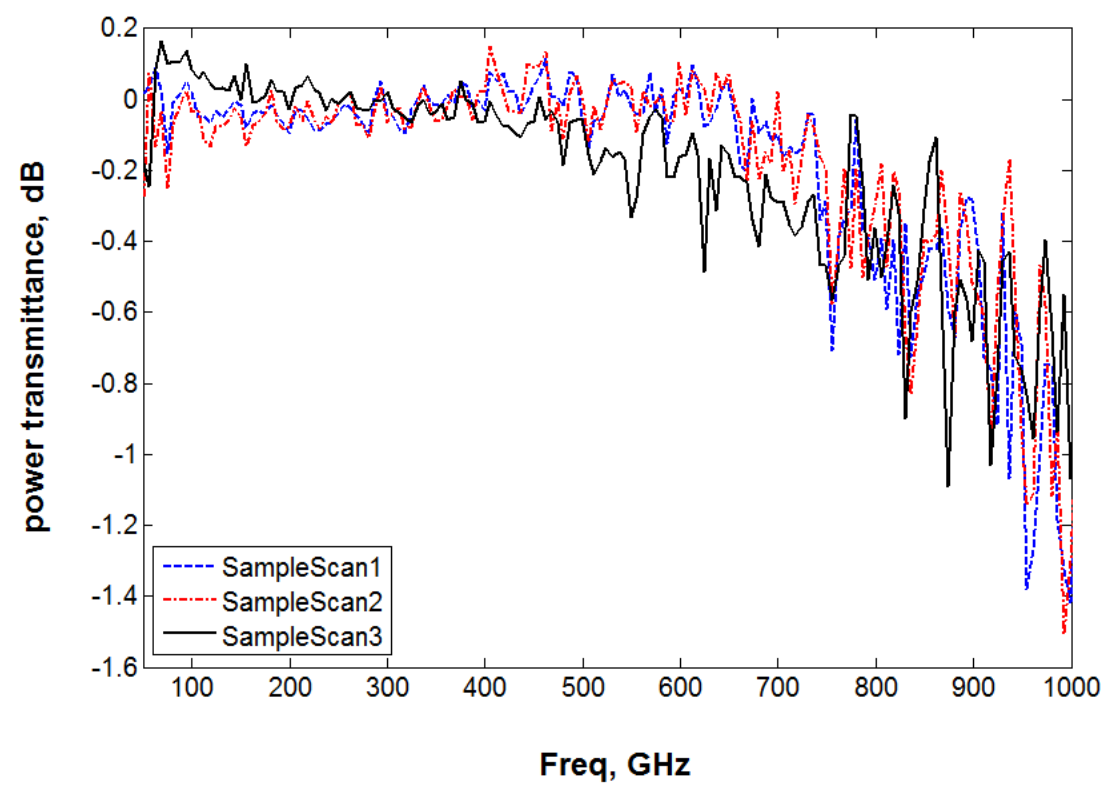

Figure 1.10 PT value for Reference = air, Sample = solid foam, see equation (1.84) [21]. 


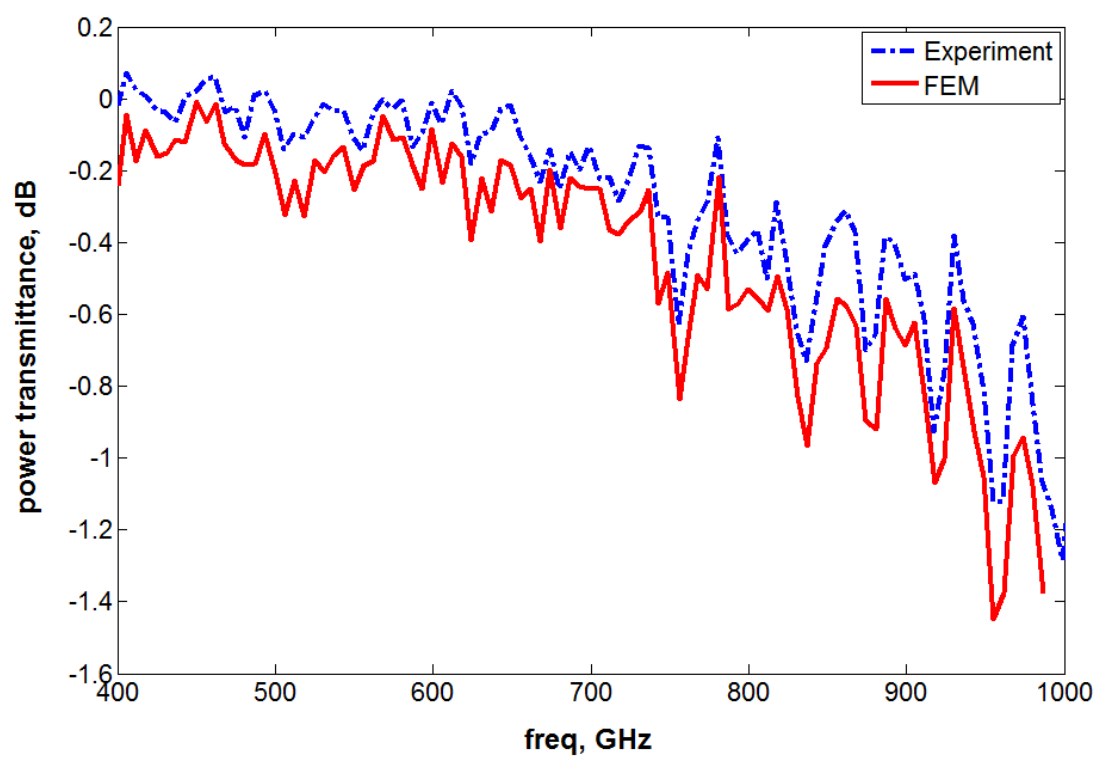

Figure 1.11 Comparison between experimental and FEM predicted PT values, Reference $=$ air, Sample $=$ solid foam, see equation (1.84) [21].

\subsubsection{THz-TDs measurement of blocks with mechanical damage}

Figure 1.12 compares the frequency-domain response of the received pulse passing through the non-defective sample (reference signal) and the damaged sample fabricated by drilling one-eighth inch $(\sim 3 \mathrm{~mm})$ diameter hole through the one inch wide foam. Figure 1.12 is generated by propagating the signal perpendicular to the hole axis. For the frequency range 100 to $400 \mathrm{GHz}$ the defect-free and the defective sample responses coincide, but for frequencies higher than $400 \mathrm{GHz}$ the damaged sample response lies below the reference response. This is because of diffraction occurring at the hole boundary in this frequency range. See the numerical simulation at $830 \mathrm{GHz}$ presented in Figure 1.13. The Gaussian beam strength variation across the beam cross section is assumed (see equation (1.85)). Diffraction of the beam hitting the circular interface decreases the field intensity behind the hole. 


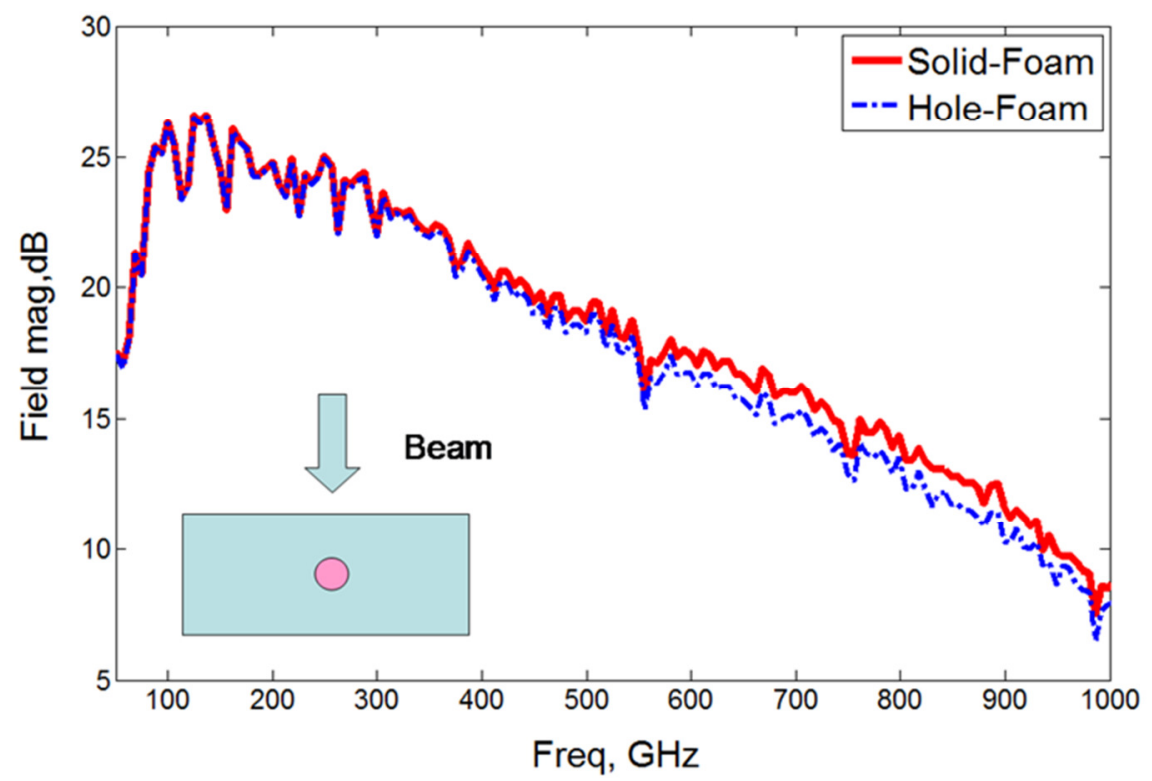

Figure 1.12 Frequency domain response for T-ray going through a defect-free foam tile and a foam tile with 1/8 inch $(\sim 3 \mathrm{~mm})$ diameter through-hole, as shown in the figure [21].

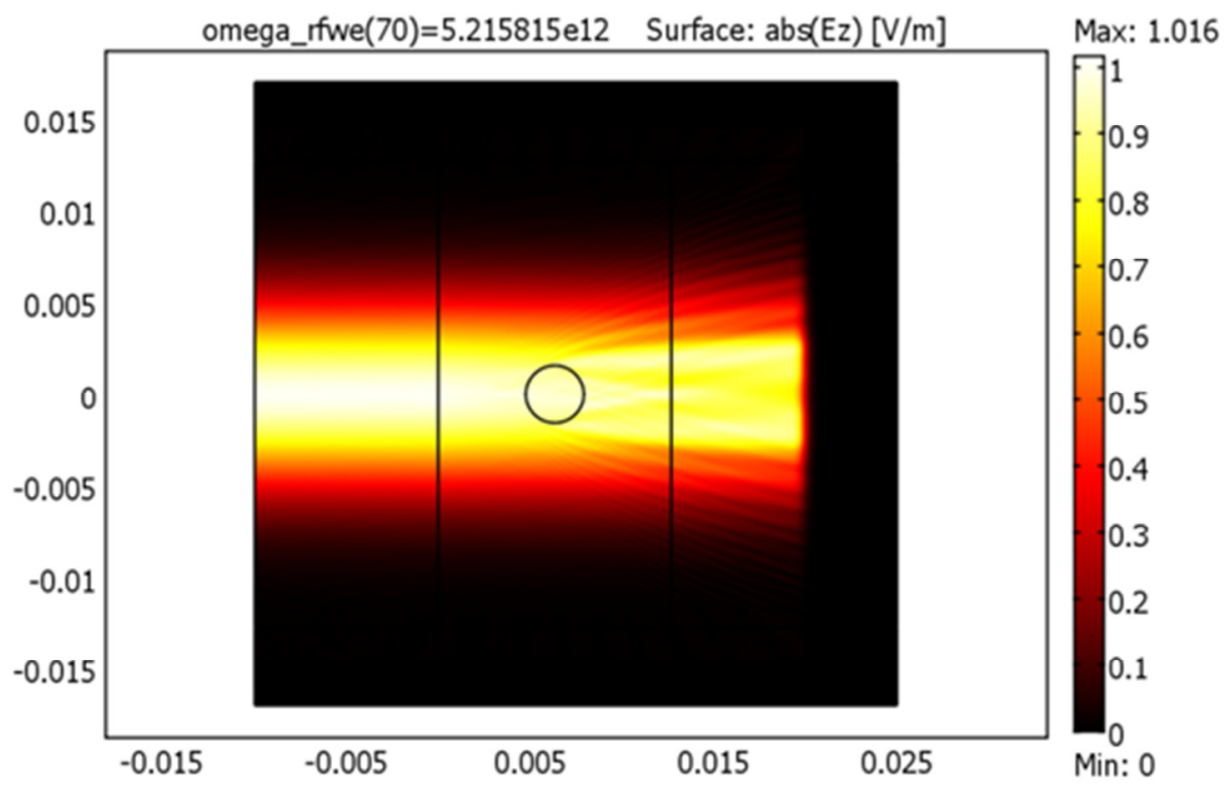

Figure 1.13 THz beam (Freq=830 GHz) passing through a foam tile with a hole, see Figure 1.12 [21]. 


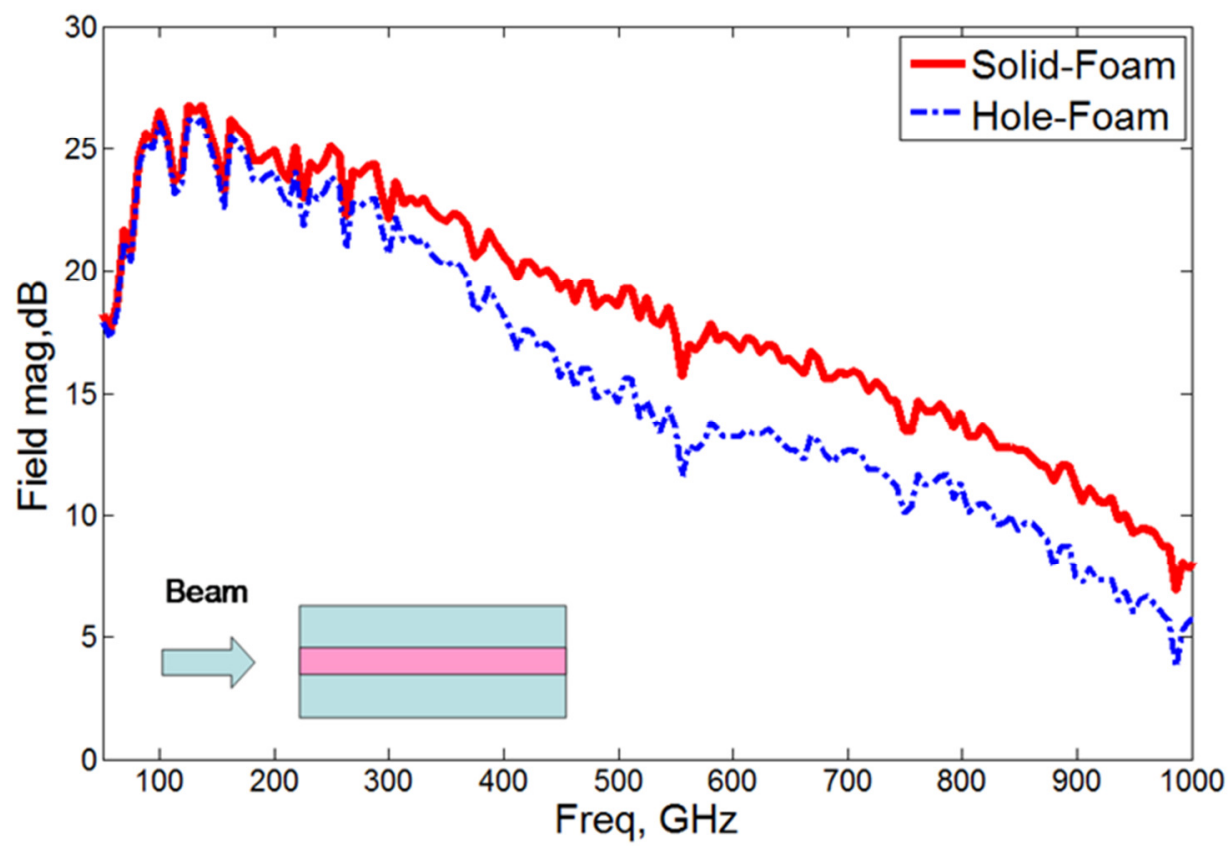

Figure 1.14 Frequency domain response for a defect-free foam tile and a foam tile with a 1/8 inch $(\sim 3 \mathrm{~mm})$ diameter hole. Beam propagates parallel to the hole axis [21].

Figure 1.14 shows a comparison between the frequency-domain response of the received pulse passing through the defect-free sample and the corresponding response when the beam passes through the hole propagating parallel to the hole axis. It is evident that for the frequency range 100 to $200 \mathrm{GHz}$ the defect-free and defective sample responses coincide. However, at frequencies higher than $200 \mathrm{GHz}$ the defective sample response lies below the response for the solid foam. It is the result of the diffraction occurring around the hole in this frequency range.

Figure 1.15 shows the PT value considering the signal passing through the solidfoam as the reference signal, and the signal passing through the defective foam as the sample signal. PT is around $0 \mathrm{~dB}$ at lower frequencies and a clear dip is observed at 550 GHz. Non-monotonic nature of Figure 1.15 is interesting and requires some explanation. 
In order to understand why the received signal strength should show a dip at $550 \mathrm{GHz}$ FEM simulation is carried out at three frequencies - 100, 550 and $1000 \mathrm{GHz}$. Figure 1.16a shows the strength of the Gaussian beam (the absolute value of the complex electric field in frequency domain) hitting the defective foam tile and passing along the hole axis at $100 \mathrm{GHz}$ frequency. Figure $1.16 \mathrm{~b}$ shows the wave fronts of the plotted field (the real part of the complex electric field in frequency domain). At $100 \mathrm{GHz}$ frequency because the wave length is large the signal passes through the defective sample almost without sensing the hole, as one can see in Figure 1.16b.

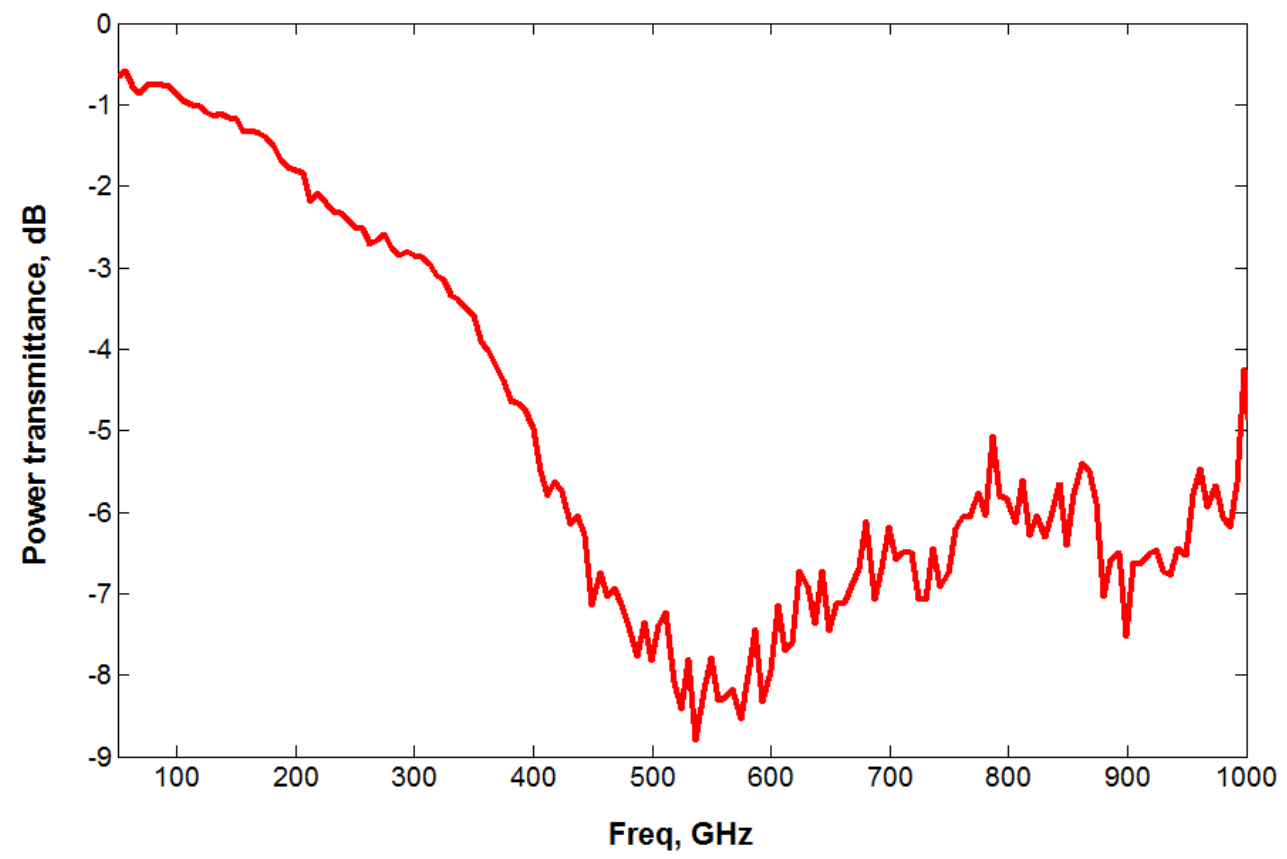

Figure 1.15 PT value for reference signal through the solid foam and sample signal through the defective foam, beam propagates parallel to the hole axis [21].

Since the diffraction of the beam is negligible at $100 \mathrm{GHz}$ the PT value is only -1 $\mathrm{dB}$ at this frequency. Figure 1.16c and Figure $1.16 \mathrm{~d}$ show similar plots for $550 \mathrm{GHz}$ signal frequency. In energy (Figure 1.16c) and wave front (Figure 1.16d) plots one can 
see that the incident beam is divided into two diverging beams, with a very weak beam in the middle. Therefore, at $550 \mathrm{GHz}$ the transmitted signal strength for the defective tile must be much smaller than that for the defect-free tile. It justifies a PT value of $-9 \mathrm{~dB}$ in Figure 1.15.

Figure 1.16e and Figure 1.16f show similar plots for $1000 \mathrm{GHz}$ signal frequency. In Figure 1.16e and Figure 1.16f one can see that the incident beam is divided into three beams. Because of the small wave length at $1 \mathrm{THz}$ frequency part of the energy can propagate through the air in the hole while the rest of the energy is scattered by the hole

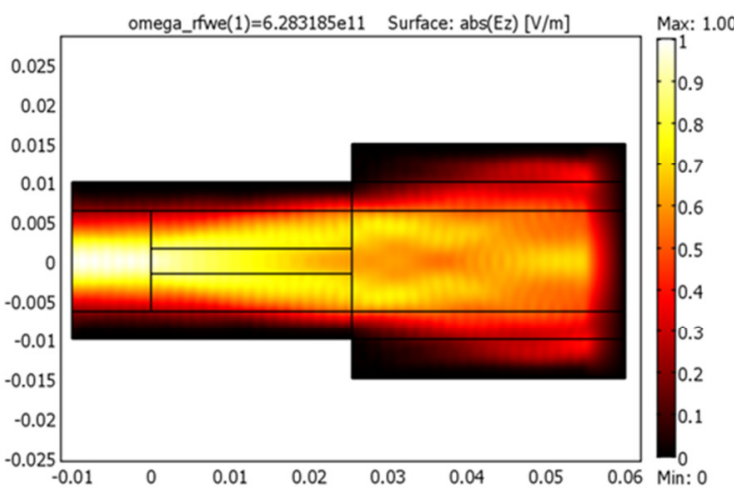

(a)

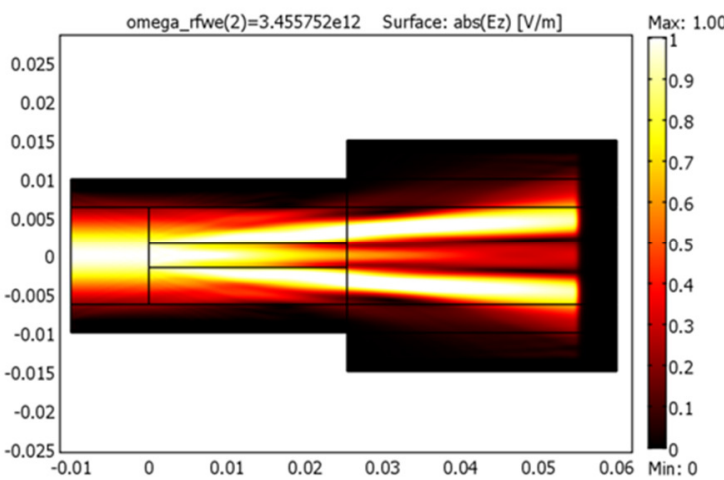

(c)

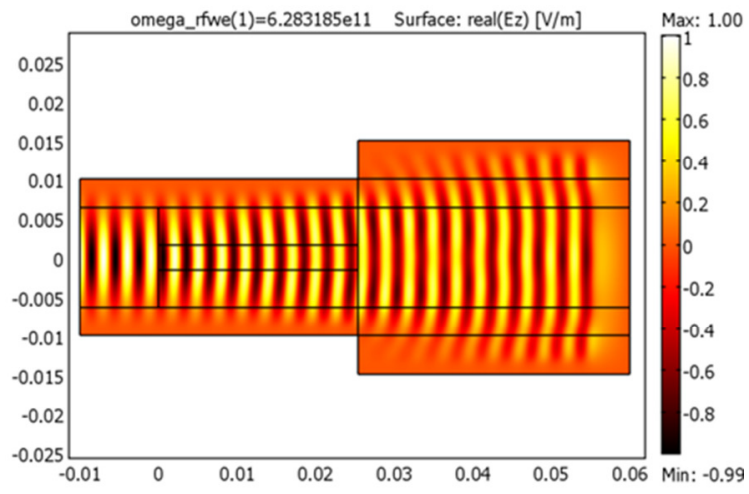

(b)

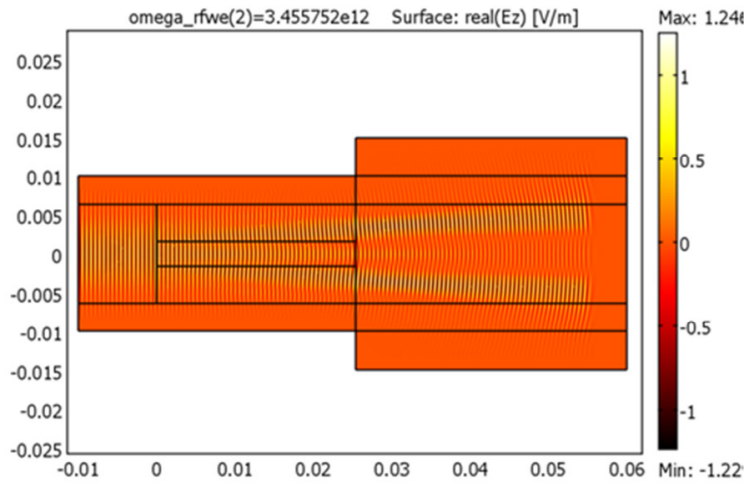

(d) 


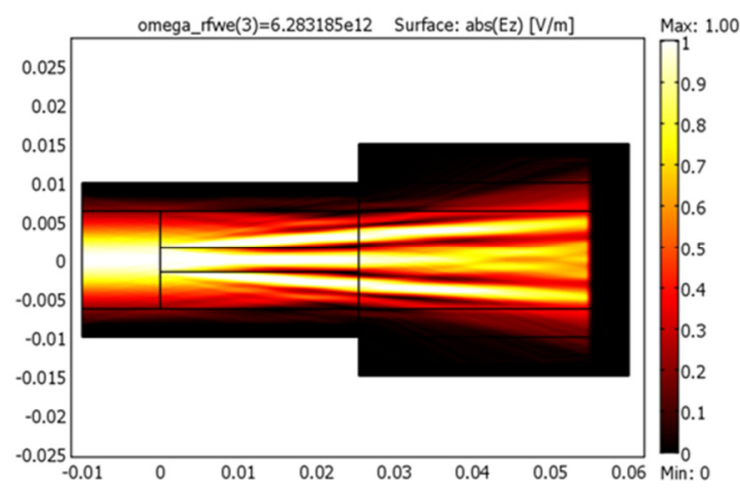

(e)

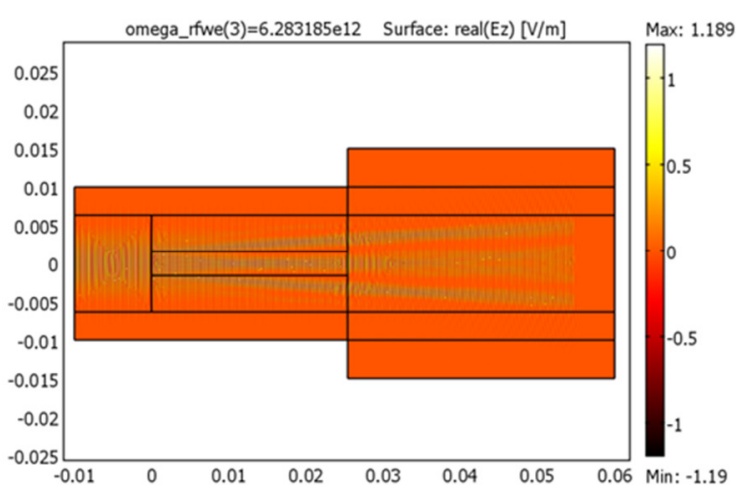

(f)

Figure 1.16 THz beam scattered by a cylindrical hole while propagating parallel to the central axis of the hole (a) energy distribution, freq = $100 \mathrm{GHz}$; (b) wave fronts, freq = $100 \mathrm{GHz}$; (c) energy distribution, freq = 550 GHz; (d) wave fronts, freq = $550 \mathrm{GHz}$; (e) energy distribution, freq = $1000 \mathrm{GHz}$; (f) wave front, freq $=1000 \mathrm{GHz}$ [21].

creating three beams in Figure 1.16e and Figure 1.16f. Clearly, the transmitted signal strength at the central axis at $1 \mathrm{THz}$ frequency is higher than that at $550 \mathrm{GHz}$ since less energy is diffracted away from the central axis in comparison to that at $550 \mathrm{GHz}$.

What is interesting to note here is that a cylindrical hole containing air in the polymer foam tile can be detected by $\mathrm{THz}$ radiation although the dielectric properties of air and the polymer are very close, as evident from Figure 1.8.

The cylindrical interface between these two very similar dielectric materials (polymer and air) can significantly scatter the $\mathrm{THz}$ beam in the frequency range 0.4 to $1 \mathrm{THz}$, as evident in Figure 1.12 to Figure 1.16. Therefore, inclusions having material properties close to that of the surrounding medium can be detected by $\mathrm{THz}$ beam due to the scattering of T-ray at the interface of the two materials. For detecting smaller than $1 / 8$ in diameter holes $\mathrm{THz}$ pulses with frequency content greater than $1 \mathrm{THz}$ is required. 


\subsection{Heat Induced Damage Detection by THz Radiation}

Use of TDS-THz to detect mechanical damage inside polymer foam material was investigated in section 1.5, both experimentally and numerically. The focus of this section is nondestructive evaluation of heat treated polymer tiles using pulsed THz-TDS to investigate if T-Ray is sensitive to heat damage. Extremely porous artificial pumice stone blocks made of polymers are subjected to heat exposures at temperatures ranging from room temperature to close to the melting point of the material. This kind of polymer showed some heat induced damage without melting in the oven. The dielectric properties of heat damaged samples are extracted and compared with those of the untreated samples to investigate if there exists any trend in material property change with the degree of heat damage. Since the specimens are heated for a long time the heat treatment is assumed to alter the sample properties uniformly.

\subsubsection{Specimen Preparation}

As shown in Figure $1.17 \mathrm{a}$ artificial pumice stone pieces were cut into approximately $5 \times 3.5 \times 2.5 \mathrm{~cm}$ blocks and were exposed to thermal loads. Five almost identical blocks B1 to B5 were chosen for heat treatment. Although the blocks were cut under identical conditions small variations in thickness were noted after they were fabricated and heat treated. Measured thicknesses of Blocks B1 to B5 after heat treatment were $25.33,24.79,25.49,25.05$ and $24.05 \mathrm{~mm}$, respectively. B1 was subjected to no heat treatment (was kept at room temperature) while the other four blocks were heated at 200, 400, 600 and 800 degree centigrade, respectively for 24 hours (see Figure 1.17b). The long exposure to the high temperature was intended to make sure that the heat penetrates 
into the core and uniformly increases the temperature of the specimen. The melting point of the block material was close to $900^{\circ} \mathrm{C}$. The specimens were visually inspected before and after the heat exposure and small change in the surface color from sky-blue to grayblue was noted but no macro mechanical damage or crack was observed. Since the pumice stones as shown in Figure 1.17a are porous the ultrasonic signals cannot penetrate through the specimen. As a result, one has to depend on the $\mathrm{THz}$ technique to inspect heat induced damages in such highly porous specimens.

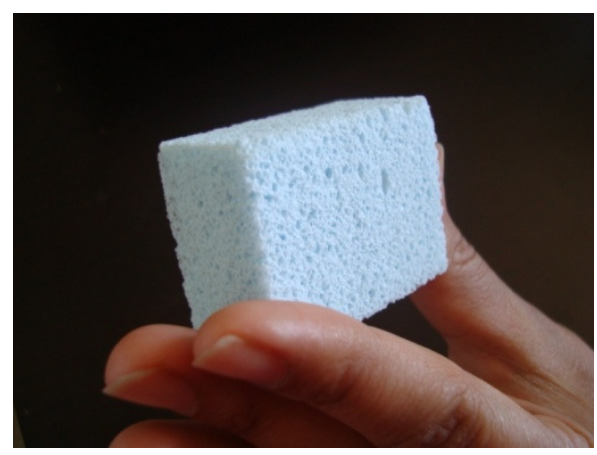

(a)

\begin{tabular}{|c|c|}
\hline T1 & Room Temp. \\
\hline T2 & $200^{\circ} \mathrm{C}$ \\
\hline T3 & $400^{\circ} \mathrm{C}$ \\
\hline T4 & $600^{\circ} \mathrm{C}$ \\
\hline T5 & $800^{\circ} \mathrm{C}$ \\
\hline
\end{tabular}

(b)

Figure 1.17 (a) Porous material specimen. (b) Heat treatment temperatures for Blocks B1 to B5 are denoted as T1 to T5, respectively [20].

\subsubsection{THz-TDs measurement of heat treated blocks}

Similar to the method presented in section 1.5 for dielectric material property determination, each specimen is placed on the path of the terahertz pulse beam between the emitter and the detector (see Figure 1.5). Each Block was scanned 6 times to minimize the possible experimental errors. Figure 1.18 compares the TDS generated reference signal through the free-space with the signals passing through blocks B1, B3 
and B5. In order to calculate the dielectric constants, the time domain electric field responses are converted to frequency-domain. As shown in Figure 1.19, in the frequencydomain response of the received pulse only a small difference exists between the signals passing through the air (the reference signal) and through the blocks (the sample signals) for frequencies below $150 \mathrm{GHz}(0.15 \mathrm{THz})$, while at higher frequencies a significant separation of signals are noticed. It should be noted that, in this investigation the frequency range of interest is from $120 \mathrm{GHz}$ to $240 \mathrm{GHz}$ which can still be considered Tray that covers from $0.1 \mathrm{THz}$ to about $5 \mathrm{THz}$.

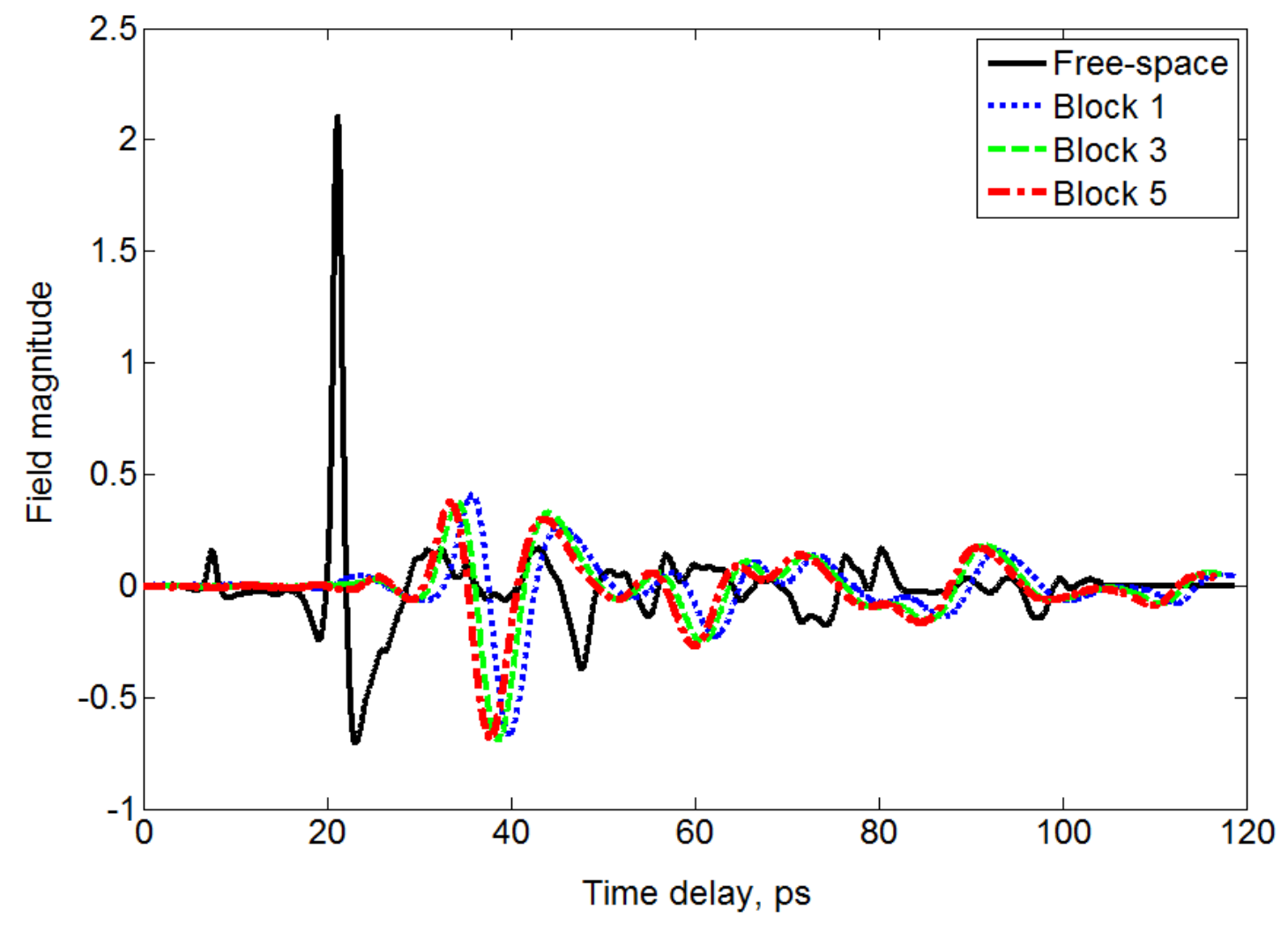

Figure 1.18 Received THz pulse propagated through free-space and Blocks 1, 3 and 5 [20]. 
Figure 1.19 shows $\varepsilon$ and $\tan \delta$ (see equations (1.73) to (1.75)) variations for blocks B1 to B5. It is evident that the block material has a refractive index close to 1 similar to that of air. The increasing trend of the absorption factor in the high frequency range is evident. A consistent trend (monotonic variation of absorption with temperature) was expected for the received signal from the five blocks.

However, in Figure 1.19, the curve corresponding to block B5 is found between the curves corresponding to B1 and B3. This is because of slight variations of thickness of samples B1, B3 and B5. When the thickness independent dielectric properties of the samples are evaluated by properly considering the small thickness variations a consistent trend is observed as shown in Figure 1.20.

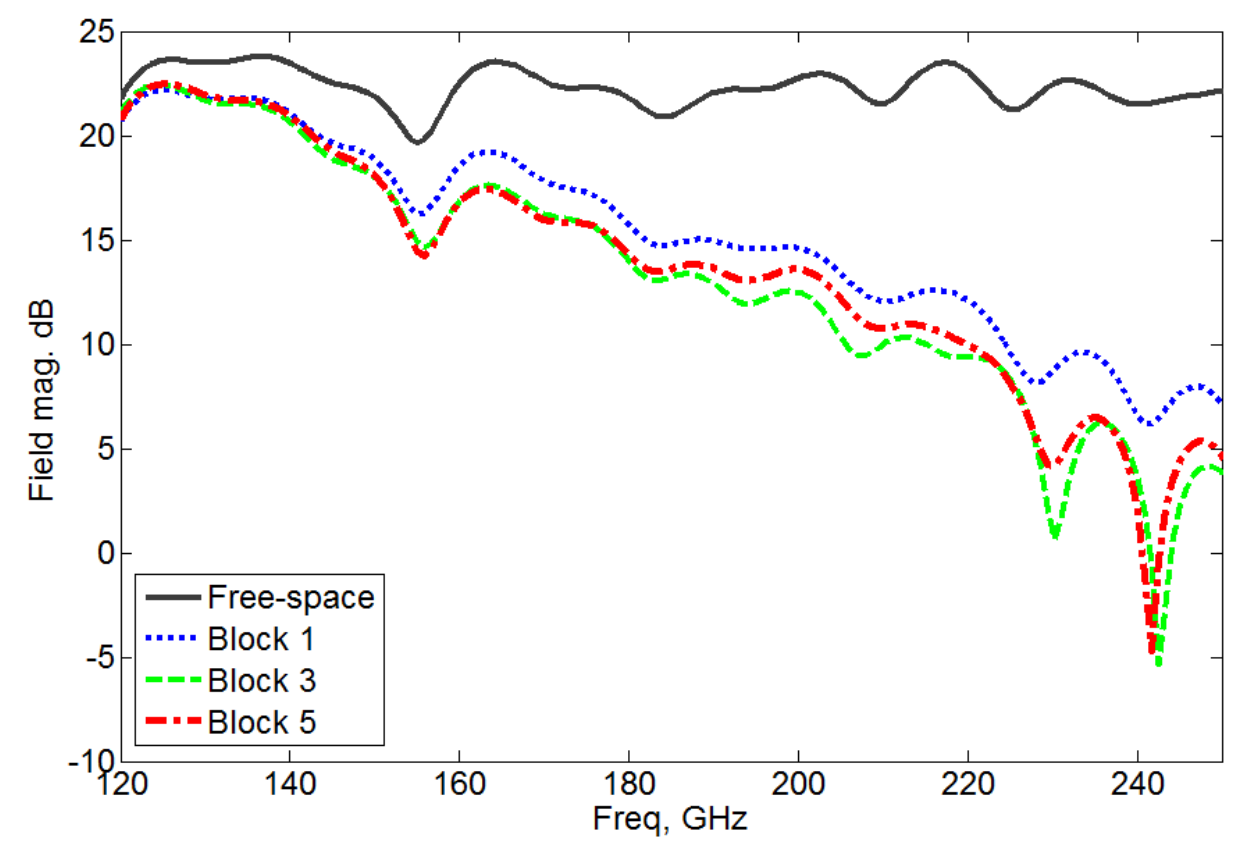

Figure 1.19 Frequency domain response for the free-space and Blocks 1, 3 and 5 [20]. 
Figure 1.20a shows $\varepsilon$ ' is decreasing with increasing heat exposure temperature. Note that the five curves fall into two groups separated by a noticeable gap - T1 and T2 are in one group and T3 to T5 are in another group. Figure 1.20b also shows a clear separation of the curves into two groups, one containing T1 and T2 and the other group is consisting of T3 to T5. Clearly, the block material undergoes a major change between $200^{\circ} \mathrm{C}$ and $400^{\circ} \mathrm{C}$ causing a noticeable variation in the material's dielectric properties.

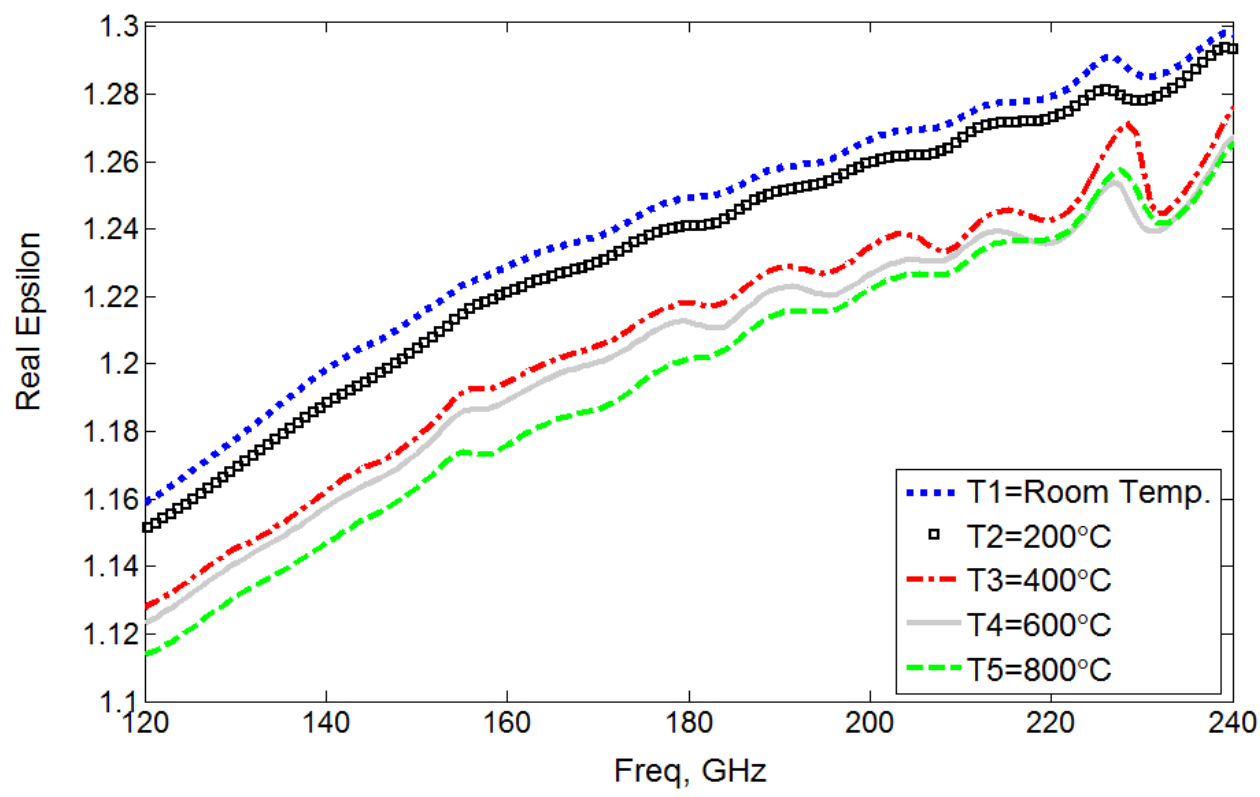

(a) 


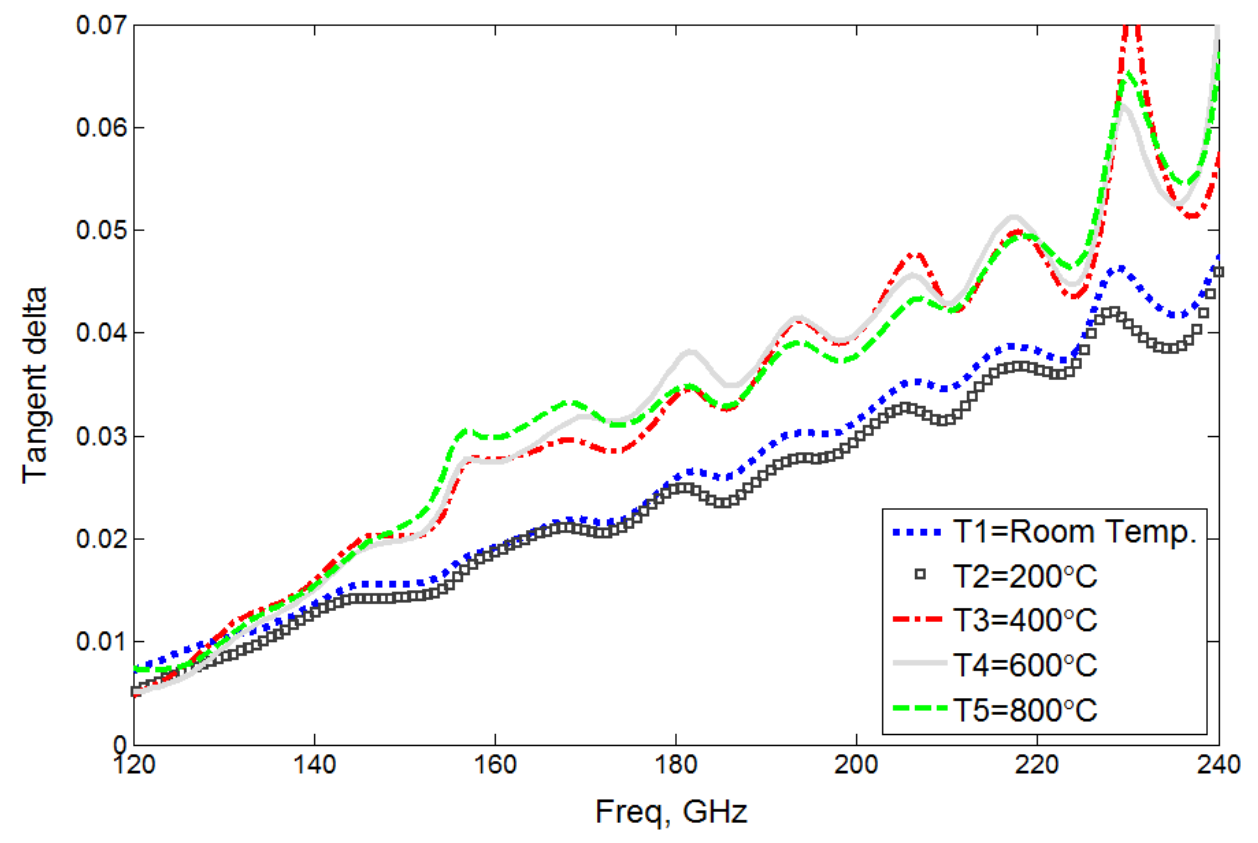

(b)

Figure 1.20 (a) $\varepsilon^{\prime}(\omega)$ (the real part of permittivity index) and (b) $\tan \delta$ (the loss tangent) variations as functions of signal frequency for the five samples [20].

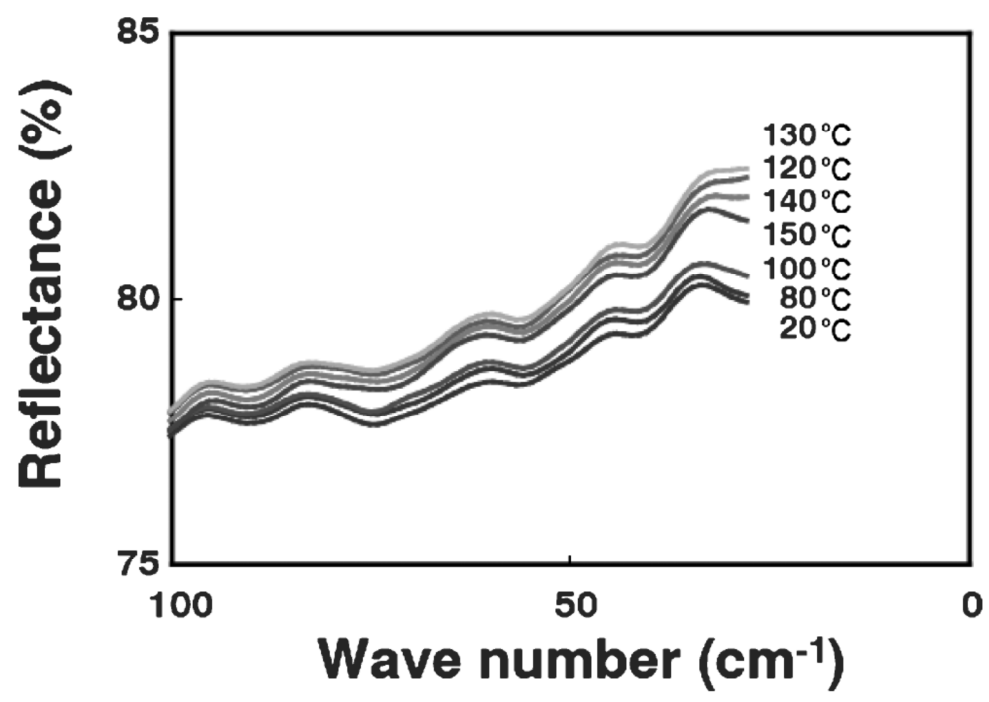

Figure 1.21 Temperature dependence of FIR reflection spectra below100 $\mathrm{cm}^{-1}$ wave number for the $\mathrm{BaTiO}_{3}$ ceramics (from Hoshina et al. [24]) 
It is important to note that the dielectric properties thus extracted are effective properties. Since the samples are porous, if it is necessary to compute the real dielectric properties of polymer itself without any porosity for different heat exposure levels, then one will need to measure the porosity and follow the effective medium theory (see [25]).

Hoshina et al. [24] evaluated the Temperature dependence of Far Infrared reflection (FIR) spectra below $100 \mathrm{~cm}^{-1}$ wave number for the $\mathrm{BaTiO}_{3}$ ceramics. Figure 1.21 shows the wave number vs. reflectance curves falling into two groups - one group contains curves corresponding to $100^{\circ} \mathrm{C}$ and lower temperatures and the second group contains curves above $100^{\circ} \mathrm{C}$. Clearly some significant change in material properties occurred between $100^{\circ} \mathrm{C}$ and $120^{\circ} \mathrm{C}$. Similarly, for a different material, as shown here, Rahani et al. [20] found a significant change in the material dielectric properties between $200^{\circ} \mathrm{C}$ and $400^{\circ} \mathrm{C}$, well below its melting point. One interesting similarity between the results in Figure 1.20 and Hoshina's results shown in Figure 1.21 is the observation of some non-monotonic response with the heat-treatment temperature variation. Also, in both results the curves fall into two main groups indicating some significant change in dielectric properties of the material well below its melting point.

\subsection{DPSM for THz radiation modeling}

From modeling point of view, the wide spread applications of the relatively young $\mathrm{THz}$ technology warrants new analytical, semi-analytical and numerical techniques. Distributed Point Source Method (DPSM) a semi-analytical technique for modeling ultrasonic wave propagation problems has gained popularity for modeling ultrasonic NDE (Nondestructive Evaluation) problems in recent years. The basic principle of DPSM 
has been described in Refs. [26-28]. These references compared FEM and DPSM techniques for modeling circular and rectangular transducers. It is shown there that FEM3D at high frequencies encounters the accuracy problem unless the domain is meshed with very small finite elements that make this method computationally very time consuming while the DPSM technique is much faster and produces results that match very well with the analytical solutions. Ultrasonic field modeling in multilayered structures using DPSM technique was reported by Banerjee et al. [29, 30]. Scattering of ultrasonic waves by spherical and elliptical cavities in solid half-space has been investigated in Refs.[31, 32].

Many numerical techniques for solving electrostatic and electromagnetic scattering problems involve Green's functions or point source solutions. Some of the popular methods in this category are Method of Auxiliary Sources (MAS), Method of Moments (MoM), Discrete Dipole Approximation (DDA), Discrete Singularity Method (DSM), Multiple Multipole Technique (MMT), Generalized Multipole Techniques (GMT), Current Model Method (CMM), Charge Simulation Method (CSM), etc. For a good review of these numerical techniques readers are referred to Refs. [33, 34]. Among these techniques the method which is closest to DPSM is MAS. In both these methods auxiliary point sources are placed away from the physical boundary and interface to avoid the difficulty associated with the singularity of the Green's function. Thus they completely eliminate the Green's function singularity problem of a typical MoM kernel. The implementation of DPSM and MAS is also conceptually simpler. Satisfying the boundary conditions at discrete set of collocation points a matrix equation is derived 
instead of an integral equation, avoiding the necessity of any MoM transformations. Furthermore, since each solution in the set is analytically known, there is no need to integrate currents in order to determine fields at any stage of the solution [34].

One disadvantage of MoM and DDA is the need to repeat calculations for each new angle of incidence of the external field. Long-wavelength approximation is applied in the formulation of both MoM and DDA [33]; as a result small volume elements must be implemented for accurate results. Therefore, meshing a large domain with small volume elements makes these methods computationally expensive.

Other widely accepted numerical methods such as Finite Difference Method (see $[35,36])$ can be also computationally very expensive at high frequencies and for large domains because it requires very fine mesh of the whole domain. Furthermore the absorbing boundaries should be taken care of carefully to avoid unwanted boundary reflections. The Green's function based methods like DPSM and MAS do not need to define any absorbing boundary and increasing the wave propagation domain size does not affect the computation cost unless the boundary or interface size changes.

Although the fundamental principles of DPSM and MAS are the same they differ in their detailed implementation. For a simple wave scattering problem involving a scatterer in an infinite medium subjected to an incident electric field, these two techniques are identical. MAS has been mostly applied to solve such problems. However, for solving waveguide or multi-layered dielectric problems eigenmodes satisfying the boundary and interface conditions are used as the basis functions or Green's functions in MAS [37]. As a result basis functions become dependent on the 
problem geometry. It also restricts what geometries can be handled by MAS, since eigen modes for all geometries are not available. DPSM on the other hand takes Green's functions of infinite space as the basis functions for any structure and thus independent of the problem geometry [29-32] . Although, in principle MAS can be developed with fullspace Green's functions for any geometry it has not been developed yet for multi-layered structures [37]. The DPSM method is used here to model electromagnetic wave propagation problems in $\mathrm{THz}$ frequency range.

\subsubsection{Gaussian beam theory}

In optics, a Gaussian beam is defined as a beam of electromagnetic radiation whose transverse electric field and intensity (irradiance) distributions are described by the Gaussian function. Many lasers emit beams with a Gaussian profile; the laser is said to be operating on the fundamental transverse mode, or "TEM00 mode" of the laser's optical resonator.

Equation (1.88) that describes the Gaussian beam is a solution of the Helmholtz equation in the paraxial form. The solution, in the form of a Gaussian function, represents the complex amplitude of the electric field, which propagates along with the corresponding magnetic field as an electromagnetic wave in the beam [38, 39].

$$
\begin{gathered}
E(r, z)=E_{0} \frac{w_{0}}{w(z)} \exp \left(\frac{-r^{2}}{w^{2}(z)}\right) \exp \left(-i k z-i k \frac{r^{2}}{2 R(z)}+i \zeta(z)\right) \\
w(z)=w_{0} \sqrt{1+\left(\frac{z}{z_{R}}\right)^{2}}
\end{gathered}
$$




$$
\begin{gathered}
z_{R}=\frac{\pi w_{0}^{2}}{\lambda} \\
R(z)=z\left[1+\left(\frac{z_{R}}{z}\right)^{2}\right] \\
\zeta(z)=\tan ^{-1}\left(\frac{z}{z_{R}}\right)
\end{gathered}
$$

In which $k=2 \pi / \lambda$ is the wave number, $w_{0}$ is the beam waist, $z_{R}$ is Rayleigh range, $R(z)$ is radius of curvature and $\zeta(z)$ is the longitudinal phase delay or Gouy phase of the beam.

\subsubsection{Green's Functions \& DPSM}

The Electric and Magnetic fields in terms of Vector and Scalar potentials (A and V) are given by ([23]):

$$
\begin{gathered}
\mathbf{B}=\nabla \times \mathbf{A} \\
\mathbf{E}=-\nabla V-\frac{\partial \mathbf{A}}{\partial t}
\end{gathered}
$$

From equations (1.4), (1.10) and (1.13):

$$
\nabla \times(\nabla \times \mathbf{A})=\mu \mathbf{J}_{f}+\mu \varepsilon \frac{\partial\left(-\nabla V-\frac{\partial \mathbf{A}}{\partial t}\right)}{\partial t}
$$




$$
\nabla^{2} \mathbf{A}-\mu \epsilon \frac{\partial^{2} \mathbf{A}}{\partial t^{2}}=-\mu \mathbf{J}_{f}+\nabla\left(\mu \varepsilon \frac{\partial V}{\partial t}+\nabla . \mathbf{A}\right)
$$

In which:

$\varepsilon=$ Permittivity of the Linear Medium, $\mathrm{C}^{2} / \mathrm{N} \cdot \mathrm{m}^{2}$

$\mu=$ Permeability of the Linear Medium, N/A ${ }^{2}$

After separating the Eddy Current ( $\sigma \mathbf{E}$ ) and Currents due to free sources $\mathbf{J}_{f, s}$

$$
\begin{gathered}
\mathbf{J}_{f}=\sigma \mathbf{E}+\mathbf{J}_{f, s}=\sigma\left(-\nabla V-\frac{\partial \mathbf{A}}{\partial t}\right)+\mathbf{J}_{f, s} \\
\nabla^{2} \mathbf{A}-\mu \varepsilon \frac{\partial^{2} \mathbf{A}}{\partial t^{2}}=-\mu\left(\sigma\left(-\nabla V-\frac{\partial \mathbf{A}}{\partial t}\right)+\mathbf{J}_{f, s}\right)+\nabla\left(\mu \varepsilon \frac{\partial V}{\partial t}+\nabla \cdot \mathbf{A}\right)
\end{gathered}
$$

And considering Lorenz Gauge gives:

$$
\begin{gathered}
\nabla^{2} \mathbf{A}-\mu \varepsilon \frac{\partial^{2} \mathbf{A}}{\partial t^{2}}-\mu \sigma \frac{\partial \mathbf{A}}{\partial t}=-\mu \mathbf{J}_{f, s} \\
\mu \varepsilon \frac{\partial V}{\partial t}+\mu \sigma V+\nabla \cdot \mathbf{A}=0
\end{gathered}
$$

Assuming time harmonic response, equations (1.94) and (1.100) give:

$$
\nabla V=\frac{\nabla(\nabla \cdot \mathbf{A})}{(i \omega \mu \varepsilon-\mu \sigma)}
$$




$$
\mathbf{E}=-\nabla V+i \omega \mathbf{A}=\frac{\nabla(\nabla . \mathbf{A})}{(\mu \sigma-i \omega \mu \varepsilon)}+i \omega \mathbf{A}
$$

We can rewrite equation (1.100) in the form:

$$
\begin{aligned}
& \nabla^{2} \mathbf{A}+k^{2} \mathbf{A}=-\mu \mathbf{J}_{f, s} \\
& k^{2}=\mu \varepsilon \omega^{2}+i \mu \sigma \omega
\end{aligned}
$$

Considering the potential equation due to a Dirac pulse excitation at source point $\boldsymbol{x}_{s}$ :

$$
\begin{gathered}
\nabla^{2} \mathbf{A}+k^{2} \mathbf{A}=4 \pi \delta\left(\boldsymbol{x}_{s}\right) \\
A_{j}=G=\frac{\exp (-i k r)}{r}
\end{gathered}
$$

$\mathbf{A}=\left(A_{1}, A_{2}, A_{3}\right)$ is the Green's function vector and $r$ is the distance of target point $\boldsymbol{x}_{t}$ from the source point $\boldsymbol{x}_{s}$.

In DPSM discrete point sources are distributed near boundaries and interfaces. It is assumed that each point source has three components of strength $J_{1}, J_{2}, J_{3}$ that are computed in such a way that the boundary conditions and the compatibility conditions at interfaces are satisfied. The potential field vector due to a single point source using equation (1.106), in terms of $J_{1}, J_{2}, J_{3}$ and Green's function $G$ is defined as:

$$
\mathbf{A}=[\mathbf{P A}] \mathbf{J}
$$

In which:

$$
\mathbf{J}=\left\{\begin{array}{lll}
J_{1} & J_{2} & J_{3}
\end{array}\right\}^{\mathrm{T}} \quad, \quad \mathbf{P A}=G\left[\begin{array}{lll}
1 & 0 & 0 \\
0 & 1 & 0 \\
0 & 0 & 1
\end{array}\right]
$$


We define the first and second derivatives of $r$ with respect to three coordinate components as:

$$
\begin{gathered}
r d_{m}=\frac{\partial r}{\partial x_{m}}=\frac{\left(x_{m}-x_{m s}\right)}{r} \\
r d_{m n}=\frac{\partial^{2} r}{\partial x_{m} \partial x_{n}}=\frac{\delta_{m n}}{r}-\frac{\left(x_{m}-x_{m s}\right)}{r^{2}} \frac{\partial r}{\partial x_{n}}=\frac{\delta_{m n}-r d_{m} r d_{n}}{r}
\end{gathered}
$$

In which $\boldsymbol{x}_{m}$ and $\boldsymbol{x}_{m s}$ for $m=1,2,3$ are the target and source points, respectively. Also the first and second derivatives of the Green's function are derived as:

$$
\begin{gathered}
G d_{m}=\frac{\partial G}{\partial x_{m}}=-G\left(\frac{1}{r}+i k\right) \frac{\partial r}{\partial x_{m}}=-G\left(\frac{1}{r}+i k\right) r d_{m} \\
G d_{m n}=\frac{\partial^{2} G}{\partial x_{m} \partial x_{n}}=-\frac{\partial G}{\partial x_{n}}\left(\frac{1}{r}+i k\right) r d_{m}-G\left(\frac{1}{r}+i k\right) r d_{m n} \\
+G\left(\frac{1}{r^{2}}\right) r d_{m} r d_{n}=G\left[\left(\frac{1}{r}+i k\right)^{2} r d_{m} r d_{n}-\left(\frac{1}{r}+i k\right) r d_{m n}+\left(\frac{1}{r^{2}}\right) r d_{m} r d_{n}\right] \\
\nabla(\nabla . \mathbf{A}(\mathbf{r}))=\left[\begin{array}{l}
A_{1,11}+A_{2,21}+A_{3,31} \\
A_{1,12}+A_{2,22}+A_{3,32} \\
A_{1,13}+A_{2,23}+A_{3,33}
\end{array}\right] \\
A_{m, n p}=\frac{\partial^{2} A_{m}}{\partial x_{n} \partial x_{p}}=J_{m} \frac{\partial^{2} G}{\partial x_{n} \partial x_{p}}=J_{m} G d_{n p}
\end{gathered}
$$

The variation of potential vector due to a single point source along the unit vector $\mathbf{n}$ is given by: 


$$
\frac{\partial \mathbf{A}}{\partial \mathbf{n}}=\left[\begin{array}{lll}
\frac{\partial \mathrm{A}_{1}}{\partial \mathrm{x}_{1}} & \frac{\partial \mathrm{A}_{1}}{\partial \mathrm{x}_{2}} & \frac{\partial \mathrm{A}_{1}}{\partial \mathrm{x}_{3}} \\
\frac{\partial \mathrm{A}_{2}}{\partial \mathrm{x}_{1}} & \frac{\partial \mathrm{A}_{2}}{\partial \mathrm{x}_{2}} & \frac{\partial \mathrm{A}_{2}}{\partial \mathrm{x}_{3}} \\
\frac{\partial \mathrm{A}_{3}}{\partial \mathrm{x}_{1}} & \frac{\partial \mathrm{A}_{3}}{\partial \mathrm{x}_{2}} & \frac{\partial \mathrm{A}_{3}}{\partial \mathrm{x}_{3}}
\end{array}\right]\left\{\begin{array}{l}
\mathrm{n}_{1} \\
\mathrm{n}_{2} \\
\mathrm{n}_{3}
\end{array}\right\}=\left[\begin{array}{lll}
J_{1} G d_{1} & J_{1} G d_{2} & J_{1} G d_{3} \\
J_{2} G d_{1} & J_{2} G d_{2} & J_{2} G d_{3} \\
J_{3} G d_{1} & J_{3} G d_{2} & J_{3} G d_{3}
\end{array}\right]\left\{\begin{array}{l}
\mathrm{n}_{1} \\
\mathrm{n}_{2} \\
\mathrm{n}_{3}
\end{array}\right\}=[\mathbf{G A}] \mathbf{J}
$$

In which:

$$
\mathbf{G A}=\left(G d_{1} \mathrm{n}_{1}+G d_{2} \mathrm{n}_{2}+G d_{3} \mathrm{n}_{3}\right)\left[\begin{array}{ccc}
1 & 0 & 0 \\
0 & 1 & 0 \\
0 & 0 & 1
\end{array}\right]
$$

Finally, the Electric field vector in terms of Green's functions and three point source strength components $J_{1}, J_{2}, J_{3}$ is given as:

$$
E_{m}(\mathbf{r})=\frac{J_{1} G d_{1 m}(\mathbf{r})+J_{2} G d_{2 m}(\mathbf{r})+J_{3} G d_{3 m}(\mathbf{r})}{(\mu \sigma-i \omega \mu \varepsilon)}+i \omega J_{m} G(\mathbf{r})
$$

Rewriting equation (1.117) in matrix form gives:

$$
\mathbf{E}=[\mathbf{E M}] \mathbf{J}
$$

In which

$$
\mathbf{E M}=\frac{1}{(\mu \sigma-i \omega \mu \varepsilon)}\left[\begin{array}{lll}
G d_{11} & G d_{21} & G d_{31} \\
G d_{12} & G d_{22} & G d_{32} \\
G d_{13} & G d_{23} & G d_{33}
\end{array}\right]+\left[\begin{array}{ccc}
i \omega G & 0 & 0 \\
0 & i \omega G & 0 \\
0 & 0 & i \omega G
\end{array}\right]
$$




\subsubsection{Gaussian Beam modeling in DPSM}

A DPSM model is suggested for the Gaussian beam (see Figure 1.22). The theoretical unbounded beam has been truncated to form a bounded beam that has one set of sources; its strength vector is denoted as $\mathbf{X}_{\mathbf{s}(3 \mathrm{~N} \times 1)}=\left\{\begin{array}{lllll}J_{1} & J_{2} & \cdots & J_{N-1} & J_{N}\end{array}\right\}^{\mathrm{T}}$.

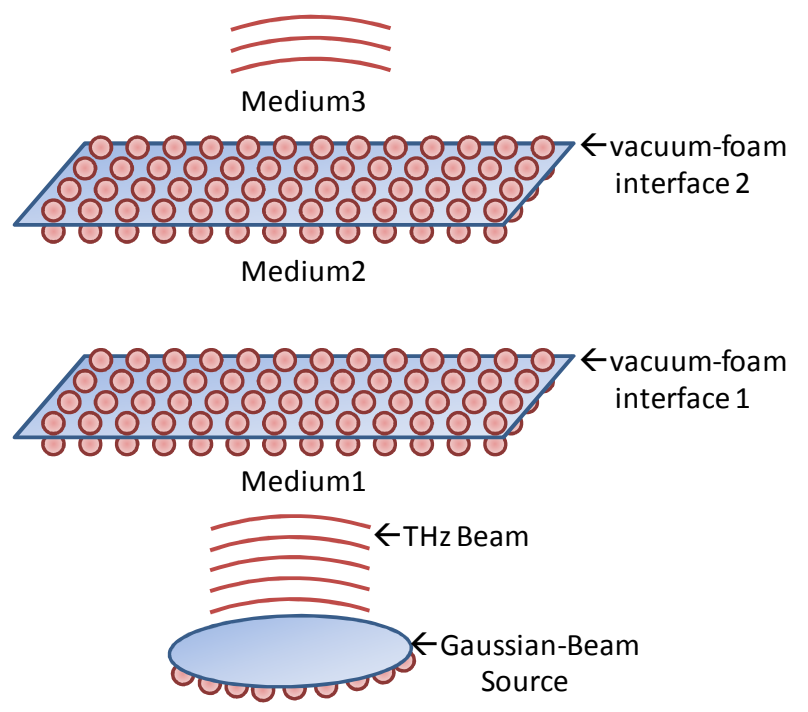

(a)

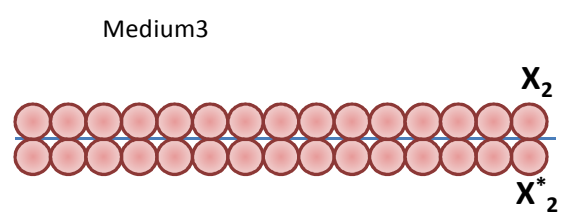

Medium2

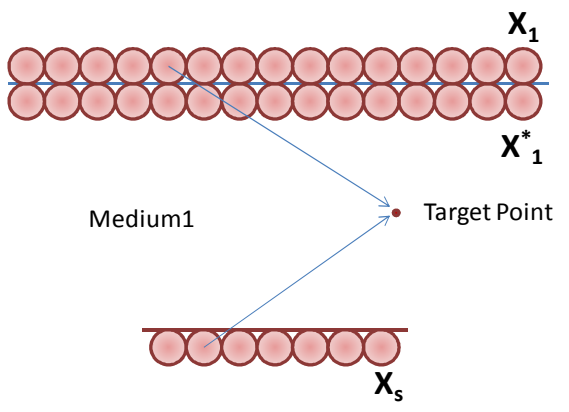

(b)

Figure 1.22 Schematic for modeling a three-medium problem by DPSM - (a) 3D depiction of spherical point sources on both sides of every interface and the lower side of the source, (b) 2D depiction of the DPSM model and the variable names assigned to the interface layers

Using electric field condition of equation (1.88), for a vertically polarized Gaussian beam the boundary condition vector $\mathbf{E}_{\mathrm{SG}(3 \mathrm{~N} \times 1)}$ for $N$ point sources is defined as:

$$
\left.\mathbf{E}_{\mathrm{sG}(3 \mathrm{~N} \times 1)}=\left\{\begin{array}{lllllll}
\left\{E\left(r_{1}, 0\right)\right. & 0 & 0
\end{array}\right\} \quad \ldots \quad\left\{E\left(r_{N}, 0\right) \quad 0 \quad 0\right\}\right\}^{\mathbf{T}}
$$

The boundary condition at the target points on the front face of a circular source gives: 


$$
\mathbf{E F}_{\mathrm{SS}} \mathbf{X}_{\mathrm{S}}=\mathbf{E}_{\mathrm{SG}}
$$

In which $\mathbf{E F}_{\mathrm{ss}(3 \mathrm{~N} \times 3 \mathrm{~N})}$ is the matrix for computing the electric field at the target points placed at the source surface due to the source point sources and is defined as:

$$
\begin{aligned}
& \mathbf{E F}_{\mathrm{ss}(3 \mathrm{~N} \times 3 \mathrm{~N})}= \\
& {\left[\begin{array}{ccccc}
\mathbf{E M}_{1,1} & \mathbf{E M}_{1,2} & \ldots & \mathbf{E M}_{1, \mathrm{~N}-1} & \mathbf{E M}_{1, \mathrm{~N}} \\
\mathbf{E M}_{2,1} & \mathbf{E M}_{2,2} & \ldots & \mathbf{E M}_{2, \mathrm{~N}-1} & \mathbf{E M}_{2, \mathrm{~N}} \\
\vdots & \vdots & \ddots & \vdots & \vdots \\
\mathbf{E M}_{\mathrm{N}-1,1} & \mathbf{E M}_{\mathrm{N}-1,2} & \ldots & \mathbf{E M}_{\mathrm{N}-1, \mathrm{~N}-1} & \mathbf{E M}_{\mathrm{N}-1, \mathrm{~N}} \\
\mathbf{E M}_{\mathrm{N}, 1} & \mathbf{E M}_{\mathrm{N}, 2} & \ldots & \mathbf{E M}_{\mathrm{N}, \mathrm{N}-1} & \mathbf{E M}_{\mathrm{N}, \mathrm{N}}
\end{array}\right]}
\end{aligned}
$$

In equation (1.122) the component $\mathbf{E M}_{\mathrm{i}, \mathrm{j}}$ is the matrix from equation (1.119) used to compute the electric field at target point $i$ due to point source $j$.

Solving equation (1.121) for point source strength vectors the created potential field due to $\mathrm{n}_{\mathrm{p}}$ number of point sources at any desired target point in the domain is given by:

$$
A_{j}\left(\boldsymbol{x}_{t}\right)=\sum_{m=1}^{n_{p}} J_{j m} A_{j m}=\sum_{m=1}^{n_{p}} J_{j m} \frac{\exp \left(-i k r_{m}\right)}{r_{m}}
$$

In which $J_{j m}$ is the $m$-th point source strength in direction $j$ and $r_{m}$ is the distance of the $m$-th point source from the target point $\boldsymbol{x}_{t}$. The electric field is also computed by superposing the electric field vectors generated by $n_{p}$ number of point sources and using equation (1.119) for each point source.

The Magnetic field vector at target point $\boldsymbol{x}_{t}$ using equation (1.93) is given by: 


$$
\begin{aligned}
& B_{1}\left(\boldsymbol{x}_{t}\right)=\sum_{m=1}^{n_{p}} B_{1 m}=\sum_{m=1}^{n_{p}} \frac{\exp \left(-i k r_{m}^{n}\right)}{\left(r_{m}^{n}\right)^{2}}\left[i k+\frac{1}{r_{m}^{n}}\right]\left(J_{2 m} x_{3 m}^{t}-J_{3 m} x_{2 m}^{t}\right) \\
& B_{2}\left(\boldsymbol{x}_{t}\right)=\sum_{m=1}^{n_{p}} B_{2 m}=\sum_{m=1}^{n_{p}} \frac{\exp \left(-i k r_{m}^{n}\right)}{\left(r_{m}^{n}\right)^{2}}\left[i k+\frac{1}{r_{m}^{n}}\right]\left(J_{3 m} x_{1 m}^{t}-J_{1 m} x_{3 m}^{t}\right) \\
& B_{3}\left(\boldsymbol{x}_{t}\right)=\sum_{m=1}^{n_{p}} B_{3 m}=\sum_{m=1}^{n_{p}} \frac{\exp \left(-i k r_{m}^{n}\right)}{\left(r_{m}^{n}\right)^{2}}\left[i k+\frac{1}{r_{m}^{n}}\right]\left(J_{1 m} x_{2 m}^{t}-J_{2 m} x_{1 m}^{t}\right)
\end{aligned}
$$

In which $\left(x_{1 m}^{t}, x_{2 m}^{t}, x_{3 m}^{t}\right)$ is the position vector from the $m$-th point source to the target point $\boldsymbol{x}_{t}$.

The DPSM model solution for the Gaussian beam propagation in vacuum is obtained by solving equation (1.121) and the results are compared with the theoretical predictions (see equation (1.88) ). In order to model a beam with $w_{0}=3 \mathrm{~mm}\left(w_{0}\right.$ is the half beam waist) a circular source with radius $3.6 \mathrm{~mm}$ is considered, number of point sources is chosen in such a way that the average distance between the neighboring points are less than $\lambda / \pi$.

Figure 1.23 shows the theoretical wave form for the electric field (obtained from equation (1.88)) and the variation of its magnitude on the central axis of the beam as a function of the perpendicular distance $(\mathrm{z})$ of the point from the source.

Figure 1.24 shows the same plot computed by DPSM when the Frequency is 0.5 THz. Note that the Electric field magnitude shows some oscillations in Figure 1.24 while the theoretical curve of Figure 1.23 does not show it. This is because the theoretical electric field is generated by a source of infinite radius that produces a Gaussian beam whose strength decays exponentially to zero from the source center while the curve of Figure 1.24 is generated by a finite sized source which produces a Gaussian beam that is 
truncated at its circular periphery. The source radius is then increased from $3.6 \mathrm{~mm}$ to 4.8 $\mathrm{mm}$ to investigate if for the bigger source the computed field is closer to the theoretical curve. It should be noted that for the bigger source the truncated energy of the Gaussian beam is less in comparison to that for the smaller source. Figure 1.25 shows the computed electric field that conforms better with the theoretical curve. It is interesting to note that the truncation of the Gaussian profile at the source periphery produces oscillations in the electric field amplitude along the central axis of the beam as shown in Figure 1.24 and Figure 1.25.

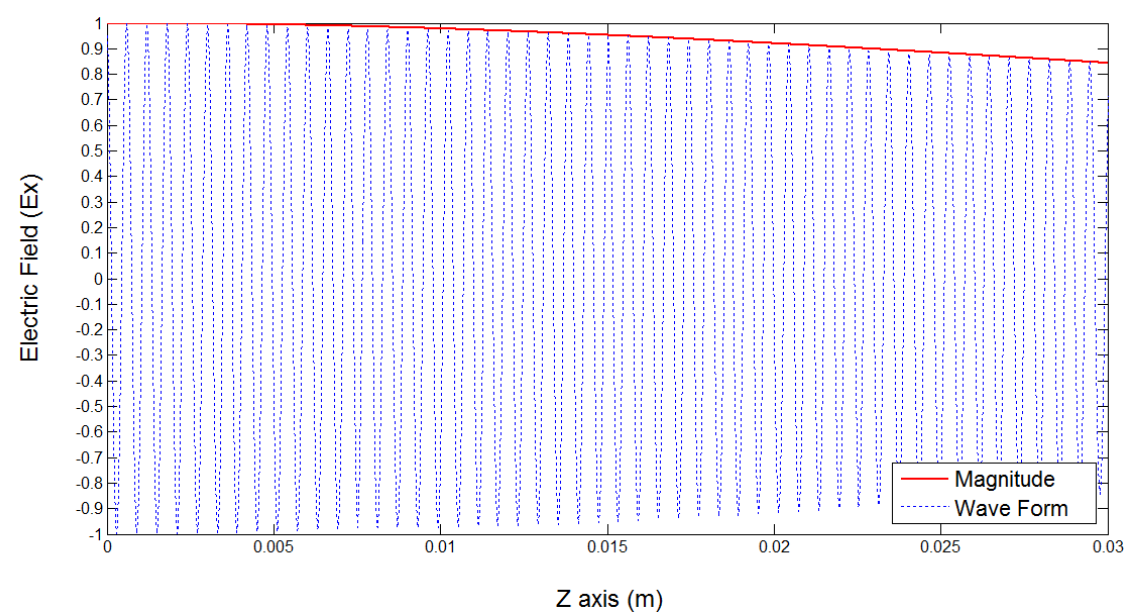

Figure 1.23 Axial electric field in front of a Gaussian beam emitter at 0.5 THz frequency (Analytical solution). 


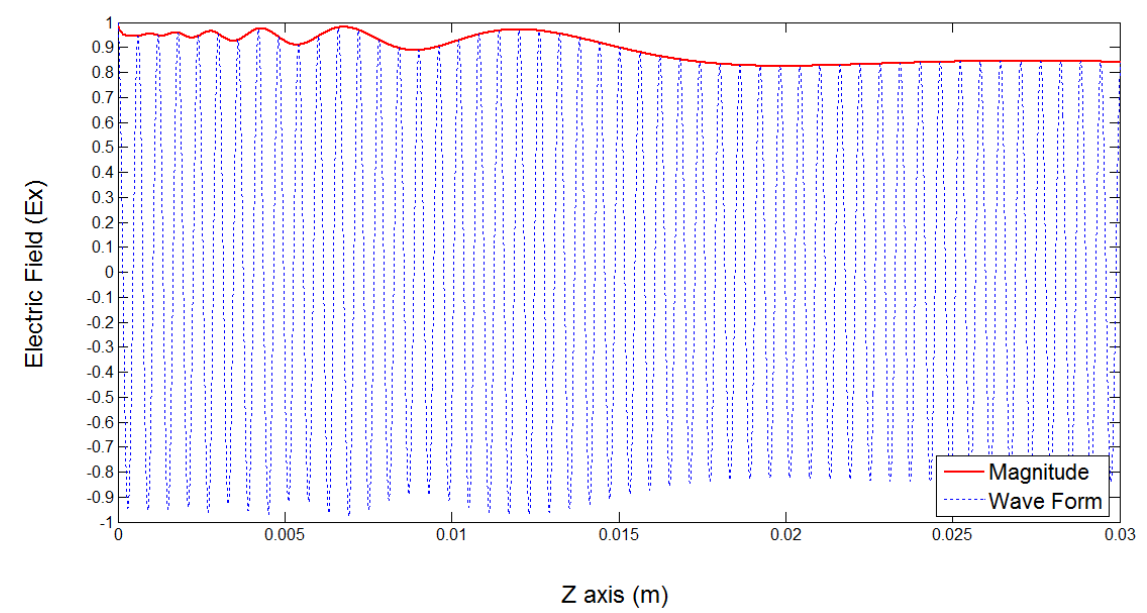

Figure 1.24 Axial electric field in front of a truncated Gaussian beam emitter at 0.5 THz frequency (DPSM solution for circular source of radius $3.6 \mathrm{~mm}$ ).

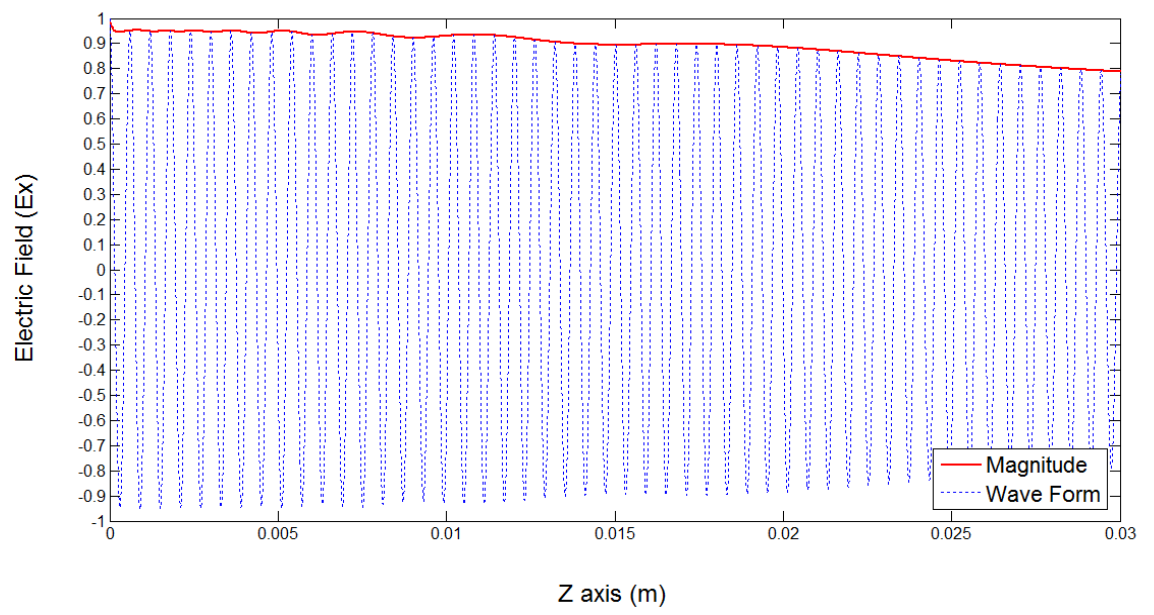

Figure 1.25 Axial electric field in front of a truncated Gaussian beam emitter at $0.5 \mathrm{THz}$ frequency (DPSM solution for circular source of radius $4.8 \mathrm{~mm}$ ).

Figure 1.26 shows the comparison between the theoretical curve for the Gaussian beam and the DPSM generated curve for the truncated Gaussian beam. They show almost perfect matching of both amplitude and phase between the theoretical and DPSM results in spite of the finite dimension of the source for the DPSM results. 
Figure 1.27 compares the complete electric field generated theoretically and by the DPSM analysis in front of the source. Two fields look almost identical.

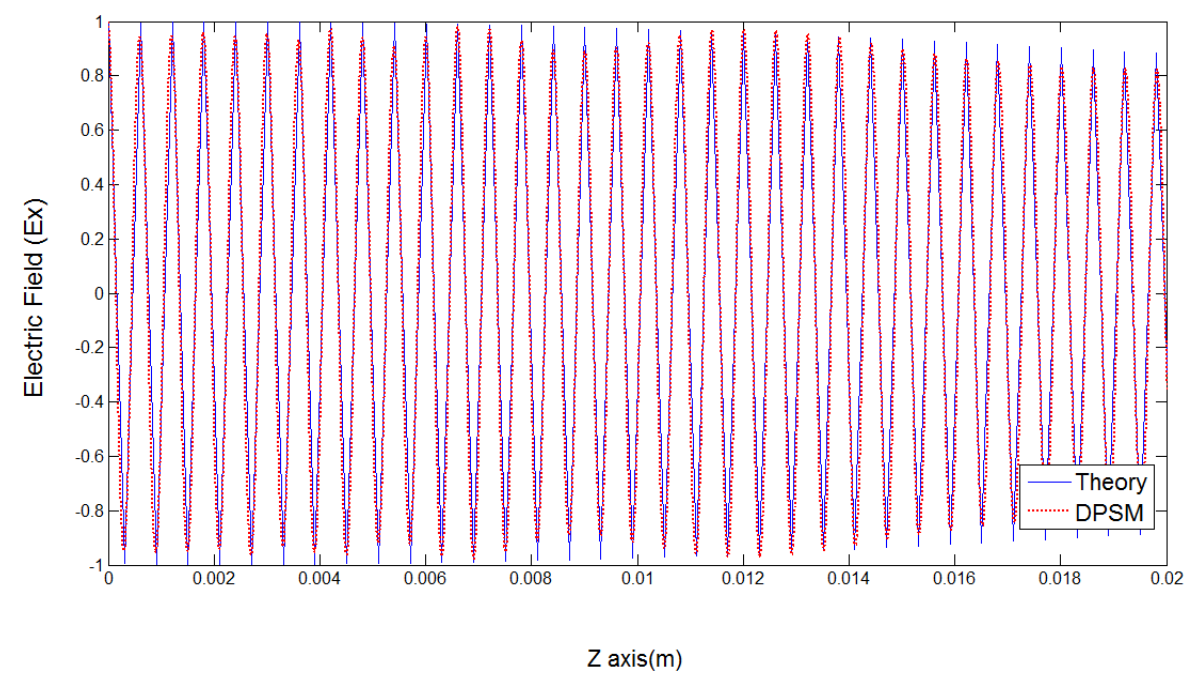

Figure 1.26 Comparison between theoretical and DPSM generated electric fields for Gaussian and truncated Gaussian Beams (circular source of radius $=4.8 \mathrm{~mm}$ for DPSM analysis), frequency $=0.5 \mathrm{THz}$.

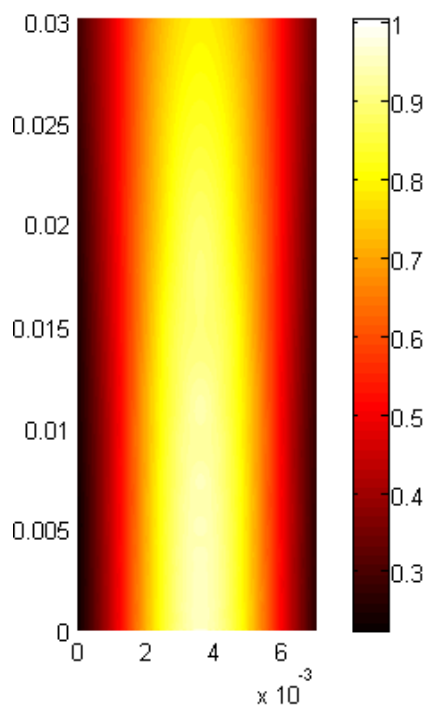

(a)

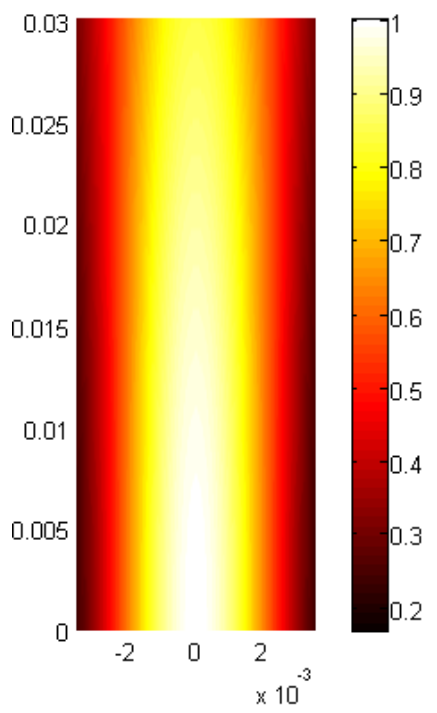

(b)

Figure 1.27 Generated Electric Fields for Gaussian distribution of the source strength (frequency $=0.5 \mathrm{THz})$ : $(\mathrm{a})$ DPSM Solution (circular source of radius $=4.8 \mathrm{~mm})$, (b) Theoretical Solution. 
These comparisons between the DPSM results and the theoretical solution (Figure 1.26 and Figure 1.27) prove that the DPSM is a reliable technique for modeling THz beams.

\subsubsection{DPSM in layered structures}

To model a layered Vacuum-Dielectric-Vacuum structure with a Gaussian beam passing through it, three parallel planar surfaces are considered (see Figure 1.22). One surface represents the Gaussian beam source, and the other two surfaces correspond to the Vacuum-Dielectric interfaces. Small spheres representing point sources are distributed near the source and on both sides of every interface. $N$ point sources are placed behind the source surface and $M$ sources are assigned on each side of the interface planes. The emitter or the source is modeled by one set of point sources; its strength vector is denoted as $\mathbf{X}_{\mathrm{s}(3 \mathrm{~N} \times 1)}$. For every interface, two sets of point sources are introduced. Strengths of the point sources placed above the interfaces are denoted by vectors $\mathbf{X}_{1(3 \mathrm{M} \times 1)}$ and $\mathbf{X}_{\mathbf{2}(3 \mathrm{M} \times 1)}$ while those for the point sources placed below the interfaces are denoted by $\mathbf{X}_{1^{*}(3 \mathrm{M} \times 1)}$ and $\mathbf{X}_{2^{*}(3 \mathrm{M} \times 1)}$. If we denote $\mathbf{A}_{1}$ as the vector potential in medium 1 and $\mathbf{A}_{2}$ as the vector potential in medium 2 then the compatibility conditions require (see [26])

$$
\begin{gathered}
\mathbf{A}_{1}=\mathbf{A}_{2} \\
\frac{1}{\mu_{1}} \frac{\partial \mathbf{A}_{1}}{\partial \mathbf{n}}=\frac{1}{\mu_{2}} \frac{\partial \mathbf{A}_{2}}{\partial \mathbf{n}}
\end{gathered}
$$


In equation (1.126) $\mu_{1}$ and $\mu_{2}$ are permeability coefficients of media 1 and 2 , respectively. As shown in Figure 1.22 the target points can be taken anywhere in the domain. The target points are placed at the apex point of every source sphere touching the interface or the boundary plane. The compatibility and boundary conditions at target points in matrix form can be written as:

$$
\begin{gathered}
\mathbf{E F}_{\mathrm{ss}} \mathbf{X}_{\mathrm{s}}+\mathbf{E F}_{\mathrm{s1}} \mathbf{X}_{1}=\mathbf{E}_{\mathrm{SG}} \\
\mathbf{P F}_{1 \mathrm{~s}} \mathbf{X}_{\mathrm{s}}+\mathbf{P} \mathbf{F}_{11} \mathbf{X}_{1}=\mathbf{P} \mathbf{F}_{11^{*}} \mathbf{X}_{1^{*}}+\mathbf{P} \mathbf{F}_{12} \mathbf{X}_{2} \\
\frac{1}{\mu_{\mathrm{v}}}\left[\mathbf{P G}_{1 \mathrm{~s}} \mathbf{X}_{\mathrm{s}}+\mathbf{P} \mathbf{G}_{11} \mathbf{X}_{1}\right]=\frac{1}{\mu_{\mathrm{f}}}\left[\mathbf{P} \mathbf{G}_{11^{*}} \mathbf{X}_{1^{*}}+\mathbf{P} \mathbf{G}_{12} \mathbf{X}_{2}\right] \\
\mathbf{P F}_{22} \mathbf{X}_{2}+\mathbf{P F}_{21^{*}} \mathbf{X}_{1^{*}}=\mathbf{P F}_{22^{*}} \mathbf{X}_{2^{*}} \\
\frac{1}{\mu_{\mathrm{f}}}\left[\mathbf{P G}_{22} \mathbf{X}_{2}+\mathbf{P G}_{21^{1}} \mathbf{X}_{1^{*}}\right]=\frac{1}{\mu_{\mathrm{v}}}\left[\mathbf{P G}_{22^{*}} \mathbf{X}_{2^{*}}\right]
\end{gathered}
$$

In which:

$$
\mathbf{P G}_{1 \mathrm{~s}(3 \mathrm{M} \times 3 \mathrm{~N})}=\left[\begin{array}{ccccc}
\mathbf{G A}_{1,1} & \mathbf{G A}_{1,2} & \ldots & \mathbf{G A}_{1, \mathrm{~N}-1} & \mathbf{G A}_{1, \mathrm{~N}} \\
\mathbf{G A}_{2,1} & \mathbf{G A}_{2,2} & \ldots & \mathbf{G A}_{2, \mathrm{~N}-1} & \mathbf{G A}_{2, \mathrm{~N}} \\
\vdots & \vdots & \ddots & \vdots & \vdots \\
\mathbf{G A}_{\mathrm{M}-1,1} & \mathbf{G A}_{\mathrm{M}-1,2} & \ldots & \mathbf{G A}_{\mathrm{M}-1, \mathrm{~N}-1} & \mathbf{G A}_{\mathrm{M}-1, \mathrm{~N}} \\
\mathbf{G A}_{\mathrm{M}, 1} & \mathbf{G A}_{\mathrm{M}, 2} & \ldots & \mathbf{G A}_{\mathrm{M}, \mathrm{N}-1} & \mathbf{G A}_{\mathrm{M}, \mathrm{N}}
\end{array}\right]
$$

$$
\mathbf{P F}_{1 \mathrm{~s}(3 \mathrm{M} \times 3 \mathrm{~N})}=\left[\begin{array}{ccccc}
\mathbf{P A}_{1,1} & \mathbf{P A}_{1,2} & \ldots & \mathbf{P A}_{1, \mathrm{~N}-1} & \mathbf{P A}_{1, \mathrm{~N}} \\
\mathbf{P A}_{2,1} & \mathbf{P A}_{2,2} & \ldots & \mathbf{P A}_{2, \mathrm{~N}-1} & \mathbf{P A}_{2, \mathrm{~N}} \\
\vdots & \vdots & \ddots & \vdots & \vdots \\
\mathbf{P A}_{\mathrm{M}-1,1} & \mathbf{P A}_{\mathrm{M}-1,2} & \ldots & \mathbf{P A}_{\mathrm{M}-1, \mathrm{~N}-1} & \mathbf{P A}_{\mathrm{M}-1, \mathrm{~N}} \\
\mathbf{P A}_{\mathrm{M}, 1} & \mathbf{P A}_{\mathrm{M}, 2} & \ldots & \mathbf{P A}_{\mathrm{M}, \mathrm{N}-1} & \mathbf{P A}_{\mathrm{M}, \mathrm{N}}
\end{array}\right]
$$


$\mathbf{E F}_{\mathrm{ss}(3 \mathrm{~N} \times 3 \mathrm{~N})}$ is defined in equation (1.122). In equation (1.128) the component $\mathbf{G} \mathbf{A}_{\mathrm{i}, \mathrm{j}}$ is the matrix from equation (1.116) used to compute the potential gradient at target point $i$ due to point source $j . \quad \mathbf{P G}_{1 \mathrm{~s}(3 \mathrm{M} \times 3 \mathrm{~N})}$ is the matrix for computing the potential gradient at interface- 1 target points due to point sources modeling the emitter. In equation (1.129) the component $\mathbf{P} \mathbf{A}_{\mathrm{i}, \mathrm{j}}$ is the matrix from equation (1.108) used to compute the potential vector at target point $i$ due to point source $j . \mathbf{P F}_{1 \mathrm{~s}(3 \mathrm{M} \times 3 \mathrm{~N})}$ is the matrix for computing potential at interface-1 target points due to point sources modeling the emitter. Other matrices of equation (1.127) follow similar rules. It should be emphasized that in (1.127) the Gaussian beam boundary condition follows equation (1.120).

Equation (1.127) in assembled matrix form gives:

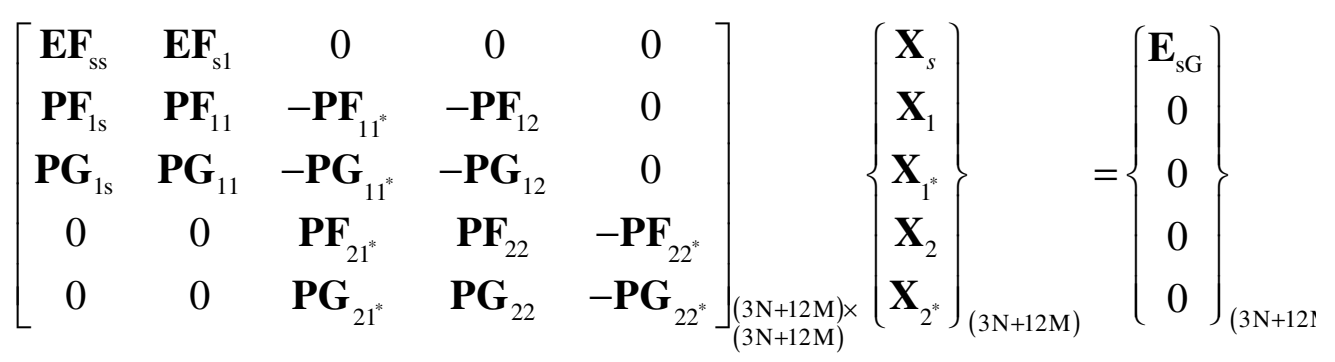

DPSM analysis is carried out for vacuum-foam-vacuum layered structure (V-FV). In [21] the dielectric properties of a packing foam polymer in $\mathrm{THz}$ range were obtained. The same $1.27 \mathrm{~cm}$ thick specimen is modeled here. A homogeneous medium, such as vacuum can also be modeled as a three-layered medium vacuum-vacuum-vacuum $(\mathrm{V}-\mathrm{V}-\mathrm{V})$ as schematically shown in Figure 1.22 (medium $1=$ medium $2=$ medium $3=$ vacuum). For the three-layered medium equation (1.130) is solved using vacuum dielectric properties for all three layers; the frequency is taken as $0.75 \mathrm{THz}$. Figure 1.28 
shows a comparison between the wave-form and its magnitude variation for a vacuumvacuum-vacuum ( $\mathrm{V}-\mathrm{V}-\mathrm{V}$ is the reference medium) and vacuum-foam-vacuum (V-F-V, medium $1=$ medium 3 = vacuum, medium $2=$ foam). Even though there is a small difference between dielectric properties of foam material and vacuum, Figure 1.28 shows that DPSM can detect the effect of small variations in the material properties of the second layer. In order to magnify the distinction between the reference signal (from V-V$\mathrm{V}$ structure) and the sample signal (from V-F-V structure) the power transmittance factor defined in equation (1.84) is used.

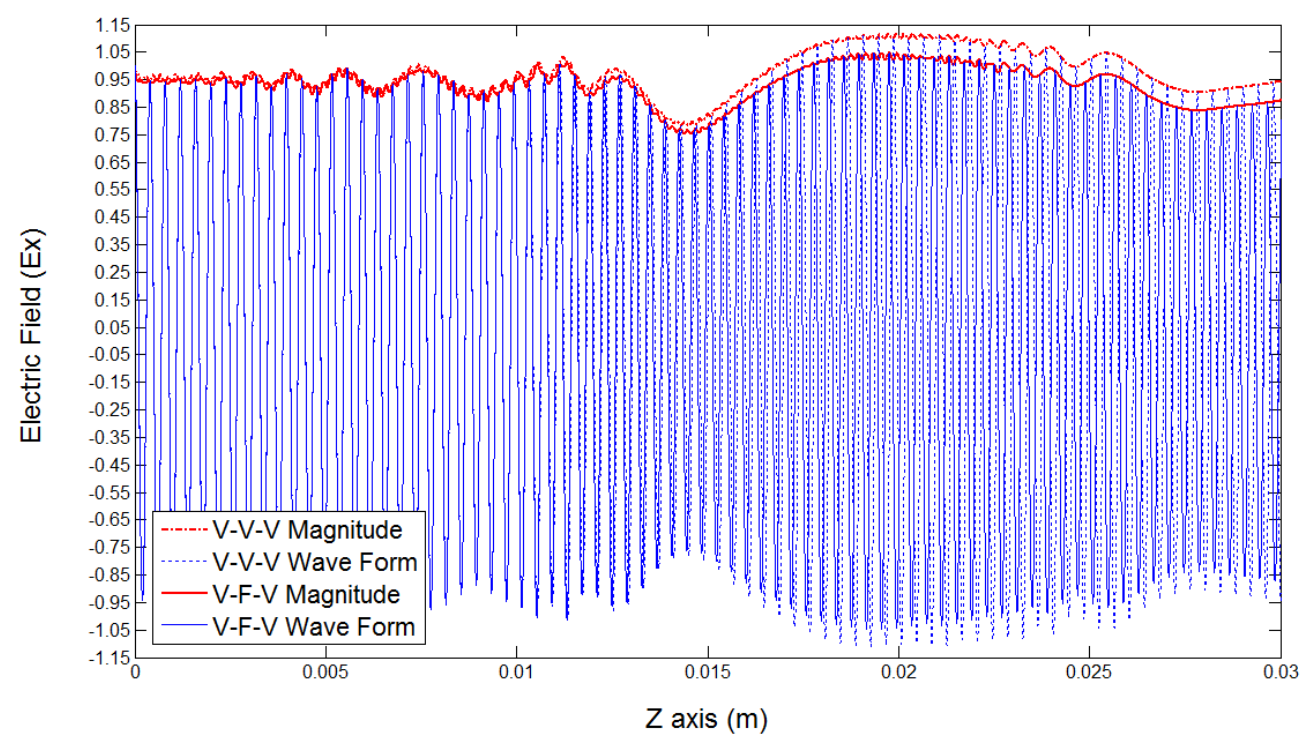

Figure 1.28 Axial electric field in front of a truncated Gaussian beam emitter at 0.75 THz frequency obtained from DPSM modeling (circular source of radius $4.8 \mathrm{~mm}, 29$ Layers of Point Sources are used).

From the sample and reference data in Figure 1.28 at a point located at the receiving signal side of the sample, the PT values for $0.5 \mathrm{THz}, 0.73 \mathrm{THz}$ and $0.75 \mathrm{THz}$ are extracted as follows: 
$20 \log _{10}(96.07 / 96.84)=-0.07$ for $0.5 \mathrm{THz}, 20 \log _{10}(92.092 / 93.90)=-0.1688$ for $0.73 \mathrm{THz}$ and $20 \log _{10}(86.16 / 92.99)=-0.66$ for $0.75 \mathrm{THz}$.

The reason for choosing 0.73 and $0.75 \mathrm{THz}$ frequencies is to see whether DPSM can accurately distinguish between two close frequencies $0.73 \mathrm{THz}$ and $0.75 \mathrm{THz} .0 .5$ $\mathrm{THz}$ is chosen as a point in the low frequency $\mathrm{THz}$ range. As shown in Figure 1.29, results obtained from the DPSM analysis at three frequencies $0.5,0.73$ and $0.75 \mathrm{THz}$ shown by three circular dots fall almost on the experimental curve that can be found in [21]. Clearly DPSM can compute accurately the local peak at $0.73 \mathrm{THz}$ and local dip at $0.75 \mathrm{THz}$.

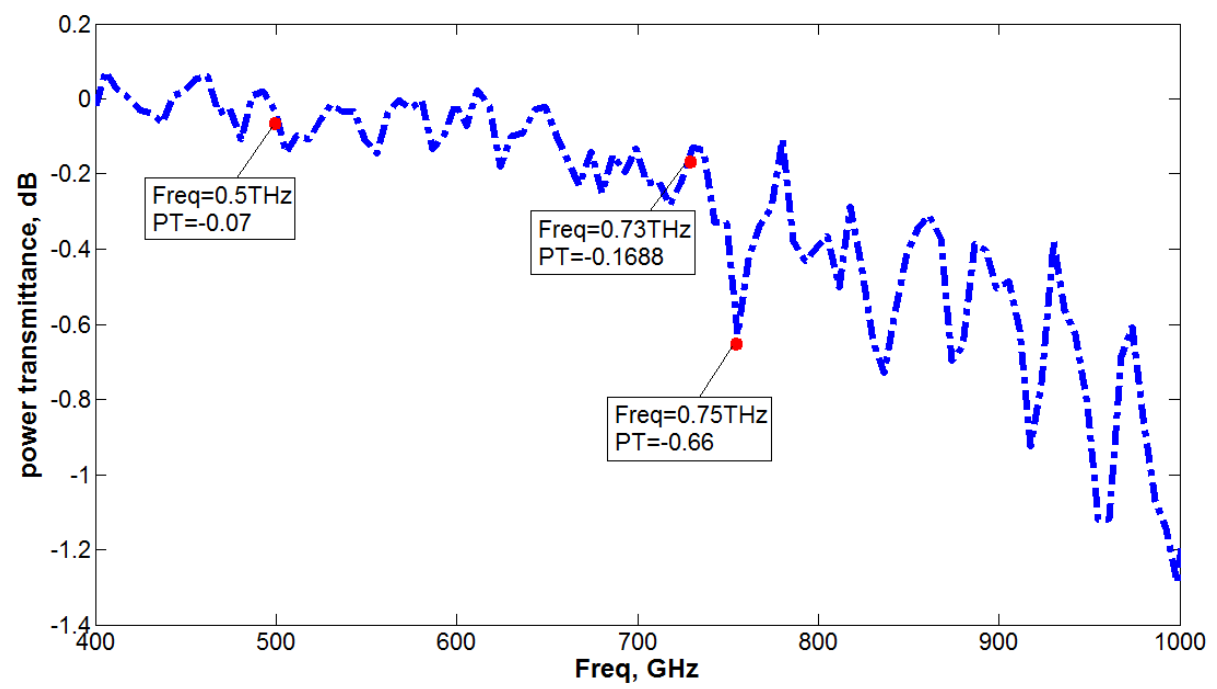

Figure 1.29 Comparison between experimental (see ref [21]) and DPSM predicted PT values, Reference medium is air, sample is solid foam. The dashed-dotted curve is obtained experimentally while the three dots are generated from the DPSM analyses at three frequencies.

\subsubsection{Dielectric scatter modeling in DPSM}

So far for T-rays DPSM has been used to model Gaussian Beam field in homogeneous and multi-layered structures. For Gaussian Beams theoretical solutions are 
available as mentioned above and implemented in DPSM to validate this technique. For multi-layered structure and T-ray interaction, using some standard theoretical methods like Fourier method and ignoring the higher order Fabry-Perot multiple reflections at interfaces, approximate solutions can be obtained.

To emphasize the generality of DPSM another case study is introduced. In Ref. [36] the optical forces on a micro bubble by non-paraxial Gaussian beam were computed using the Finite Difference method. In Ref. [40] using the same method, the scattering field due to high refractive index particles illuminated by focused light beams and the trapping force as a result was computed. In order to calculate the tweezering force, the electric field and magnetic field due to scatterers should be obtained first. By scaling the micro-bubble size modeled in Ref. [36] by a factor of laser wavelength to $\mathrm{THz}$ range wavelength, a $1.2 \mathrm{~mm}$ diameter dielectric sphere was chosen. Three cases were considered, in the first case a dielectric sphere is located $1.5 \mathrm{~mm}$ off-axis position and at $10 \mathrm{~mm}$ vertical distance from the Gaussian source. Case 2 is an axially located sphere with $8 \mathrm{~mm}$ vertical distance from the source and case 3 considers two scatterers, a combination of cases 1 and 2. Dielectric properties of the spheres are assumed $\varepsilon^{\prime}=1.2$ and $\varepsilon^{\prime \prime}=0.036\left(\varepsilon=\varepsilon^{\prime}-i \varepsilon^{\prime \prime}\right.$ is the complex relative permittivity). The relative permeability is assumed to be 1 . The half waist of the Gaussian beam is $w_{0}=3 \mathrm{~mm}$ propagating with frequency $0.5 \mathrm{THz}$. 600 point sources are distributed on each side of the spherical interface.

Referring to Figure 1.30a, the compatibility and boundary conditions at target points in matrix form can be written as: 


$$
\begin{gathered}
\mathbf{E F}_{\mathrm{ss}} \mathbf{X}_{\mathrm{s}}+\mathbf{E F}_{\mathrm{s} 1} \mathbf{X}_{1}+\mathbf{E F}_{\mathrm{s} 2} \mathbf{X}_{2}=\mathbf{E}_{\mathrm{SG}} \\
\mathbf{P F}_{1 \mathrm{~s}} \mathbf{X}_{\mathrm{s}}+\mathbf{P F}_{11} \mathbf{X}_{1}+\mathbf{P F}_{12} \mathbf{X}_{2}=\mathbf{P F}_{11^{*}} \mathbf{X}_{1^{*}} \\
\frac{1}{\mu_{\mathrm{v}}}\left[\mathbf{P G}_{1 \mathrm{~s}} \mathbf{X}_{\mathrm{s}}+\mathbf{P} \mathbf{G}_{11} \mathbf{X}_{1}+\mathbf{P G}_{12} \mathbf{X}_{2}\right]=\frac{1}{\mu_{\mathrm{s} 1}}\left[\mathbf{P G}_{11^{*}} \mathbf{X}_{1^{*}}\right] \\
\mathbf{P F}_{2 \mathrm{~s}} \mathbf{X}_{\mathrm{s}}+\mathbf{P F}_{21} \mathbf{X}_{1}+\mathbf{P F}_{22} \mathbf{X}_{2}=\mathbf{P F}_{22^{*}} \mathbf{X}_{2^{*}} \\
\frac{1}{\mu_{\mathrm{v}}}\left[\mathbf{P G}_{2 \mathrm{~s}} \mathbf{X}_{\mathrm{s}}+\mathbf{P G}_{21} \mathbf{X}_{1}+\mathbf{P G}_{22} \mathbf{X}_{2}\right]=\frac{1}{\mu_{\mathrm{s} 2}}\left[\mathbf{P G}_{22^{*}} \mathbf{X}_{2^{*}}\right]
\end{gathered}
$$

In which $\mu_{\mathrm{v}}, \mu_{\mathrm{s} 1}$ and $\mu_{\mathrm{s} 2}$ are permeability of vacuum, sphere 1 and sphere 2 respectively (it is equal to 1 for this problem). It should be noted that equation (1.131) is for case 3 problem geometry with two scatterers.

Figure $1.30 \mathrm{~b}$ depicts the Gaussian beam electric field magnitude in absence of any scatterer. Figure 1.30c Figure 1.30d and Figure 1.30e are electric fields (fields outside the spheres are shown) corresponding to cases 1 to 3 , respectively. Comparing Figure 1.30e and Figure 1.30d one can see how existence of an off-axis scatterer affects the field around the on-axis scatterer. 


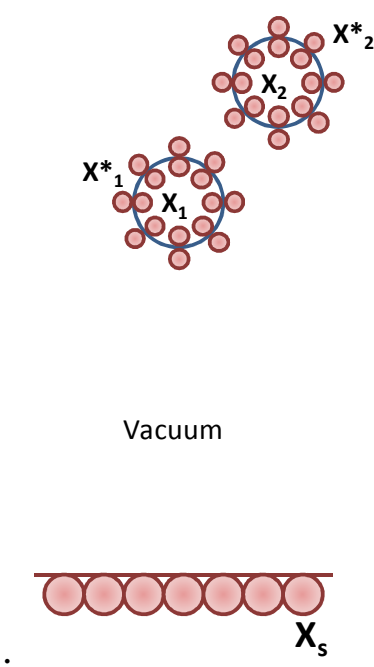

(a)

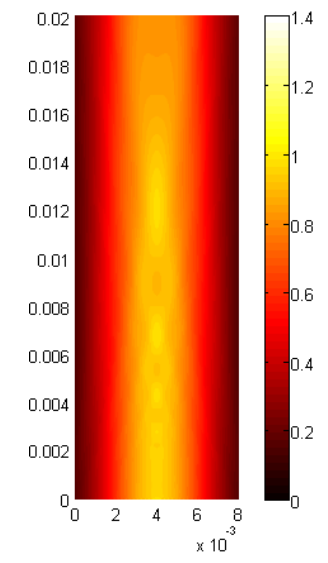

(b)

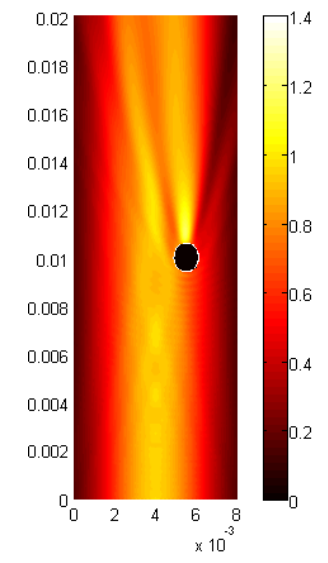

(c)

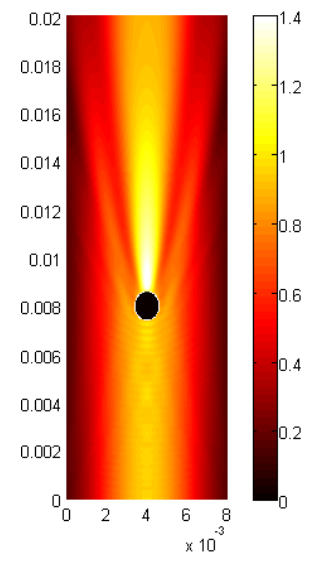

(d)

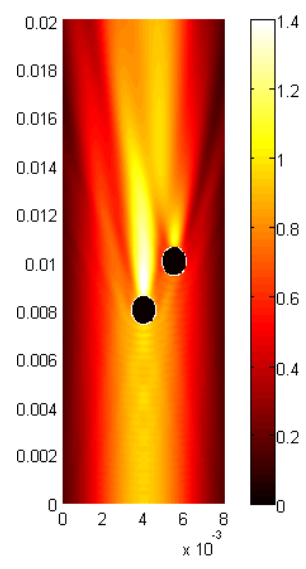

(e)

Figure 1.30 DPSM modeling and interaction between Gaussian beam and dielectric scatterers; (a) 2D schematic depiction of DPSM model, (b) Gaussian Beam in absence of any scatterer, (c) Gaussian Beam in presence of an off-axis single Scatterer, (d) Gaussian Beam in presence of an on-axis single Scatterer, (e) Gaussian Beam in presence of two scatterers 


\subsection{Effect of uniform and non-uniform heat damage}

In section 1.6 the temperature dependency of dielectric properties for blocks heated uniformly for long duration has been investigated experimentally. Is $\mathrm{THz}$ beam capable of distinguishing between the blocks exposed to high temperatures for long and short durations? Very short exposures are not expected to cause any damage and therefore the experimental signals are found to be similar to those of block B1. However, if the block is kept in the oven for a fraction of an hour or a few hours instead of 24 hours then some heat induced damage near the block surfaces are expected while the core of the block might remain undamaged because the oven does not have enough time to heat the inner core of the block. It is analytically investigated here if the $\mathrm{THz}$ beam has enough sensitivity to distinguish between uniformly and non-uniformly heated samples. In this analysis it is assumed that quick heating does not let the material core temperature to increase uniformly, as a result the core is heated less than the outer region.

In the following DPSM is used to model the $\mathrm{THz}$ beam propagation through a block of material which is not heated uniformly and the core is separated by an interface from the outer region. As shown in Figure 1.31 four cases have been chosen. in Case A the entire block has temperature $\mathrm{T} 1$ which is the room temperature. In Case $\mathrm{C}$ the sample is heated uniformly to a maximum temperature and in Cases B and D the core and outer regions are heated at different temperatures. As mentioned above the final objective here 

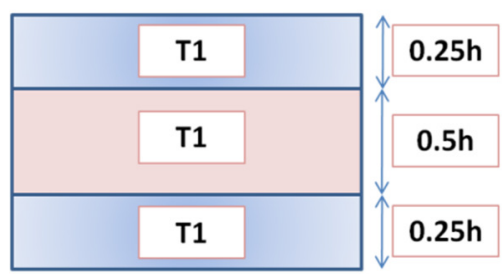

Case A

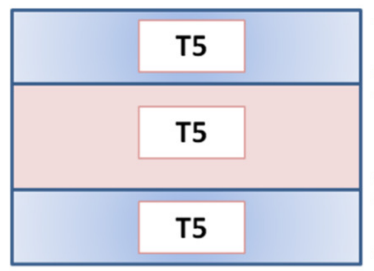

Case C

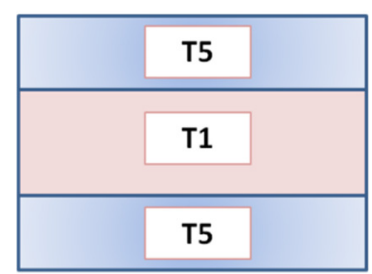

$0.25 h$

$0.5 \mathrm{~h}$

$0.25 \mathrm{~h}$

Case B

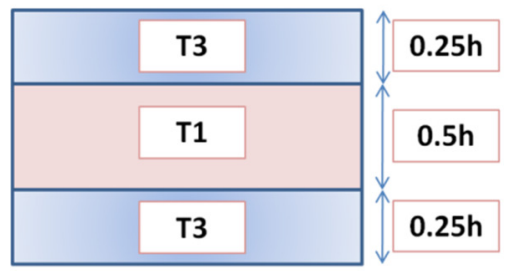

Case D

Figure 1.31 Schematic of samples for different case studies with DPSM ('h' is the sample thickness).

is to investigate whether $\mathrm{THz}$ radiation can differentiate between uniformly and nonuniformly heated specimens.

Figure 1.32 shows a Medium1-Medium2-Medium3-Vacuum domain and a THz beam emitter. A circular emitter of radius $7 \mathrm{~mm}$ is considered. The number of point sources is chosen in such a way that the average distance between the neighboring point sources is $\lambda / \pi$. Circles with radius $7 \mathrm{~mm}$ has been used for modeling the interfaces in a multi-layered structure. Point sources are distributed with average distance of $\lambda / \pi$ between the neighboring sources on both sides of every interface. The sample thickness ' $\mathrm{h}$ ' in Figure 1.31 is assumed to be $25.33 \mathrm{~mm}$ for the DPSM model. The electric field distribution on the $\mathrm{THz}$ emitter surface dictated by equation (1.88) is satisfied along with the compatibility conditions given by equations (1.125) and (1.126) on different interfaces. DPSM takes into account the effect of higher order Fabry-Perot reflections 
inside the material. Thus, DPSM can easily model the interaction between the Gaussian beam and the multilayered problem domain as discussed in detail in section 1.7.

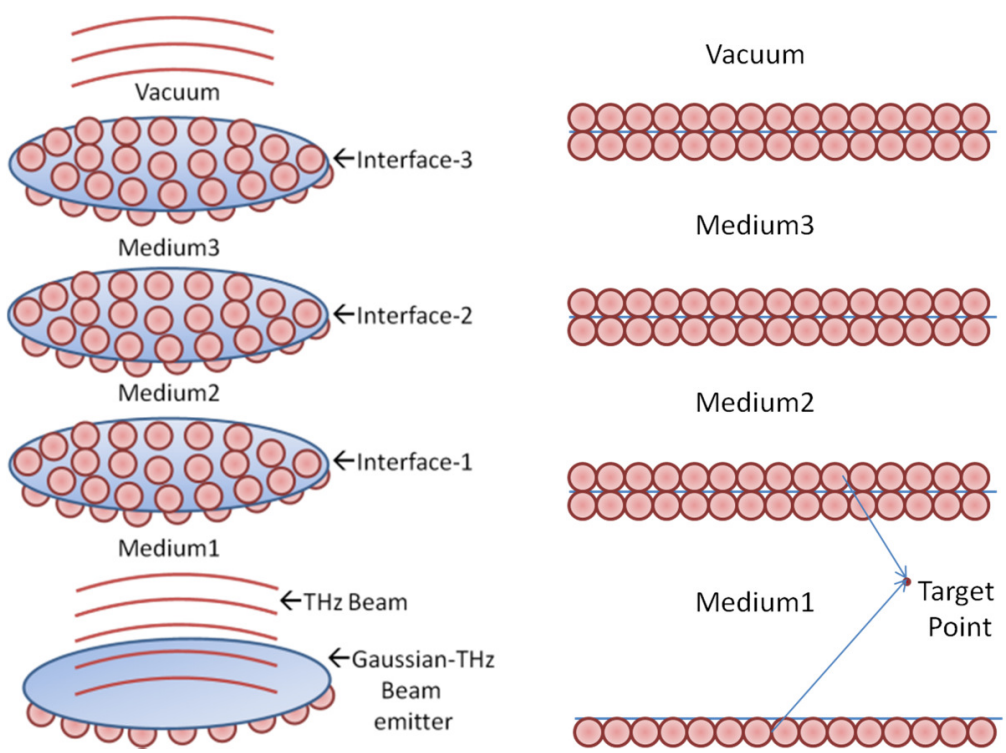

Figure 1.32 Schematic for modeling a four-layered structure with DPSM

The dispersive material properties in Figure 1.20 extracted experimentally for Temperatures T1 to T5 are used for DPSM modeling of different cases (A to D of Figure 1.31). For Case A the DPSM model is built by considering medium $1=$ medium 2 = medium $3=\mathrm{T} 1$, for Case $\mathrm{B}$ medium $1=$ medium $3=\mathrm{T} 5$ and Medium $2=\mathrm{T} 1$. Similar rules apply for other two cases.

Figure 1.33 shows the electric field in front of the $\mathrm{THz}$ emitter generated by the DPSM analysis for the four cases given in Figure 1.31. The computed electric fields look almost identical for these four cases and the attenuation of the signal passing through the material is evident. 


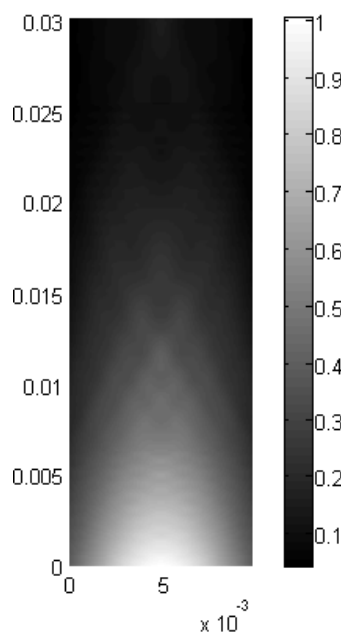

(a)

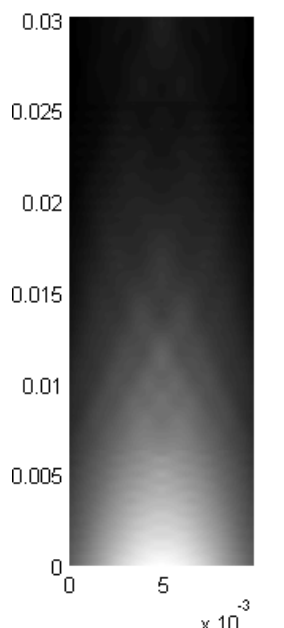

(b)

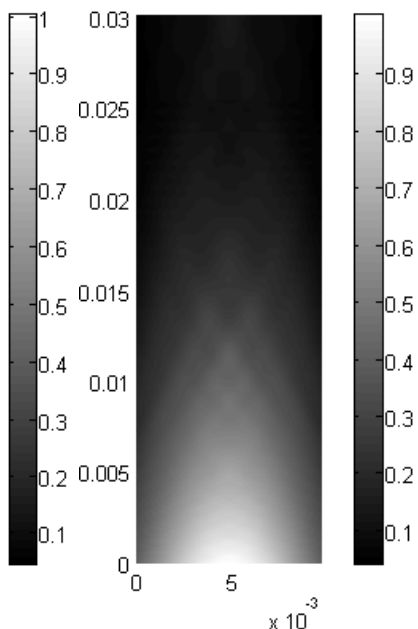

(c)

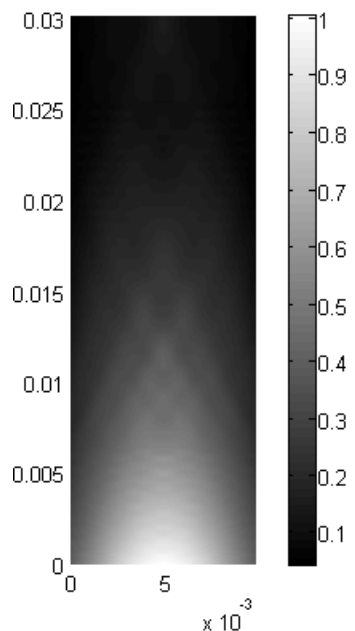

(d)

Figure 1.33 Gaussian beam field generated by DPSM (emitter radius = $7 \mathrm{~mm}$, frequency = $0.22 \mathrm{THz}$ ); (a): Case $A$, (b): Case B, (c): Case C and (d): Case D of Figure 1.31, all dimensions are in meters

Figure 1.34 shows the DPSM generated three wave forms for the electric field on the central axis of the emitter for Cases A, B and C, respectively. Wave forms are plotted as functions of the perpendicular distance $(\mathrm{z})$ of the observation point from the transducer face. Changes in wavelength and amplitude for different cases are evident in this figure although different cases could not be distinguished visually in Figure 1.33.

Figure 1.35 compares the Power transmittance $(\mathrm{PT})$ curves for Cases A to D, PT is chosen in order to magnify the distinction between the reference signal in vacuum and the sample signals for cases A to D. PT in $\mathrm{dB}$ has been defined in equation (1.84).

DPSM modeling results in Figure 1.34 and Figure 1.35 show that the $\mathrm{THz}$ radiation is capable of distinguishing non-uniformly heated samples from uniformly heated ones in addition to detecting the heat induced damage well before its melting point (see Figure 1.20). 


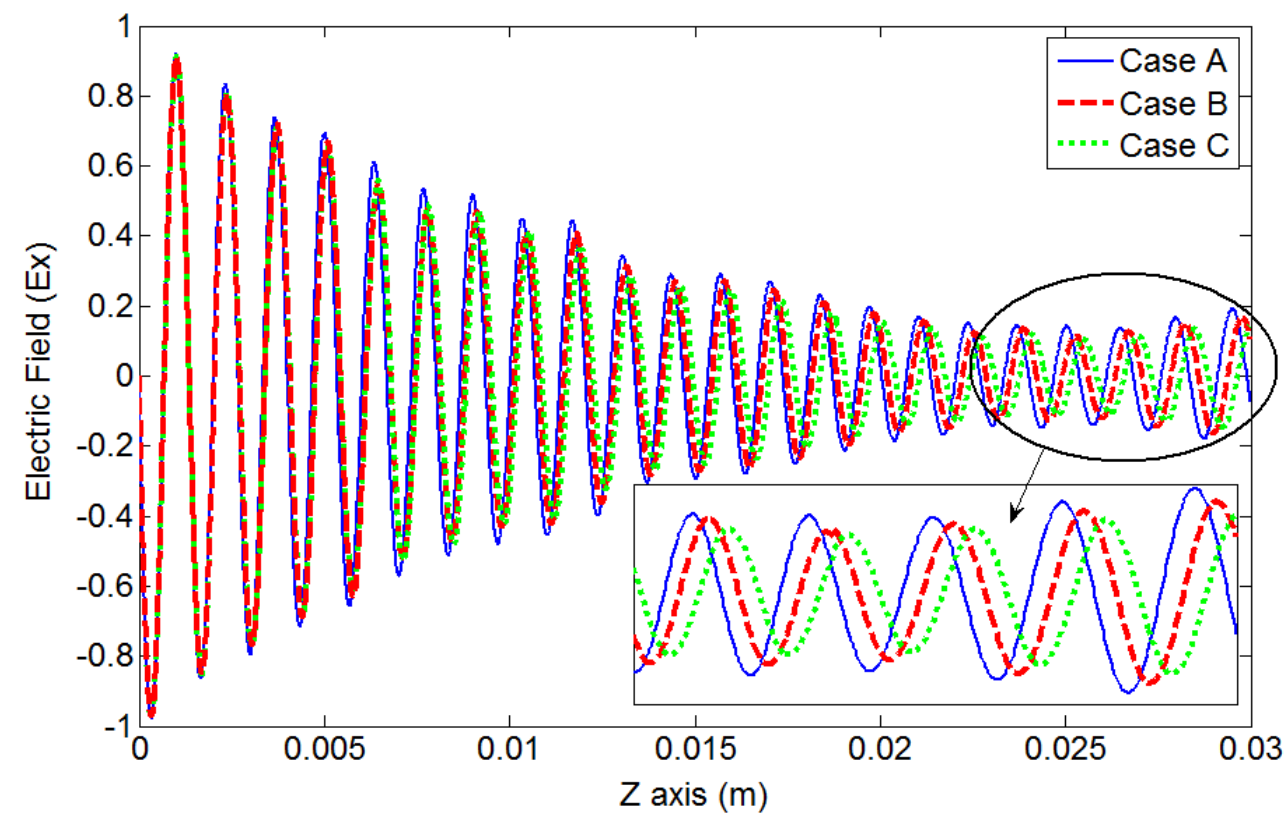

Figure 1.34 Gaussian beam generated axial electric field obtained from DPSM modeling, comparison between Cases A, B and C (emitter and interface radius $=7 \mathrm{~mm}$, Frequency $=0.22 \mathrm{THz}$ ).

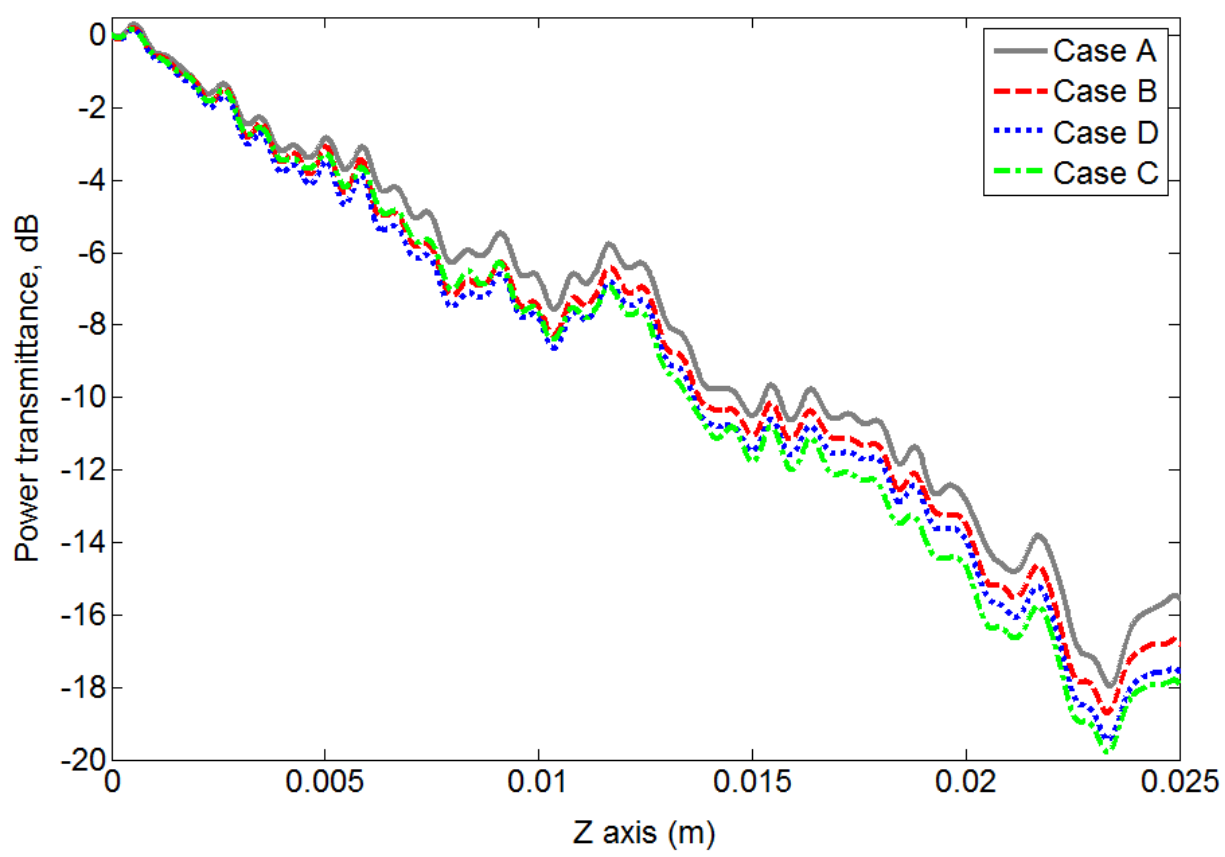

Figure 1.35 Gaussian beam generated axial PT values obtained from DPSM modeling, comparison between Cases A, B, C and D of Figure 1.31 (emitter and interface radius $=7 \mathrm{~mm}$, Frequency $=0.22 \mathrm{THz}$ ). 


\subsection{Summary}

Some NDE applications of $\mathrm{THz}$ radiation are presented in this chapter. It is shown that it can detect both mechanical and heat induced damages in porous tiles through which ultrasonic signals cannot propagate. DPSM Modeling of THz radiation in homogeneous and layered structures is also presented.

In section 1.5 mechanical damages in polymer foam tiles have been detected by THz beams. It is found that one-eighth inch $(\sim 3 \mathrm{~mm})$ diameter through-hole in a $1 \times 1 \mathrm{x}$ 0.5 inch $(25.4 \times 25.4 \times 12.7 \mathrm{~mm})$ polymer foam tile can be detected by a $10 \mathrm{~mm}$ diameter $\mathrm{THz}$ beam if the signal frequency greater than 0.2 or $0.4 \mathrm{THz}$ is used. Choice of frequency depends on the orientation of the hole and more importantly is relevant to the size of the hole, owing to the diffraction limit. For the beam propagating parallel to the hole a dip is observed near $550 \mathrm{GHz}$ frequency in the plot of the ratio of the transmitted energy through the defective tile and the defect-free tile. Numerical FEM simulation is carried out to justify the experimental results. Although air and the polymer have very small difference in their dielectric properties that small difference is enough to detect the cylindrical air inclusion in the polymer tile because of relatively strong scattering of the $\mathrm{THz}$ beam at the interface of these two very similar dielectric materials.

In section 1.6 heat induced damages in polymer foam tiles have been detected by $\mathrm{THz}$ beams. It is found that effective dielectric properties of porous polymer pumice stones are changed as the heat exposure temperatures are raised. A consistent trend with the heat exposure temperature variation is observed in sub-THz frequency. It is shown 
that the material undergoes a significant change in its dielectric properties between $200^{\circ} \mathrm{C}$ and $400^{\circ} \mathrm{C}$, well below its melting point which is near $900^{\circ} \mathrm{C}$.

\subsection{Future Works}

In section 1.8 using DPSM analysis it was numerically shown that $\mathrm{THz}$ radiation is capable of distinguishing non-uniformly heated samples from uniformly heated ones but it has not been proven experimentally yet. In future new experiments should be setup to heat pumice stones for different durations at constant temperatures and investigate whether $\mathrm{THz}$ can distinguish between samples heated for short and long durations. Interactions of $\mathrm{THz}$ beams with cracks inside material should also be investigated in the future. 


\section{CHAPTER 2}

\section{T-DPSM: EXTENSION OF CONVENTIONAL DPSM FOR TRANSIENT ANALYSIS}

\subsection{INTRODUCTION}

Distributed Point Source Method (DPSM) is based on the time harmonic steadystate Green's functions (see [26, 27]). For this reason mostly steady state ultrasonic and electromagnetic problems have been solved by DPSM. So far this method has been used to model phased array transducers [41], relatively complex geometries [42, 43], and homogeneous medium containing finite size scatterers [31, 44] by placing point sources along the boundaries and interfaces. For ultrasonic field modeling DPSM has several advantages over other existing numerical techniques, such as it is a mesh-free technique whereas conventional Finite Element Method requires very small size elements for modeling high frequency ultrasonic problems leading to sometimes computationally impossible to solve problems. Also, in FEM the absorbing boundary conditions require fine tuning of the material properties to successfully avoid spurious reflections from the artificial boundaries while in DPSM there is no need for any such absorbing boundary conditions. DPSM being a mesh-free technique circumvents the problem of requiring small mesh size and can work well at high frequencies (see [28]).

The problem of detection and characterization of floating air bubbles in a liquid medium that is considered here has many applications in materials science and medical fields (see [45-47] ). Although several experimental investigations have been reported in 
the literature, very few publications on the theoretical modeling of the interaction between ultrasonic transducers and cavities inside a fluid medium can be found (see [48]). Interaction between single and double cavities or voids in a liquid and a converging ultrasonic beam generated by a point-focused acoustic transducer with steady state timeharmonic excitation has been modeled by DPSM $[49,50]$. The pressure field generated by a transducer in absence of any bubble gives the baseline field. Any deviation from the baseline field indicates the possibility of the presence of one or more bubbles in the fluid. Comparing the baseline field and the pressure field in presence of bubbles helps us to detect and characterize the air bubbles present in the fluid.

The transient wave propagation in solid and fluid structures is one of many fundamental problems of wave propagation that can be found in text books [51-53]. However, these textbook problems involve only plane, spherical and cylindrical waves interacting with simple structures. It is necessary to follow numerical techniques for modeling wave propagation in complex structures. Finite Element Method can be used to solve transient wave propagation in complex structures [54-56] however it is not well suited to model unbounded media. Spectral Finite Element Method [57-59] and Boundary Element Method $[60,61]$ can be also deployed for transient wave propagation analysis in solid and fluid structures. All these methods require meshing of the problem geometry or its boundaries and interfaces.

As discussed above and in earlier publications [28] it has been established that DPSM is more efficient than FEM for solving three-dimensional steady state ultrasonic problems. Therefore, it will be even a stronger method if it can be extended to solve 
transient problems in an efficient manner. Since DPSM is a Green's function based technique and Green's functions for ultrasonic problems are available in the frequency domain, so far DPSM has been mostly used to solve steady state or harmonic problems. Transient problems can be solved by applying the Fast Fourier Transform (FFT) to the frequency domain response. A shortcoming of this FFT based conventional approach is that it requires solution of several harmonic problems at different frequencies to construct the frequency spectrum which is then inverted to obtain the transient solution as done by Kundu et al. [62].

In this research an alternative way of obtaining the transient solution from the DPSM analysis is presented. It does not require any FFT inversion and is especially useful when the frequency spectrum decays slowly or when only a part of the time history is of interest. This time domain DPSM analysis will be called t-DPSM. The tDPSM predicted results are compared with the Finite element generated results for some simple problems. Then t-DPSM is used to solve a more complex problem - transient solution of the scattering of a focused ultrasonic beam by a spherical void inside the fluid. Changes in the received signal for different void eccentricities are studied. Numerical results are presented in presence of single as well as double cavities. Axisymmetric FEM modeling is also used to solve a circular transducer in absence of any cavity and in presence of a cavity located on the central axis of the transducer. When the cavity is moved from the axis of symmetry of the transducer, the 3D FE model is necessary. 3D FE modeling is too time-consuming and many computers still cannot handle it. The versatility and advantages of t-DPSM method are evident for these problems. 


\subsection{A Brief Review of Distributed Point Source Method (DPSM)}

The DPSM theory has been discussed in detail in earlier publications [26-29, 63] . In DPSM a finite size transducer is modeled by a finite number of point sources [64]. The total ultrasonic field generated by the transducer is the summation of the ultrasonic fields produced by all these point sources. The point sources are placed very close to the boundary and interfaces, but not on the interface or the boundary. This is necessary to avoid the singularity of the computed field at the point source location. The distance between the point source center and the interface is set equal to $r_{S}=\lambda / 2 \pi$ ( $\lambda$ is the wave length) and that between two neighboring point sources is equal to $\lambda / \sqrt{2 \pi}$ or $\lambda / 3$ for proper convergence [65]. Any anomaly present in the structure can be modeled by placing additional layers of point sources at the boundary or the interface of the anomaly.

\subsection{Matrix Representation for Transducer Cavity Interaction in a Fluid Medium}

The schematic diagram of the problem geometry involving a spherical cavity inside a fluid medium and a finite size concave transducer is shown in Figure 2.1. The transducer is modeled by a layer of $n$ point sources. $\mathbf{A}_{\mathrm{S}}$ is the $(n \times 1)$ source strength vector of the point sources modeling the acoustic source or the transducer. These sources contribute to the incident ultrasonic field in the fluid. The cavity is modeled by another layer of $m$ point sources. $\mathbf{A}_{1}$ is the ( $\left.m \times 1\right)$ source strength vector modeling the cavity. The point sources modeling the cavity generate the scattered field. The total ultrasonic field at any point is the summation of the contributions of these two layers of point sources. $\mathbf{A}_{\mathrm{S}}$ and $\mathbf{A}_{1}$ are the unknown vectors of source strength that are obtained by satisfying the boundary conditions The centers of the point sources are located at a distance $r_{S}$ from the 
boundaries. The boundary conditions are satisfied at the apex points where the small spheres touch the boundaries. The number of point sources and the number of discrete boundary points where the boundary conditions are satisfied are the same.

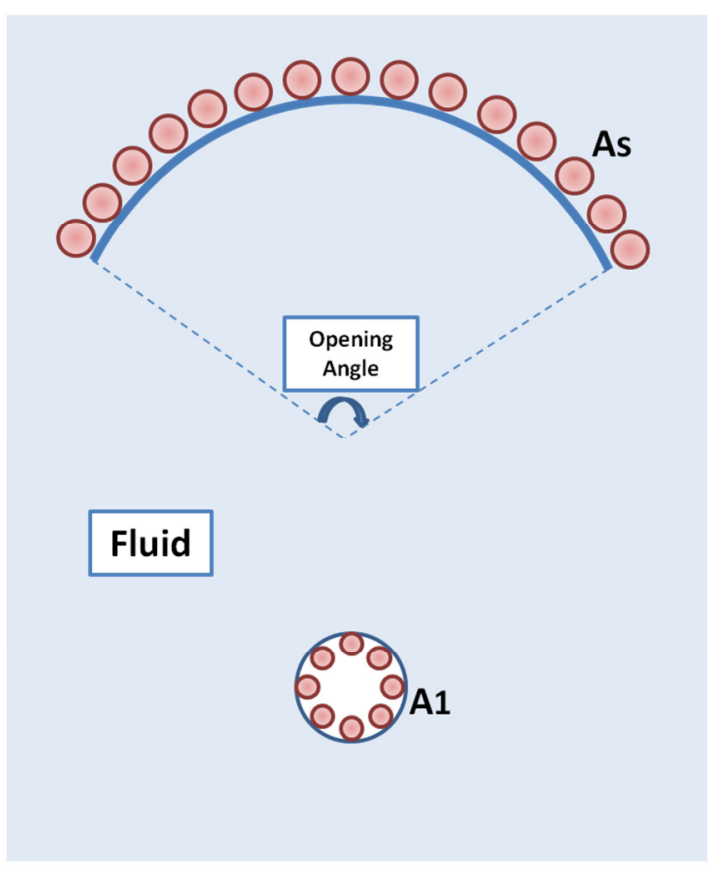

Figure 2.1 DPSM model for a spherical cavity inside a fluid containing a point-focused ultrasonic transducer.

The acoustic pressure at any given set of points inside the fluid can be expressed in matrix form. These points are called the target points. It is assumed that the transducer is force controlled and the pressure on the transducer surface is known. If the pressure on the transducer face is assumed to be $\mathbf{P}_{\mathbf{S O}}$ then the boundary conditions on the transducer surface can be written as

$$
\mathbf{M}_{\mathrm{sS}} \mathbf{A}_{\mathrm{s}}+\mathbf{M}_{\mathrm{s} 1} \mathbf{A}_{1}=\mathbf{P}_{\mathrm{S} 0}
$$


In equation (2.1) $\mathbf{M}_{\mathrm{ss}}$ and $\mathbf{M}_{\mathrm{s} 1}$ are two matrices of dimensions $n \times n$ and $n \times m$, respectively. The elements of these matrices when multiplied by the source strengths produce pressure on the transducer surface. These matrix elements are functions of the Green's functions, given in the literatures [26, 27]. For the above matrices, the subscript 's' denotes the source or the transducer and subscript ' 1 ' denotes cavity. For example, $\mathbf{M}_{\mathrm{s} 1}$ is the matrix required to calculate the pressure on the transducer surface target points due to cavity source points and is defined as:

where,

$$
\mathbf{M}_{\mathrm{s} 1}=\left[\begin{array}{ccccc}
g_{1,1} & g_{1,2} & \cdots & g_{1, m-1} & g_{1, m} \\
g_{2,1} & g_{2,2} & \ldots & g_{2, m-1} & g_{2, m} \\
\vdots & \vdots & \ddots & \vdots & \vdots \\
g_{n-1,1} & g_{n-1,2} & \cdots & g_{n-1, m-1} & g_{n-1, m} \\
g_{n, 1} & g_{n, 2} & \cdots & g_{n, m-1} & g_{n, m}
\end{array}\right]
$$

$$
g_{i, j}=\frac{\exp \left(-i k_{f} r_{i, j}\right)}{r_{i, j}}
$$

In equation (2.3) $k_{f}=\omega / c_{f}$ is the wave number, $c_{f}$ is the acoustic wave speed in the fluid and $\omega$ is the angular frequency. $r_{i, j}$ is the distance between the source point $i$ and the target point $j$. gives the pressure on the transducer surface due to transducer source points. On the cavity boundary, the pressure should be zero. Therefore,

$$
\mathbf{M}_{1 \mathrm{~s}} \mathbf{A}_{\mathrm{s}}+\mathbf{M}_{11} \mathbf{A}_{1}=0
$$


$\mathbf{M}_{1 \mathrm{~s}}$ and $\mathbf{M}_{11}$ are two matrices of dimensions $m \times n$ and $m \times m$ respectively. $\mathbf{M}_{1 \mathrm{~s}}$ when multiplied by $\mathbf{A}_{\mathrm{s}}$ gives the pressure on the cavity surface target points due to point sources on the transducer surface and $\mathbf{M}_{11} \mathbf{A}_{1}$ gives the pressure on the cavity surface due to point sources placed near the cavity surface. Equations (2.1) and (2.4) can be combined to obtain,

$$
\left[\begin{array}{ll}
\mathbf{M}_{\mathrm{ss}} & \mathbf{M}_{\mathrm{s} 1} \\
\mathbf{M}_{1 \mathrm{~s}} & \mathbf{M}_{11}
\end{array}\right]_{(n+m) \times(n+m)}\left\{\begin{array}{l}
\mathbf{A}_{\mathbf{s}} \\
\mathbf{A}_{1}
\end{array}\right\}_{(n+m)}=\left\{\begin{array}{c}
\mathbf{P}_{\mathrm{s} 0} \\
0
\end{array}\right\}_{(n+m)}
$$

Equation (2.5) can be written in a simpler form,

$$
[\mathbf{M T}]\{\mathbf{\Omega H}\}=\{\mathbf{P}\}
$$

Note that Equations (2.5) and (2.6) are identical equations but written in two different ways. These equations satisfy simultaneously the boundary conditions on the cavity surface and the transducer surface. This system of equations (2.6) has a unique solution. The number of point sources needed for the ultrasonic field computation is obtained by satisfying the convergence criterion [26] that the spacing between two neighboring point sources should be less than $\lambda / \sqrt{2 \pi}$ or $\lambda / 4$.

Solving equation (2.6) the source strength vectors for the complete problem geometry are obtained,

$$
\{\mathbf{\Omega H}\}=[\mathbf{M T}]^{-1}\{\mathbf{P}\}
$$


Matrix [MT] is a well-conditioned matrix and its inversion can be carried out without any difficulty.

\subsection{Transient DPSM (t-DPSM) Formulation}

Green's functions used in conventional DPSM are based on the time harmonic Helmholtz equation for fluids. In order to carry out the time domain analysis, Green's function satisfying Helmholtz equation in time domain should be implemented. Consider a point source located at Xs exciting with function $f(t)$, the Helmholtz equation ([26], [52]) gives:

$$
\nabla^{2} G-\frac{1}{c_{f}^{2}} \frac{\partial^{2} G}{\partial t^{2}}=f(t) \delta|\mathbf{X}-\mathbf{X s}|
$$

In which $c_{f}$ is the acoustic wave speed in the fluid and $\delta$ is the Dirac delta function. The solution of equation (2.8) is of the form

$$
G(\mathbf{X}, t ; \mathbf{X s})=-\frac{1}{4 \pi|\mathbf{X}-\mathbf{X s}|} f\left(t-t_{r e t}\right)
$$

In which $t_{r e t}$ is the retarded time defined as:

$$
t_{r e t}=\frac{|\mathbf{X}-\mathbf{X s}|}{c_{f}}
$$

From this solution one can see that it takes $t_{\text {ret }}$ time for an excitation at $\mathbf{X s}$ to reach target point $\mathbf{X s}$, or at time $t$ the target point receives the excitation occurred at time $t-t_{\text {ret }}$. The main step for the time dependent analysis with DPSM is assuming a time dependent point source strength in t-DPSM in contrast to steady state DPSM in which the 


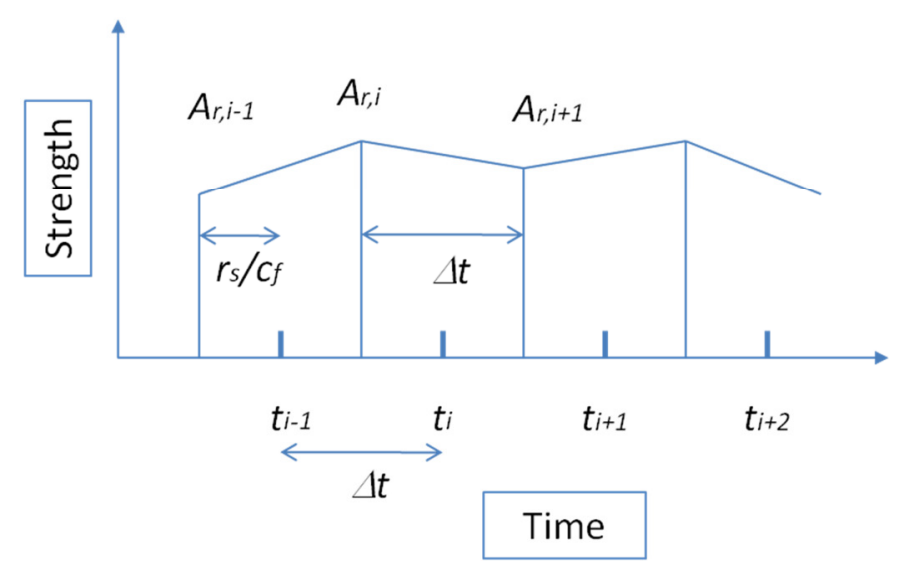

(a)

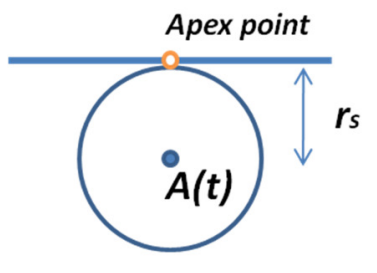

(b)

Figure 2.2 A time dependent point source strength function by linear interpolation; (b): Point source schematic

point source strength is a constant. Consequently the main objective is to solve for point source values at every time step.

Let us consider a single point source as shown figure Figure 2.2b. From equation (2.9) the pressure value at the apex point due to excitation of the point source is obtained as:

$$
P(t)=-\frac{1}{4 \pi r_{s}} A\left(t-\frac{r_{s}}{c_{f}}\right)
$$

In equation (2.11) $A(t)$ is a time dependent function denoting the point source strength. $r_{s}$ is the point source sphere radius (see Figure 2.2b). As shown in Figure 2.2a function $A(t)$ is assumed to vary linearly over a time step $\Delta t$. Now we need to discretize the total time interval with time steps $\Delta t$. Since it takes a retarded time $r_{s} / c_{f}$ for any excitation to travel from the point source to the apex point our objective is to find the point source 
value at $t_{i}-r_{s} / c_{f}$ in which $t_{i}$ is the $i$-th time step at which the boundary condition should be satisfied. From equation (2.11) the apex point pressure at time $t_{i}$ can be written as:

$$
P\left(t_{i}\right)=-\frac{1}{4 \pi r_{s}} A\left(t_{i}-\frac{r_{s}}{c_{f}}\right)=-\frac{1}{4 \pi r_{s}} A_{r, i}
$$

As shown in figure Figure 2.2 at each time step $t_{i}$ the point source strength $A_{r, i}$ is calculated and by using a linear interpolation the time dependent excitation function for each point source is acquired. Now if $r_{t}$ is the distance between the point source and any target point like neighboring apex points, it takes the retarded time $t_{\text {ret }}=r_{t} / c_{f}$ for the excitation to reach the target point. The pressure at the target point becomes:

$$
\begin{aligned}
& P\left(t_{i}\right)=-\frac{1}{4 \pi r_{t}} A\left(t_{i}-\frac{r_{t}}{c_{f}}\right)=-\frac{1}{4 \pi r_{t}} A\left(t_{i}-t_{r e t}\right) \\
& =-\frac{1}{4 \pi r_{t}}\left[C_{i}+K_{i} A_{r, i}\right]
\end{aligned}
$$

In which constants $C_{i}$ and $K_{i}$ are extracted using linear interpolation of $A(t)$.

if $\left(t_{r e t}-r_{s} / c_{f}\right)<\Delta t$ :

$$
C_{i}=A_{r, i-1} \frac{t_{r e t}-\frac{r_{s}}{c_{f}}}{\Delta t} \quad, \quad K_{i}=\left(1-\frac{t_{r e t}-r_{s} / c_{f}}{\Delta t}\right)
$$

If $\left(t_{r e t}-r_{s} / c_{f}\right)>\Delta t:$

$$
C_{i}=A_{r, m-1} \frac{t_{m}-\frac{r_{s}}{c_{f}}-t_{i}+t_{r e t}}{\Delta t}+A_{r, m}\left(1-\frac{t_{i}-t_{r e t}-t_{m-1}+\frac{r_{s}}{c_{f}}}{\Delta t}\right), \quad K_{i}=0
$$

In which $t_{m}$ is the time step satisfying $t_{m-1}<t_{i}-t_{r e t}+r_{s} / c_{f}<t_{m}$. 
Equation (2.13) states that the target point pressure at time $t_{i}$ has a time dependent term $C_{i}$ and a stiffness factor $K_{i}$ that do not change over time as given in equation (2.14) and (2.15). In equation (2.13) the unknown is the point source strength $A_{r, i}$. Forming a stiffness matrix by assembling the stiffness factor of every point source affecting the neighboring apex points and satisfying the boundary condition, the problem is solved for each time step. It should be mentioned that the stiffness matrix is time independent but the constant part vector changes during time steps. According to equations (2.14) and (2.15) each point source stiffness factor is non-zero over an effective area around the source therefore we have a banded sparse stiffness matrix, unlike steady state DPSM stiffness matrix which is fully populated because every point source affects all point sources of the same medium. It makes solving the transient stiffness matrix more effective. It can be solved using many pre-developed sparse matrix solvers much faster than densely populated matrix.

\section{5 t-DPSM Analysis Results}

Numerical results for the transient problem are generated using the above theory. A flat circular transducer $(4 \mathrm{~mm}$ diameter) and a point focused transducer (radius $=3$ mm, opening angle, shown in Figure $2.1,=90^{\circ}$ ) are placed inside an unbounded fluid medium (water with wave speed $1.48 \mathrm{~km} / \mathrm{sec}$ ). The time dependent transducer pressure $f(t)$ is given by

$$
f(t)=e^{-\left(t-t_{0}\right)^{2}} \sin \left(2 \pi f_{c} t\right)
$$




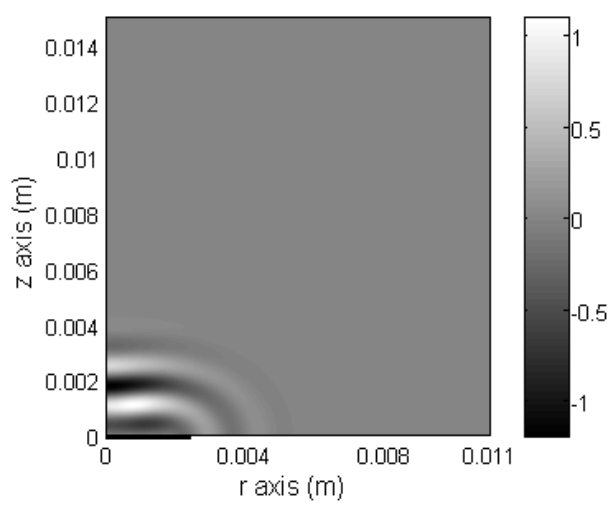

(a)

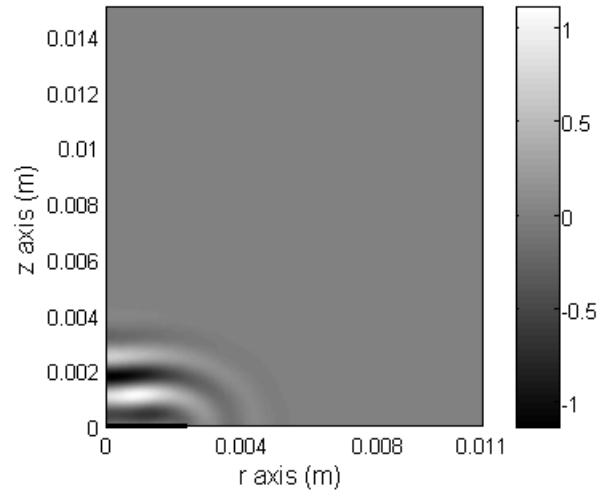

(b)

Figure 2.3 Pressure field (at $t=4 \mu \mathrm{s}$ ) generated by a flat circular transducer of diameter $4 \mathrm{~mm}$, (a): t-DPSM, (b): FEM (COMSOL). Numbers along horizontal and vertical axes are in meters. . Note that the center of the transducer is located at the origin

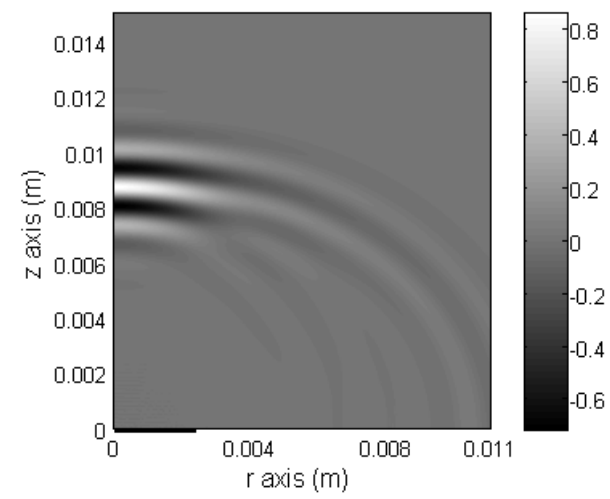

(a)

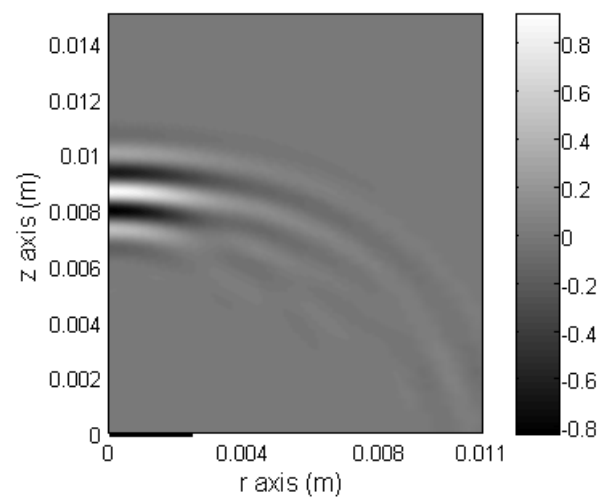

(b)

Figure 2.4 Pressure field (at $t=9 \mu \mathrm{s}$ ) generated by a flat circular transducer of diameter $4 \mathrm{~mm}$, (a): t-DPSM, (b): FEM (COMSOL). Numbers along horizontal and vertical axes are in meters. Note that the center of the transducer is located at the origin

Where $f_{c}$ is the central frequency of the signal and $t_{0}$ is the time delay. We take $f_{c}=1$ $\mathrm{MHz}$ and $t_{0}=3 \mu \mathrm{sec}$. The sampling time interval $(\Delta t)$ has been chosen as $0.15 \mu \mathrm{sec}$. The sampling frequency is $1 /(0.15 \mu \mathrm{sec})=6.67 \mathrm{MHz}$ which is 6.67 times the central 
frequency of the signal and satisfies Shannon's Sampling theorem that the sampling frequency should be at least twice the maximum significant frequency. Since the problem is linear what pressure unit is used in equation (2.16) should also be the unit for the computed pressure fields in the chapter.

In order to verify t-DPSM predictions a comparison was carried out with the FEM results that were generated using COMSOL Multi-Physics. The 2D axisymmetric transient FEM analysis was carried out. For the problem geometry with the flat circular transducer 7517 triangular elements with quadratic shape functions were considered. The focused transducer problem geometry was meshed with 6635 triangular elements with quadratic shape functions. In both models absorbing boundaries were used to prevent reflections from the artificial boundaries. Equation (2.16) was applied at the transducer boundary as the excitation force. Generalized alpha time dependent solver was chosen for simulations.

Figure 2.3 shows pressure fields in front of the flat circular transducer at $4 \mu \mathrm{sec}$ generated by t-DPSM and FEM. Figure 2.4 shows the same comparison at $9 \mu$ sec. In both figures t-DPSM shows good matching with the FEM results. Figure 2.5 compares tDPSM and FEM generated pressure time histories at an axial point $(\mathrm{r}=0.0, \mathrm{z}=4.0 \mathrm{~mm})$. Figure $2.5 \mathrm{a}$ also shows the transducer pulse from equation (2.16) with time delay $\mathrm{t}_{0}$. The wave travel time from transducer to target point is also depicted. It should be noted here that the pressure peak value at $\mathrm{z}=4 \mathrm{~mm}$ is greater than that at $\mathrm{z}=0$. 


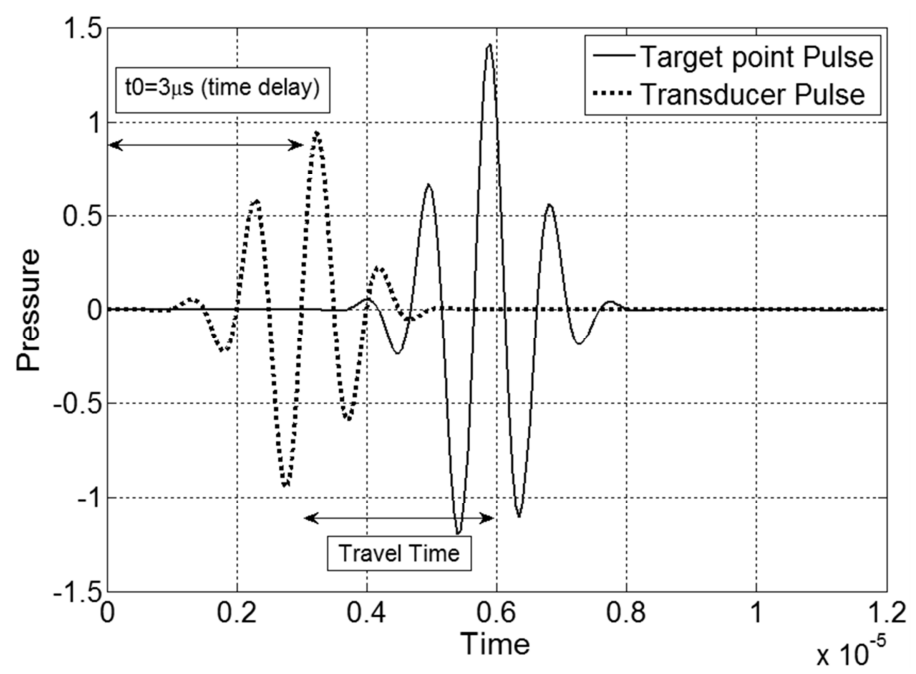

(a)

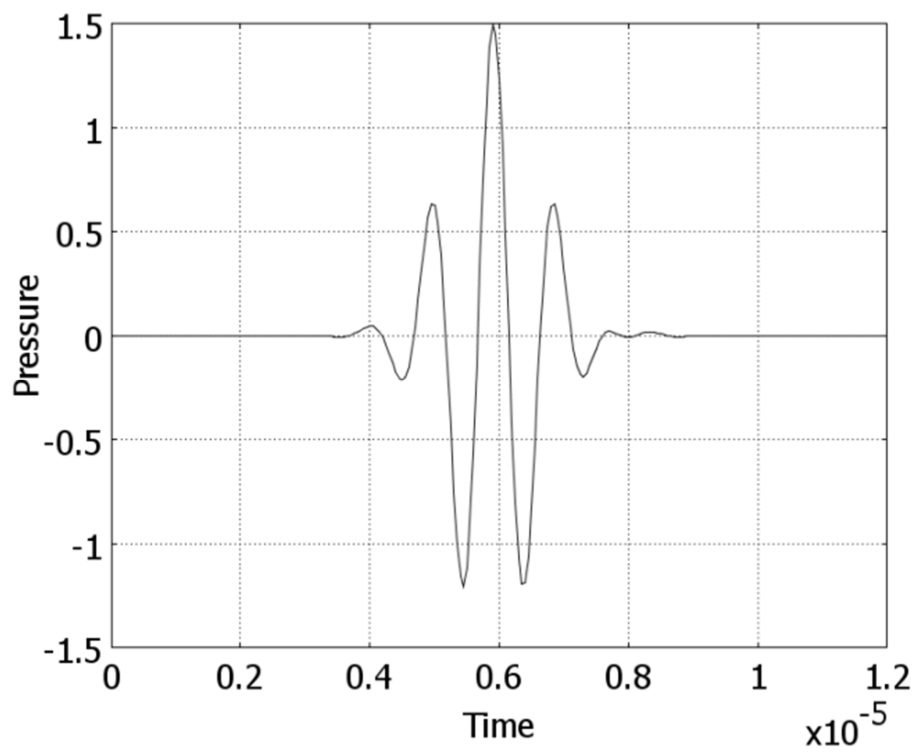

(b)

Figure 2.5 Pressure time history (at $r=0 \mathrm{~mm}, z=4 \mathrm{~mm}$ ) generated by a flat circular transducer of diameter 4 mm, (a): t-DPSM, (b): FEM (COMSOL). Time is in second, pressure units are arbitrary - Pa or psi, same as that in equation (2.16). 


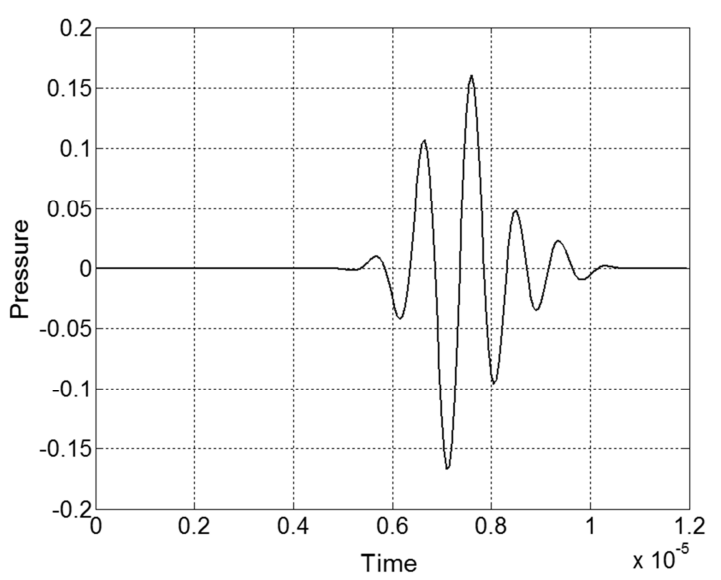

(a)

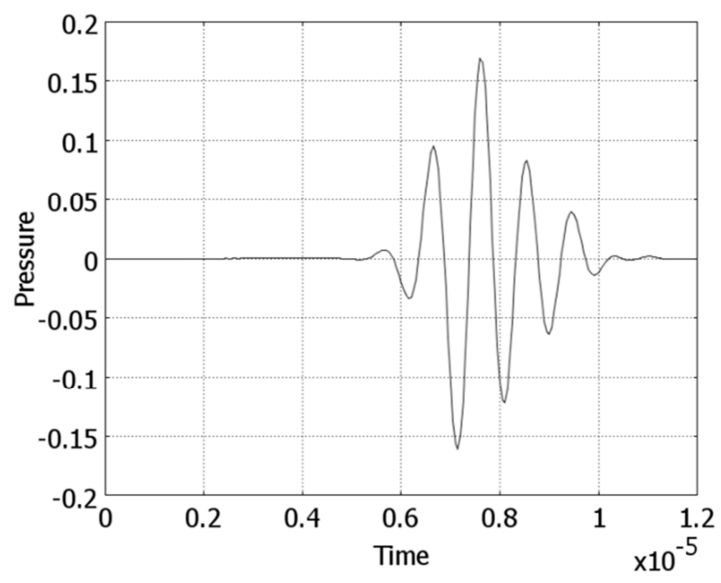

(b)

Figure 2.6 Pressure time history (at $r=4 \mathrm{~mm}, z=6 \mathrm{~mm}$ ) generated by a flat circular transducer of diameter 4 mm, (a): t-DPSM, (b): FEM (COMSOL). Time is in second, pressure units are arbitrary - Pa or psi, same as that in equation (2.16).

This observation can be justified from the fact that for the uniform pressure boundary condition on the transducer surface the largest pressure in front of the transducer appears at a point located beyond the near field distance [see Fig. 11 of Ref. [28]]. Figure 2.6 shows similar results as Figure 2.5 but at an off-axis point $(r=4.0, z=6.0 \mathrm{~mm})$. In these two figures also t-DPSM results match well with the FEM predictions. Figure 2.7 shows the pressure field in front of a focused transducer at $7 \mu$ sec generated by t-DPSM and FEM. Figure 2.8 compares t-DPSM and FEM results for the focused transducer at a point $(\mathrm{r}=0.0, \mathrm{z}=4.0 \mathrm{~mm})$ on the central axis of the transducer, again showing good matching between the two computations. 


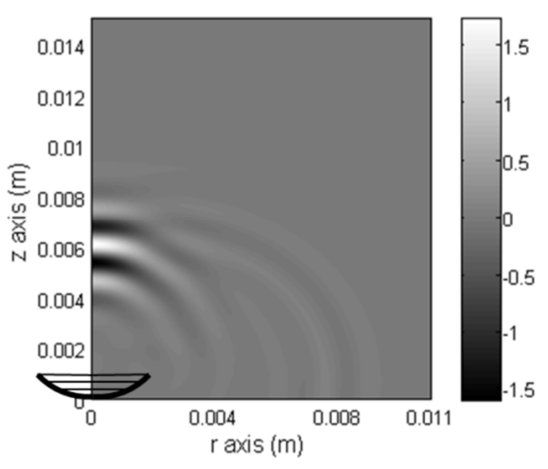

(a)

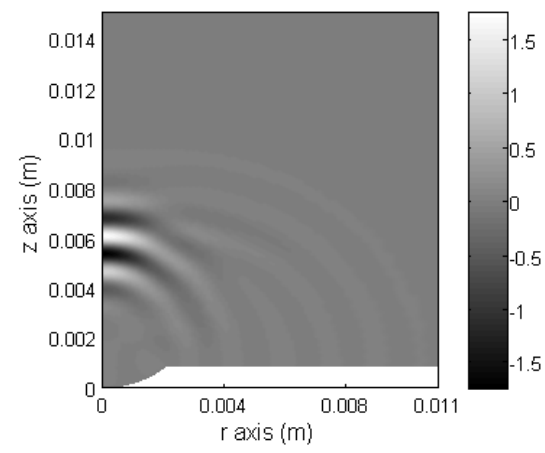

(b)

Figure 2.7 Pressure field (at $t=7 \mu \mathrm{s}$ ) generated by a point focused transducer $($ radius $=3 \mathrm{~mm}$, opening angle = 90'), (a): t-DPSM, (b): FEM (COMSOL).

The second set of results is generated by t-DPSM only, because some of these problem geometries are not axi-symmetric and therefore 2D FEM analysis cannot be applied. 3D FEM analysis takes too long to solve these problems and also suffers from the lack of accuracy (see [28]). For these computations, a spherical cavity of $0.3 \mathrm{~mm}$ diameter is placed at $3 \mathrm{~mm}$ axial distance from the transducer to investigate the scattering effect. Figure 2.9a depicts the pressure field at $6.9 \mu \mathrm{sec}$ for a cavity located at the focal point and Figure $2.9 \mathrm{~b}$ shows similar plot when the cavity is shifted horizontally by 0.5 $\mathrm{mm}$ from the focal point. Figure $2.9 \mathrm{c}$ shows the pressure field in absence of any cavity. $6.9 \mu \mathrm{sec}$ was chosen because it takes the signal reflected by the cavity approximately 6.9 $\mu$ sec after generation to reach the transducer. Comparing the white windows in three plots of Figure 2.9 one can see that, as expected more reflected waves (strong reflection) reach the transducer face when the cavity is placed at the focal point with zero eccentricity. Also no reflection is observed when there is no cavity. 


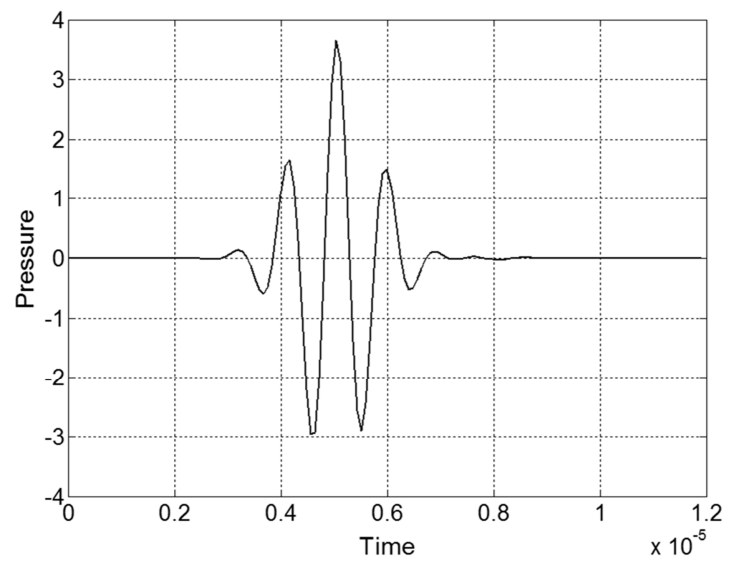

(a)

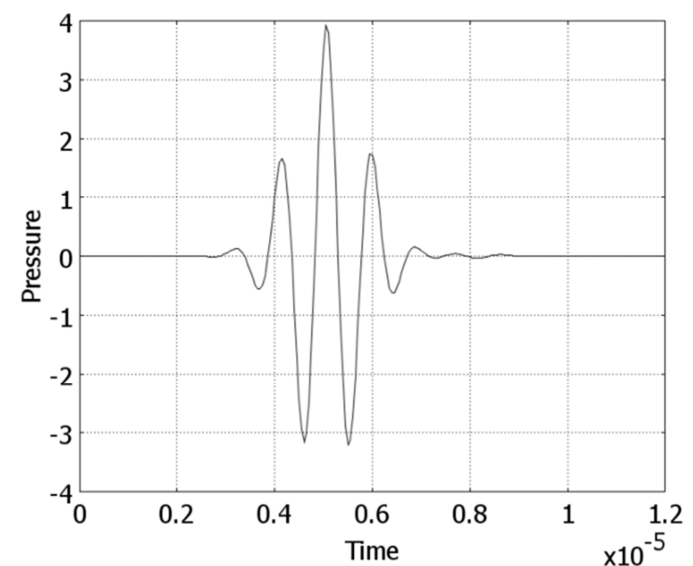

(b)

Figure 2.8 Pressure time history (at $r=0 \mathrm{~mm}, z=4 \mathrm{~mm}$ ) generated by a point focused transducer (radius $=3$ $\mathrm{mm}$, theta $\left.=90^{\circ}\right)$; (a): t-DPSM, (b): FEM $($ COMSOL). Time is in second, pressure units are arbitrary - Pa or psi, same as that in equation (2.16).

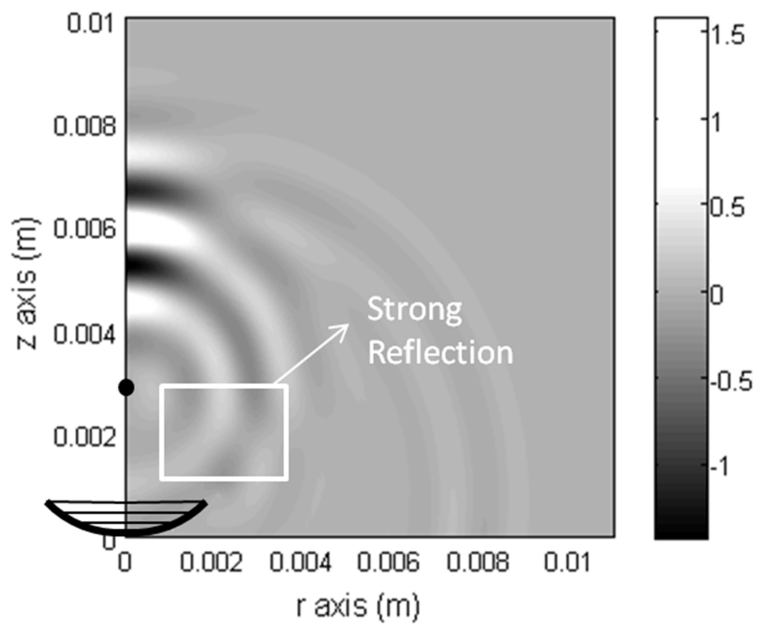

(a) 


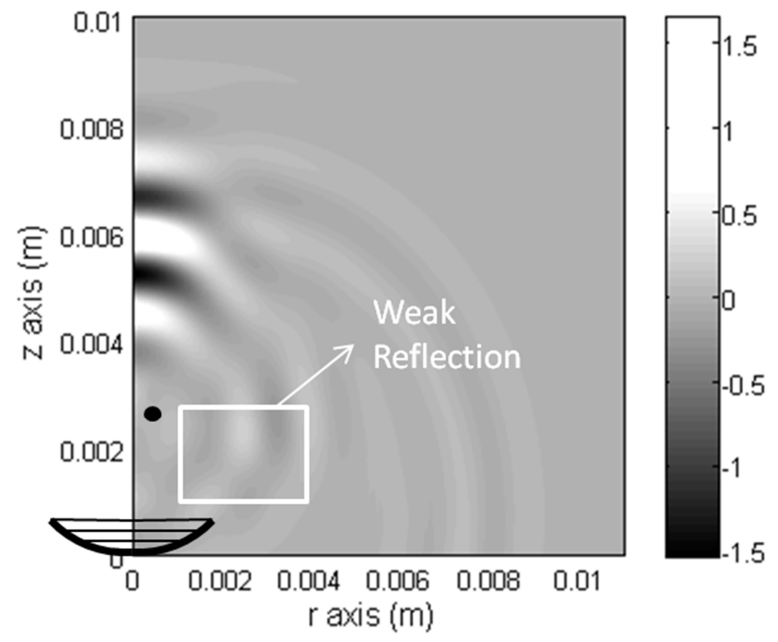

(b)

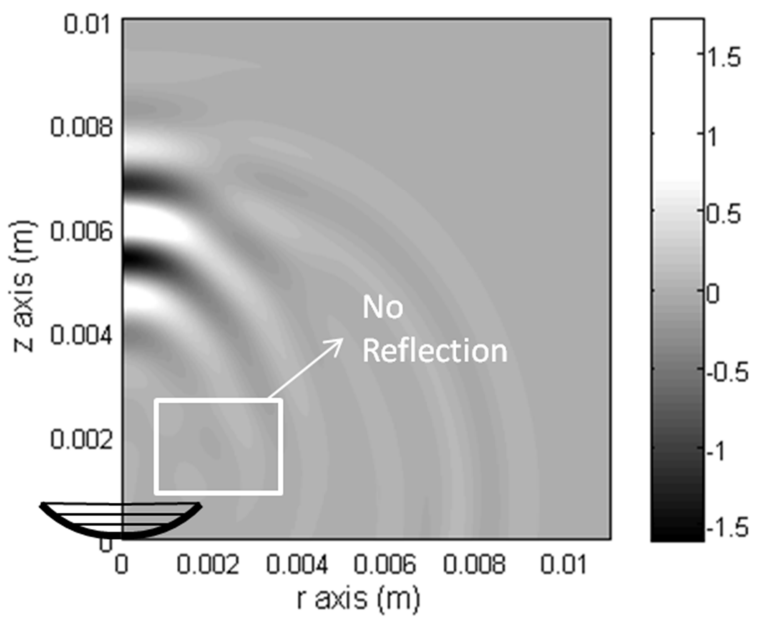

(c)

Figure 2.9 t-DPSM computed pressure fields (at $t=6.9 \mu \mathrm{s}$ ) for a point focused transducer (radius $=3 \mathrm{~mm}$, opeing angle $=\mathbf{9 0}^{\circ}$ ). Pressure fields are generated for the case with no cavity (image (c)) and in presence of a spherical cavity of diameter $0.3 \mathrm{~mm}$ with horizontal eccentricity $=0.0 \mathrm{~mm}$ (image (a)) and with horizontal eccentricity $=0.5 \mathrm{~mm}($ image $(b))$. 
Figure 2.10 shows the reflected energy which is defined as the integration of the absolute values of the pressure on the transducer surface generated by the scattered field due to the cavity. Integration of pressure is a measure of the force sensed by the transducer from the reflected wave. For uniform displacement of the transducer face this force times the uniform displacement gives a measure of the reflected energy striking the transducer surface. In order to calculate this reflected energy or force only the cavity point source contributions need to be taken into account. This integral value of the pressure field gives the total strength or energy of the reflected signal from the cavity. Energy variations for three different eccentricities are compared -1) when the cavity is located at the focal point (zero eccentricity), 2) shifted horizontally by $0.3 \mathrm{~mm}$ and 3) by $0.5 \mathrm{~mm}$. From these curves the time of arrival of the reflected signal at the transducer position is found around $7 \mu \mathrm{sec}$. From equation (2.16) one can see that the transducer signal peak occurs at $3 \mu \mathrm{sec}$ therefore the difference $7-3=4 \mu \mathrm{sec}$ is the required travel time for the wave departing from the transducer, hitting the cavity, and then returning to the transducer. Considering the wave speed value in water one gets the travel time $2 * 0.003 \mathrm{~m} /(1480 \mathrm{~m} / \mathrm{s}) \approx 4 \mu \mathrm{sec}$ which matches well with t-DPSM results. The second peak near $11 \mu \mathrm{sec}$ verifies an additional $4 \mu \mathrm{sec}$ travel time for the second order reflection. These curves also show that the received signal strength decreases when the cavity is shifted horizontally. 


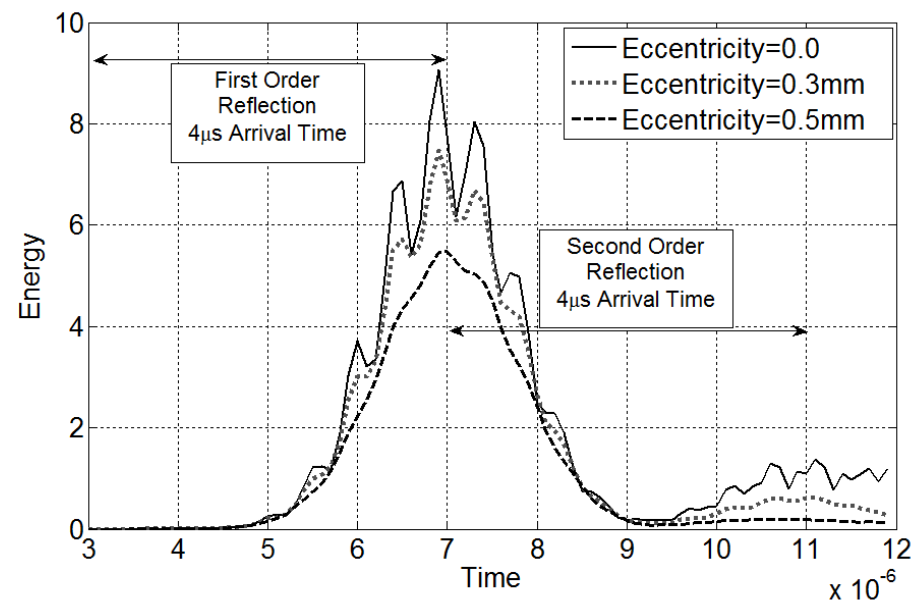

Figure 2.10 Reflected signal strength or energy (from a spherical cavity of $0.3 \mathrm{~mm}$ diameter placed at the focal plane) as a function of time for a point focused transducer (radius $=3 \mathrm{~mm}$, opening angle $=90^{\circ}$ ) for different horizontal eccentricities. Time is in second, the energy has arbitrary unit that depends on the unit of equation

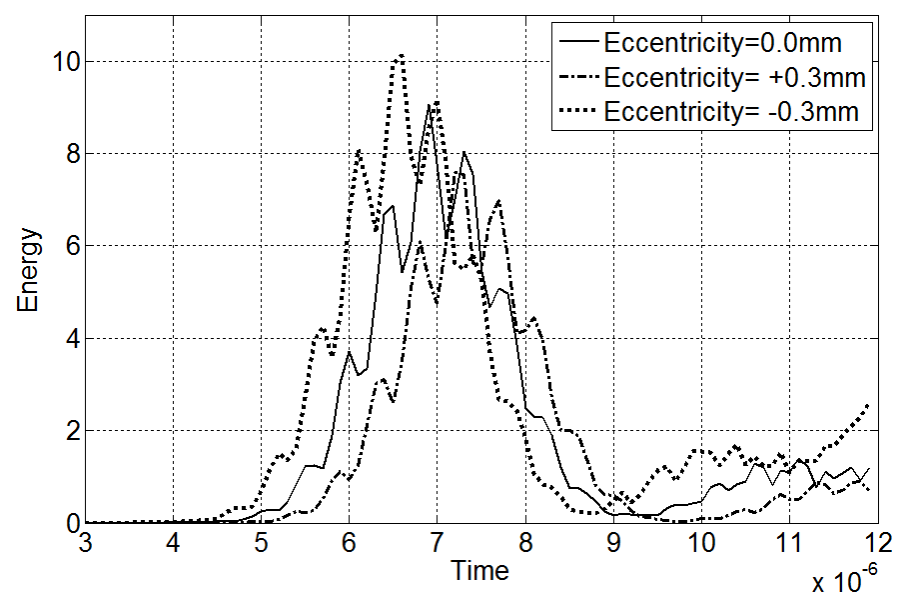

Figure 2.11 Reflected signal strength or energy (from a spherical cavity of $0.3 \mathrm{~mm}$ diameter placed on the transducer axis of symmetry) as a function of time for a point focused transducer (radius $=3 \mathbf{m m}$, opening angle $=90^{\circ}$ ) for three different locations of the cavity - cavity eccentricities are zero (cavity is placed at the focal point), $\mathbf{- 0 . 3} \mathrm{mm}$ (cavity is moved from the focal point by $0.3 \mathrm{~mm}$ towards the transducer) and $+0.3 \mathrm{~mm}$ (moved away from the transducer). Time is in second, the energy has arbitrary unit that depends on the unit of equation (2.16). 


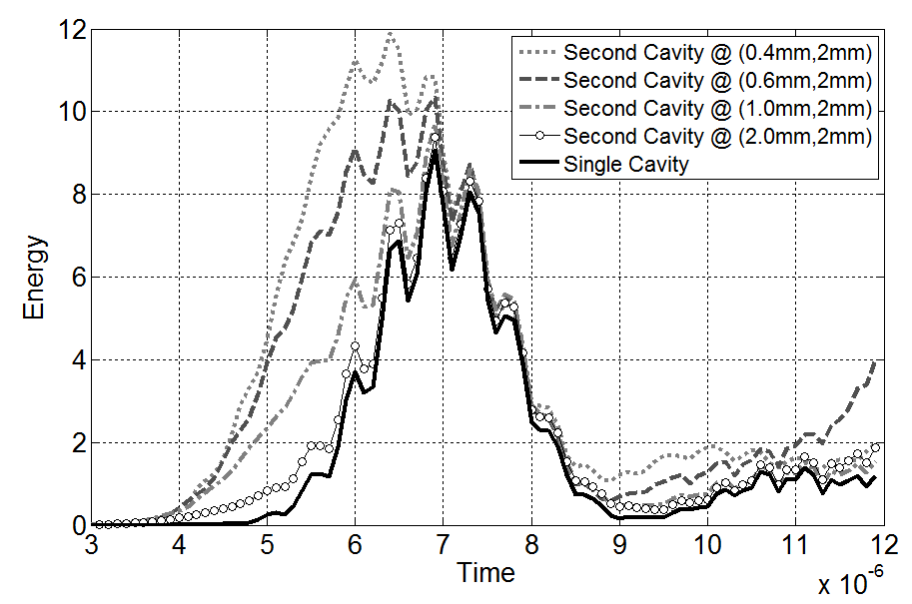

Figure 2.12 Reflected signal strength or energy (from two spherical cavities of $0.3 \mathrm{~mm}$ diameter) as a function of time for a point focused transducer (radius $=3 \mathrm{~mm}$, opening angle $=90^{\circ}$ ), one cavity is located at the focal point and the second cavity is placed at four different locations in $(r, z)$ coordinates. Time is in second, the energy has arbitrary unit that depends on the unit of equation (2.16).

Figure 2.11 shows same type of curves as in Figure 2.10 when the cavity is placed first at the focal point and then shifted axially up or down by $0.3 \mathrm{~mm}$. The signal peak decreases when the cavity moves away from the transducer and increases when it comes closer. In these curves one can clearly see that the arrival times of the reflected signal peaks are shifted as the cavity position is moved axially. For $0.3 \mathrm{~mm}$ eccentricity the arrival time shift should be theoretically $2 * 0.3 \mathrm{~mm} /(1480 \mathrm{~m} / \mathrm{s}) \approx 0.4 \mu \mathrm{sec}$ that matches with the model predictions.

Figure 2.12 shows the reflected energy curves for double cavities when the first cavity is placed at the focal point and the second one is placed at $2 \mathrm{~mm}$ vertical distance from the transducer and shifted axially by $+0.4 \mathrm{~mm},+0.6 \mathrm{~mm},+1 \mathrm{~mm}$ and $+2 \mathrm{~mm}$. The Energy signals are compared with the signal generated by a single cavity placed at the focal point. As expected, two cavities in close proximity generate stronger reflected 
energy. As the second cavity moves away from the first cavity the reflected signal becomes closer to the single cavity curve; in other words, the effect of the second cavity becomes negligible as it moves away.

\subsection{Summary}

In this chapter a transient Green's function based method following the concepts of conventional DPSM is presented. The transient DPSM method or t-DPSM offers a significant advantage over the conventional numerical methods like FEM. Advantages of DPSM over FEM and BEM for solving ultrasonic wave propagation problems were discussed above and established earlier in other publications on DPSM. The main advantage of DPSM is that computationally it is much faster than FEM for threedimensional large domain problems because DPSM is a mesh-free technique. t-DPSM that follows the same concepts has similar advantages. t-DPSM generated results matched well with the FEM predictions for some sample problems. For more complex non-axisymmetric problems for which 3D FEM analysis is too time consuming t-DPSM is easy to implement. Unlike the steady state DPSM the stiffness matrix generated in tDPSM is sparsely populated and banded, also it is constant over time that makes t-DPSM computationally very efficient. Some applications of t-DPSM for ultrasonic field modeling for focused and flat circular transducers in presence and absence of spherical cavities in a fluid have been presented in this chapter. It should be noted here that $\mathrm{t}$ DPSM does not require FFT inversion for generating the transient signal. The conventional FFT based technique is good for generating complete time histories from the frequency spectra that decay fast. If the frequency spectrum decays slowly then one 
needs to compute the steady state response at many frequencies even when one is interested in studying only a small part of the complete time history. In that situation the t-DPSM technique should be the method of choice

\subsection{Future Works}

So far t-DPSM has been investigated to model ultrasonic wave propagation in fluid media. The proposed method can be extended to solid medium and problem geometries involving fluid and solid media in the future. The current t-DPSM method is only capable of modeling linear problems. Therefore, there is a need to investigate how t-DPSM can be extended to non-linear problems as well. 


\section{CHAPTER 3}

\section{MODIFICATIONS OF DPSM FOR ULTRASONIC AND}

\section{ELECTROMAGNETIC FIELD MODELING PROBLEMS}

\subsection{INTRODUCTION}

The basic principle of DPSM has been discussed above and described in detail in Refs. [26, 27, 41, 64]. The interaction between ultrasonic waves and fluid-solid interfaces was studied in Refs. [31, 63]. Ultrasonic field modeling in multilayered fluid structures using DPSM technique was reported by Banerjee et al. [29]. Scattering of ultrasonic waves due to spherical and elliptical cavities has been investigated in Refs. [32, 49, 66]. The interaction between bounded ultrasonic beams and corrugated plates has been also studied $[42,43]$. Electromagnetic acoustic transducers have been modeled using DPSM by Eskandarzade et al. [67]. Kundu et al. [28] compared FEM and DPSM techniques for modeling circular and rectangular transducers. They showed that FEM-3D at high frequencies suffers from the lack of accuracy unless the domain is meshed with very small elements that makes this method computationally very time consuming and impossible to handle by many computers while the DPSM technique is much faster and it produces results that matches very well with the analytical solutions.

The main shortcoming of DPSM is that when the boundary conditions are satisfied only at the apex points of the spheres (surrounding the point sources) where they touch the transducer surface the computed field needs to be scaled up to match with the theoretical solution. This is because the velocity values generated by the point sources are highest at 
the apex points, therefore the average value of the transducer velocity comes out to be less than the prescribed value if the boundary conditions are satisfied only at the apex points. So far there have been two suggestions to avoid this scaling need -1 ) satisfy the boundary conditions at boundary points that are randomly distributed around the apex points, as done in some publications on DPSM and 2) satisfy boundary conditions on and beyond the transducer face at more points than the number of unknowns and thus solve an over-determined system by least-squares error minimization technique as done by Cheng et al. [65]. The problem with option 1 is that random selection of boundary points are not always very reliable and sometimes a mismatch between the DPSM results and the theoretical results are observed even after random selection of target points; only after scaling this mismatch can be completely eliminated. The problem with the second option is that it requires solution of an over-determined system and therefore requires more computational effort.

In this chapter two new modifications are proposed to bypass the scaling needs. In the first modification which will be called Gaussian DPSM (or G-DPSM) one point source is replaced by multiple fictitious or child point sources whose strengths are governed by the Gaussian distribution functions. The computed field by G-DPSM without any scaling matches well with the analytical solution. The second method called the Element Source Method (ESM) is then introduced. Point sources in DPSM are replaced by element sources in ESM. It should be noted that in ESM the system of equations are not derived from the boundary integral equations as done in the boundary 
element method BEM (see [68]). Advantages and disadvantages of G-DPSM and ESM are also discussed.

Modeling ultrasonic fields in front of a transducer is a fundamental problem that has been attempted by different methods based on analytical, semi-analytical and numerical techniques (see [69-72]). G-DPSM and ESM methods are illustrated in this chapter by computing the pressure field in front of circular and rectangular ultrasonic transducers and comparing the computed results with the available analytical solutions.

Modeling electromagnetic Gaussian beam in front of an emitter is a fundamental problem that has been studied in section 1.7.3 using DPSM. It is shown that by increasing the source radius the DPSM solution does not converges to analytical one but to the scaled form of the analytical curve which will be shown as the chapter is followed. ESM methods are illustrated by computing the electric field in front of circular Gaussian beam emitter and comparing the computed results with the available analytical solutions.

\subsection{Scaling needs in the conventional DPSM}

In DPSM the target points of Figure 3.1 are placed very close to the transducer face [4] or on the transducer face then the generated ultrasonic field at the target points shows some non-uniformity even when uniform point source strengths are considered. Figure 3.2 shows the ultrasonic field (velocity normal to the transducer face) on a circular transducer face generated by 499 point sources modeling the transducer face. To generate Figure 3.2 the number of target points on the transducer face is taken many more than 500 to compute fields in between the peak points as well. Although the computed 
normal velocity at the 499 points where the small spheres touch the transducer face is same (equal to 1) it is lower in between these points.

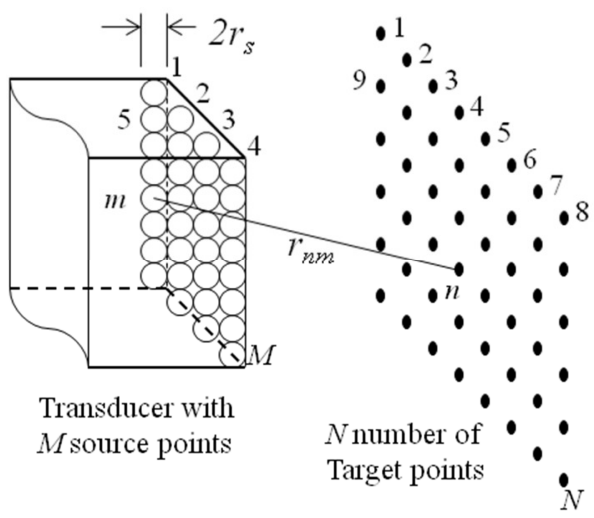

Figure 3.1 Ultrasonic field modeling by DPSM - superimposing the contributions of M source points distributed over the transducer face the ultrasonic field at $\mathbf{N}$ target points can be obtained [28].

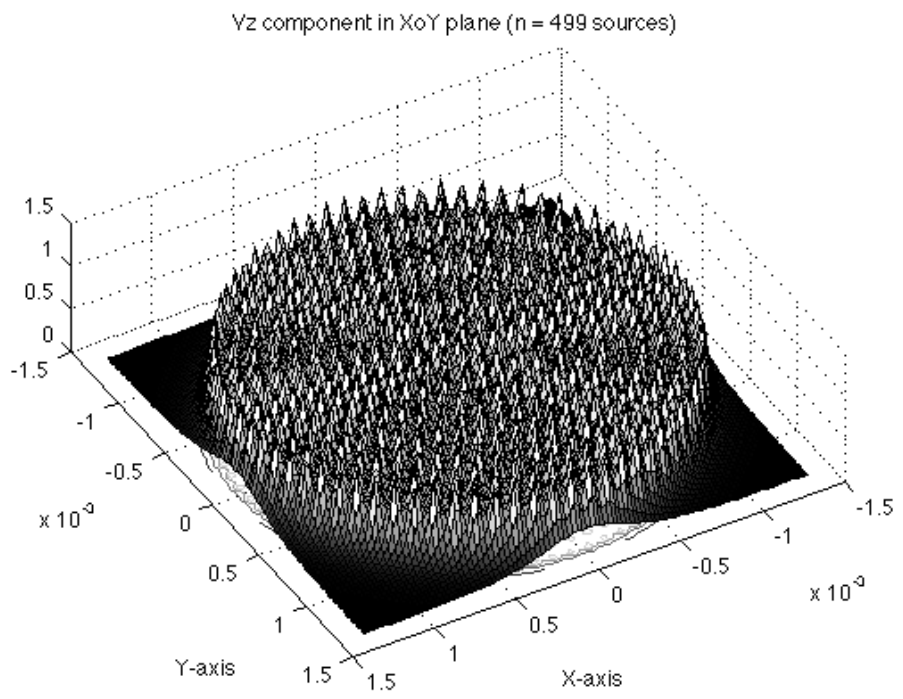

Figure 3.2 Variation of the normal velocity on the transducer surface obtained from the conventional DPSM analysis. Peak value of 1 is obtained at 499 points on the transducer surface where small spheres (see Figure 3.1) corresponding to 499 point sources touch the transducer face. Lower velocity values in between these peaks make the average normal velocity of the transducer surface less than 1 [see [28]]. 


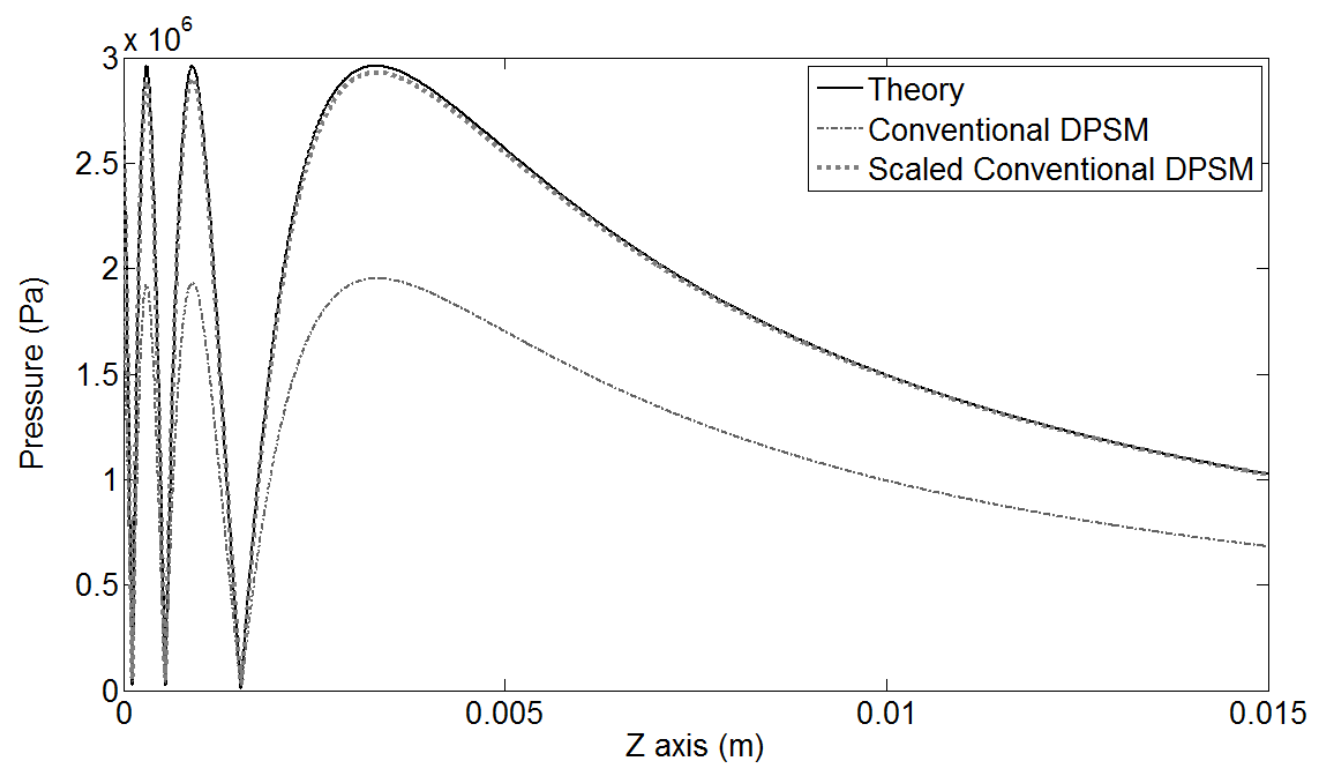

Figure 3.3 Comparison between theoretical and DPSM (with and without scaling) generated pressure fields along the central axis of a circular transducer of $2 \mathrm{~mm}$ diameter vibrating at $5 \mathrm{MHz}$ frequency

Therefore, the average velocity of the transducer face is smaller than what is computed at these 499 points. To compute the point source strengths if we only equate the transducer surface velocity at these 499 points to the specified velocity (say 1), since the average transducer surface velocity is less than 1 the computed ultrasonic field should be smaller than the true values as shown in Figure 3.3. The theoretical curve shown as the continuous line is obtained from the Rayleigh integral, also known as the RayleighSommerfeld integral (RSI) [26, 27, 41, 64]. Two DPSM generated curves are shown by dashed and dotted lines. Dashed line is from the conventional DPSM analysis without any scaling while the dotted line is obtained after scaling the results so that the average velocity of the transducer surface becomes 1. After scaling, the DPSM prediction matches well with the theoretical curve. 


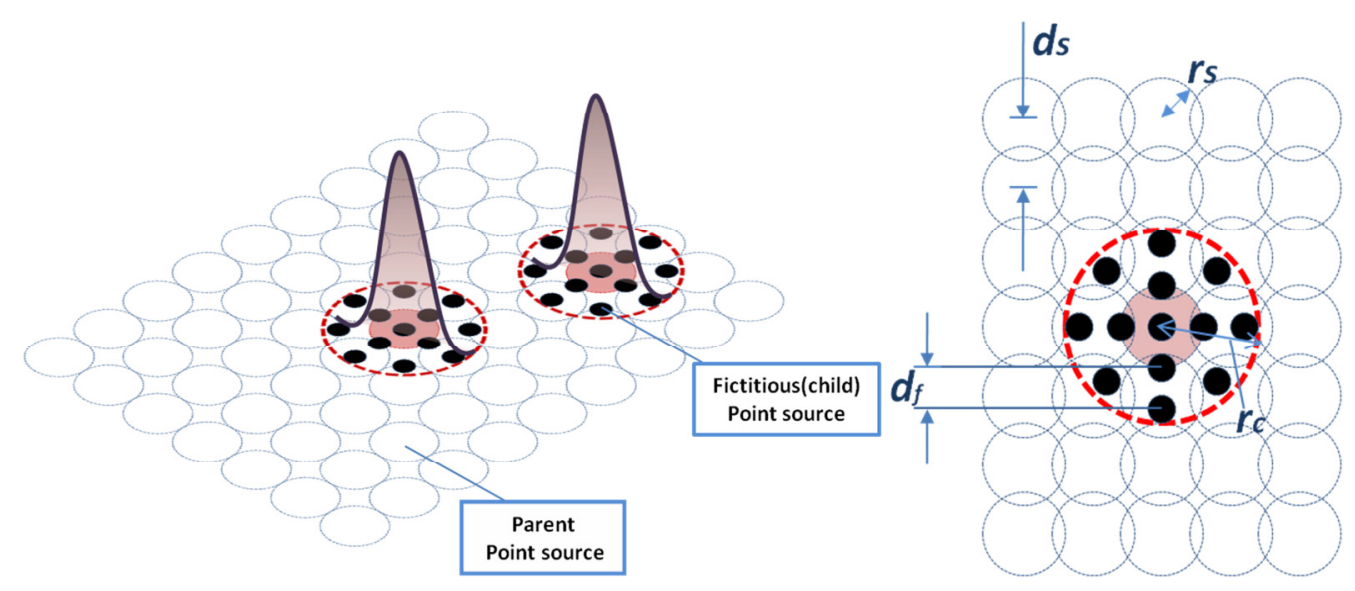

Figure 3.4 Schematic depiction of Parent and Fictitious (child) Gaussian Point sources

For generating the curves of Figure 3.3, the point source strengths are computed assuming uniform unit normal velocity at the target points placed on the transducer surface. The analytical solution for the pressure distribution along the central axis is obtained from the Rayleigh-Sommerfeld Integral of type II. For a circular transducer face vibrating with uniform unit normal velocity the pressure field in front of the transducer is given by [73],

$$
p(z)=\rho c_{f} v_{0}\left[\exp \left(i k_{f} z\right)-\exp \left(i k_{f} \sqrt{z^{2}+R^{2}}\right)\right]
$$

In equation (3.1) $\rho$ is the fluid density, $c_{f}$ is the P-wave speed in the fluid, $v_{0}$ is the transducer surface velocity, $z$ is the distance of the point from the transducer face, $k_{f}(=$ $\omega / c_{f}$ ) is the wave number in the fluid, $\omega$ is the angular frequency of the signal, and ' $R$ ' is the radius of the transducer. 


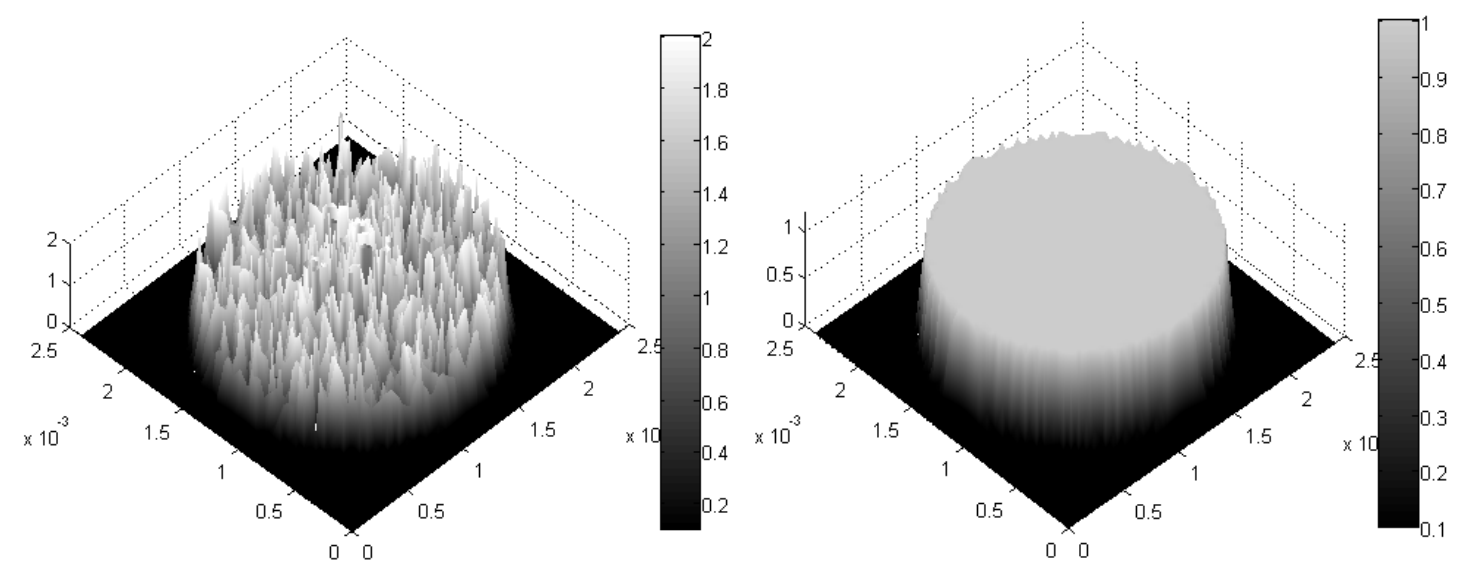

(a)

(b)

Figure 3.5 Velocity distribution on the transducer surface, Gaussian DPSM, 2 mm diameter transducer, Frequency $=5$ MHz: (a) when $a=r_{s}(b)$ when $a=0.5 r_{s}$

\subsection{Proposed Gaussian-DPSM (G-DPSM) method}

In the following, a modification to the conventional DPSM is presented. In the proposed new method point sources (Parent sources) are accompanied by a group of fictitious point sources (or child sources) surrounding every parent source in an effective domain as depicted in Figure 3.4. It is assumed that the strength of every child source is a function of its distance from its parent source as shown below:

$$
A_{s i}=A_{s} * f\left(r_{i}\right) \text { and } f(r)=\exp \left(\left(-\left(\frac{r}{a}\right)^{2}\right)\right.
$$

In which $A_{s i}$ is the strength of the $i$-th fictitious point source at a distance $r_{i}$ from the parent source and $A_{s}$ is the strength of the parent source. ' $a$ ' is a controlling parameter changing the bell shape width of the Gaussian function. It should be noted that in spite of using the fictitious point sources the number of equations to be solved is not changed 
because the boundary conditions are satisfied corresponding to the parent source points. As depicted in Figure 3.4 the child (fictitious Gaussian) point sources are distributed over a circular domain with radius 1.1 times the average distance between the neighboring parent point sources $\left(r_{c}=1.1 d_{s}\right.$, see Figure 3.4) The average distance between the Gaussian child sources is set equal to $5 \%$ of the circle radius $\left(d_{f}=0.05 r_{c}\right.$, see Figure 3.4).

The Gaussian-DPSM analysis has been carried out for two values of the controlling parameter $a ; a=0.5 r_{s}$ and $a=r_{s}$ in which $r_{s}$ is the radius of the sphere surrounding the parent point source. Figure 3.5a and Figure 3.5b show the velocity distribution on the transducer surface obtained from G-DPSM when $a=r_{s}$ and $a=0.5 r_{s}$, respectively. Clearly using a smaller ' $a$ ' value results in a more uniform and smooth distribution of the transducer surface velocity. Figure 3.6 plots the central axis pressure field for the $2 \mathrm{~mm}$ diameter transducer vibrating at $5 \mathrm{MHz}$ frequency. G-DPSM $\left(a=0.5 r_{s}\right)$ predicted results show good agreement with the theoretical results.

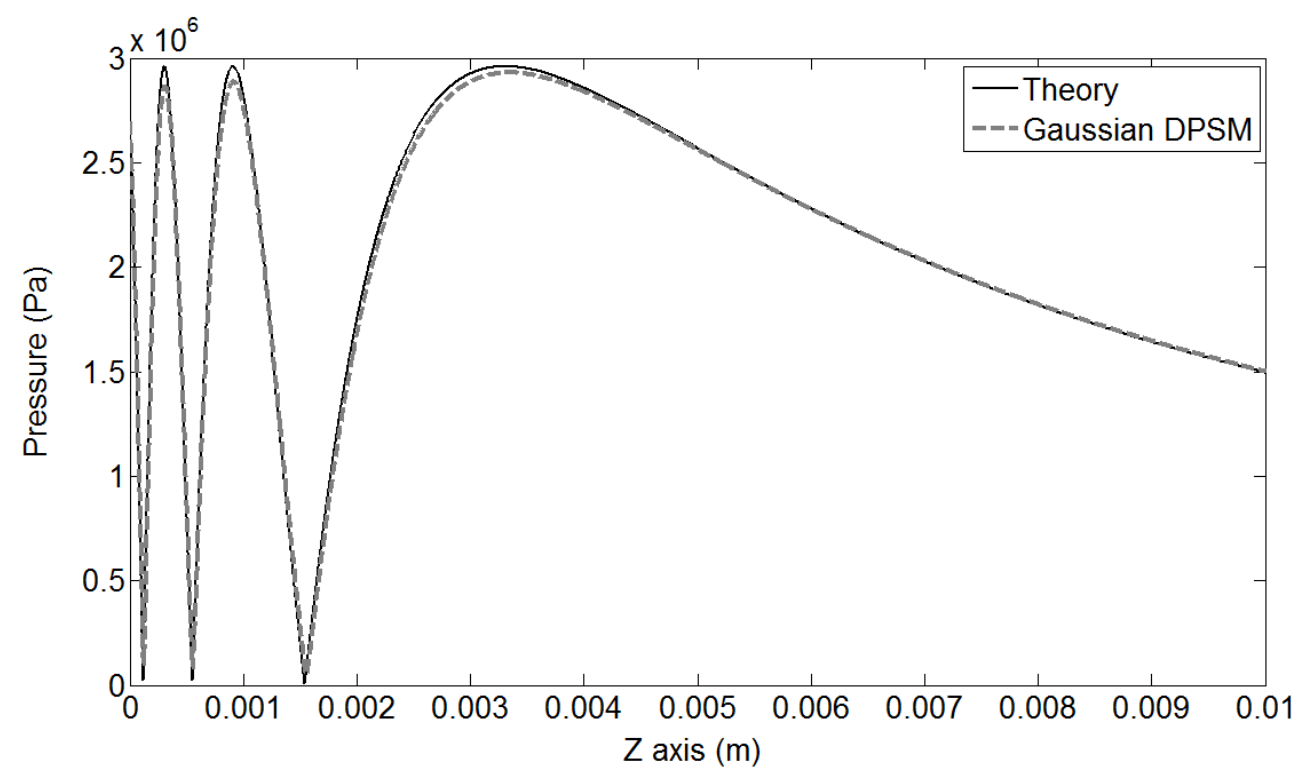

Figure 3.6 Comparison between theoretical and Gaussian DPSM $\left(a=0.5 r_{s}\right)$, generated pressure fields on the central axis of a circular transducer of $2 \mathrm{~mm}$ diameter vibrating at $5 \mathrm{MHz}$ frequency 


\subsection{Proposed Element Source Method (ESM) in Ultrasonic}

G-DPSM has two drawbacks - 1) it is very sensitive to the controlling parameter ' $a$ ' as demonstrated in Figure 3.5 and 2) considering a group of child point sources for every parent source makes the technique computationally slow even though the total number of unknowns and the number of equations to be solved are not affected by the number of additional fictitious sources. Following the main concept of Gaussian DPSM that places additional sources in between discrete point sources the Element Source Method (ESM) is developed. In this method instead of using only discrete point sources to model the interfaces and boundaries it is assumed that the source strength continuously varies between the point sources. Therefore, in the ESM modeling after placing the point sources along the boundary the source strength is continuously varied between these point sources. Unlike DPSM where no other form of source exists between two neighboring point sources in ESM continuous source exists between the point sources.

As shown in Figure 3.7(a) if $A_{S 1}, A_{S 2}, A_{S 3}$ and $A_{S 4}$ are source strengths at 4 nodes of a linear element then the source strength within the element can be expressed in terms of the local coordinates and linear shape functions or interpolation functions as used in finite element and boundary element methods:

$$
A_{s}(\xi, \eta)=\sum_{i=1}^{n} A_{S i} N_{i}(\xi, \eta)
$$

$n=$ number of element nodes $(n=4$ for a linear element $)$ 


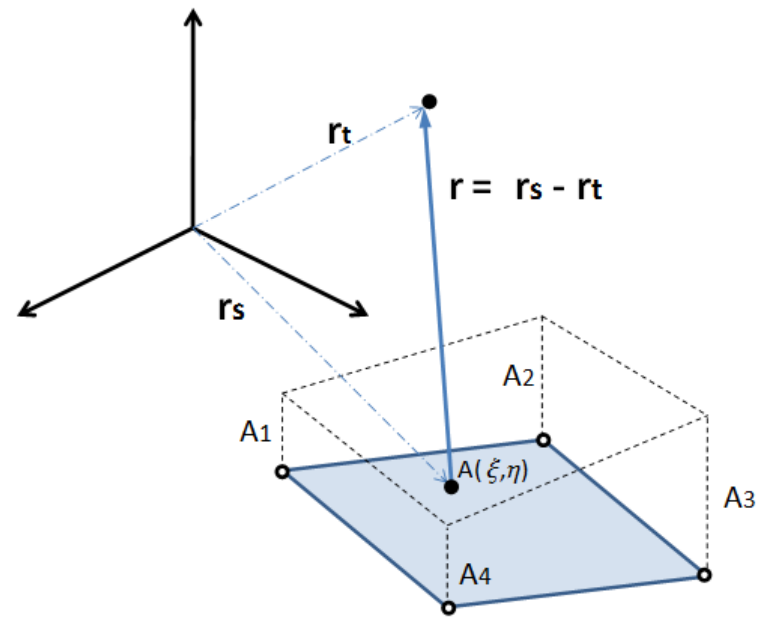

(a)
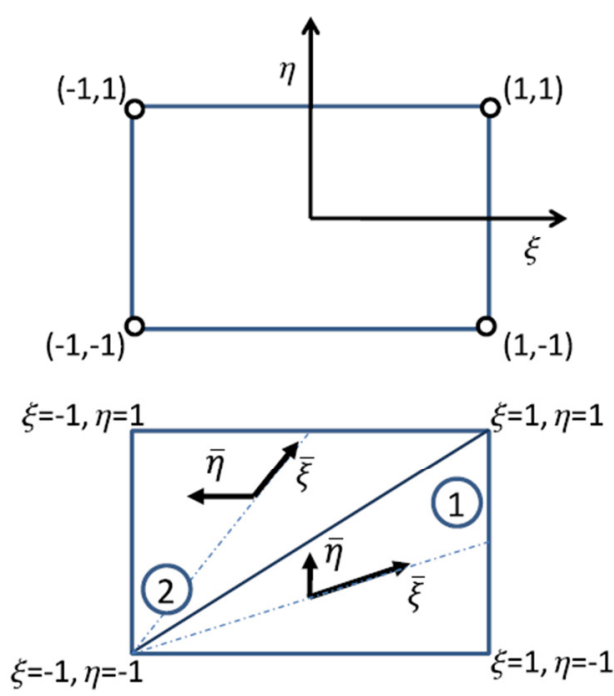

(b)

Figure 3.7 (a) Schematic depiction of a source element in global coordinate (b) Main element and the subelements in local coordinate

$$
\begin{aligned}
& N_{1}=\frac{1}{4}(1+\xi)(1+\eta) \\
& N_{2}=\frac{1}{4}(1-\xi)(1+\eta) \\
& N_{3}=\frac{1}{4}(1-\xi)(1-\eta) \\
& N_{4}=\frac{1}{4}(1+\xi)(1-\eta)
\end{aligned}
$$

Quadratic shape functions should be used for quadric (8 node) elements. The unknown parameters in ESM formulation are the source strengths at the element nodes. For linear and quadric elements 4 and 8 nodes of the element represent 4 and 8 point sources, respectively with unknown strengths. Since the source strength is defined continuously using the continuous interpolation functions, in the ESM formulation the compatibility and boundary conditions can be satisfied at non-nodal points as well.

The position vector of a point in the element with $(\xi, \eta)$ coordinate is given by: 


$$
\mathbf{r}_{s}(\xi, \eta)=\sum_{i=1}^{4} \mathbf{r}_{s i} N_{i}(\xi, \eta)
$$

The pressure and velocity at a target point is computed by integrating the corresponding Green's functions over the element:

$$
\begin{gathered}
P_{e}\left(\mathbf{r}_{\mathbf{t}}\right)=\int A_{s} \frac{\exp \left(i k_{f} r\right)}{r} d s \\
\mathbf{V}_{e}\left(\mathbf{r}_{\mathbf{t}}\right)=\int A_{s} \frac{\left(\mathbf{r}_{\mathrm{s}}-\mathbf{r}_{\mathbf{t}}\right)}{i \omega \rho} \frac{\exp \left(i k_{f} r\right)}{r^{2}}\left(i k_{f}-\frac{1}{r}\right) d s
\end{gathered}
$$

In which $r=\left|\mathbf{r}_{\mathbf{t}}-\mathbf{r}_{\mathbf{s}}\right|$ is the distance between the source point and the target point.

$$
\begin{gathered}
\mathbf{V}_{e}\left(\mathbf{r}_{\mathbf{t}}\right)=\sum_{i=1}^{n} A_{S i} \iint N_{i}(\xi, \eta) \frac{\left(\mathbf{r}_{\mathbf{s}}-\mathbf{r}_{\mathbf{t}}\right)}{i \omega \rho} \frac{\exp \left(i k_{f} r\right)}{r^{2}}\left(i k_{f}-\frac{1}{r}\right) d s \\
\mathbf{V}_{e}\left(\mathbf{r}_{\mathbf{t}}\right)=\sum_{i=1}^{n} A_{S i} \mathbf{V}_{e i}\left(\mathbf{r}_{\mathbf{t}}\right)
\end{gathered}
$$

In which:

$$
\mathbf{V}_{e i}\left(\mathbf{r}_{\mathbf{t}}\right)=\iint N_{i}(\xi, \eta) \frac{\left(\mathbf{r}_{\mathbf{s}}-\mathbf{r}_{\mathbf{t}}\right)}{i \omega \rho} \frac{\exp \left(i k_{f} r\right)}{r^{2}}\left(i k_{f}-\frac{1}{r}\right) d s
$$

In order to be able to carry out the integration over the element the local coordinate and Jacobian transformation matrix are implemented:

$$
\mathbf{V}_{e i}\left(\mathbf{r}_{\mathbf{t}}\right)=\iint N_{i}(\xi, \eta) \frac{\left(\mathbf{r}_{\mathrm{s}}-\mathbf{r}_{\mathbf{t}}\right)}{i \omega \rho} \frac{\exp \left(i k_{f} r\right)}{r^{2}}\left(i k_{f}-\frac{1}{r}\right) J(\xi, \eta) d \xi d \eta
$$

In which: 


$$
\mathbf{V}=\left[\begin{array}{l}
\frac{\partial y}{\partial \xi} \frac{\partial z}{\partial \eta}-\frac{\partial y}{\partial \eta} \frac{\partial z}{\partial \xi} \\
\frac{\partial z}{\partial \xi} \frac{\partial x}{\partial \eta}-\frac{\partial z}{\partial \eta} \frac{\partial x}{\partial \xi} \\
\frac{\partial x}{\partial \xi} \frac{\partial y}{\partial \eta}-\frac{\partial x}{\partial \eta} \frac{\partial y}{\partial \xi}
\end{array}\right] \quad, \quad J(\xi, \eta)=|\mathbf{V}|
$$

The integration in equation (3.11) has no closed form solution so Gaussian quadrature numerical Integration technique is used.

The integrand in equation (3.11) is singular (when $r=0$ ). To avoid this singularity the source points are shifted by an amount " $r_{S}$ " (see [26]) from the boundary as shown in Figure 3.1. After the source shifting, in order to get more accurate numerical integration results the element is divided into two sub-elements and a local coordinate system is introduced. The local coordinate system is chosen in such a way that the Jacobian of the transformation approaches zero at element nodes (see Figure 3.7(b)). This method gives a more uniform integrand within the element and finally more accurate integration value. This approach is widely used in boundary element method to solve the singularity problem during integration (see [pages 157-160 of Ref. [74]]); finally, integrating over the two sub elements we get:

$$
\mathbf{V}_{e i}\left(\mathbf{r}_{\mathbf{t}}\right)=\sum_{n=1}^{2} \int_{-1}^{1} \int_{-1}^{1} N_{i}(\xi, \eta) \frac{\left(\mathbf{r}_{\mathrm{s}}-\mathbf{r}_{\mathbf{t}}\right)}{i \omega \rho} \frac{\exp \left(i k_{f} r\right)}{r^{2}}\left(i k_{f}-\frac{1}{r}\right) J(\xi, \eta) \bar{J}(\bar{\xi}, \bar{\eta}) d \bar{\xi} d \bar{\eta}
$$

In which the shape function converting the sub-element local coordinate $(\bar{\xi}, \bar{\eta})$ to the main element local coordinate $(\xi, \eta)$ reads like: 


$$
\xi=\sum_{i=1}^{3} \xi_{i} \bar{N}_{i}(\bar{\xi}, \bar{\eta})
$$

In which:

$$
\begin{gathered}
\bar{N}_{1}=\frac{1}{4}(1+\bar{\xi})(1-\bar{\eta}) \\
\bar{N}_{2}=\frac{1}{4}(1+\bar{\xi})(1+\bar{\eta}) \\
\bar{N}_{3}=\frac{1}{2}(1-\bar{\xi})
\end{gathered}
$$

Also the Jacobian transformation from Element to Sub-element coordinate is:

$$
\bar{J}=\left|\begin{array}{ll}
\frac{\partial \xi}{\partial \bar{\xi}} & \frac{\partial \eta}{\partial \bar{\xi}} \\
\frac{\partial \xi}{\partial \bar{\eta}} & \frac{\partial \eta}{\partial \bar{\eta}}
\end{array}\right|
$$

Finally the Gaussian quadrature integration form of equation (3.13) gives:

$$
\begin{aligned}
& \mathbf{V}_{e i}\left(\mathbf{r}_{\mathbf{t}}\right)=\sum_{n=1}^{2} \sum_{m=1}^{n p x} \sum_{k=1}^{n p y} N_{i}\left(\bar{\xi}_{m}, \bar{\eta}_{k}\right) \frac{\left(\mathbf{r}_{\mathrm{s}}-\mathbf{r}_{\mathbf{t}}\right)}{i \omega \rho} \frac{\exp \left(i k_{f} r\right)}{r^{2}}\left(i k_{f}-\frac{1}{r}\right) \\
& J\left(\bar{\xi}_{m}, \bar{\eta}_{k}\right) \bar{J}\left(\bar{\xi}_{m}, \bar{\eta}_{k}\right) W_{m} W_{k}
\end{aligned}
$$

In which "W" are the Gaussian weights at integration points and $n p x, n p y$ are orders of integration in $x$ and $y$ directions ( $n p x=n p y=9$ for this chapter). In other words, to find the velocity at a target point the integrand should be computed at $n p x \times n p y$ points to evaluate the integral. The local coordinates of the integration points and their corresponding weights are available in the literature on finite element and numerical methods (see [75]). Similarly the pressure at target point due to an element source is derived in equations (3.18) and (3.19): 


$$
\begin{gathered}
\mathbf{P}_{e i}\left(\mathbf{r}_{\mathbf{t}}\right)=\sum_{n=1}^{2} \int_{-1}^{1} \int_{-1}^{1} N_{i}(\xi, \eta) \frac{\exp \left(i k_{f} r\right)}{r} J(\xi, \eta) \bar{J}(\bar{\xi}, \bar{\eta}) d \bar{\xi} d \bar{\eta} \\
\mathbf{P}_{e}\left(\mathbf{r}_{\mathbf{t}}\right)=\sum_{i=1}^{n} A_{S i} \mathbf{P}_{e i}\left(\mathbf{r}_{\mathbf{t}}\right)
\end{gathered}
$$

\subsubsection{ESM for modeling ultrasonic transducers}

A MATLAB code is developed to model a rectangular transducer with the ESM method. The transducer is discretized into $n \times m$ number of elements; therefore, it has $(n+1) \times(m+1)$ nodes with unknown source strengths. The boundary condition to be satisfied is the uniform normal velocity at the nodal points. Using equations (3.9) and (3.12), $(n+1) \times(m+1)$ unknowns are determined from $(n+1) \times(m+1)$ equations from the boundary condition at element nodes. After knowing the nodal values the pressure field is obtained using equations (3.18) and (3.19).

Since there is no closed form analytical solution for the axial pressure field for a rectangular transducer vibrating with uniform velocity, the Rayleigh-Sommerfeld integral (RSI) is integrated numerically to compare the integral value with the ESM predicted results:

$$
p(\mathbf{x})=-\frac{i \omega \rho v_{0}}{2 \pi} \int_{S} \frac{\exp \left(i k_{f} r\right)}{r} d S
$$

Comparing Eqs. (3.20) and (3.6) one can conclude that ideally the nodal source strength should have the following value:

$$
A_{s}=-\frac{i \omega \rho v_{0}}{2 \pi}
$$




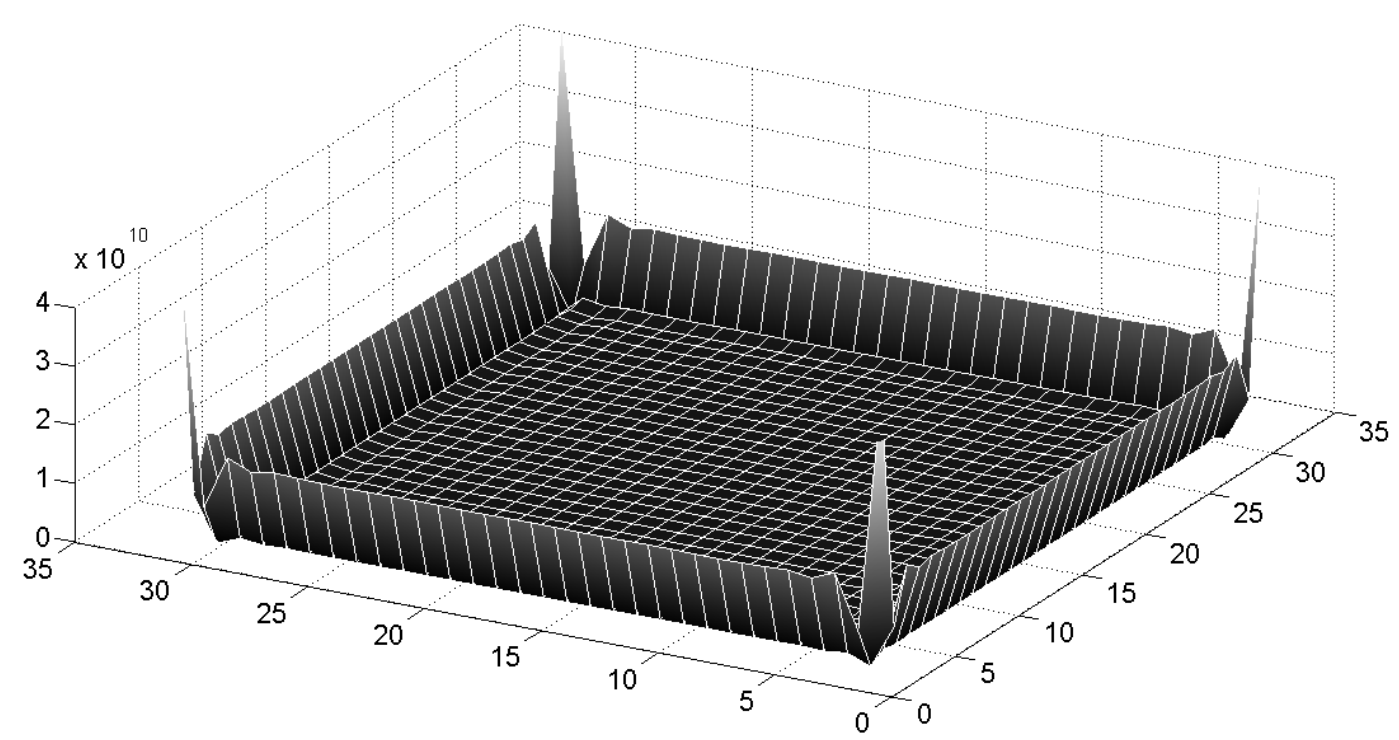

Figure 3.8 Nodal source strength variation: $1.55 \mathrm{~mm} \times 1.55 \mathrm{~mm}$ transducer, uniform velocity boundary condition

A rectangular transducer vibrating at $5 \mathrm{MHz}$ is modeled. By solving the equations from the uniform normal velocity boundary condition the nodal source strength are computed. Figure 3.8 shows the absolute values of the nodal source strength for a $1.55 \mathrm{~mm} \times 1.55$ mm transducer divided into $30 \times 30$ elements. The interior $A_{s}$ values are close to the expected value given in equation (3.21) but at corner and edge nodes there is a big jump that results in a significant error in the computed axial pressure field as shown in Figure 3.9. When the number of elements is increased from $30 \times 30$ to $34 \times 34$ the jump increased and the computed error did not decrease.

To understand the reason for this mismatch the velocity in the $\mathrm{z}$ direction is obtained from the pressure field given in equation (3.20) (see [26, 27, 41, 64]): 


$$
v_{z}(\mathbf{x})=-\frac{v_{0} z}{2 \pi} \int_{S} \frac{\exp \left(i k_{f} r\right)}{r^{3}}\left(i k_{f} r-1\right) d S
$$

The absolute value of the normal velocity on a plane very close to the transducer surface and parallel to it is computed using the numerical integration of equation $(3.22)\left(v_{0}=1\right)$, and plotted in Figure 3.10. The velocity is uniform except at the corner points and edges. It is the Gibbs effect at the edges because with a finite number of numerical integration elements, we are expanding the field in terms of a finite number of complex exponentials. Such a sum will converge to the mean value of the velocity discontinuity at a straight edge. In order to overcome the error in the pressure field computation shown in Figure 3.9, instead of setting unit normal velocity on the transducer surface the boundary condition shown in Figure 3.10 is assigned.

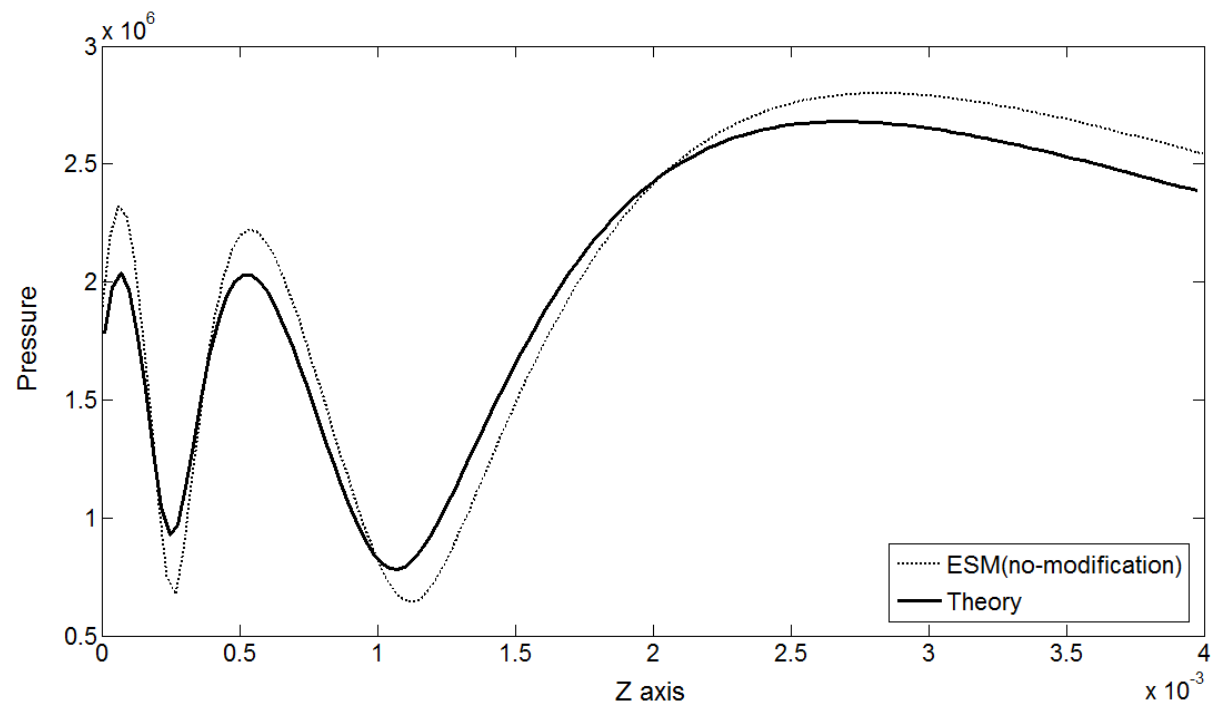

Figure 3.9 Axial pressure variation: $1.55 \mathrm{~mm} \times 1.55 \mathrm{~mm}$ transducer vibrating at $5 \mathrm{MHz}$ : Theory - continuous line; ESM (30x30 elements) for uniform velocity boundary condition - dotted line 


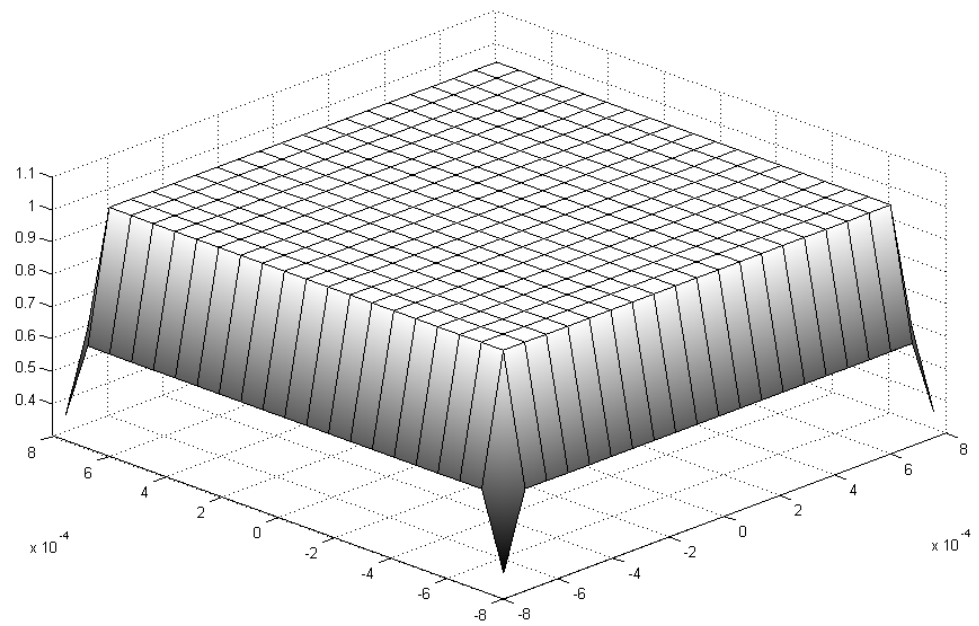

Figure 3.10 Modified boundary condition; $1.55 \mathrm{~mm}$ x $1.55 \mathrm{~mm}$ transducer vibrating at $5 \mathrm{MHz}$

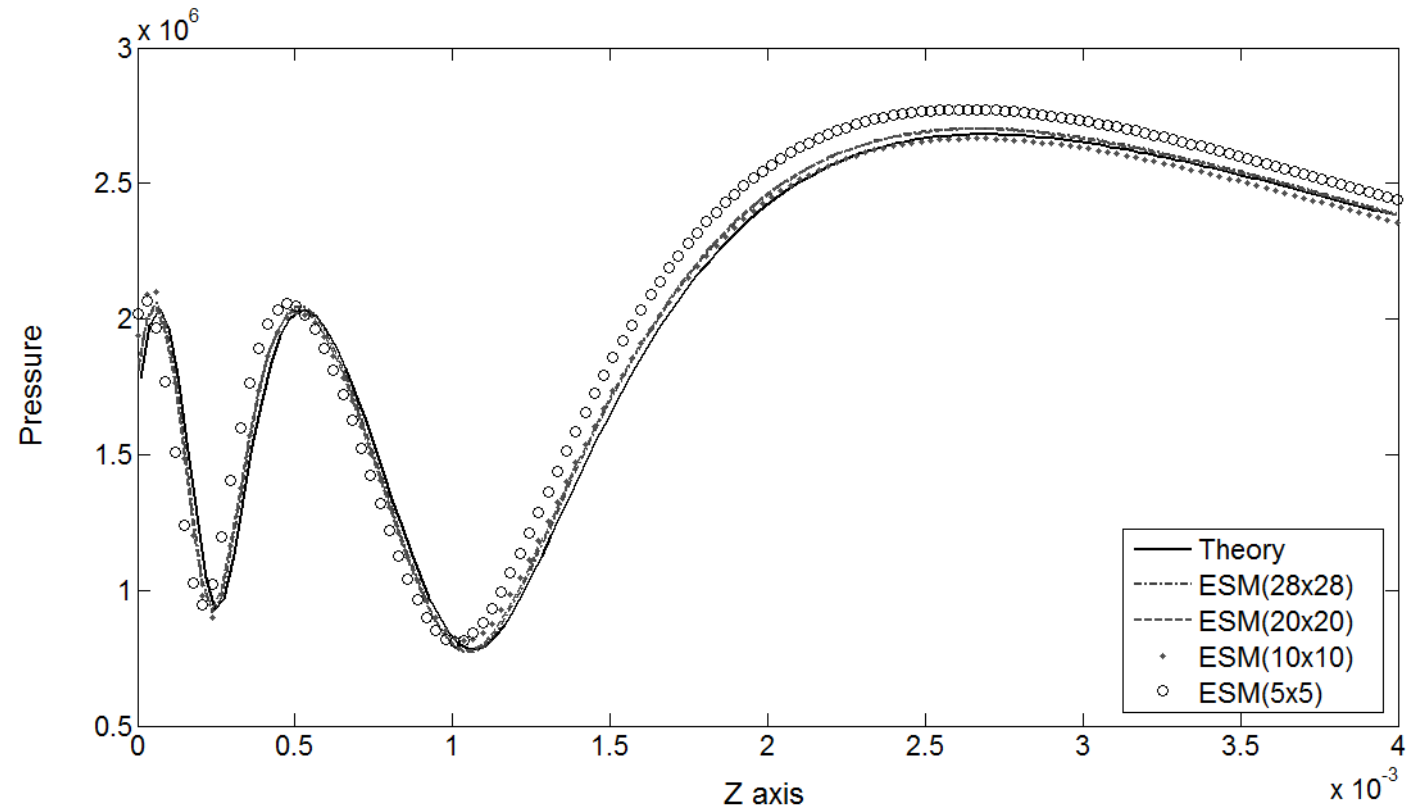

Figure 3.11 Axial pressure variation: $1.55 \mathrm{~mm} \times 1.55 \mathrm{~mm}$ transducer vibrating at $5 \mathrm{MHz}$ : continuous line Theory; other curves - ESM predictions with modified boundary conditions when divided into 28x28, 20x20, $10 \times 10$ and $5 \times 5$ elements 
With this boundary condition a better result is expected since the same boundary condition now dictates the numerically computed theory in equation (3.20) and the ESM. Figure 3.11 shows a comparison between the analytical pressure field and the ESM predictions for $1.55 \mathrm{~mm} \times 1.55 \mathrm{~mm}$ transducer divided into $28 \times 28,20 \times 20,10 \times 10$ and $5 \times 5$ elements and considering the modified boundary condition of Figure 3.10. The ESM prediction converges to the theory as the number of elements is increased. With more than $10 \times 10$ elements the ESM prediction agrees very well with the theory. Figure 3.12 shows scaled DPSM results for $34 \times 34,28 \times 28,20 \times 20$ and $10 \times 10$ point sources along with the theoretical result. It should be noted that 10x10 DPSM results did not converge but finer mesh 20x20 and higher converged very well in the far field but not so well near the two dips in the near field while the ESM results even with 10x10 mesh converged very well in both near and far fields as shown in Figure 3.11.

ESM (20x20) and DPSM (34×34) results are shown on top of each other with the theoretical curve in Figure 3.13. This figure clearly shows that the DPSM generated curve with $34 \times 34$ point sources does not match with the theoretical curve near the two dips of the near field (near $\mathrm{z}=0.25 \mathrm{~mm}$ and $1 \mathrm{~mm}$ ) as good as the ESM results do with only 20x20 elements. Therefore, we can conclude that ESM requires significantly fewer DOFs in comparison to DPSM to produce accurate results 


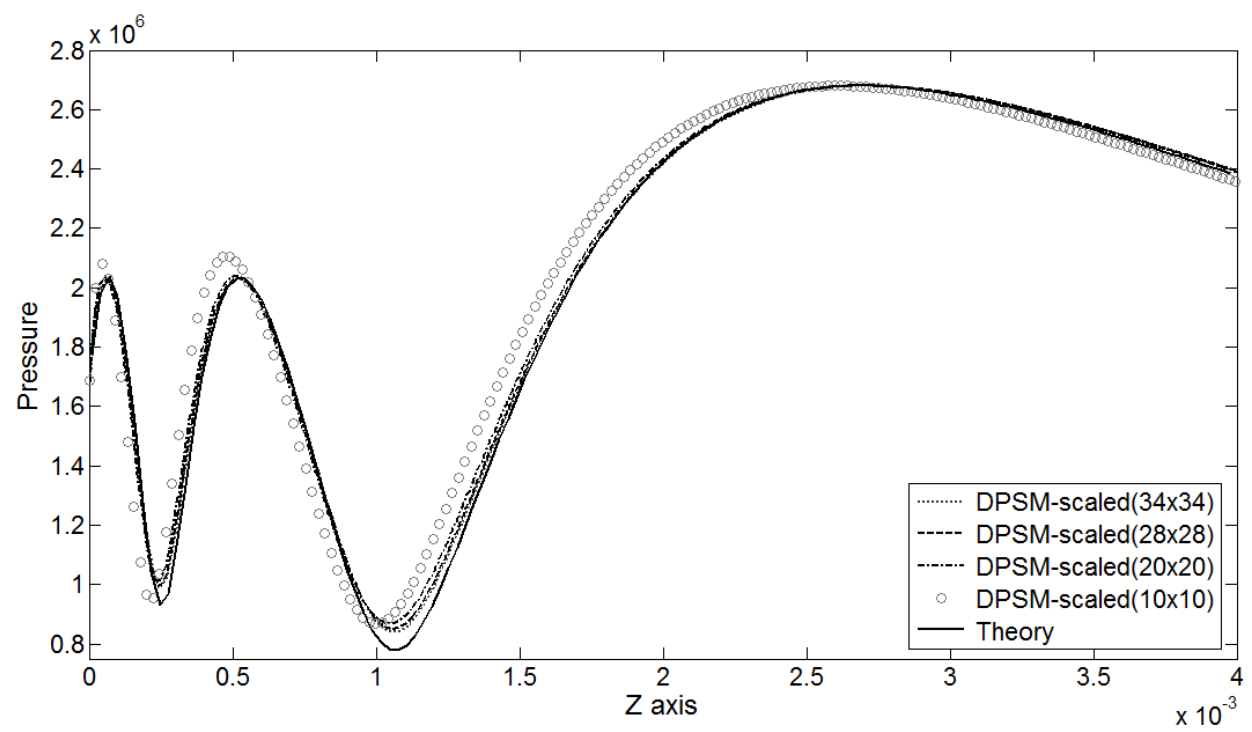

Figure 3.12 Axial pressure variation: $1.55 \mathrm{~mm}$ x $1.55 \mathrm{~mm}$ transducer vibrating at $5 \mathrm{MHz}$ : continuous line Theory; other curves show DPSM predictions, generated by 34x34, 28x28, 20x20 and 10x10 point sources

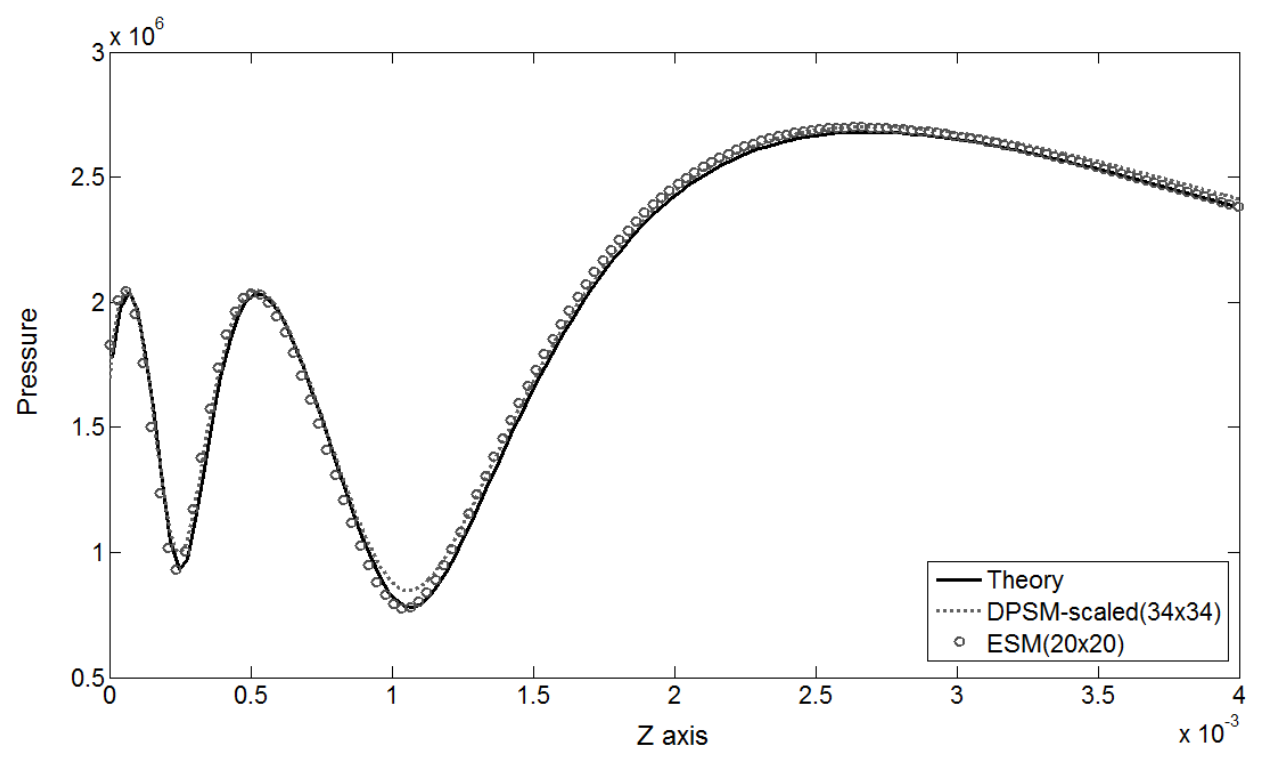

Figure 3.13 Axial Pressure variation: $1.55 \mathrm{~mm} \times 1.55 \mathrm{~mm}$ transducer vibrating at $5 \mathrm{MHz}$ - computed results by ESM (20x20 element sources) and DPSM (34x34 point sources) are compared with the theoretical curve 


\subsection{Proposed Element Source Method (ESM) in Electromagnetics}

In section 1.7.3 the electromagnetic Gaussian beam propagation has been modeled using DPSM. Figure 3.14 compares the analytical solution for the Gaussian beam in equation (1.88) at $0.5 \mathrm{THz}$ frequency with DPSM solution. It is shown that by increasing the source radius the DPSM solution does not converges to analytical one but to the scaled form of the analytical curve.

In this section Element Source Method (ESM) is proposed to bypass the scaling needs. ESM methods are illustrated by computing the electric field in front of circular Gaussian beam emitter and comparing the computed results with the available analytical solutions.

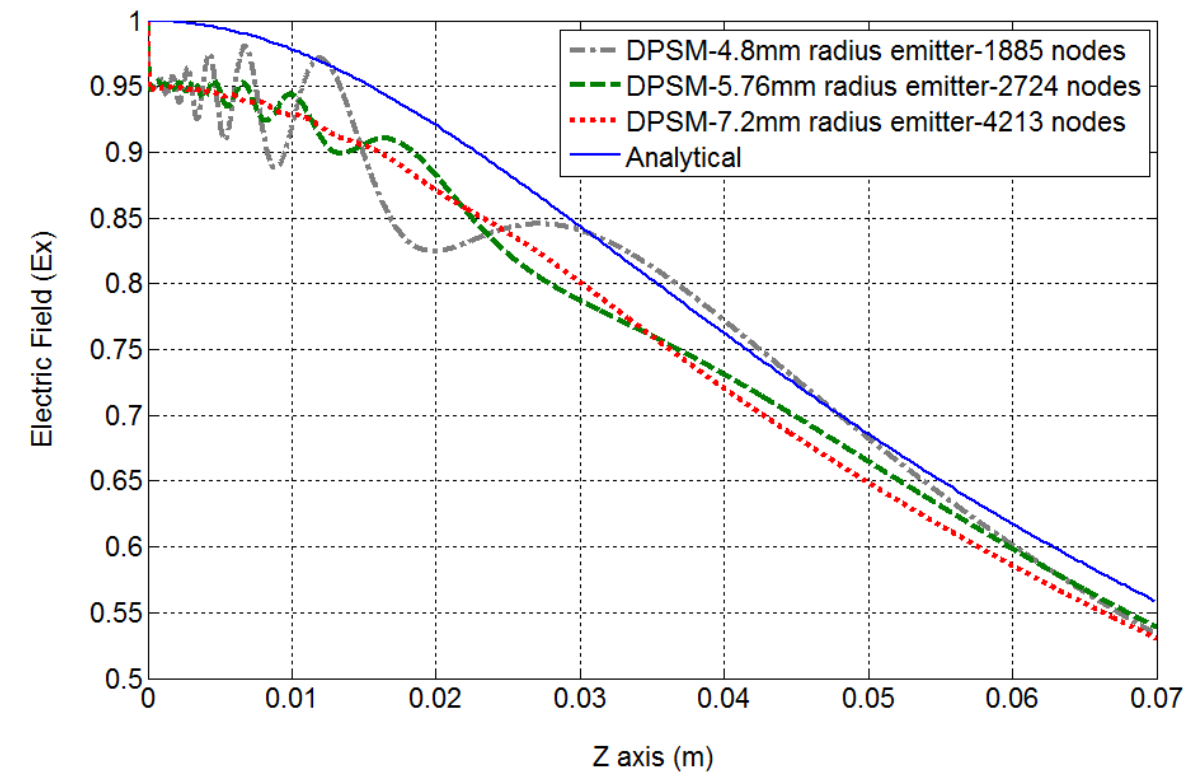

Figure 3.14 Gaussian Beam axial Electric Field at 0.5 THz frequency (Comparison between DPSM and Analytical solution). 
As shown in Figure $3.15 \mathrm{a}$ if $\mathbf{J}_{\mathrm{s} 1}, \mathbf{J}_{\mathrm{s} 2}$ and $\mathbf{J}_{\mathrm{s} 3}$ are $3 \times 1$ source strength vectors at 3 nodes of a linear triangular element then the source strength within the element can be expressed

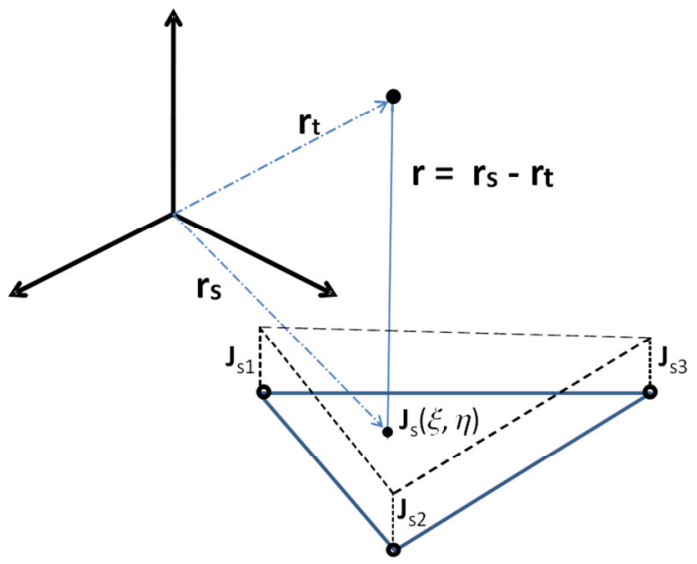

(a)
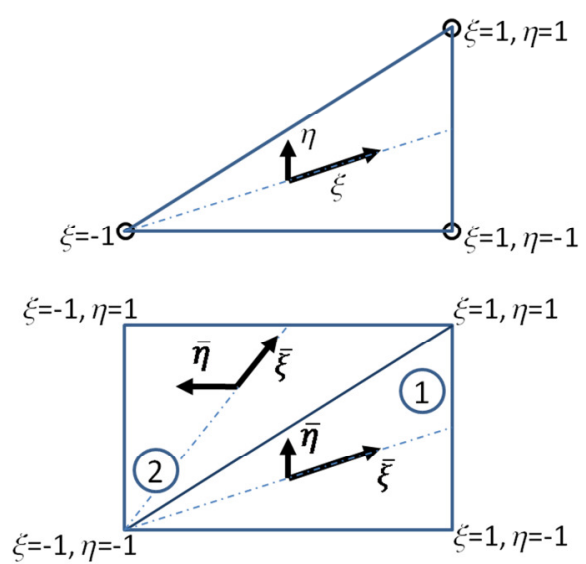

(b)

Figure 3.15 (a) Schematic depiction of a source element in global coordinate (b) Main element and the subelements in local coordinate

in terms of the local coordinates and linear shape functions or interpolation functions as used in finite element and boundary element methods:

$$
\mathbf{J}_{s}(\xi, \eta)=\sum_{i=1}^{n} \mathbf{J}_{s i} N_{i}(\xi, \eta)
$$

$n=$ number of element nodes ( $n=3$ for a linear triangular element)

$$
\begin{gathered}
N_{1}=\frac{1}{4}(1+\xi)(1+\eta) \\
N_{2}=\frac{1}{4}(1+\xi)(1-\eta) \\
N_{3}=\frac{1}{2}(1-\xi)
\end{gathered}
$$


Quadratic shape functions should be used for quadric (6 node) elements. The unknown parameters in ESM formulation are the source strengths at the element nodes. For linear and quadric elements 3 and 6 nodes of the element represent 3 and 6 point sources, respectively with unknown strengths. Since the source strength is defined continuously using the continuous interpolation functions, in the ESM formulation the compatibility and boundary conditions can be satisfied at non-nodal points as well.

The position vector of a point in the element with $(\xi, \eta)$ coordinate is given by:

$$
\mathbf{r}_{s}(\xi, \eta)=\sum_{i=1}^{3} \mathbf{r}_{s i} N_{i}(\xi, \eta)
$$

The electric field at a target point is computed by integrating the corresponding Green's functions in equation (1.118) over the element:

$$
\mathbf{E}_{e}\left(\mathbf{r}_{\mathbf{t}}\right)=\int[\mathbf{E M}] \mathbf{J}_{s} d s
$$

In order to be able to carry out the integration over the element the local coordinate and Jacobian transformation matrix are implemented:

$$
\mathbf{E}_{e}\left(\mathbf{r}_{\mathbf{t}}\right)=\int_{-1-1}^{1} \int_{-1}^{1}[\mathbf{E M}] \mathbf{J}_{s}(\xi, \eta) t(\xi, \eta) d \xi d \eta
$$

In which:

$$
\mathbf{V}=\left[\begin{array}{l}
\frac{\partial y}{\partial \xi} \frac{\partial z}{\partial \eta}-\frac{\partial y}{\partial \eta} \frac{\partial z}{\partial \xi} \\
\frac{\partial z}{\partial \xi} \frac{\partial x}{\partial \eta}-\frac{\partial z}{\partial \eta} \frac{\partial x}{\partial \xi} \\
\frac{\partial x}{\partial \xi} \frac{\partial y}{\partial \eta}-\frac{\partial x}{\partial \eta} \frac{\partial y}{\partial \xi}
\end{array}\right] \quad, \quad t(\xi, \eta)=|\mathbf{V}|
$$


The integration in equation (3.27) has no closed form solution so Gaussian quadrature numerical integration technique is used.

The integrand in equation (3.27) is singular (when $r=0$ ). To avoid this singularity the source points are shifted by an amount " $r_{s}$ " (see [26]) from the boundary as shown in Figure 1.22. After the source shifting, in order to get more accurate numerical integration results the element is divided into two sub-elements (see Figure 3.15b) and a local coordinate system is introduced. The local coordinate system is chosen in such a way that the Jacobian of the transformation approaches zero at element nodes (see [74]). This method gives a more uniform integrand within the element and finally more accurate integration value. This approach is widely used in boundary element method to solve the singularity problem during integration (see [pages 157-160 of Ref. [74]]); finally, integrating over the two sub elements we get:

$$
\mathbf{E}_{e i}\left(\mathbf{r}_{\mathbf{t}}\right)=\sum_{n=1}^{2} \int_{-1-1}^{1} \int_{-1}^{1} N_{i}(\xi, \eta)[\mathbf{E M}] t(\xi, \eta) \bar{t}(\bar{\xi}, \bar{\eta}) d \bar{\xi} d \bar{\eta}
$$

In which the shape function converting the sub-element local coordinate $(\bar{\xi}, \bar{\eta})$ to the main element local coordinate $(\xi, \eta)$ reads like:

$$
\begin{gathered}
\xi=\sum_{i=1}^{3} \xi_{i} \bar{N}_{i}(\bar{\xi}, \bar{\eta}) \\
\eta=\sum_{i=1}^{3} \eta_{i} \bar{N}_{i}(\bar{\xi}, \bar{\eta}) \\
\bar{N}_{1}=\frac{1}{4}(1+\bar{\xi})(1-\bar{\eta}) \quad \bar{N}_{2}=\frac{1}{4}(1+\bar{\xi})(1+\bar{\eta}) \\
\bar{N}_{3}=\frac{1}{2}(1-\bar{\xi})
\end{gathered}
$$


Also the Jacobian transformation from Element to Sub-element coordinate is:

$$
\bar{t}=\left|\begin{array}{ll}
\frac{\partial \xi}{\partial \bar{\xi}} & \frac{\partial \eta}{\partial \bar{\xi}} \\
\frac{\partial \xi}{\partial \bar{\eta}} & \frac{\partial \eta}{\partial \bar{\eta}}
\end{array}\right|
$$

Finally the Gaussian quadrature integration form of equation (3.29) gives:

$$
\begin{gathered}
\mathbf{E}_{e i}\left(\mathbf{r}_{\mathbf{t}}\right)=\sum_{n=1}^{2} \sum_{m=1}^{n p x} \sum_{k=1}^{n p y} N_{i}\left(\bar{\xi}_{m}, \bar{\eta}_{k}\right)[\mathbf{E M}] t\left(\xi_{m}, \eta_{k}\right) \bar{t}\left(\bar{\xi}_{m}, \eta_{k}\right) W_{m} W_{k} \\
\mathbf{E}_{e}\left(\mathbf{r}_{\mathbf{t}}\right)=\sum_{i=1}^{3} \mathbf{J}_{s i} \mathbf{E}_{e i}\left(\mathbf{r}_{\mathbf{t}}\right)
\end{gathered}
$$

In which "W" are the Gaussian weights at integration points and npx, npy are orders of integration in $x$ and $y$ directions ( $n p x=n p y=9$ for this case study). In other words, to find the velocity at a target point the integrand should be computed at $n p x \times n p y$ points to evaluate the integral. The local coordinates of the integration points and their corresponding weights are available in the literature on finite element and numerical methods (see [75]).

\subsubsection{ESM Gaussian beam modeling results}

Modeling a Gaussian beam has been chosen as a case study to verify the proposed ESM for electromagnetic problems. As shown in Figure 3.16 the Gaussian beam source is discretized into triangular elements; therefore, it has 'n' nodes with unknown source strengths. Similar to DPSM, the boundary condition to be satisfied is the electric field 


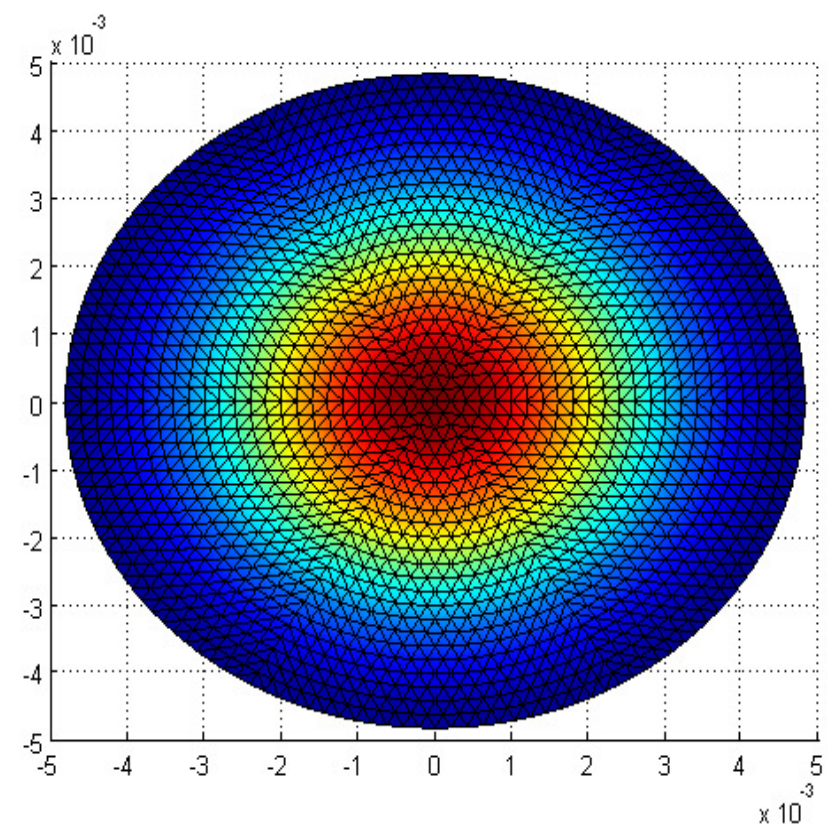

Figure 3.16 Meshing the $4.8 \mathrm{~mm}$ radius transducer by triangular elements and Gaussian Electric field boundary condition on surface

using equation (1.120). Using equation (3.34) and (1.120), 3n unknowns are determined from $3 \mathrm{n}$ equations from the boundary condition at element nodes. After knowing the nodal values the electric field is obtained using equation (3.34).

Figure 3.17 compares the analytical solution for the Gaussian beam in equation (1.88) at $0.5 \mathrm{THz}$ frequency with ESM solution. It is shown that by increasing the source radius and keeping the element size (which results in increasing number of elements) the ESM solution converges to analytical without scaling need. 


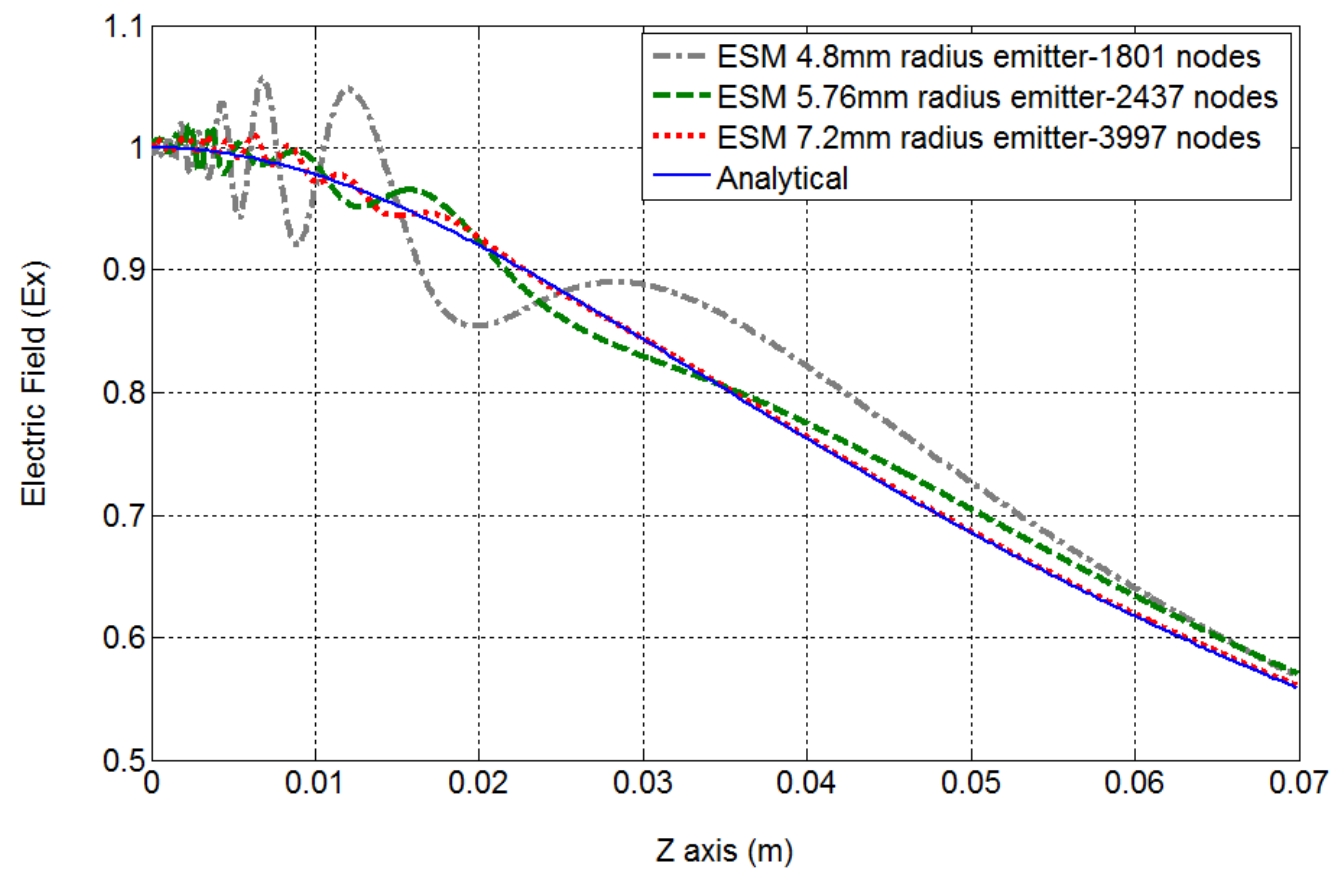

Figure 3.17 Gaussian Beam axial Electric Field at 0.5 THz frequency (Comparison between ESM and Analytical solution).

\subsection{Conclusions}

Proposed Gaussian DPSM (G-DPSM) and element source method (ESM) offer significant advantages over the conventional numerical methods like FEM and BEM. Advantages of DPSM over FEM for solving ultrasonic wave propagation problems have been established earlier in other publications on DPSM [28]. Advantages of G-DPSM and ESM over DPSM are emphasized in this chapter. Advantage of ESM over G-DPSM is also mentioned here for ultrasonic problems. It should be noted that there is a major difference between ESM and BEM. In ESM every element is considered as a finite source and superimposing the effects of all elements the total field is constructed while in BEM boundary integral equation formulation is required. The singularity of Green's 
functions is avoided by shifting the point sources by a small distance from the transducer face. ESM prediction matches very well with the analytical solutions both in the near field and in the far field. ESM needs a significantly fewer number of elements in comparison to DPSM and G-DPSM. As a result, ESM requires less computational time and computer memory. These two proposed modifications G-DPSM and ESM have been tested on circular and rectangular transducer geometries in sections 3.3 and 3.4.

In section 3.5 ESM technique used to model Gaussian $\mathrm{THz}$ beam produced results that matched very well with the analytical solutions both in the near field and the far field. Unlike DPSM, ESM generated field does not require scaling.

\subsection{Future Works}

In the future these methods can be generalized for modeling more complex ultrasonic and electromagnetic problem geometries containing interfaces and scatterers. For ultrasonic problems ESM has been developed to solve fluid medium problems. Later it can be extended to solid medium or problem geometries having both fluid and solid media. 


\section{CHAPTER 4}

\section{DPSM FOR SOLVING ACOUSTIC WAVE SCATTERING PROBLEMS}

\subsection{Introduction:}

Detecting cavities and inclusions in fluid and solid media is a fundamental problem in nondestructive testing and evaluation, and exploration geophysics. A large number of papers have been published on elastic wave scattering by inclusions in a material [76], [77]. In particular, scattering of elastic waves by spherical inhomogeneities - voids and inclusions - have been investigated extensively because of its relatively simple geometry and yet having potential applications in different fields [76]. Tittman et al. [78] studied the scattering of elastic waves by spherical cavities for detection, identification, and characterization of defects in solids; they studied the longitudinal wave scattering by a spherical cavity in a solid and compared the experimental observations with their calculations for a single embedded cavity. Then they extended their work to the hard inclusion case [79]. Detection of a bubble (or a cavity) has been investigated more extensively when the bubble is located in a liquid. Although the single cavity geometry is good for theoretical analysis, ultrasonic signals reflected from individual bubbles often cannot be detected experimentally because of the lack of high spatial resolution and signal-to-noise ratio (SNR) needed for such measurements. To increase the spatial resolution, spherical focused lenses have been used by Ihara et al. [80-82]. They used 10 $\mathrm{MHz}$ focused ultrasound to detect 20 to $80 \mu \mathrm{m}$ diameter particles in molten zinc at 
$650^{\circ} \mathrm{C}$. The interaction between a spherical cavity and a focused ultrasonic beam has been investigated analytically by Lobkis et al. [48, 83] and Zinin et al. [35, 84]. These analytical investigations have several restrictions that make the analytical methods applicable only to high frequency focused signals interacting with a small spherical cavity having a small eccentricity or offset from the focal point. For a cavity in a solid medium the analytical solution is even more restrictive such as signal duration should be sufficiently short such that the longitudinal and transverse waves scattered by the cavity are separated in time and can be analyzed separately [35, 48, 83, 84]. Without these simplifying assumptions there is no analytical solution for this problem. The interaction between a cavity or void in a liquid and a converging ultrasonic beam generated by a point focused acoustic lens has been investigated by Placko et al. [49], they studied the effect of a bubble present in a liquid using different analytical and numerical techniques and compared the results. This work was then extended by Hajzargarbashi et al. [85] to study the Scattering of focused ultrasonic beams by two spherical cavities in close proximity in a fluid. In these works a newly developed mesh-free technique called Distributed Point Source Method or DPSM was used to model the acoustic field. The DPSM technique for solving ultrasonic, electrostatic and electromagnetic problems was developed by Placko and Kundu [26, 62]. Using DPSM, the ultrasonic field has been modeled in fluid media [28, 29, 41] and solid structures [26, 43, 44]. Kundu et al. [86] successfully modeled the ultrasonic field generated in front of an acoustic lens in a perfect fluid medium in absence of any anomaly. Placko et al [49]. Hajzargarbashi et al. 
[85] and Rahani and Kundu [87] have investigated the interaction between focused ultrasonic beam with single and multiple cavities in a fluid medium.

In section 4.2 of this chapter the advantages of DPSM over Finite element method (FEM) are highlighted. The DPSM technique is then extended to model the ultrasonic field generated by a concave lens placed in a fluid medium adjacent to the solid halfspace containing cavities. To obtain accurate information about the size and location of the cavities in a solid using an acoustic microscope, it is necessary to correctly model the interaction between the cavities, the fluid-solid interface and the ultrasonic beam generated by the acoustic microscope lens. The effect of the location of the cavity relative to the focal point of the lens and the solid-liquid interface is studied. Then the investigation is extended to the case of two cavities in close proximity. The interaction between the cavities, the lens and the interface is also investigated.

\subsection{Advantages of DPSM over FEM in Wave Propagation Problems}

Semi-analytical results obtained by the DPSM technique and the numerical results obtained from the finite element analysis (using COMSOL MultiPhysics software [88]) are compared with the theoretical results. In Figure 4.1 the pressure along the central axis of a circular transducer is plotted from all three analyses - analytical, semi-analytical and numerical. Results generated by the DPSM analysis with 600 point sources is shown by the continuous line while the dotted line shows the theoretical curve and the finite element results are shown by the dashed line. Since this problem is axi-symmetric the finite element result could be obtained relatively easily from the two-dimensional axi- 
symmetric analysis with 174,927 triangular elements. In Figure 4.1 one can see that the error in DPSM and FEM results are of the same order that can be reduced further by

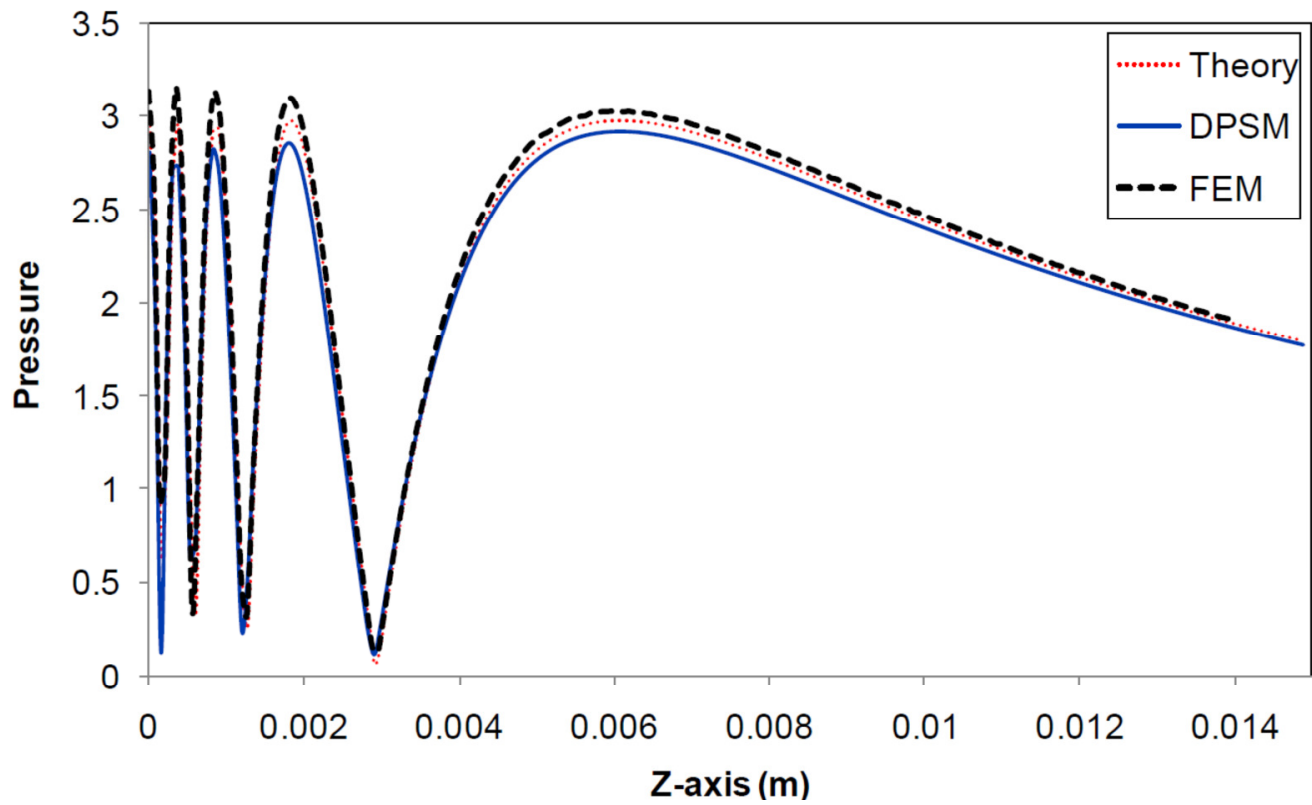

Figure 4.1 Pressure field variation along the central axis of a flat circular transducer : Comparison between the theoretical curve (dotted line), finite element solution (dashed line) and DPSM solution (continuous line). The scale for the vertical axis is Pascal for transducer surface velocity amplitude $1 \mu \mathrm{m} / \mathrm{s}$ (see [28]).

increasing the number of point sources in the DPSM analysis or the number of elements in the FEM analysis.

Figure 4.2 shows the pressure variation along the central axis of a circular concave lens with $4 \mathrm{~mm}$ radius of curvature and $40^{\circ}$ lens angle. Because of the axisymmetric nature of the problem the two-dimensional axi-symmetric finite element model could be used in this case also. The DPSM curve was generated with 600 point sources while 75,893 triangular finite elements were used to generate the numerical curve. Note that both DPSM and FEM results match equally well with the theoretical curve. 


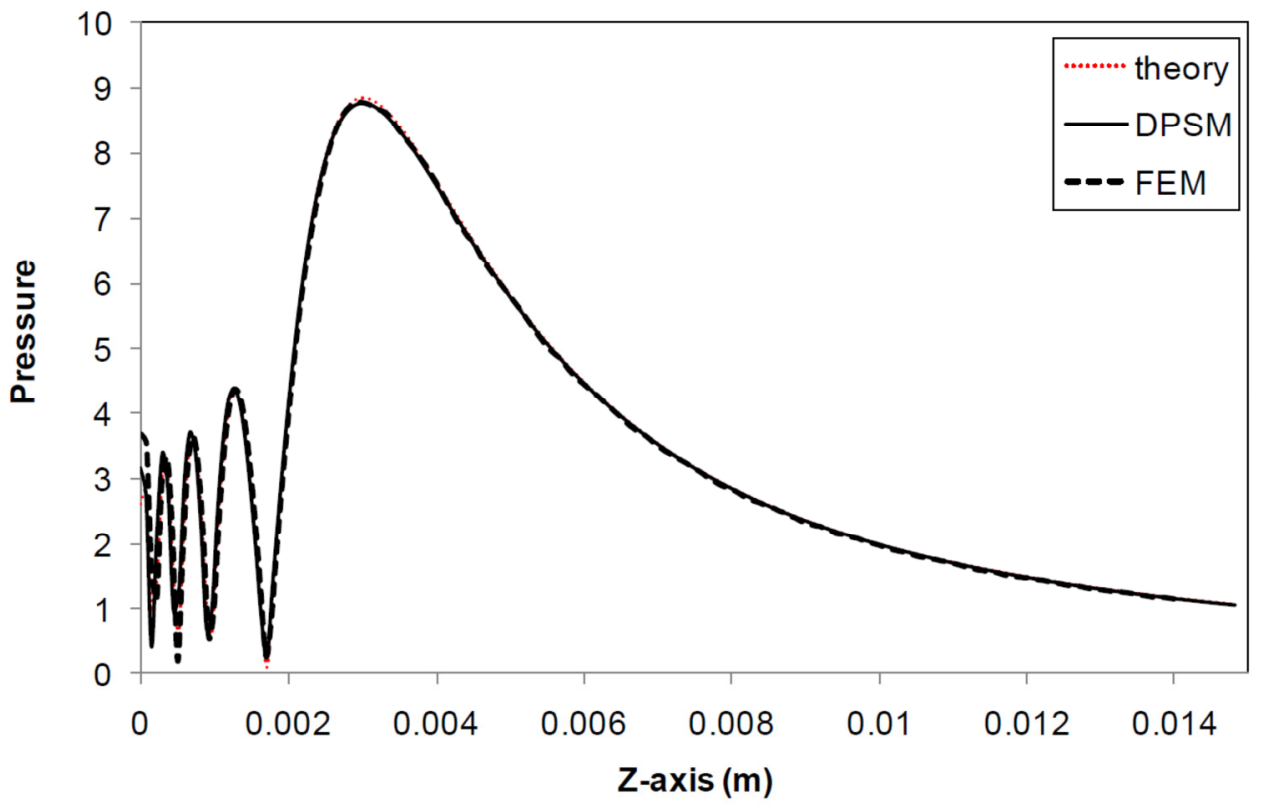

Figure 4.2 Pressure field variation along the central axis of a concave point focused circular transducer : Comparison between the theoretical curve (dotted line), finite element solution (dashed line) and DPSM solution (continuous line). The radius of curvature of the transducer is $4 \mathrm{~mm}$ and it forms $\mathbf{4 0}^{\circ}$ angle at the center of curvature. All three curves coincide. The scale for the vertical axis is Pascal for transducer surface velocity amplitude $1 \mu \mathrm{m} / \mathrm{s}$ (see [28]).

Pressure fields in front of square transducers are then computed. Figure 4.3 shows the field for a $0.5 \mathrm{~mm} \times 0.5 \mathrm{~mm}$ square transducer. No closed form analytical solution exists for this transducer geometry. Since DPSM technique can be applied to any transducer geometry the DPSM solution with uniform source strength is treated as the ideal solution or the RSI solution. This result is shown by the dotted line in Figure 4.3. The continuous line shows the DPSM results when the source strengths are obtained by matching the prescribed velocity condition on the transducer surface. This result is obtained with 1225 point sources modeling the transducer face. The dashed line is obtained from the finite 
element analysis. Since this problem geometry is not axi-symmetric the two-dimensional finite element model could not be used here.

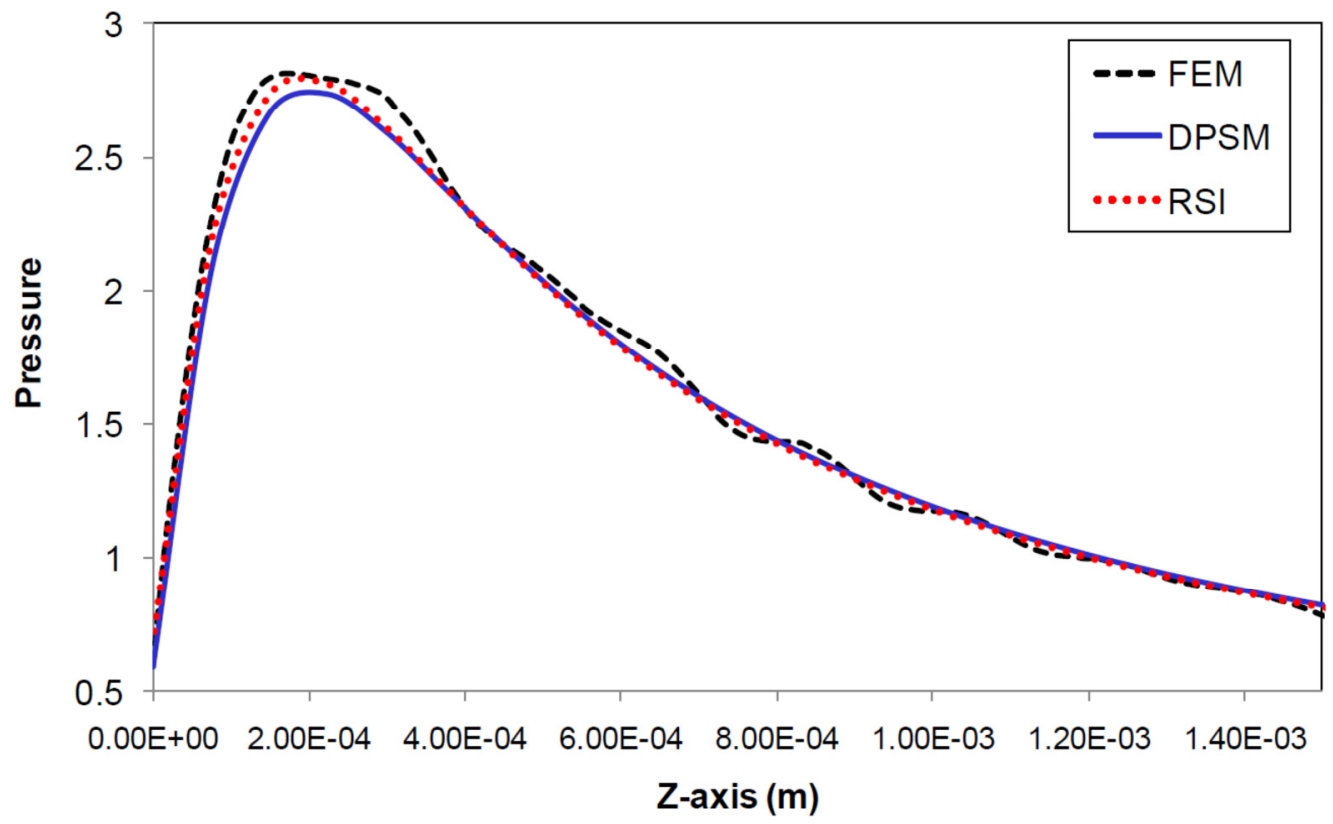

Figure 4.3 Pressure field variation along the central axis of a flat square transducer: Comparison between the RSI solution (dotted line), finite element solution (dashed line) and DPSM solution (continuous line). The transducer is $0.5 \mathrm{~mm} \times 0.5 \mathrm{~mm}$ and the signal frequency is $5 \mathrm{MHz}$. Note that the DPSM matches better with the

RSI solution. The Scale for the vertical axis is Pascals for transducer surface velocity amplitude $1 \mu \mathrm{m} / \mathrm{s}$ (see [28]).

Three-dimensional finite element mesh with 146,098 3D tetrahedral elements was used to generate the numerical results. Note that in this case DPSM gives better results than FEM.

As the square transducer size is increased from $0.5 \mathrm{~mm}$ side length to $1.55 \mathrm{~mm}$ side length the finite element method encountered some difficulty but the DPSM solution did not. Results for the $1.55 \mathrm{~mm} \times 1.55 \mathrm{~mm}$ square transducer are shown in Figure 4.4a. DPSM results, shown as a continuous line, matched very well with the RSI solution, the dotted line. 
However, the three-dimensional finite element mesh with $752,6813 \mathrm{D}$ tetrahedral elements had difficulty in converging as shown by the dashed line.

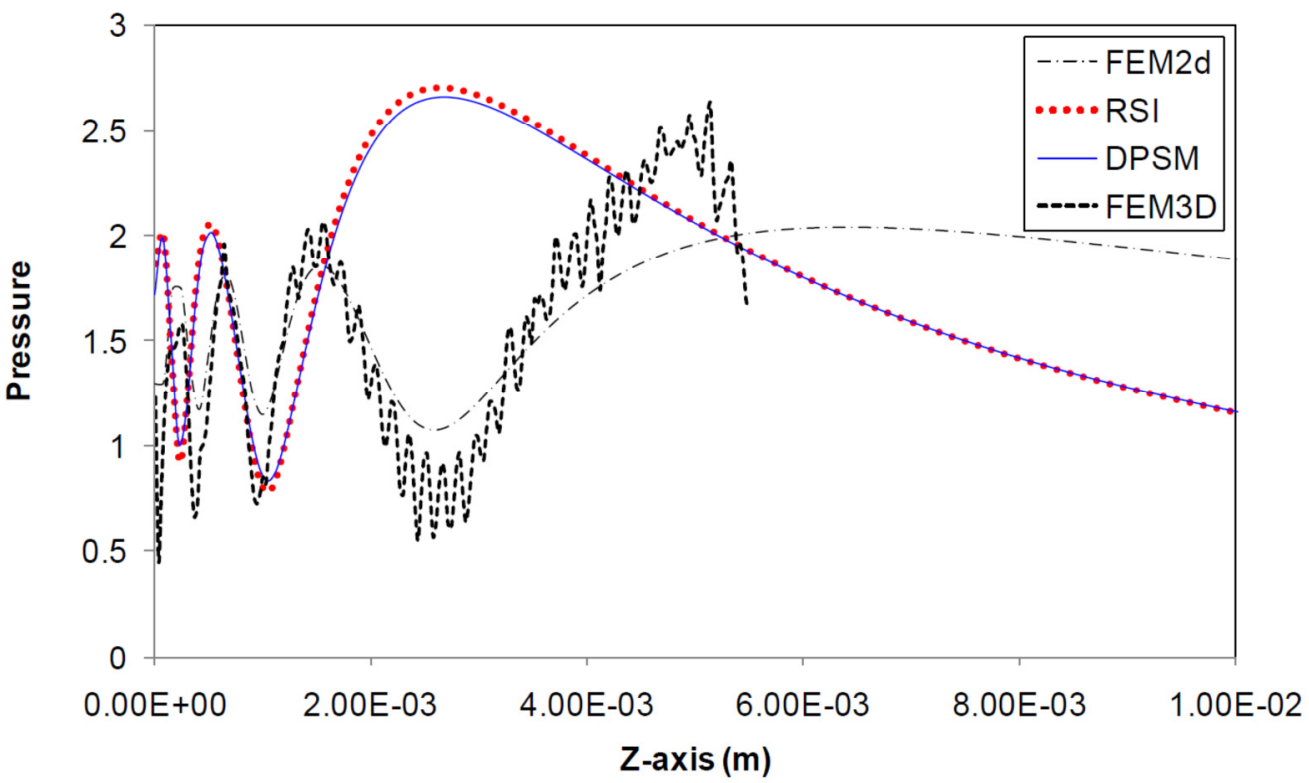

(a)

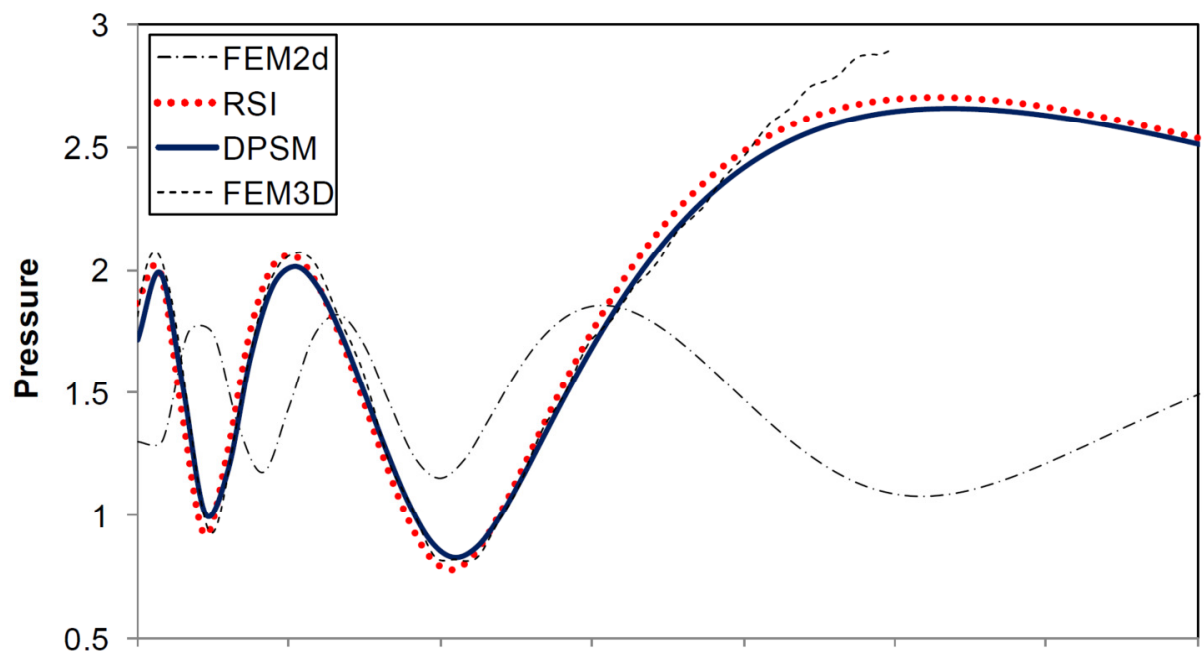

0.00E+00 5.00E-04 1.00E-03 1.50E-03 2.00E-03 2.50E-03 3.00E-03 3.50E-03

Z-axis (m)

(b)

Figure 4.4 Pressure field variation along the central axis of a flat square transducer: comparison between the RSI solution (dotted line), finite element solution (dashed line), and DPSM solution (continuous line). The transducer is $1.55 \times 1.55 \mathrm{~mm}$ and the signal frequency is $5 \mathrm{M} \mathrm{Hz}$. A fourth curve (dashed-dotted line) is generated by 2-D finite element model for a $1.5-\mathrm{mm}$-wide transducer. Neither the 2-D nor 3-D finite element model produces the RSI solution. The scale for the vertical axis is pascals for transducer surface velocity amplitude $1 \mu \mathrm{m} / \mathrm{s}$. (b) Pressure field variation along the central axis of a flat square $(1.55 \times 1.55 \mathrm{~mm})$ 
transducer: as in (a), except that the computation is carried out only up to $3.5 \mathrm{~mm}$ in the axial direction instead of $10 \mathrm{~mm}$ to have much smaller finite elements. The 3-D finite element analysis now shows better matching with the RSI and DPSM solutions compared with that in (a). The scale for the vertical axis is pascals for transducer surface velocity amplitude $1 \mu \mathrm{m} / \mathrm{s}$ (see [28]).

It was also investigated how well a two-dimensional finite element analysis can model this transducer. A two-dimensional plane strain finite element model with 234,785 triangular elements generated the dashed-dotted line of Figure 4.4a. Clearly, the 2D approximation is not adequate for modeling this 3D problem. By discretizing a smaller volume in front of the transducer it was possible to generate the pressure field by the 3D finite element analysis as shown in Figure 4.4b. The finite element result was obtained by discretizing a fluid volume of dimension $3 \times 3 \times 3 \mathrm{~mm} 3$ into 739,590 tetrahedral finite elements. It took 35 hours of computational time in a CPU Intel Xeon 2x2.66 GHz dual Core, $\mathrm{RAM}=6 \mathrm{~GB}$ computer. In spite of this massive computational effort the finite element solution still starts to deviate from the true solution at a distance greater than $2 \mathrm{~mm}$ from the transducer face.

\subsection{DPSM Theoretical Formulation}

When the problem geometry does not satisfy the restrictions of the analytical solution, such as when the cavity has a high eccentricity (in other words, when the cavity center is far away from the lens focal point), has a non-spherical shape, or when the cavity radius is not significantly smaller than the focal distance then the problem must be solved by numerical or semi-analytical techniques. The common numerical techniques such as the Finite Element Method, the Boundary Element Method and the Spectral Finite Element Method [54-59, 89] become computationally very time consuming at high 
frequencies. DPSM technique is extended here to model cavities in a solid half-space adjacent to the fluid medium containing the acoustic source.

In DPSM a number of point sources are distributed behind the transducer face to model the transducer. An interface generates reflected and transmitted ultrasonic fields. The interface is modeled by two layers of point source - one layer generating the reflected field and the second layer generating the transmitted field. Two layers of interface source are distributed on two sides of the interface. Strengths of the point sources distributed near the transducer face and the interface are obtained by simultaneously satisfying all boundary and interface continuity conditions. For solving this problem involving fluid and solid media we need point source solutions for both solid and fluid media. Therefore, the first step necessary for this technique is to calculate the stress and displacement Green's functions in the solid, and pressure and displacement Green's functions in the fluid.

\subsubsection{Computation of displacement and stress Green's functions in the solid}

For isotropic homogeneous solids the expression for stresses can be written as

$$
\sigma_{i j}=2 \mu \varepsilon_{i j}+\lambda \delta_{i j} \varepsilon_{k k}
$$

where $\lambda$ and $\mu$ are the two Lame' constants and $\delta_{i j}$ is the Kronecker delta. Strains in terms of displacement are

$$
\varepsilon_{i j}=\frac{1}{2}\left(u_{i, j}+u_{j, i}\right)
$$


It is well-known that due to a point source $p_{j}$ acting in direction $x_{j}$ at location $\mathbf{y}$ in a solid the displacement at location $\mathbf{x}$ in direction $x_{i}$ is given by [26]

$$
u_{i}=G_{i j}(\mathbf{x} ; \mathbf{y}) p_{j} e^{-i \omega t}
$$

where $\mathbf{p}$ is the point force vector having three components $p_{1}, p_{2}$ and $p_{3}$ and $G_{i j}(\mathbf{x} ; \mathbf{y})$ is the displacement Green's function for the isotropic homogeneous solid. Considering $r=$ $|\mathbf{x}-\mathbf{y}|$ the displacement Green's function can be written as

$$
G_{i j}(\mathbf{x} ; \mathbf{y})=\frac{1}{4 \pi \rho w^{2}}\left[\begin{array}{l}
\frac{e^{i k_{p} r}}{r}\left(k_{p}^{2} R_{i} R_{j}+\left(3 R_{i} R_{j}-\delta_{i j}\right)\left(\frac{i k_{p}}{r}-\frac{1}{r^{2}}\right)\right)+ \\
\frac{e^{i k_{s} r}}{r}\left(k_{s}^{2}\left(\delta_{i j}-R_{i} R_{j}\right)-\left(3 R_{i} R_{j}-\delta_{i j}\right)\left(\frac{i k_{s}}{r}-\frac{1}{r}\right)\right.
\end{array}\right]
$$

Where $R_{i}=\frac{x_{i}-y_{i}}{r}$

The displacement vector is given by

$$
\mathbf{u}=\underline{\mathbf{G}}(\mathbf{x} ; \mathbf{y}) \mathbf{P}
$$

If the unit excitation force at $\mathbf{y}$ acts in the $j$-th direction, then the displacement at $\mathbf{x}$ in the $i$-th direction can be given by $G_{i j}(\mathbf{x} ; \mathbf{y})$.

When a group of point sources distributed over a finite dimension in a solid are excited, the response at any point in the solid can be computed by superimposing the contribution of every point source. Therefore, the total displacement at any point from $M$ number of point sources distributed near the solid boundary can be written as 


$$
u_{i}=\sum_{m=1}^{M}\left(G_{i 1}^{m} P_{1}^{m}+G_{i 2}^{m} P_{2}^{m}+G_{i 3}^{m} P_{3}^{m}\right)=\sum_{m=1}^{M} \underline{\mathbf{G}_{i}{ }^{m}} \mathbf{P}^{m}
$$

Substituting the expression for displacement in the strain-displacement relation (Equation (4.2)) and then that expression in the stress-strain relation (equation (4.1)) the stress Green's function at $\mathbf{x}$ due to a concentrated time harmonic force at $\mathbf{y}$ can be obtained. For isotropic, homogeneous, linear elastic material, the stress Green's functions thus obtained are

$$
S_{i j}(\mathbf{x} ; \mathbf{y})=\mu\left(G_{i k, j}+G_{j k, i}\right) P_{k}+\lambda \delta_{i j} G_{k q, k} P_{q}
$$

Superimposing for $M$ point sources the stress in the solid is obtained as,

$$
S_{i j}=\sum_{m=1}^{M}\left[\left(S_{i j}^{1}\right)^{m} P_{1}^{m}+\left(S_{i j}^{2}\right)^{m} P_{2}^{m}+\left(S_{i j}^{3}\right)^{m} P_{3}^{m}\right]=\sum_{m=1}^{M} \mathbf{S}_{i j}{ }^{m}(\mathbf{P})^{m}
$$

Matrix representations of equations (4.6) and (4.8) are

$$
\begin{aligned}
& \mathbf{u} \boldsymbol{i}_{T}=\mathbf{D S i} \boldsymbol{i}_{T S} \cdot \mathbf{A}_{s} \\
& \mathbf{S} \boldsymbol{i}_{T}=\mathbf{S i j} \boldsymbol{j}_{T S} \cdot \mathbf{A}_{s}
\end{aligned}
$$

\subsubsection{Computation of displacement and pressure fields in the fluid}

The pressure field at position $\mathbf{y}$ in the fluid, due to the point sources at position $\mathbf{x}$ distributed over surface $S$, can be expressed as

$$
p(y)=\int_{S} B \frac{\exp \left(i k_{f} r\right)}{4 \pi r} d S(x)
$$

In which $B$ is proportional to the source velocity amplitude.

When the transducer surface is divided into small areas $\Delta S_{m}$ associated with the source points then the above integral can be written in terms of the summation as follows: 


$$
p(y)=\sum_{m=1}^{N}\left(\frac{B}{4 \pi} \Delta S_{m}\right) \frac{\exp \left(i k_{f} r_{m}\right)}{r_{m}}=\sum_{m=1}^{N} A_{m} \frac{\exp \left(i k_{f} r_{m}\right)}{r_{m}}
$$

In which $A_{m}$ is the source strength of the $m$-th point source. Source strengths can be assumed uniform over the transducer face for a uniformly vibrating transducer. $N$ is the total number of point sources, $r_{m}$ is the distance between the $m$-th point source and the target point or the observation point.

Pressure and displacement fields at point $\mathrm{P}$ due to a single point source at a distance $r$ from point $\mathrm{P}$ is given by [26]

$$
\begin{aligned}
& p=\frac{e^{i k_{f} r}}{4 \pi r} \\
& u_{j}=\frac{\left(y_{j}-x_{j}\right)}{4 \pi \rho \omega^{2} r^{2}}\left[i k_{f}-\frac{1}{r}\right] e^{i k_{f} r}
\end{aligned}
$$

Where $p$ is the pressure and $u_{j}$ is the displacement vector; the point source is located at coordinate $x_{j}$ and the coordinate of point $\mathrm{P}$ is $y_{j}$.

Superimposing the contributions of these source points the pressure field for spherical and cylindrical focused lenses have been computed when there is no inhomogeneity in front of the transducer [86]. DPSM has been used for computing the scattered field generated by single spherical cavity in a fluid medium [49] considering two groups of point sources, one group on the boundary of the cavity and the second group on the boundary of the lens. Final results were obtained by superimposing the contributions of these two groups of point source. Then this work was extended to the problem geometry involving multiple cavities in a fluid medium [85]. 

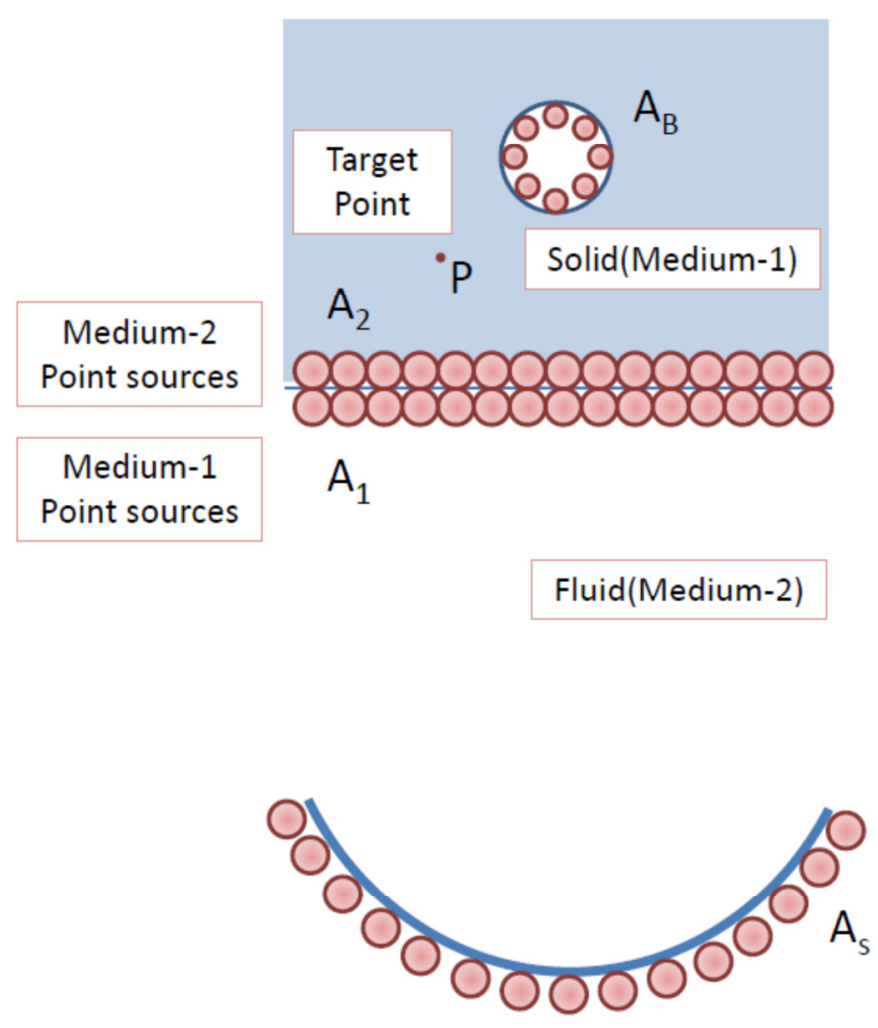

Figure 4.5 (color online) Problem geometry showing the distribution of point sources (small circles) along the acoustic lens (or transducer), the cavity and the interface between the solid and fluid media (see [90]).

\subsubsection{Boundary and Interface Conditions}

To model the ultrasonic field in presence of a cavity in a solid medium, the acoustic lens, the cavity and the fluid-solid interface must be modeled by distributed point sources as shown in Figure 4.5. All boundary and interface conditions must be satisfied simultaneously. The ultrasonic field at a general point P (in the DPSM literature it is known as the target point) is obtained by superimposing the contributions of all point sources that can contribute to that point.

The target point (shown in Figure 4.5) can be placed anywhere - on the lens-fluid interface, the cavity surface or the fluid-solid interface. The pressure and displacement 
fields at these points can be obtained from Equation (4.12) if they are located in the fluid medium, and from equation (4.9) for the solid medium. For point sources with unknown source strengths $\mathbf{A}_{S}, \mathbf{A}_{1}, \mathbf{A}_{2}$ and $\mathbf{A}_{B}$ the pressure, stress and displacement expressions at point $\mathrm{P}$ contain these unknown source strengths. A matrix equation in the following form is obtained by simultaneously satisfying the specified velocity (or displacement) boundary conditions on the lens surface, the continuity conditions on normal and shear stresses and normal displacements at the interfaces, and the traction-free boundary conditions at the cavity surface give [26]

$$
\left[\begin{array}{cccc}
\mathbf{M}_{s s} & \mathbf{M}_{s 2} & 0 & 0 \\
\mathbf{D F} 3_{2 s} & \mathbf{D F} 3_{22} & -\mathbf{D F} 3_{21} & -\mathbf{D F} 3_{2 B} \\
\mathbf{Q}_{2 s} & \mathbf{Q}_{22} & -\mathbf{S} 33_{21} & -\mathbf{S} 33_{2 B} \\
0 & 0 & \mathbf{S} 31_{21} & \mathbf{S} 31_{2 B} \\
0 & 0 & \mathbf{S} 32_{21} & \mathbf{S} 32_{2 B} \\
0 & 0 & \mathbf{S} 33_{B 1} & \mathbf{S} 33_{B B}
\end{array}\right]\left\{\begin{array}{l}
\mathbf{A}_{s} \\
\mathbf{A}_{2} \\
\mathbf{A}_{1} \\
\mathbf{A}_{B}
\end{array}\right\}=\left\{\begin{array}{c}
\mathbf{V}_{s} \\
0 \\
0 \\
0 \\
0 \\
\mathbf{P}_{B}
\end{array}\right\}
$$

If $m 1, n 1, n 2$ and $m 2$ point sources are used to model the lens surface, two layers of source at the interface and the cavity surface then $\mathbf{A}_{S}, \mathbf{A}_{1}, \mathbf{A}_{2}$ and $\mathbf{A}_{B}$ in equation (4.13) should have $m 1, n 1, n 2$ and $m 2$ elements, respectively. Vectors $\mathbf{V}_{S}$ and $\mathbf{P}_{B}$ on the right hand side are obtained from the prescribed boundary conditions. For example, we can assume that the pressures $\mathbf{P}_{B}$ on the surface of the cavity is zero and the surface velocity $\mathbf{V}_{S}$ is uniform on the transducer surface. $\mathbf{V}_{S}$ vector contains the prescribed velocity values at the lens surface at $m 1$ points where small spheres representing the point sources touch the lens surface. Similarly vector $\mathbf{P}_{B}$ contains the pressure values at $m 2$ points on the cavity surface where the point source spheres touch the cavity surface. Matrices $\mathbf{M}, \mathbf{Q}$, 
DF and $\mathbf{S}$ relate the source strengths to the particle velocity, pressure, deformation and normal stress values, respectively. Note that $\mathbf{M}_{S S}, \mathbf{D F} \mathbf{3}_{2 S}, \mathbf{Q}_{2 s,} \mathbf{M}_{s 2}, \mathbf{D F} 3_{22}, \mathbf{Q}_{22}, \mathbf{D F 3}_{21}$, $\mathbf{S 3 3}_{21}, \mathbf{S 3 1}_{21}, \mathbf{S 3 2}_{21}, \mathbf{S 3 3}_{\mathrm{B} 1}, \mathbf{D F}_{2 B}, \quad \mathbf{S 3 3}_{2 B}, \mathbf{S 3 1}_{2 B}, \mathbf{S 3 2}_{2 B}$ and $\mathbf{S 3 3}_{B B}$ matrices have dimensions $m 1 \times m 1, n 2 \times m 1, n 2 \times m 1, m 1 \times n 2, n 2 \times n 2, n 2 \times n 1, n 2 \times n 1, n 2 \times n 1, n 2 \times n 1, m 2 \times n 1$, $n 2 \times m 2, n 2 \times m 2$ and $m 2 \times m 2$, respectively. To obtain the source strengths the system of equations given in equation (4.13) are to be solved.

In this case if the resultant velocity on the transducer surface is specified as $\left\{\mathbf{V}_{\mathrm{S}}\right\}$ and the pressure on the cavity surface is zero then the source strengths are obtained from the following system of equations

$$
\left[\begin{array}{cccc}
\mathbf{M}_{s s} & \mathbf{M}_{s 2} & 0 & 0 \\
\mathbf{D F} 3_{2 s} & \mathbf{D F} 3_{22} & -\mathbf{D F} 3_{21} & -\mathbf{D F} 3_{2 B} \\
\mathbf{Q}_{2 s} & \mathbf{Q}_{22} & -\mathbf{S} 33_{21} & -\mathbf{S} 33_{2 B} \\
0 & 0 & \mathbf{S} 31_{21} & \mathbf{S} 31_{2 B} \\
0 & 0 & \mathbf{S} 32_{21} & \mathbf{S} 32_{2 B} \\
0 & 0 & \mathbf{S} 33_{B 1} & \mathbf{S} 33_{B B}
\end{array}\right]\left\{\begin{array}{c}
\mathbf{A}_{s} \\
\mathbf{A}_{2} \\
\mathbf{A}_{1} \\
\mathbf{A}_{B}
\end{array}\right\}=\left\{\begin{array}{c}
\mathbf{V}_{s} \\
0 \\
0 \\
0 \\
0 \\
0
\end{array}\right\}
$$

However, if the pressure [22] on the transducer surface instead of the velocity is specified then the source strengths are obtained from the following equations

$$
\left[\begin{array}{cccc}
\mathbf{Q}_{s s} & \mathbf{Q}_{s 2} & 0 & 0 \\
\mathbf{D F} 3_{2 s} & \mathbf{D F} 3_{22} & -\mathbf{D F} 3_{21} & -\mathbf{D F} 3_{2 B} \\
\mathbf{Q}_{2 s} & \mathbf{Q}_{22} & -\mathbf{S} 33_{21} & -\mathbf{S} 33_{2 B} \\
0 & 0 & \mathbf{S} 31_{21} & \mathbf{S} 31_{2 B} \\
0 & 0 & \mathbf{S} 32_{21} & \mathbf{S} 32_{2 B} \\
0 & 0 & \mathbf{S} 33_{B 1} & \mathbf{S} 33_{B B}
\end{array}\right]\left\{\begin{array}{l}
\mathbf{A}_{s} \\
\mathbf{A}_{2} \\
\mathbf{A}_{1} \\
\mathbf{A}_{B}
\end{array}\right\}=\left\{\begin{array}{c}
\mathbf{P}_{s} \\
0 \\
0 \\
0 \\
0 \\
0
\end{array}\right\}
$$

Both equations (4.14) and (4.15) require solution of a system of linear equations. This solution is valid for any size of cavity placed at any location. 
The number of cavities can be increased in the model if necessary. If there are two cavities in the solid medium, the second cavity surface should have an additional layer of point sources. If the source strength vectors for the two cavities are $\mathbf{A}_{B 1}$ and $\mathbf{A}_{B 2}$ then the matrix equation would be as follows:

$$
\left[\begin{array}{ccccc}
\mathbf{M}_{s s} & \mathbf{M}_{s 2} & 0 & 0 & 0 \\
\mathbf{D F} 3_{2 s} & \mathbf{D F} 3_{22} & -\mathbf{D F} 3_{21} & -\mathbf{D F} 3_{2 B 1} & -\mathbf{D F} 3_{2 B 2} \\
\mathbf{Q}_{2 s} & \mathbf{Q}_{22} & -\mathbf{S} 33_{21} & -\mathbf{S} 33_{2 B 1} & -\mathbf{S} 33_{2 B 2} \\
0 & 0 & \mathbf{S} 31_{21} & \mathbf{S} 31_{2 B 1} & \mathbf{S} 31_{2 B 2} \\
0 & 0 & \mathbf{S} 32_{21} & \mathbf{S} 32_{2 B 1} & \mathbf{S} 32_{2 B 2} \\
0 & 0 & \mathbf{S} 33_{B 11} & \mathbf{S} 33_{B I B 1} & \mathbf{S} 33_{B I B 2} \\
0 & 0 & \mathbf{S} 33_{B 21} & \mathbf{S} 33_{B 2 B 1} & \mathbf{S} 33_{B 2 B 2}
\end{array}\right]\left\{\begin{array}{c}
\mathbf{A}_{s} \\
\mathbf{A}_{2} \\
\mathbf{A}_{I} \\
\mathbf{A}_{B I} \\
\mathbf{A}_{B 2}
\end{array}\right\}=\left\{\begin{array}{c}
\mathbf{V}_{s} \\
0 \\
0 \\
0 \\
0
\end{array}\right\}
$$

Equation (4.16) is also valid for any size and location of the cavities with respect to each other and the solid-fluid interface.

\subsection{Numerical Results}

Following the above formulation, the ultrasonic field in front of an acoustic lens (or transducer) submerged in a fluid adjacent to a solid half-space is modeled. The solid half-space contains single or multiple cavities. When there is only one cavity in the solid medium the ultrasonic field is computed for different locations of the cavity with respect to the focal point of the lens and the solid/fluid interface to investigate at what locations the cavity is detectable by the acoustic lens and at what locations it is not. The computed field for the single cavity case is compared with that for the multiple cavities to see if the focused lens is capable of distinguishing single cavity from two cavities in close proximity. Material properties for the fluid and solid media used in our computations are 
given as: (Water: $\rho=1.0 \mathrm{~g} / \mathrm{cm}^{3}, \mathrm{C}_{\mathrm{p}}=1.5 \mathrm{~km} / \mathrm{s}$; Plexiglas: $\rho=1.15 \mathrm{~g} / \mathrm{cm}^{3}, \mathrm{C}_{\mathrm{p}}=2.7 \mathrm{~km} / \mathrm{s}, \mathrm{C}_{\mathrm{s}}$ $=1.1 \mathrm{~km} / \mathrm{s}$; Glass-Quartz: $\left.\rho=2.2 \mathrm{~g} / \mathrm{cm}^{3}, C_{p}=5.57 \mathrm{~km} / \mathrm{s}, C_{\mathrm{s}}=3.43 \mathrm{~km} / \mathrm{s}\right)$. The concave lens and the cavity locations relative to the fluid-solid interface are shown in Figure 4.5. The opening angle of the transducer (also known as the lens angle) and the lens radius are $100^{\circ}$ and $20 \mathrm{~mm}$, respectively. The transducer is excited at $1 \mathrm{MHz}$ frequency. Note that modeling this large lens at $1 \mathrm{MHz}$ frequency is equivalent to modeling a much smaller lens, say $50 \mu \mathrm{m}$ radius with $100^{\circ}$ lens opening angle, operating at $400 \mathrm{MHz}$ frequency because in these two cases the lens radius/wavelength ratio is the same. The lens surface is modeled by 4003 point sources, distributed slightly behind the lens face as shown in Figure 4.5. The number of point sources needed is decided based on the convergence criterion [26] - the spacing between two neighboring point sources is less than one-third wavelength.

For cavity detection by the reflection mode acoustic microscopy the acoustic lens generates the ultrasonic beam as well as receives the reflected signal from the cavity. The pressure field generated by the reflected ultrasonic energy on the lens face is integrated to compute the acoustic force sensed by the lens due to the reflected signal. This force is compared with the input energy as was done by Placko et al. [49] for the single cavity detection in a fluid. They have shown that the acoustic force (or the integration of the pressure on the lens surface) varies noticeably as the cavity in the fluid is moved from the focal point to the right, left, up or down. The same method is used here to show the change in the acoustic force when the cavity in the solid moves relative to the focal point of the lens. 
Lobkis et al. [48] presented an approximate theoretical analysis to compute the reflected beam strength. Figure 4.6 highlights the limitation of this approximate analytical solution. This figure shows a comparison between the approximate analytical solution and the DPSM prediction for a single cavity in a Plexiglas half-space immersed in water. The horizontal axis shows the distance between the center of the cavity and the focal point multiplied by the wave number $\mathrm{k}$ of the liquid. The vertical axis plots the reflected force or the integration of the acoustic pressure generated by the reflected ultrasonic energy on the lens surface. One noticeable difference between these two curves is the presence of multiple oscillations in the DPSM curve but not in the simplified theoretical curve. Oscillations arise from the multiply reflected beams between the lens and the fluid-solid interface, as well as that between the cavity and the interface. Analytical solution completely ignores that. Another shortcoming of the analytical solution is that it assumes that the wave reflected at the liquid/solid interface is not affected by the presence of the cavity. In other words, the waves scattered by the cavity are assumed to have negligible contribution to the Rayleigh wave generation at the liquid-solid interface. This assumption is acceptable for a cavity smaller than the wavelength of the Rayleigh wave. A third shortcoming of the theoretical analysis is that the reflected force is integrated on the entire interface between the fluid and solid halfspaces instead of integrating it on the lens surface only that makes the analytical result less realistic. In the DPSM analysis none of these simplifying assumptions are necessary. Figure 4.6 demonstrates that all these simplifying assumptions make the theoretical results significantly different from the real solution. 


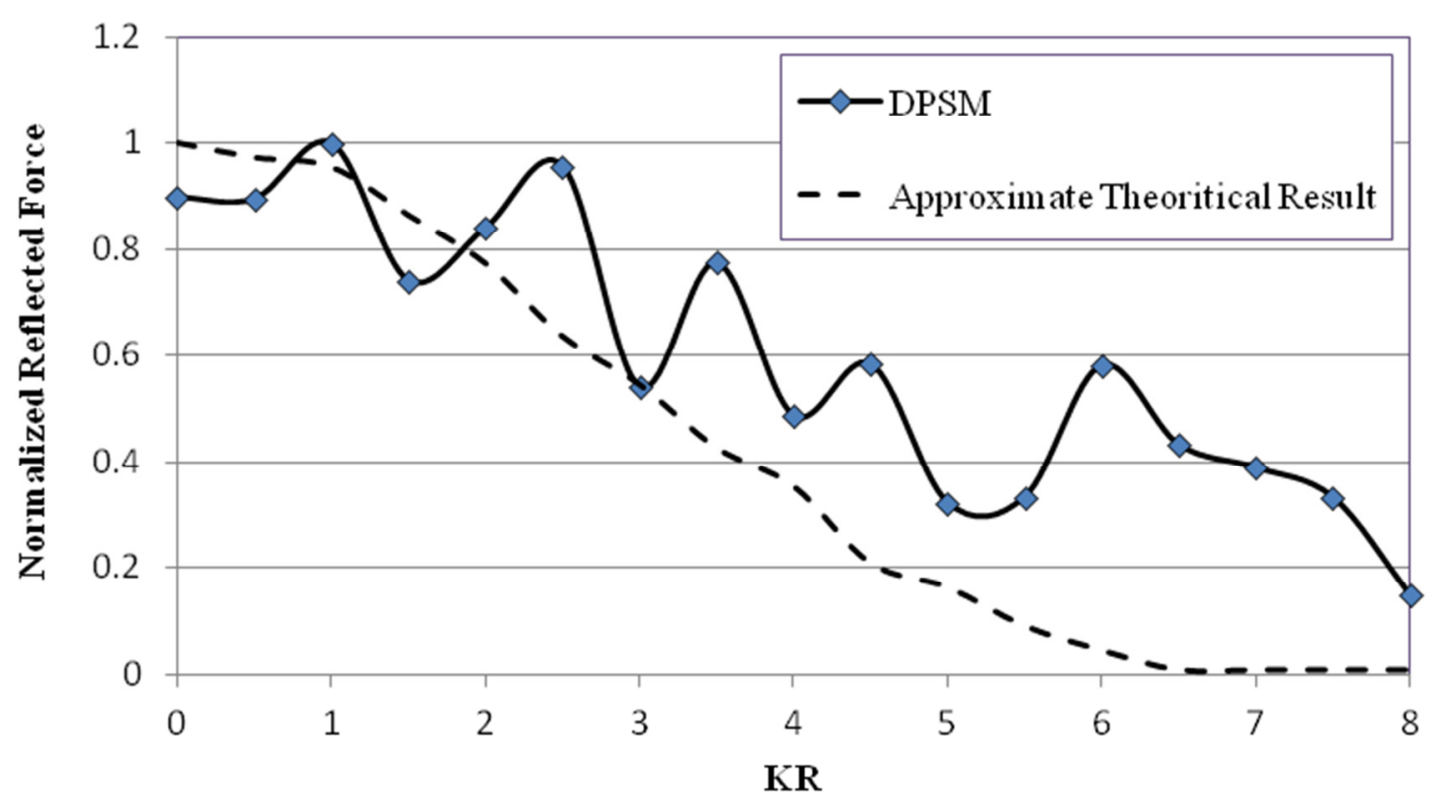

Figure 4.6 Comparison between the DPSM results with the approximate theoretical solution for a cavity in Plexiglas (radius of the cavity $=0.16 \mathrm{~mm}$, cavity center from the solid-fluid interface $=\mathbf{4 . 8} \mathbf{m m}$, radius of the lens $=2 \mathrm{~cm}$, lens angle $=60^{\circ}$, Freq $\left.=1.5 \mathrm{MHz}\right)($ see $[90])$.

Figure 4.7 shows the normalized force felt by the acoustic lens when the ultrasonic beam is reflected by the single cavity present in the solid medium (GlassQuartz), as a function of the horizontal distance of the cavity from the focal point. In Figure 4.7 the cavity radius is $0.2 \mathrm{~mm}$, the distance of the cavity center from the solidfluid interface is $0.5 \mathrm{~mm}$ and the center of curvature of the lens (denoted as the lens center) is inside the solid at a distance $0.3 \mathrm{~mm}$ from the solid-fluid interface. The horizontal axis in Figure 4.7 shows the horizontal distance between the cavity center and the lens center. As the cavity moves towards right or left from the central axis of the lens this distance increases. One can see in this figure that the presence of the cavity is clearly sensed by the lens as the reflected force (force felt by the acoustic lens due to the reflected ultrasonic energy) is reduced by $8 \%$ when the lens is above the cavity position. 
It should be noted that for the horizontal distance of the cavity center from the lens center between 0 and $6 \mathrm{~mm}$ the acoustic lens is most sensitive to the presence of the cavity, while it cannot sense the cavity when this distance exceeds $15 \mathrm{~mm}$.

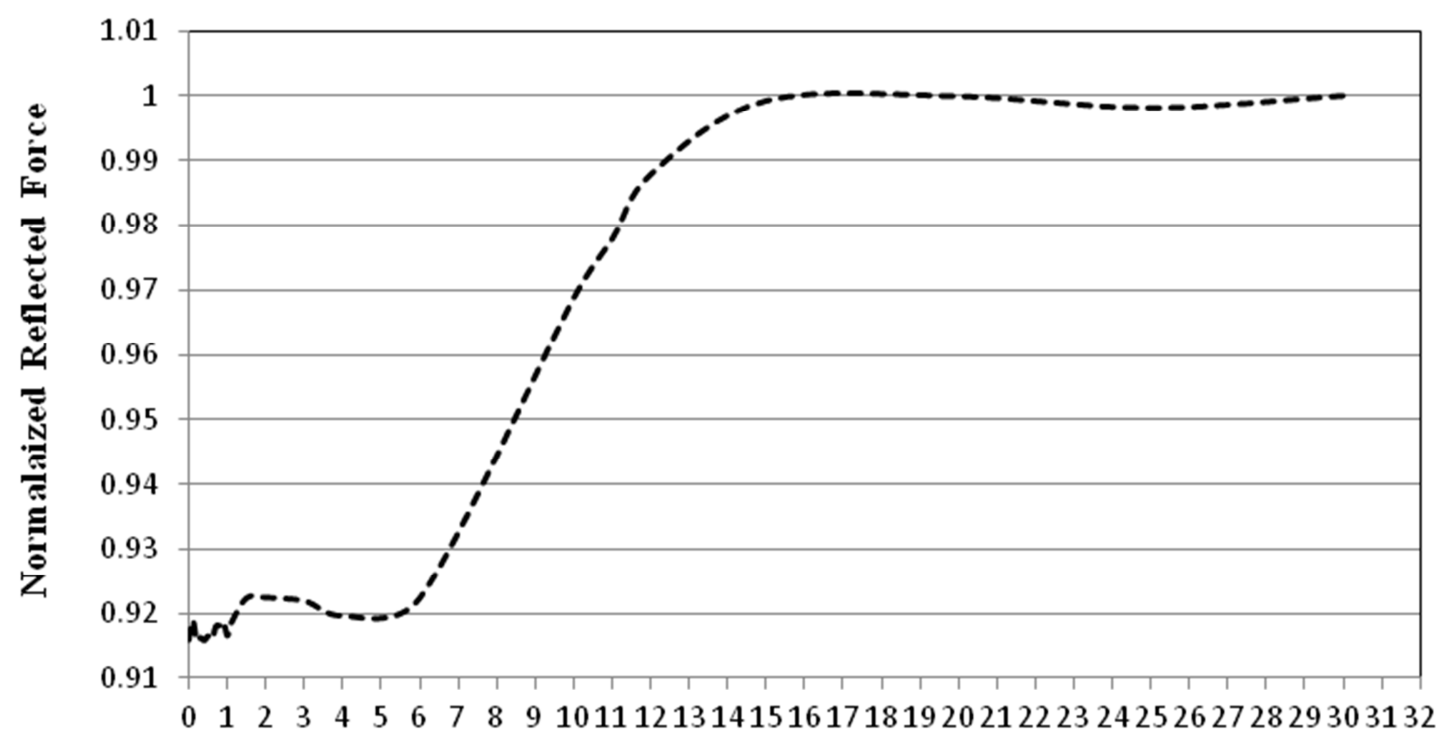

Horizontal distance between the lens center and the center of the cavity (mm)

Figure 4.7 Reflected force (force felt by the acoustic lens due to the reflected ultrasonic signal) versus the horizontal distance between the lens center (the center of curvature of the lens) and the cavity center when there is only one cavity of radius $0.2 \mathrm{~mm}$ present in the solid medium (Glass-Quartz). Distance of the cavity center from the fluid-solid interface $=0.5 \mathrm{~mm}$, the center of curvature of the lens is at a depth of $0.3 \mathrm{~mm}$ inside the solid (see [90]).

Figure 4.8 shows similar results as Figure 4.7; the only difference is that the distance of the lens center from the solid-fluid interface is now increased to $0.5 \mathrm{~mm}$, meaning the lens is moved closer to the interface. Figure 4.8 shows the acoustic lens having the highest sensitivity to the cavity when the horizontal distance between the cavity center and the lens center is less than $5 \mathrm{~mm}$ while it is almost insensitive to the 
cavity as this distance becomes greater than $11 \mathrm{~mm}$. In this case the reflected force is reduced by only $2.2 \%$ as opposed to $8 \%$ in Figure 4.7 .

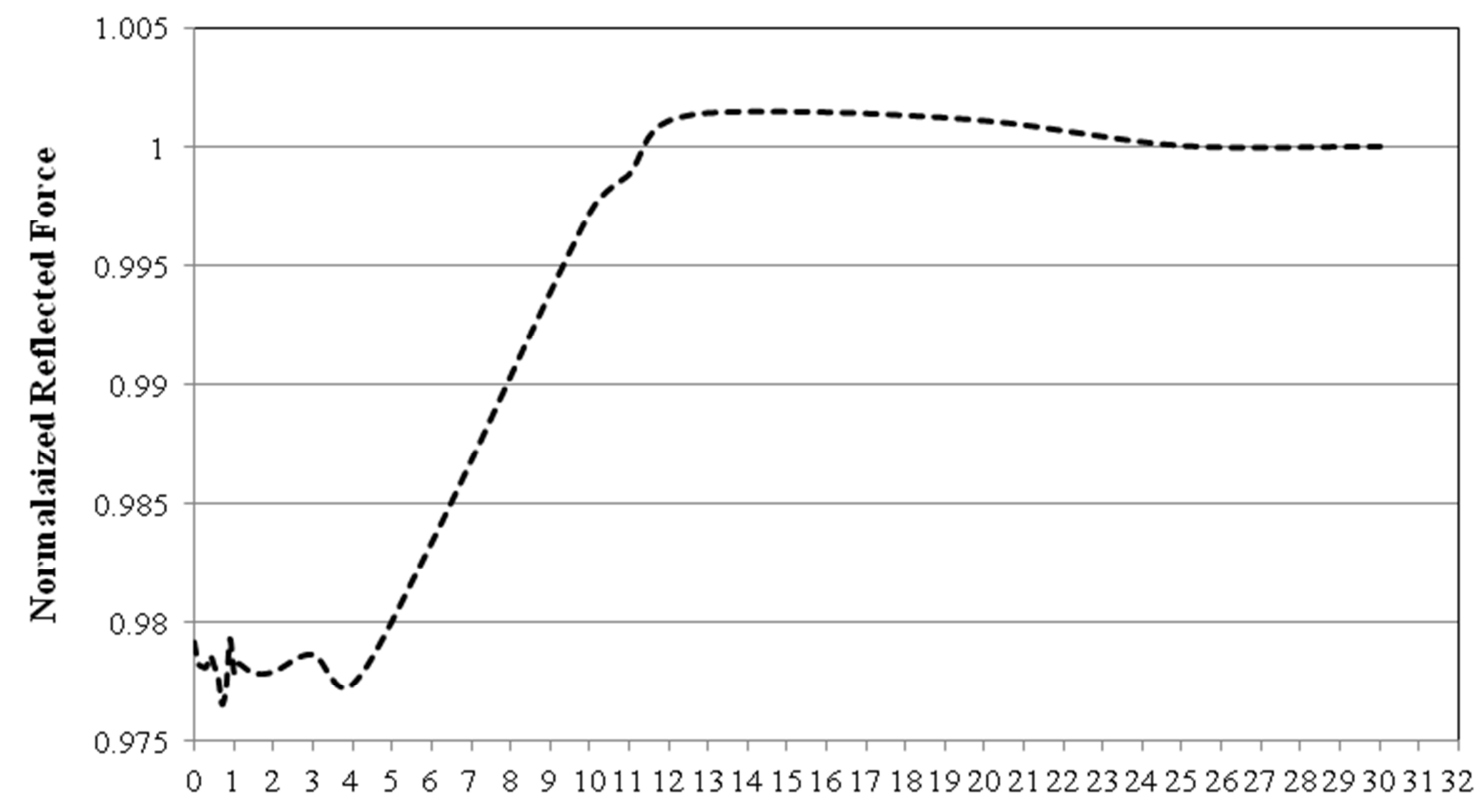

Horizontal distance between the lens center and the center of the cavity (mm)

Figure 4.8 Reflected energy generated force vs. the horizontal distance between the lens center (the center of curvature of the lens) and the cavity center when there is only one cavity of radius $0.2 \mathrm{~mm}$ present in the solid medium. Distance of the cavity center from the fluid-solid interface $=0.5 \mathrm{~mm}$, the lens center is at a depth of 0.5 mm inside the solid (see [90]).

Figure 4.9 shows the effect of the cavity size on the normalized reflected force. The solid line is for a cavity of radius $0.2 \mathrm{~mm}$ while the dashed line is for a cavity of radius $0.3 \mathrm{~mm}$. Figure 4.9 clearly shows that the radius of the cavity in the solid medium affects the reflected force - as the radius increases from $0.2 \mathrm{~mm}$ to $0.3 \mathrm{~mm}$ the reflected force increases significantly. Therefore, if the detecting device is properly calibrated then the cavity size can be estimated from the reflected force measurement. 
Figure 4.6 to Figure 4.9 show that the presence of a cavity in a solid half-space can be detected by a focused lens immersed in a fluid half-space. Horizontal location of the cavity can be easily predicted by identifying the horizontal location of the lens where the reflected energy felt by the lens deviates most from the surrounding region. It should be noted that depending on the distance of the cavity and the lens from the fluid-solid interface the force felt by the lens due to the reflected energy from the cavity can be greater (Figure 4.6 and Figure 4.9) or smaller (Figure 4.7 and Figure 4.8) as the central axis of the lens is aligned with the cavity center.

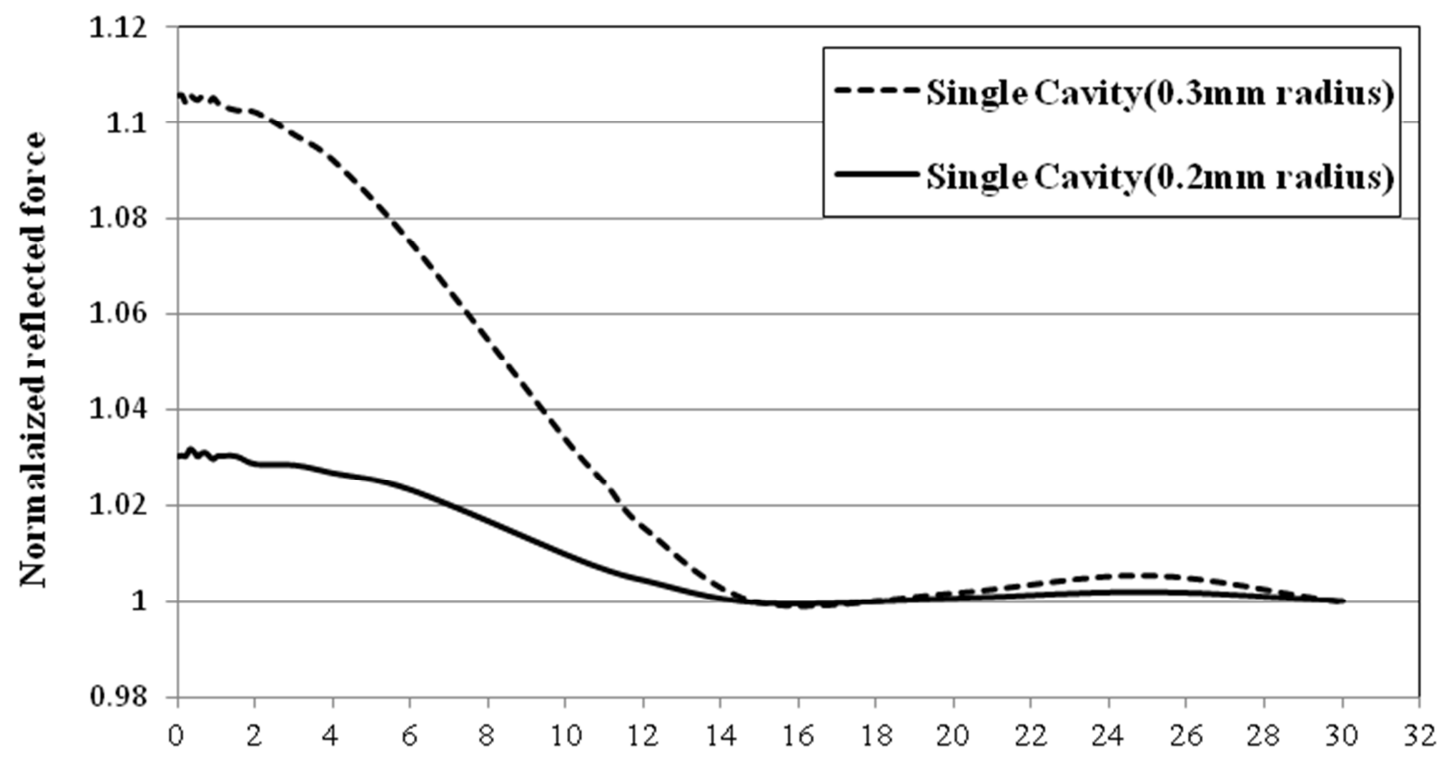

Horizontal distance between the lens center and the center of the cavity

Figure 4.9 Reflected energy generated force as a function of the horizontal distance between the lens center and the cavity center when there is only one cavity present in the solid medium. Cavity radius is $0.2 \mathrm{~mm}$ (solid line) and $0.3 \mathrm{~mm}$ (dashed line), cavity center distance from the fluid-solid interface is $\mathbf{1} \mathbf{~ m m}$, distance of the lens center (the center of curvature of the lens) from the interface is $0.5 \mathrm{~mm}$. High oscillations near the peaks of the curve are noted. Figure 4.11 is plotted to investigate this high oscillation phenomenon more closely (see [90]). 
In order to see if the focused lens is capable of distinguishing between two cavities in close proximity, reflected ultrasonic energy from single and double cavities are compared in Figure 4.10. For the problem geometry with two cavities the horizontal distance of the lens center is measured from the midpoint of the two cavities while for the single cavity case it is measured from the center of the cavity. For two cavities in close proximity (with $0.5 \mathrm{~mm}$ and $1 \mathrm{~mm}$ spacing in Figure 4.10) the force plot shows one dominant peak, as if the energy is reflected from one big cavity. However, when the distance between the two cavity centers is increased to $3 \mathrm{~mm}$ two distinct peaks separated by $3 \mathrm{~mm}$ appear. Therefore, in this case the cavity spacing can be predicted from the distance between the two neighboring peaks in the force vs. horizontal distance plot.

Figure 4.11 shows the reflected force for a number of different geometries - when there is only one cavity of radius $0.2 \mathrm{~mm}$ present in the solid half-space and when two cavities with the cavity spacing varying between $0.5 \mathrm{~mm}$ and $6 \mathrm{~mm}$. It is interesting to note that in all curves high oscillations occur when the lens is placed right above the cavity center and again when it is at the central position right above the midpoint of the two cavities in close proximity. These oscillations probably occur due to multiple reflections of the normally incident acoustic energy between the fluid-solid interface and the cavity. When two cavities are in close proximity then the two cavities behave almost like one big cavity and the multiple reflection phenomenon is observed at the central position in addition to the locations right above the cavities. As the distance between the cavities is increased well-separated two peaks start to appear and high oscillations are observed only for the lens positions just above the cavities. 


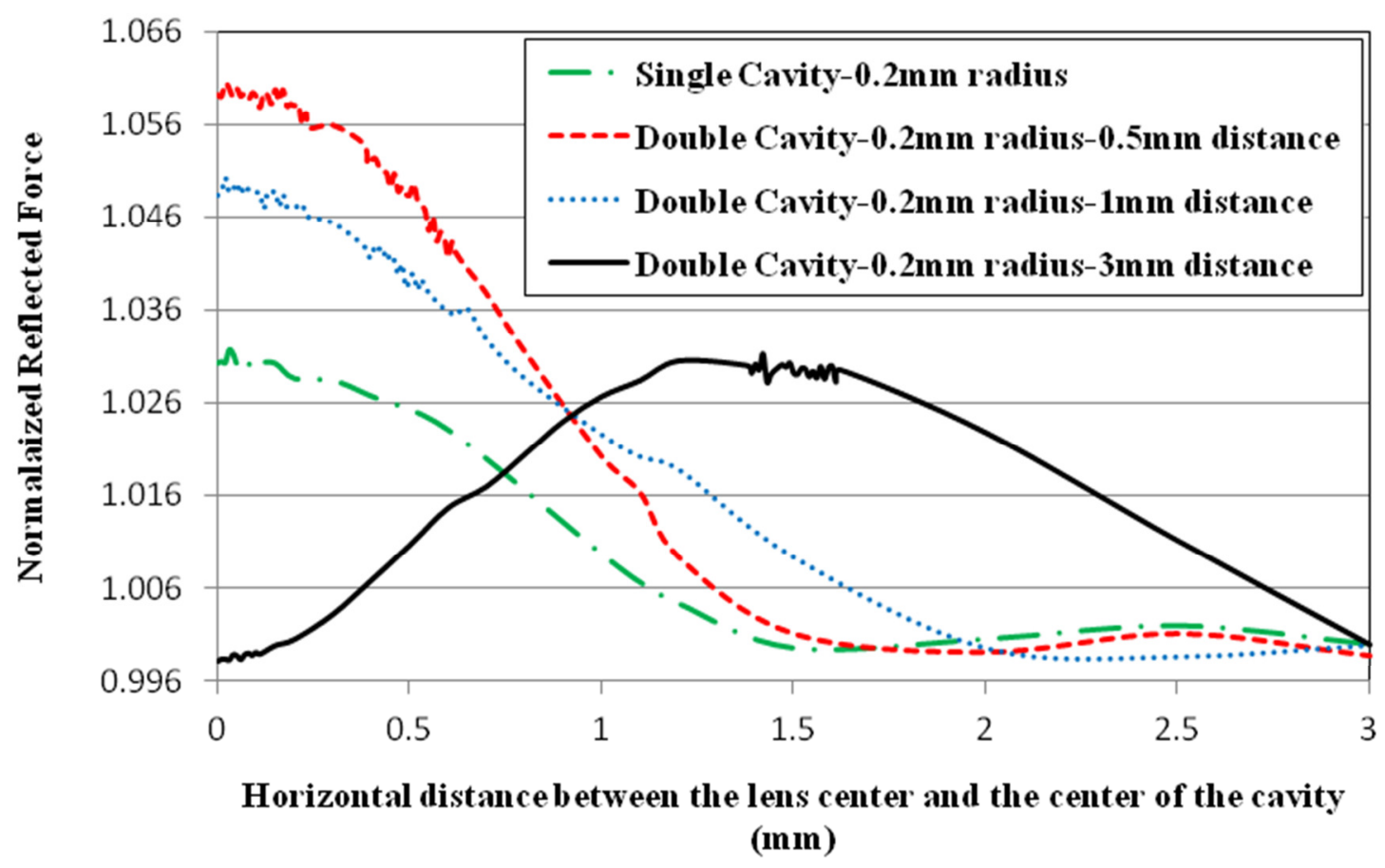

Figure 4.10 Reflected energy generated force as a function of the horizontal distance of the lens center, from the cavity center (for the single cavity) or from the midpoint of the two cavities (for the double cavities), distance of the fluid-solid interface is $1 \mathrm{~mm}$ from the cavity center and $\mathbf{0 ~} \mathrm{mm}$ from the lens center. High oscillations near the peaks in some curves are noted. Figure 4.11 is plotted to investigate this high oscillation phenomenon more closely (see [90]).

In Figure 4.11 from the single cavity response one can see that noticeable reflected force from the cavity is felt over a $3 \mathrm{~mm}$ horizontal distance $(1.5 \mathrm{~mm}$ on each side of the cavity center) while some small but non-zero influence is felt over $6 \mathrm{~mm}$ horizontal distance (3 $\mathrm{mm}$ on each side of the cavity). As a result, when the cavity centers are separated by less than $3 \mathrm{~mm}$ then the influence zones of the two cavities overlap giving rise to a response that looks like a single cavity response. When the cavity spacing exceeds $3 \mathrm{~mm}$ then the influence of the second cavity is significantly reduced and the reflected force plot shows two distinct well-separated hills appear at the locations of the two cavities. 
The main difference between Figure 4.10 and Figure 4.11 is in the distance between the lens center and the fluid-solid interface. In Figure 4.10 this distance is zero and the single cavity produces only one maximum in the reflected force curve. In Figure 4.11 this distance is changed to $0.5 \mathrm{~mm}$ which creates two maxima of the reflected force curve at $+/-0.5 \mathrm{~mm}$ even for the single cavity case.

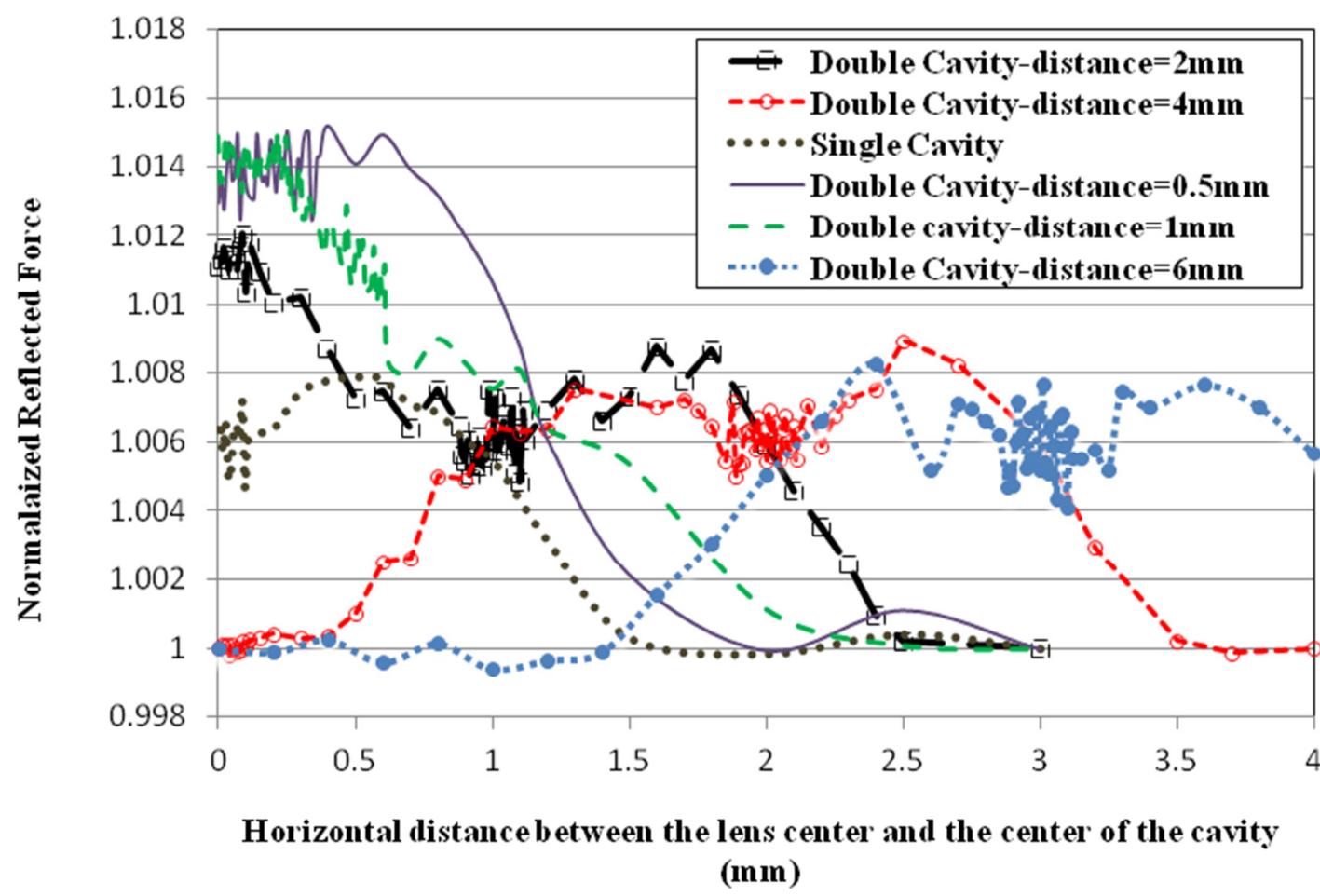

Figure 4.11 Reflected force vs. the horizontal distance of the lens center, from the cavity center (for the single cavity case) or from the midpoint of the two cavities (for the double cavity case), distance of the fluid-solid interface from the cavity center is $1 \mathrm{~mm}$ and from the lens center it is $0.5 \mathrm{~mm}$, cavity radius $=0.2 \mathrm{~mm}$ (see [90]).

\subsection{Conclusions}

Ultrasonic fields in front of a point focused lens immersed in a fluid medium adjacent to a solid half-space containing single and multiple cavities are computed by semi-analytical DPSM formulation. The results are obtained without ignoring any 
interaction effect. The main difference between the analysis involving cavities in fluid media available in the literature and the analysis presented here is that the presence of the solid medium makes the problem more challenging. The interaction between the cavities and the interface affects the solution. To correctly model this interaction effect the entire problem - the interface, the lens surface and the cavities should be considered concurrently in the model; all boundary conditions on the lens and cavity surfaces and continuity conditions across the interface must be satisfied simultaneously, as done here. This analysis shows that a fluctuation of the reflected signal strength indicates the presence of a cavity in the solid in front of the lens. The reflected energy from the cavity may produce higher or lower force at the lens surface when the cavity is detected. The effect of adding a second cavity next to the first one is also studied. When the distance between the cavities is relatively large then the lens can distinctly detect each cavity otherwise reflections from the two cavities in close proximity appear to be the reflection from a bigger cavity. 


\section{CHAPTER 5}

\section{DEVELOPMENT OF THE FORTRAN DPSM3D CODE}

\subsection{DPSM3D Code}

In order to solve DPSM problems discussed in previous chapters a FORTRAN code is developed. It is designed in a general form to make it capable of modeling various types of geometries in a 3D-space (see Figure 5.1). Point sources are distributed over the boundaries and interfaces as needed by the DPSM. The loading (which can be a Terahertz Gaussian Beam source or an ultrasonic transducer) is applied and then the problem is solved to find the point source strengths. After getting the point source strengths the post-processing job of plotting the desired field variables for a user defined plot-section or plot-line is carried out. The main idea of the new code is modeling the whole domain as a combination of some interface surfaces. Each interface is the boundary between two neighboring media and putting two point source groups on two sides of the interface mimics the two-medium interactions satisfying the compatibility conditions.

An object-oriented approach is followed to define each interface as a FORTRAN Type-statement. In the current code we can define many types of interface such as 4sided polygon, circle, sphere and hemi-spheres as shown in Figure 5.2. Complex geometries can be modeled combining the above mentioned simple objects. The code is written in a general way such that any extra interface type (like Bezier surfaces) can be added in the future. 


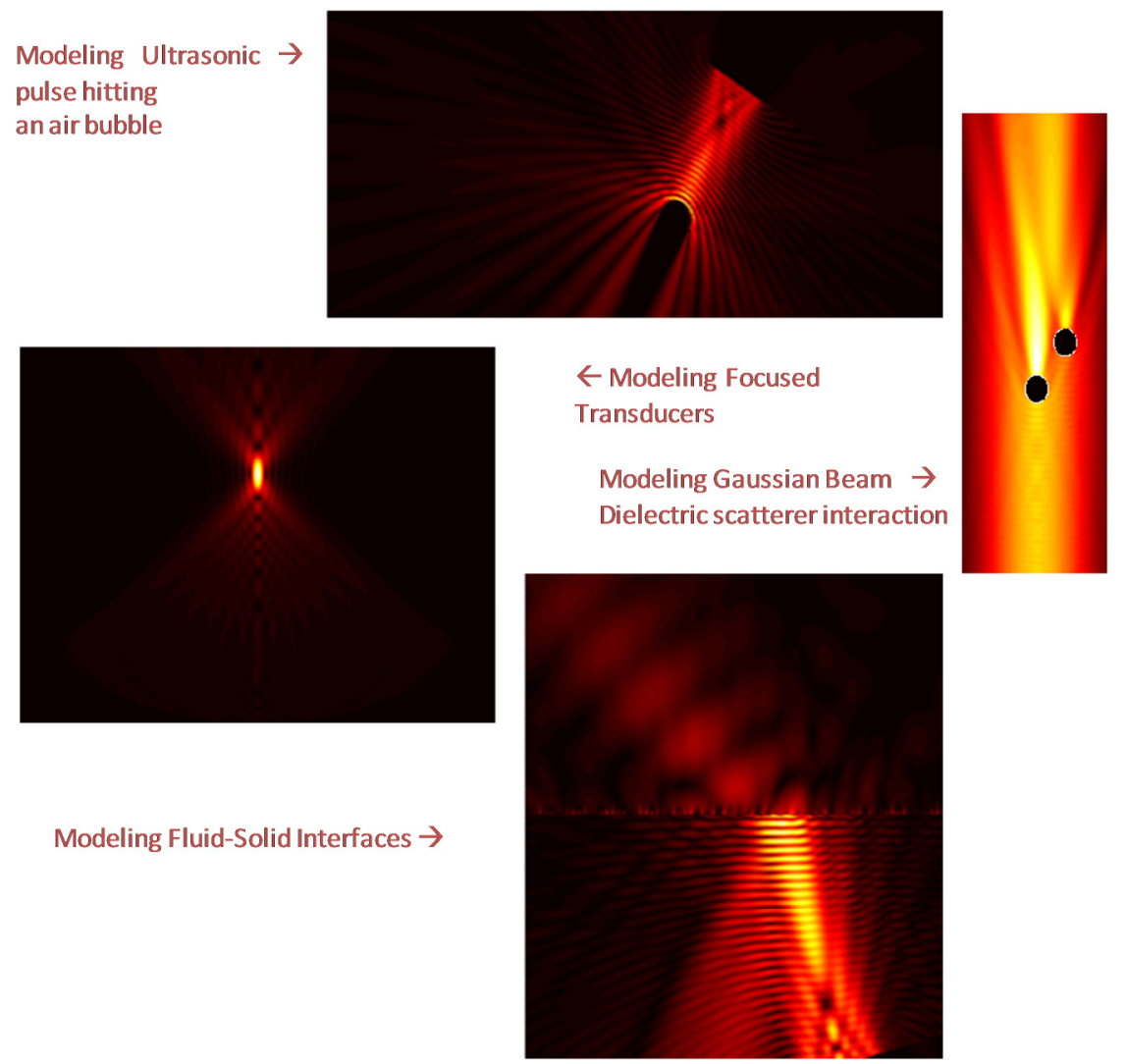

Figure 5.1 Different types of problem analyzed by DPSM3D code

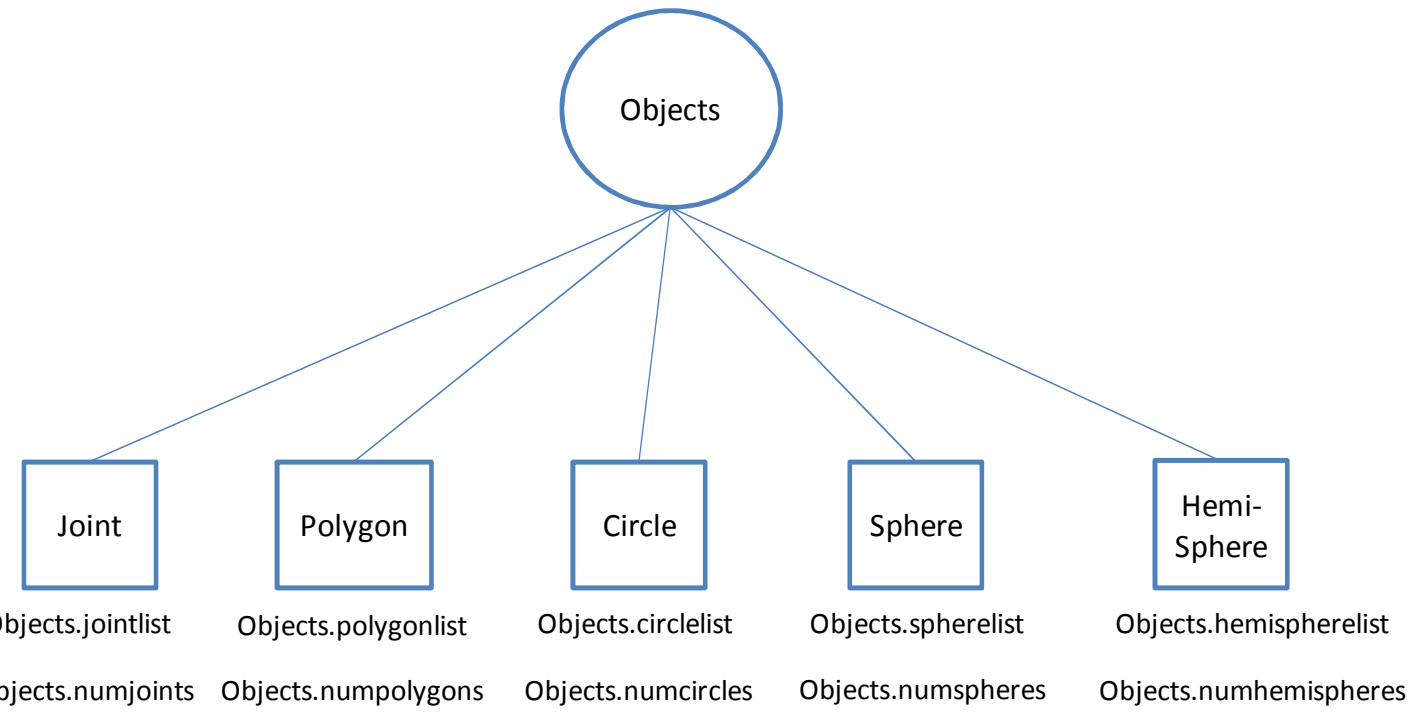

Figure 5.2 The objects oriented structure of interfaces in DPSM3D 
Figure 5.3 shows the FORTRAN scripts that should be put in the main body of the code to define a Polygon interface object which is an interface between mediumID $=1$ and mediumID=6.

As a schematic example Figure 5.4 depicts a solid aluminum box inside water, this box is made of 6-interfaces each one separates the aluminum and water media. The ultrasonic transducer is also 'modeled as a circular interface. DPSM3D takes care of assembling the interface objects, distributing point sources on its boundaries, creating the stiffness matrix based on boundary and compatibility conditions, and finally solving for point source strengths.

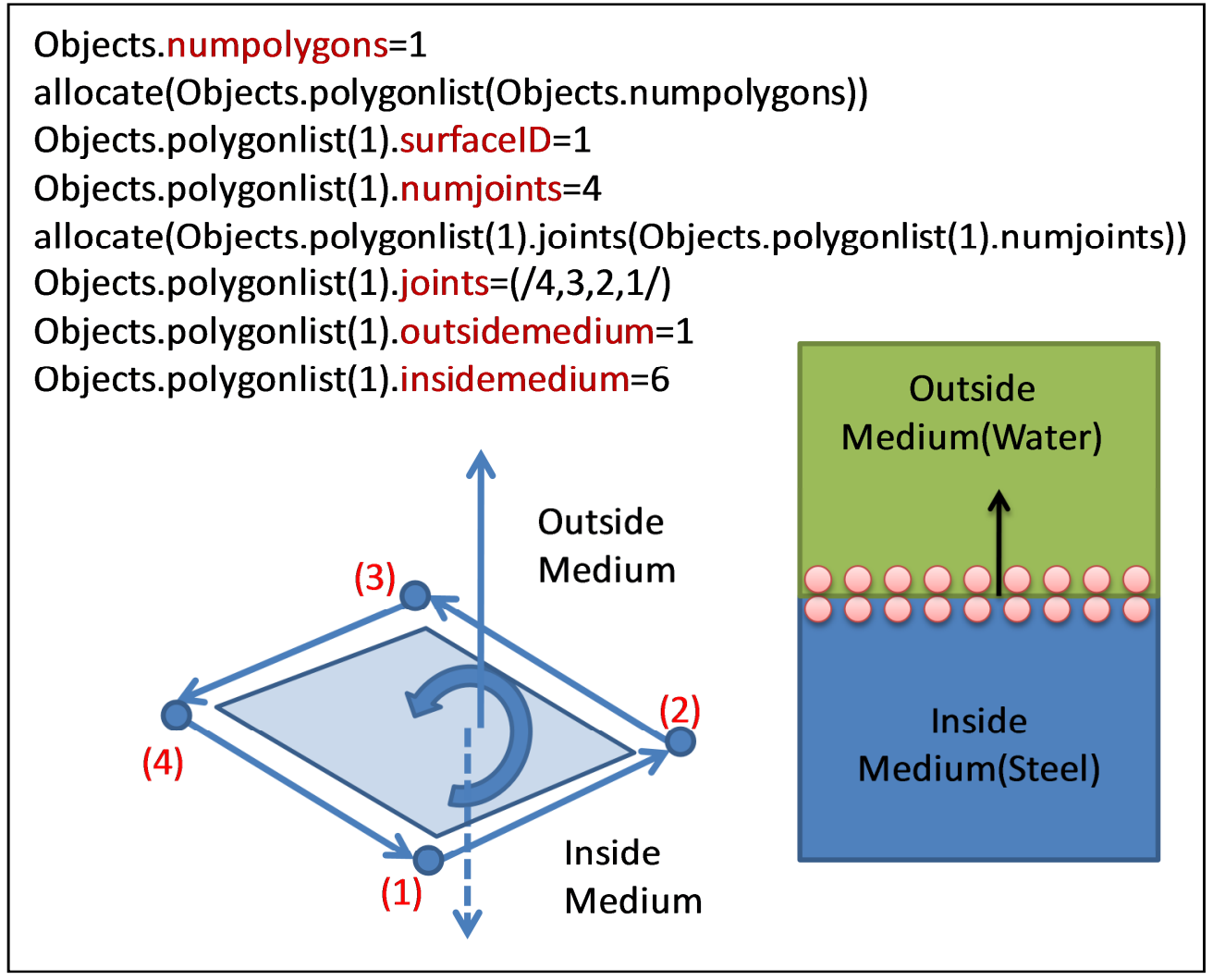

Figure 5.3 Defining a Polygon interface object in DPSM3D 


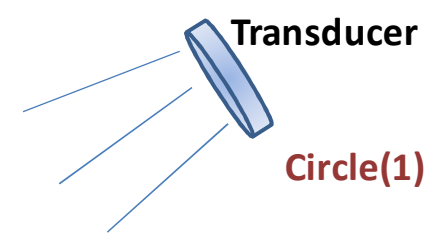

Water

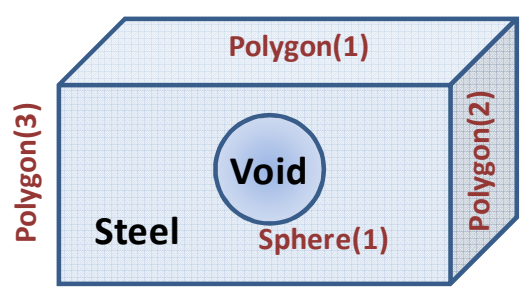

Figure 5.4 Schematic of a DPSM model composed of interface objects

The University of Arizona NDT laboratory research group decided to do the coding in Intel® Visual FORTRAN Compiler environment because of its versatility in doing arithmetic operations. The other reason, nowadays the software developers try to use the pre-written subroutines and packages for their own purpose and many available packages are released in FORTRAN language. Furthermore FORTRAN is a globally accepted language for engineering applications. In the past, the group researchers were using MATLAB codes to solve their problems but when one tries to solve more intensive problems with large amount of point sources, the way MATLAB handles memory is not efficient for large matrices. In the new code this shortcoming is avoided by using algorithms that use "out-of-core" solvers and use hard-disk as the data storage source instead of RAM.

In Appendix A the FORTRAN source code to solve ultrasonic problems is listed. The appendix code geometry inputs have been prepared to model the problem of the 
scattering of focused ultrasonic beams by a cavity in a solid as discussed in detail in CHAPTER 4. Other problems can be easily modeled by changing the input geometric information.

In Appendix B the FORTRAN source code to solve electromagnetic problems is listed. The appendix code geometry inputs have been prepared to model scattering of Gaussian $\mathrm{THz}$ beams by dielectric scatterers as discussed in detail in section 1.7.5 of CHAPTER 1. Other problems can be easily modeled by changing the input geometric information.

Following Appendix A, DPSM3D code is presented. It consists of a main program "DPSM_3d_ultrasonics". In the main body the frequency is defined by assigning desired frequency to variable "Problemomega". Material (medium) properties should be assigned to variable list "Mediumlist". The next step is defining interfaces using objects in Figure 5.2. After defining geomtry, each object should be meshed (point sources distributed over the surface boundaries) using the subroutines specific to each interface type. The meshing subroutines are "Circlemesh", "Spheremesh", "Polygonmesh" and "Hemispheremesh". Finally subtoutines "Plotline" and "PlotSection" in the postprocessing section should be implemented to compute the desired outputs in the user-defined ASCI files. The main program calls (uses) libraries (modules) of "Variables", "geometrylibrary", "meshing", "greensfunctions", "DPSMstructure" and "Excitation".

Using a FORTRAN compiler the above mentioned modules should be compiled and linked to the main program. A commercial package Microsoft Visual Studio that supports 
"Intel Fortran" can be used to run this code or most of the HPC (high performance computing systems) clusters have ready to use FORTRAN compilers to run the program. "ME42" subroutines are parts of the previously developed HSL package (available for free for academic/non-commercial applications) developed by (Rutherford Appleton Laboratory) to solve Sparse-Matrices in an efficient manner. In order to avoid Copyright violation “ME42” HSL solver's source code is not listed here.

\subsection{Case Studies Carried Out by DPSM3D Code}

All DPSM analyses presented in previous chapters have been carried out using DPSM3D code. Validity of this code has been verified by comparing the acquired results with analytical solutions or other globally accepted methods such as FEM as discussed in detail in previous chapters.

In the collaborative research with Hajzargarbashi et. al. [85] DPSM3D has been used to study scattering of focused ultrasonic beams by single or double spherical cavities in a fluid. Figure 5.5 depicts the pressure field in front of an acoustic lens in presence of two small cavities of $0.1 \mathrm{~mm}$ radius. Figure 5.6 shows the normalized force sensed by the acoustic lens when the cavities move horizontally on the focal plane when the signal frequency is $1 \mathrm{MHz}$ and the distance between the cavity centers is $1.2 \mathrm{~mm}$. 


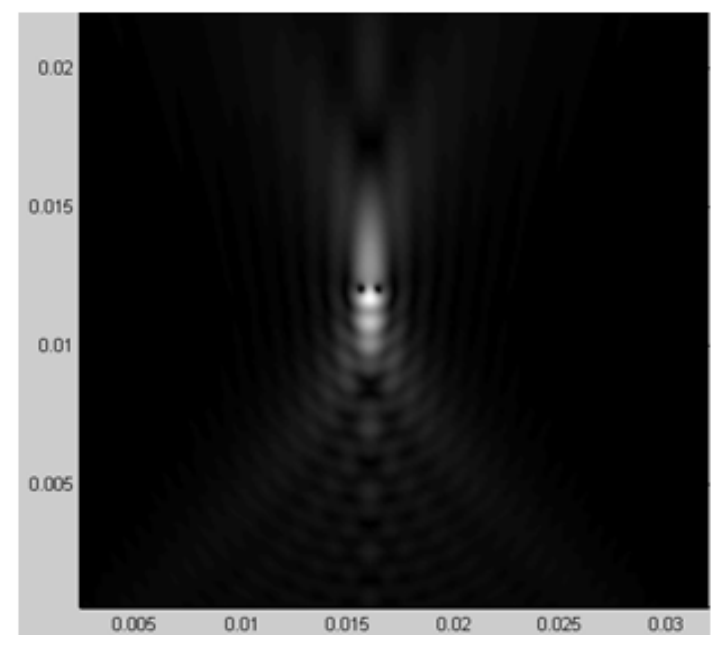

(a)

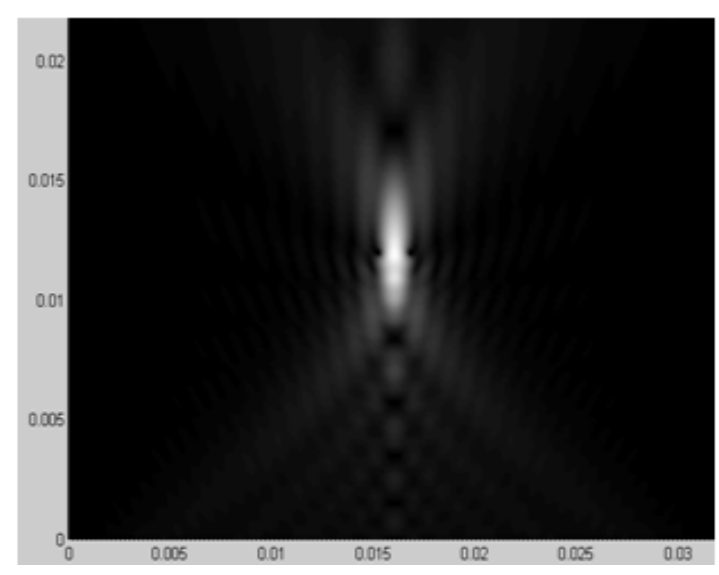

(c)

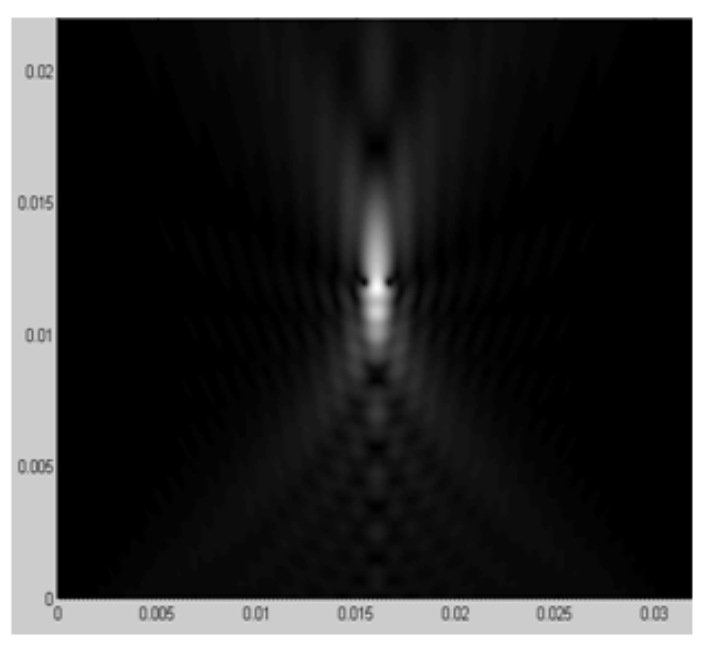

(b)

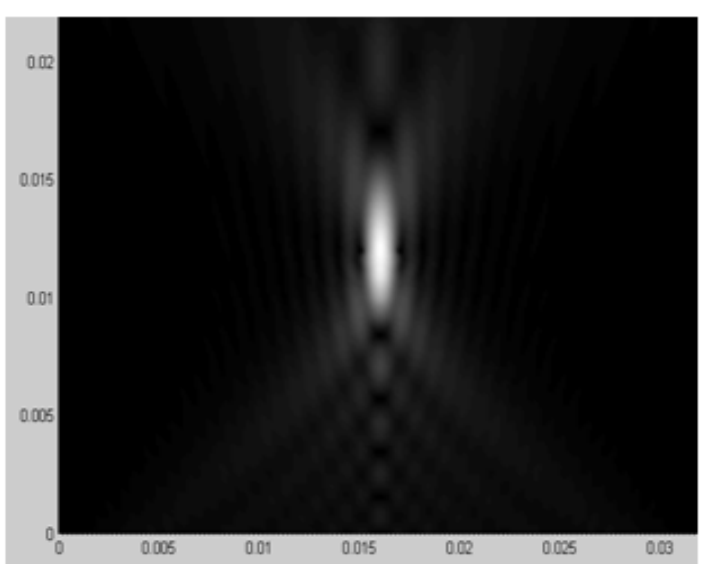

(d)

Figure 5.5 Computed pressure fields in front of the acoustic lens in the presence of two small cavities of radius = $0.1 \mathrm{~mm}$ as the distance between the two cavities is varied from $0.8 \mathrm{~mm}$ to $2 \mathrm{~mm}$ : (a) $0.8 \mathrm{~mm}$, (b) $1.2 \mathrm{~mm}$, (c) 1.6 $\mathrm{mm}$, and (d) $2.0 \mathrm{~mm}$. Numbers along the horizontal and vertical axes are given in meters and denote positions of the field points in a Cartesian coordinate system whose origin coincides with the bottom left corner of the figure. Scales are slightly different in the $x$ - and $y$-directions (see [85]). 


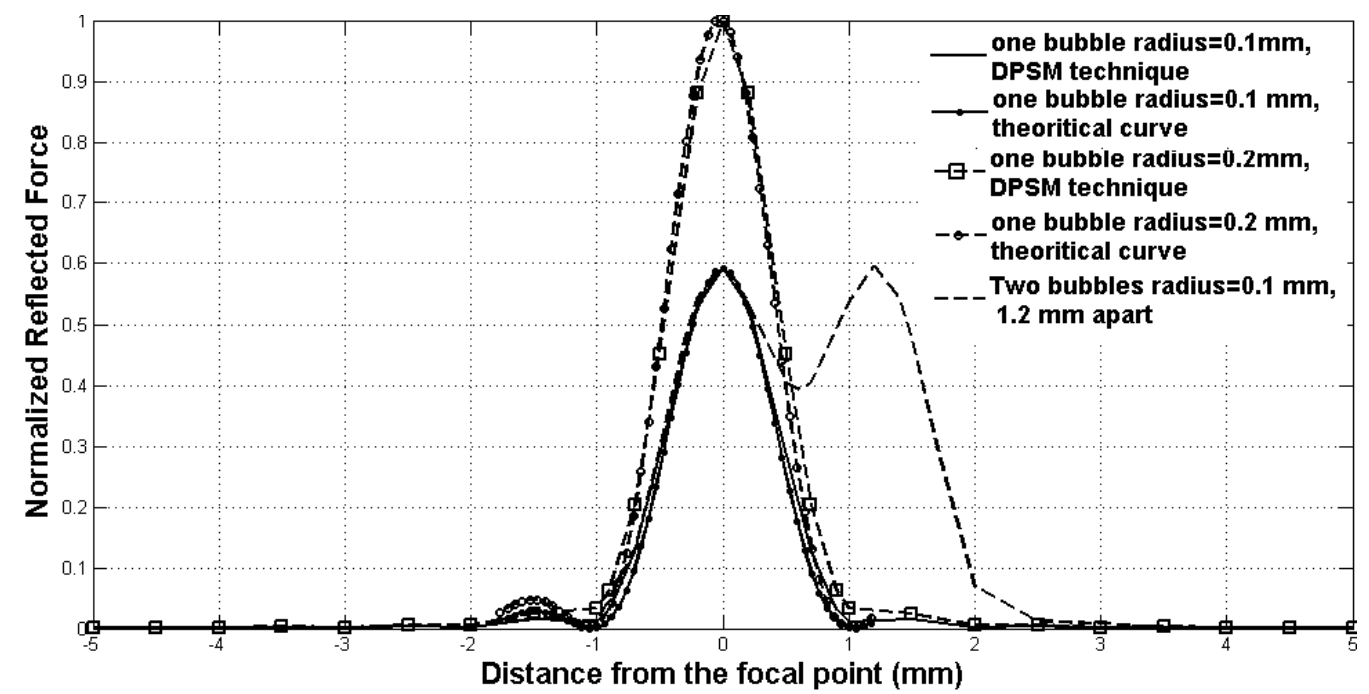

Figure 5.6 Normalized force sensed by the acoustic lens when the cavities move horizontally on the focal plane. The signal frequency = $1 \mathrm{MHz}$. Distance denotes the horizontal distance from the focal point to the center of the cavity (for the single-cavity case) or the center of the right cavity (for the two-cavity case). Distance between the cavity centers is $1.2 \mathrm{~mm}$ (see [85]) . 


\title{
APPENDIX A: DPSM3D FORTRAN SOURCE CODE FOR
}

\section{ULTRASONIC PROBLEMS}

\author{
Main Body of the code: \\ program DPSM_3d_ultrasonics \\ use Variables \\ use geometrylibrary \\ use meshing \\ use greensfunctions \\ use Excitation \\ implicit none \\ external ME42AD, ME 42BD, ME42CD, ME 42 ID, ME 42JD, ME 42PD \\ ! The folllowing variables are specific to Cavity on solid problem \\ real (8) : : sphx, sphx2, eas, ea, ds, eh, Rcurvature, sw

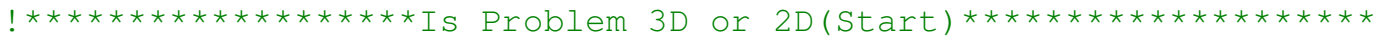 \\ Is 3Dproblem $=$. True.

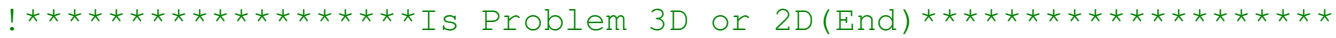

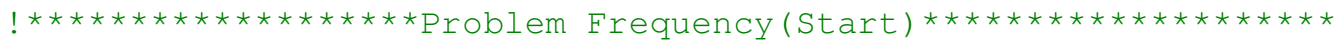 \\ Problemomega $=2 * \mathrm{pi} * 1 *(10 * * 6)$

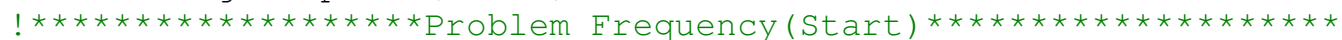

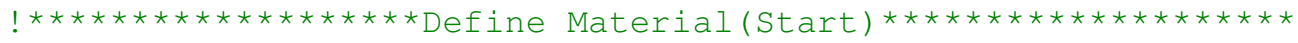 \\ Objects $\cdot$ nummediums $=7$ \\ allocate (Objects. Mediumlist (Objects.nummediums)) \\ objects.Mediumlist ( 1 ). mediumID=1 ! Water \\ Objects.Mediumlist(1). mediumtype=1 ! Fluid=1 \\ Objects. Mediumlist (1) . phoe $=1000$ \\ Objects. Mediumlist ( 1 ) . Cs=0 \\ Objects.Mediumlist ( 1 ) . $\mathrm{Cp}=1480.0$ \\ Objects.Mediumlist (2).mediumID=2 ! Aluminum \\ Objects.Mediumlist (2). mediumtype=2 !Solid=2 \\ Objects. Mediumlist (2). landa=1.6489* (10**10) \\ Objects. Mediumlist (2). miu=2.5883* (10**10) \\ Objects.Mediumlist (2) . poisson=0.349 \\ Objects. Mediumlist ( 2 ) phoe $=2200.0$ \\ Objects. Mediumlist (2). Cs $=3.43 * 1000$ \\ Objects. Mediumlist (2) . $\mathrm{Cp}=5.57 * 1000$ \\ Objects.Mediumlist (4).mediumID=4 !Glycerine \\ Objects.Mediumlist (4). mediumtype=1 ! Fluid=1 \\ objects. Mediumlist (4). phoe=1000 !1260 \\ Objects. Mediumlist ( 4$) \cdot \mathrm{Cs}=0$ \\ Objects.Mediumlist (4). $\mathrm{Cp}=1480$ !1920.0
}




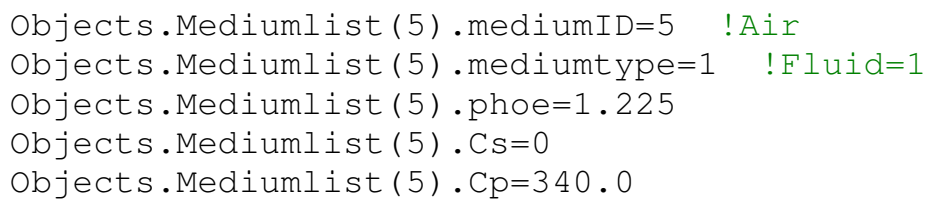




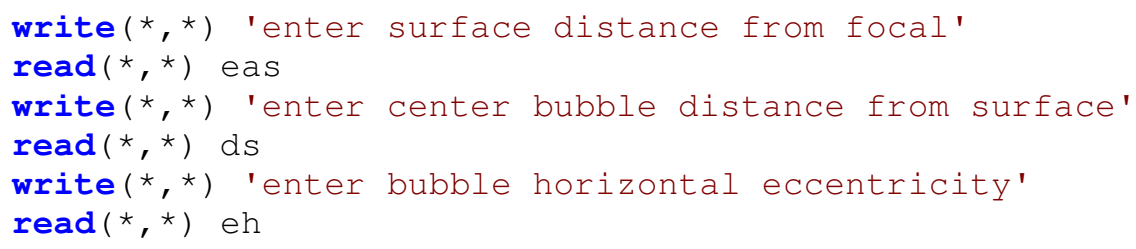

Objects.hemispherelist (2). surfaceID=4

Objects.hemispherelist (2) . origin=(/dble (eh), dble (0.0), dble (0.02+ea)/)

Objects.hemispherelist (2).radius=abs (Rcurvature)

objects.hemispherelist (2). teta $=2.0 *(180 / \mathrm{pi}) * a \sin (\mathrm{sw} * 0.5 / \mathrm{abs}$ (Rcurvature) )

if (Rcurvature>0) then

Objects.hemispherelist (2) . normalvect $=(/ 0.0,0.0,1.0 /)$

objects.hemispherelist ( 2 ) . outsidemedium=2

objects.hemispherelist (2). insidemedium=1

else 
Objects.hemispherelist (2) . normalvect $=(/ 0.0,0.0,-1.0 /)$

Objects.hemispherelist (2) . outsidemedium=1

objects.hemispherelist (2) . insidemedium=2

endif

Objects . numpolygons $=0$

Objects $\cdot$ numcircles $=0$

objects. numhemispheres $=2$

objects $\cdot$ numspheres $=2$

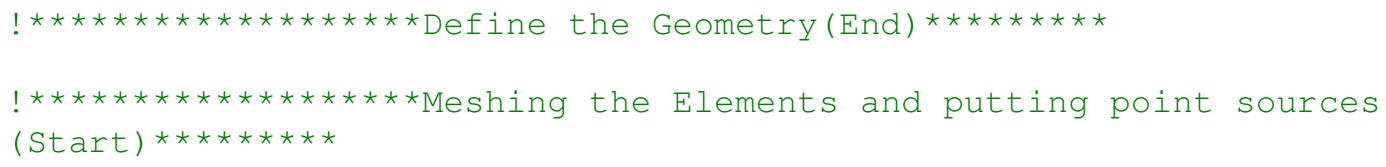


if (CPmedium1.mediumtype==1. and. CPmedium2.mediumtype==1) then !FluidFluid Interface

Objects.DOFlist (counter). Interfacecase $=1$

Objects. DOFlist (counter) $. \mathrm{CPDOF}=$ lastCPDOF+1

Objects. DOFlist (counter) . numCPDOF $=2$

last $\mathrm{CPDOF}=$ last $\mathrm{CPDOF}+2$

Objects.DOFlist (counter). $\operatorname{PSDOF}(1)=$ lastPSDOF +1

Objects.DOFlist (counter) $\cdot \operatorname{PSDOF}(2)=$ lastPSDOF +2

Objects. DOFlist (counter) . numPSDOF $(1)=1$

objects.DOFlist (counter) $\operatorname{numPSDOF}(2)=1$

lastP $\mathrm{SDOF}=1$ ast $\mathrm{PSDOF}+2$

else if

( ( CPmedium1. mediumtype==1. and. CPmedium2. mediumtype==2). or. ( CPmedium1.me diumtype $==2$. and. CPmedium2.mediumtype==1)) then ! Fluid-Solid Interface

objects.DOFlist (counter). Interfacecase $=2$

Objects.DOFlist (counter) $. \mathrm{CPDOF}=$ last CPDOF+1

Objects. DOFlist (counter) . numCPDOF $=4$

last $\mathrm{CPDOF}=1$ ast $\mathrm{CPDOF}+4$

if (CPmediuml.mediumtype==1) then

Objects.DOFlist (counter). PSDOF ( 1 ) =lastPSDOF +1

Objects.DOFlist (counter) $\cdot \operatorname{PSDOF}(2)=1$ astPSDOF +2

objects. DOFlist (counter) $\operatorname{numPSDOF}(1)=1$

objects. DOFlist (counter) $\operatorname{numPSDOF}(2)=3$

\section{else}

Objects. DOFlist (counter). PSDOF ( 1 ) =lastPSDOF +1

Objects.DOFlist (counter). PSDOF ( 2 ) =lastPSDOF +4

objects. DOFlist (counter) . numPSDOF $(1)=3$

Objects. DOFlist (counter) $\operatorname{numPSDOF}(2)=1$

\section{end if}

lastP $\mathrm{SDOF}=$ lastP $\mathrm{SDOF}+4$

else if (CPmedium1.mediumtype==2. and.CPmedium2. mediumtype==2) then !Solid-Solid Interface

Objects.DOFlist (counter). Interfacecase $=3$

Objects.DOFlist (counter) $. \mathrm{CPDOF}=$ last CPDOF+1

objects.DOFlist ( counter) $\cdot$ numCPDOF $=6$

last $\mathrm{CPDOF}=$ last $\mathrm{CPDOF}+6$

Objects.DOFlist (counter). PSDOF ( 1 ) =lastPSDOF+1

objects.DOFlist (counter) $\cdot \operatorname{PSDOF}(2)=$ lastPSDOF +4

objects.DOFlist (counter). numPSDOF $(1)=3$

Objects.DOFlist (counter) . numPSDOF (2) $=3$

lastP $\mathrm{SDOF}=$ lastP $\mathrm{SDOF}+6$

\section{else if}

( ( CPmedium1. mediumtype==1. and. CPmedium2. mediumtype==0). or. ( CPmedium1.me diumtype $=0$. and. CPmedium2. mediumtype=1)) then ! Fluid-Transducer

Interface

Objects.DOFlist (counter). Interfacecase=4 !Fluid-Transducer

interface $=4$

Objects. DOFlist ( counter) $\cdot \mathrm{CPDOF}=$ last $\mathrm{CPDOF}+1$

objects.DOFlist ( counter) . numCPDOF $=1$

last $\mathrm{CPDOF}=$ last $\mathrm{CPDOF}+1$

if (CPmedium1.mediumtype==1) then

Objects.DOFlist (counter). PSDOF ( 1 ) =lastPSDOF +1

Objects.DOFlist (counter) $\cdot \operatorname{PSDOF}(2)=0$

objects. DOFlist (counter) $\operatorname{numPSDOF}(1)=1$ 


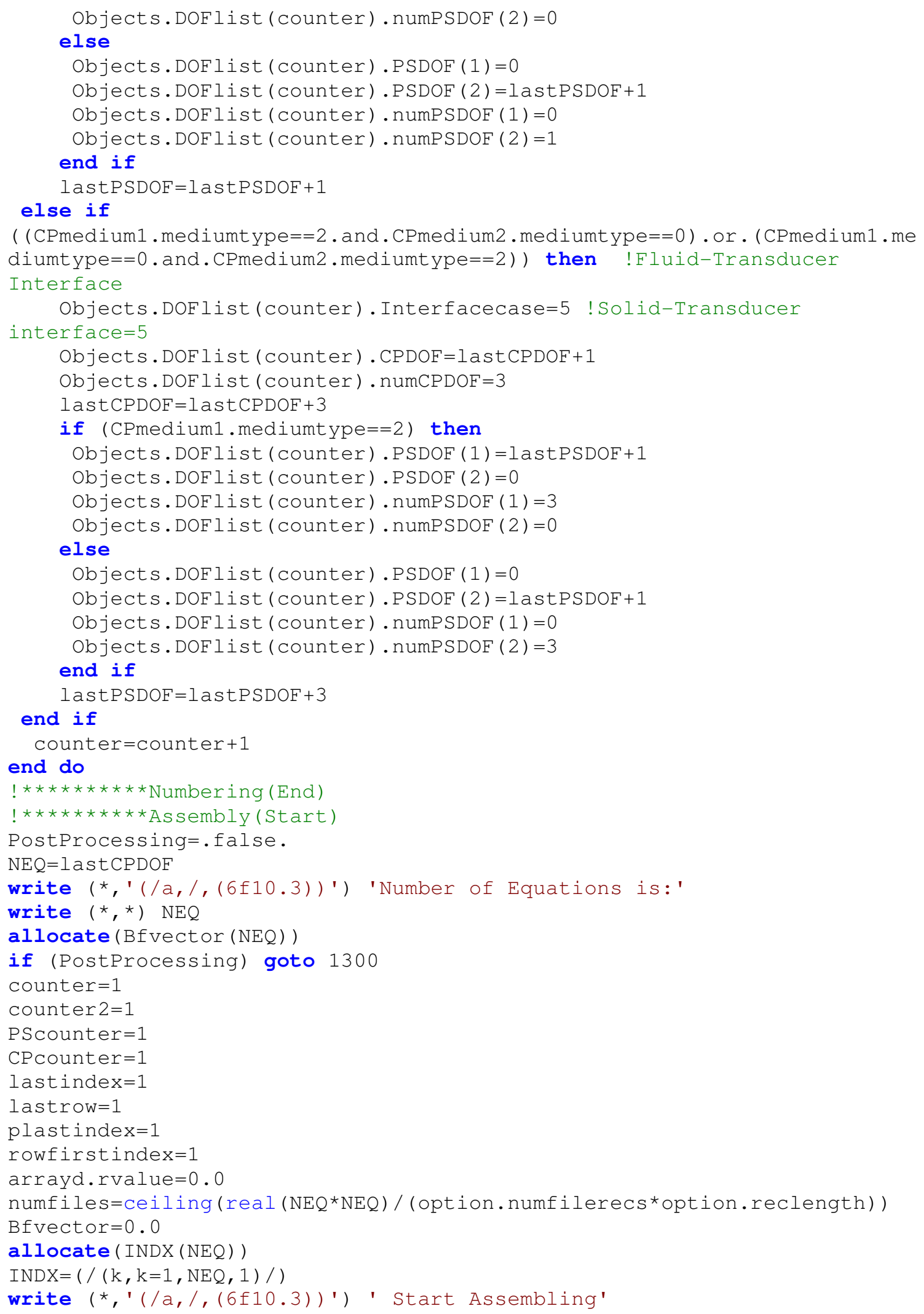




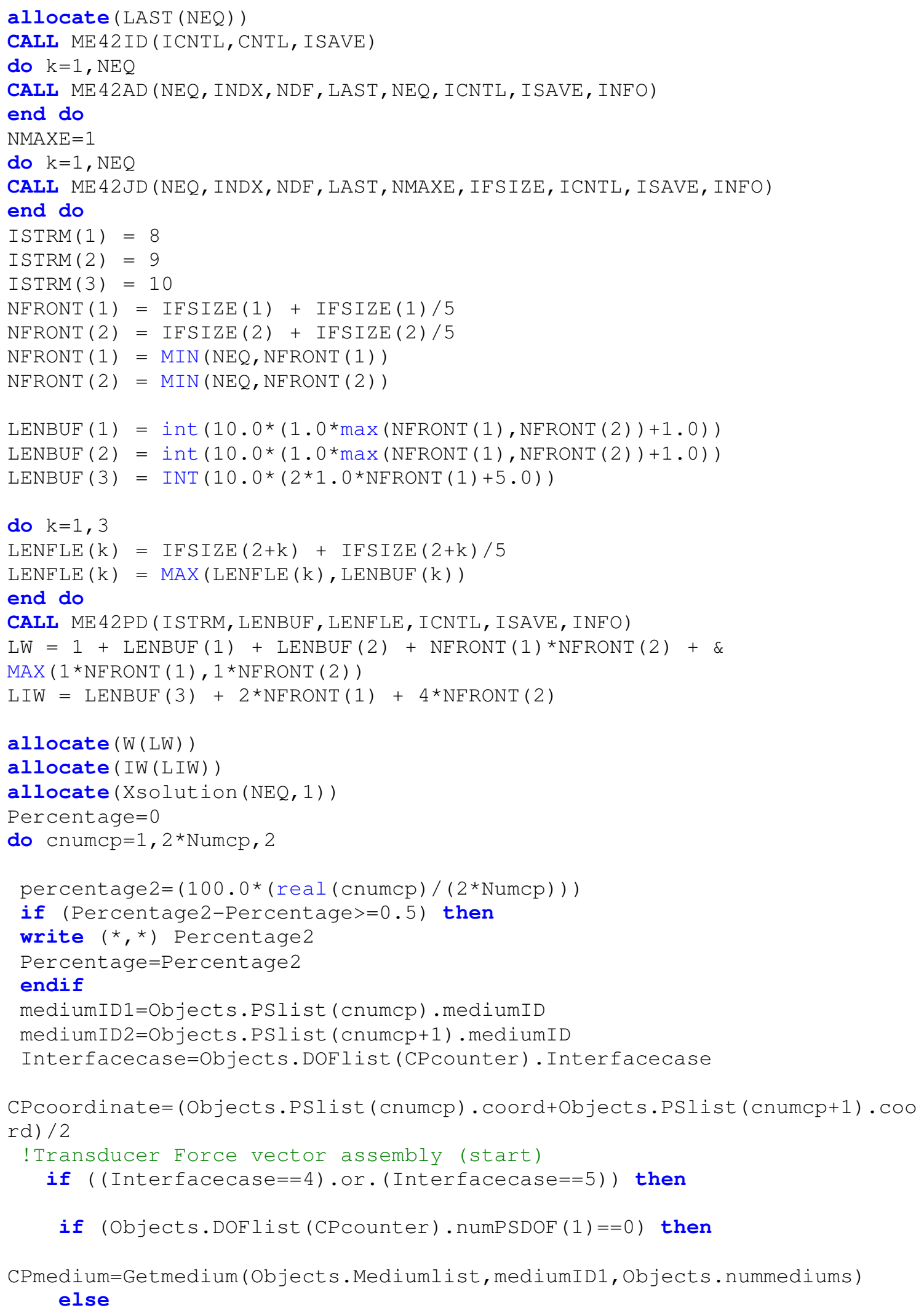




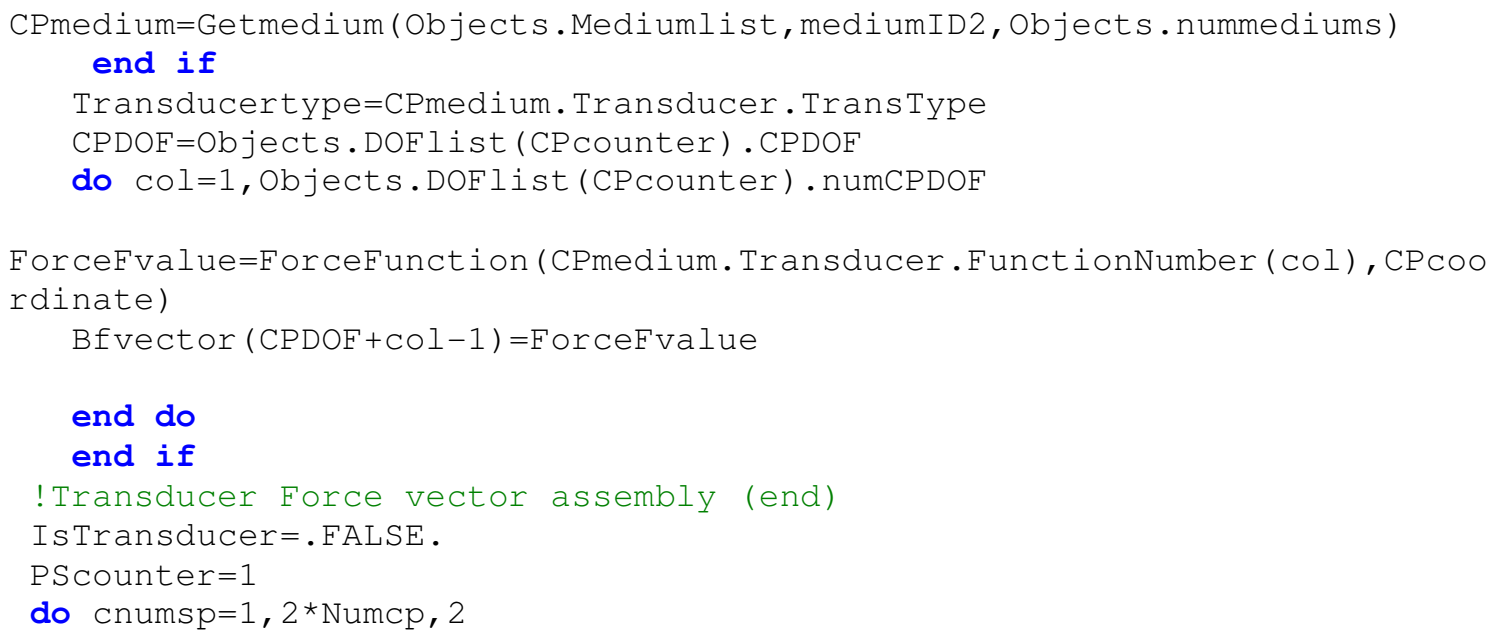

ForceFvalue=ForceFunction (CPmedium. Transducer. FunctionNumber ( col), CPcoo rdinate)

Bfvector $(\mathrm{CPDOF}+\mathrm{COl}-1)=$ ForceFvalue

\section{end do} end if

! Transducer Force vector assembly (end)

IsTransducer $=$. FALSE.

PScounter $=1$

do cnumsp $=1,2 *$ Numcp, 2

SPcoordinate $=($ objects.PSlist (cnumsp) . coord+Objects.PSlist $($ cnumsp+1) . coo rd) $/ 2$

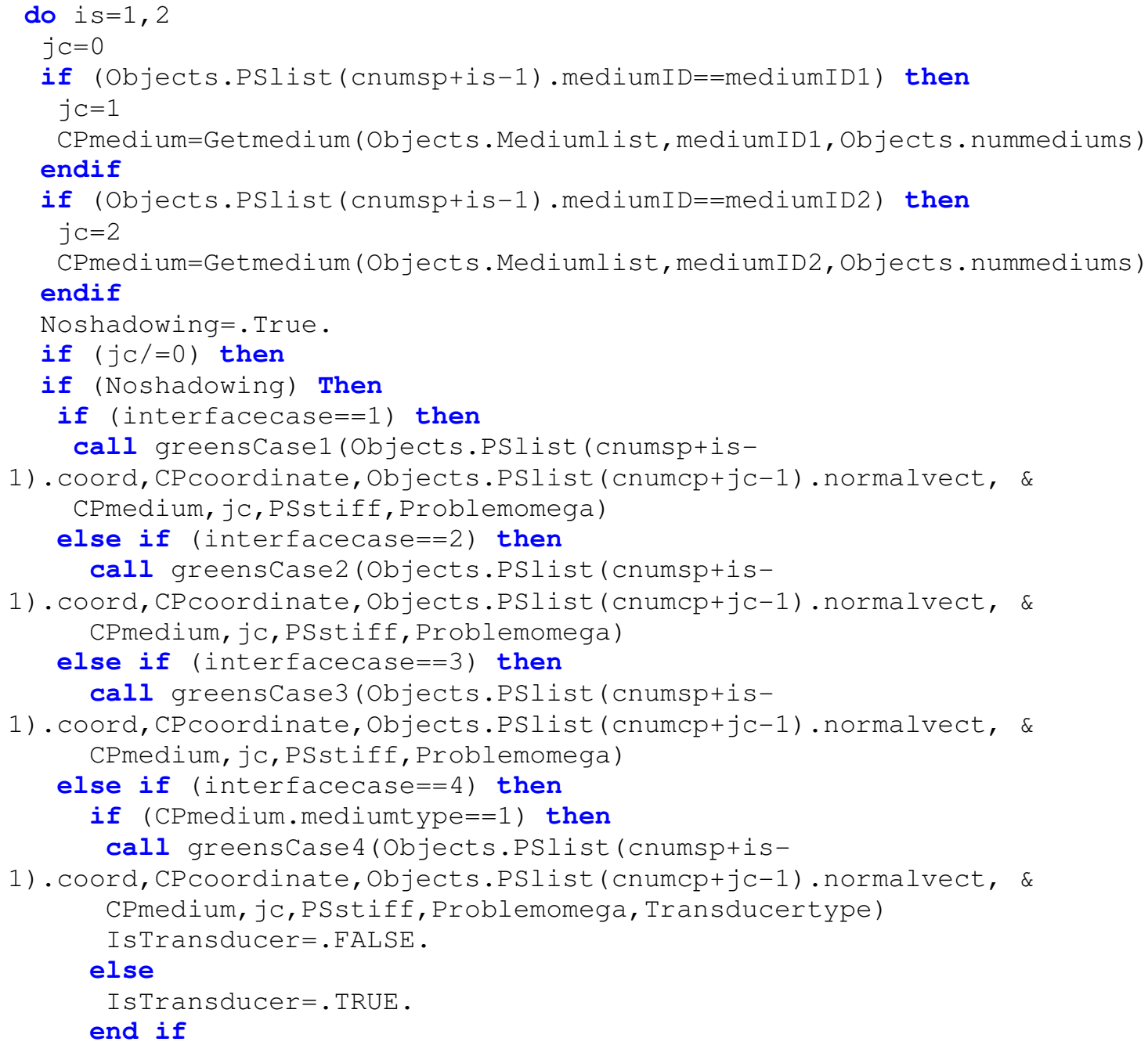


else if (interfacecase $==5$ ) then

if (CPmedium.mediumtype==2) then

call greenscase5(Objects.PSlist (cnumsp+is-

1). coord, CPcoordinate, Objects.PSlist (cnumcp+jc-1).normalvect, \&

CPmedium, jc, PSstiff, Problemomega, Transducertype)

IsTransducer $=$.FALSE.

else

IsTransducer $=$. TRUE.

end if

end if

if (.not.IsTransducer) then

PSDOF $=$ Objects. DOFlist (PScounter). PSDOF (is)

numsubrows=size (PSstiff, 1$)$

if (.not.allocated (subrowBuffer)) then

allocate (subrowBuffer (numsubrows, NEQ))

subrowBuffer $=0.0$

endif

subrowBuffer (:,PSDOF :PSDOF+size (PSstiff,2)-1)=PSstiff

end if

endif !shadowing check if

end if !end for "if $j /="$

end do ! for $i=1,2$

PScounter $=$ PScounter +1

end do

do $\mathrm{k}=1$, numsubrows

$\operatorname{RHS}(1,1)=$ Bfvector $($ lastrow $+\mathrm{k}-1)$

CALL ME42BD (NEQ, INDX, NDF, LAST, NMAXE, subrowBuffer $(k: k,:), 1, \operatorname{RHS}, 1, \mathrm{NEQ}, \&$

Xsolution, NFRONT, LENBUF, LW, W, LIW, IW, ICNTL, CNTL, ISAVE, INFO, RINFO)

end do

deallocate (subrowBuffer)

lastrow $=$ lastrow + numsubrows

CPcounter $=$ CPcounter +1

end do

deallocate $(W)$

deallocate (IW)

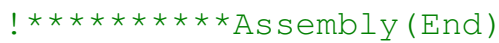

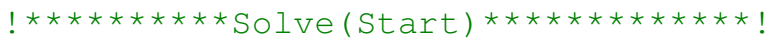

open (1000, FILE="ForceVector. DPSM", status='replace' )

write $(1000$, *) Bfvector

close (1000)

write (*,' (/a,/,(6f10.3))') ' Start Solving: '

open (1000, FILE="Xsolution. DPSM", status='replace' )

write $(1000$, *) Xsolution

close ( 1000 )

1300 if (PostProcessing) then

open (1000, FILE="Xsolution.DPSM", status='Old')

allocate (Xsolution (NEQ, 1))

$\operatorname{read}(1000, *)$ Xsolution

close (1000)

endif 


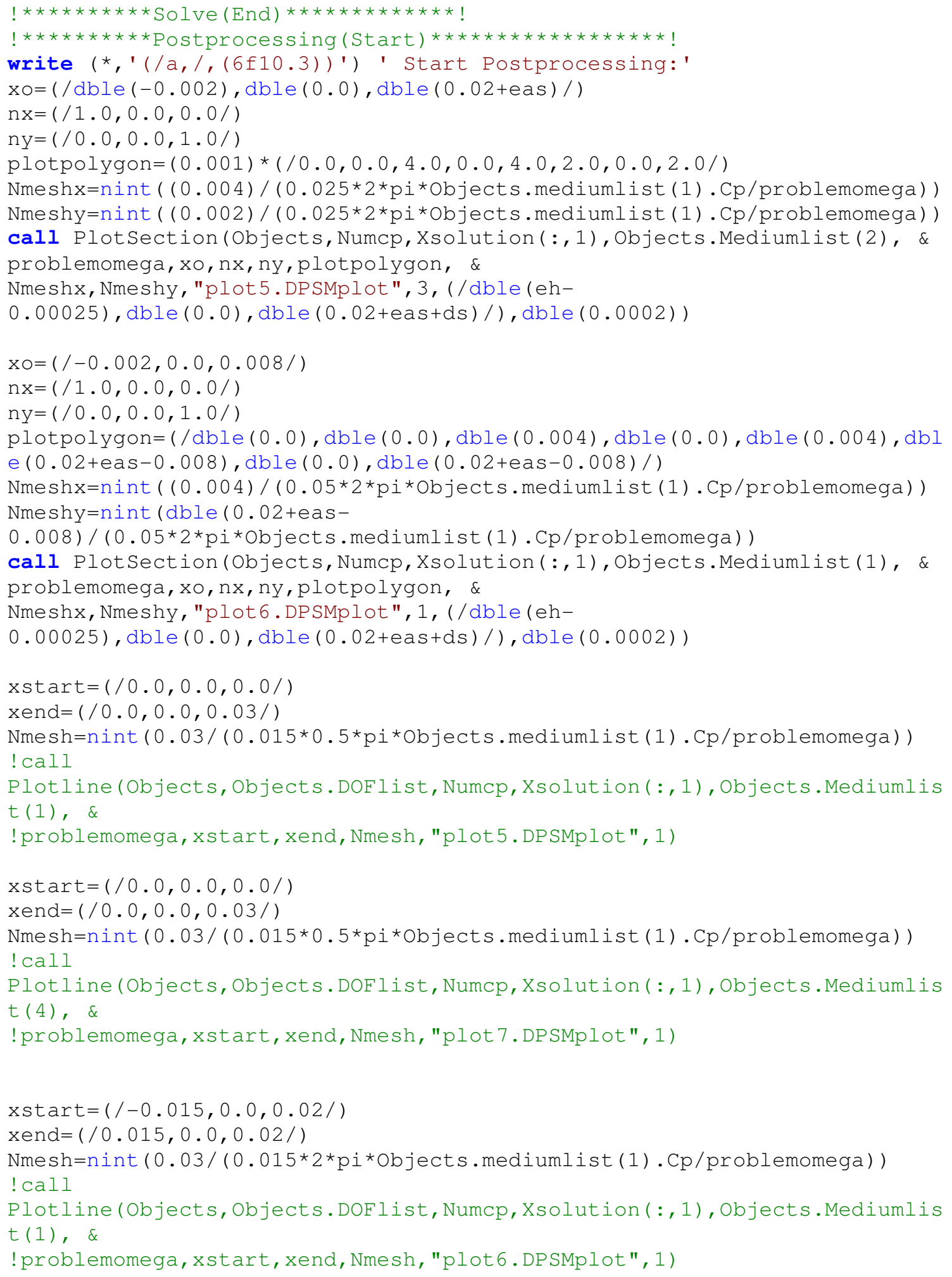


Meshsize=Meshsize*0.5

Energy=HemisphereEnergy (Objects, Objects.hemispherelist (1), Meshsize, Obje cts.DOFlist, \&

Numcp, Xsolution ( :, 1), Objects.Mediumlist (1), problemomega, 1 )

write (*,'(/a,/,(6f10.3))') ' Real Energy='

write $(*, *)$ real (Energy)*(Meshsize**2)

write $(*, '(/ a, /,(6 f 10.3))$ ') ' imaginary Energy='

write $(*, *)$ imag (Energy)* (Meshsize**2)

write (*,' (/a,/,(6f10.3))') ' Absoloute Energy='

write $(*, *)$ abs (Energy)* (Meshsize**2)

write $(*, '(/ \mathrm{a}, /,(6 f 10.3))$ ') ' Completed'

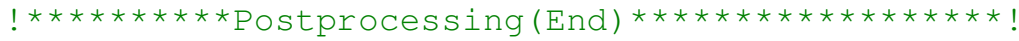

read*

end program 


\section{"Greensfunctions" Module:}

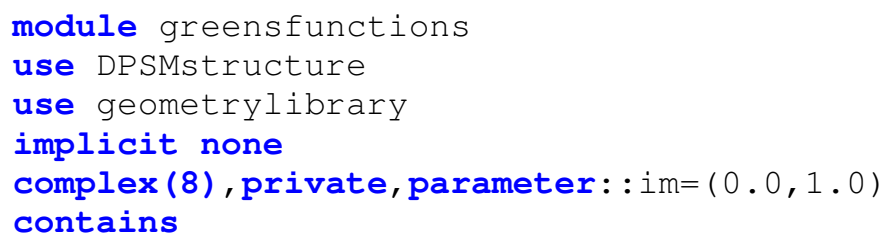




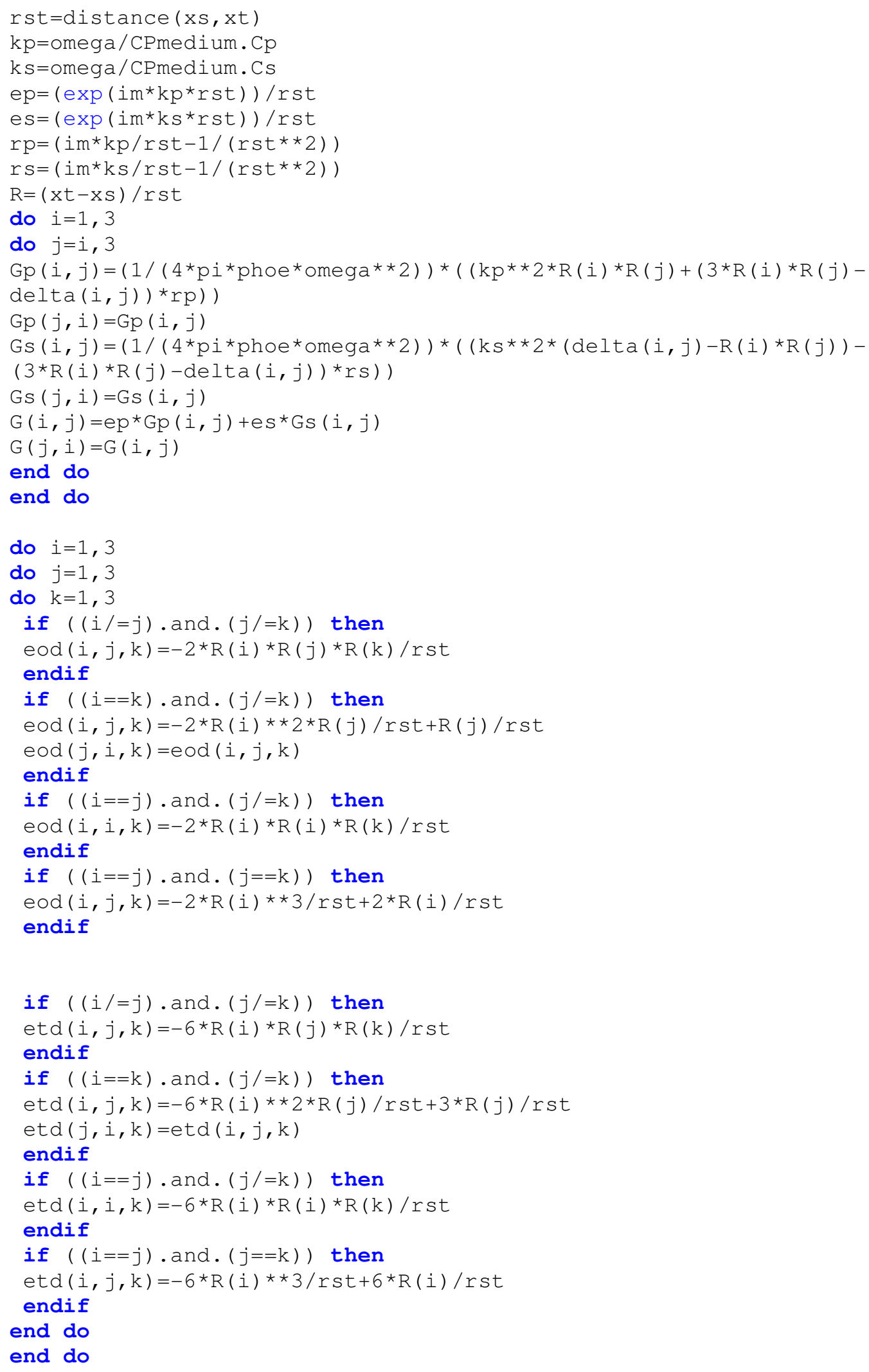




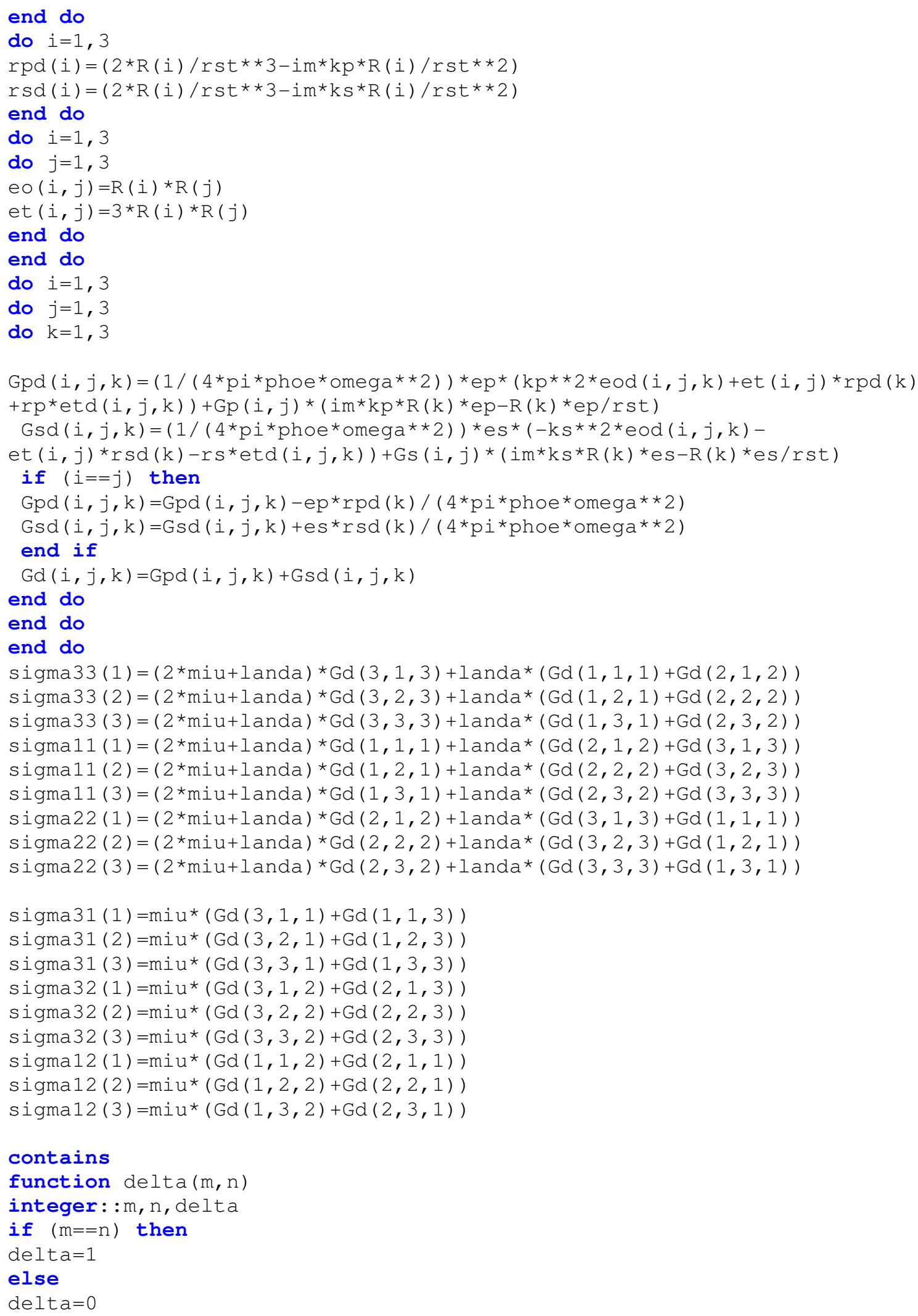




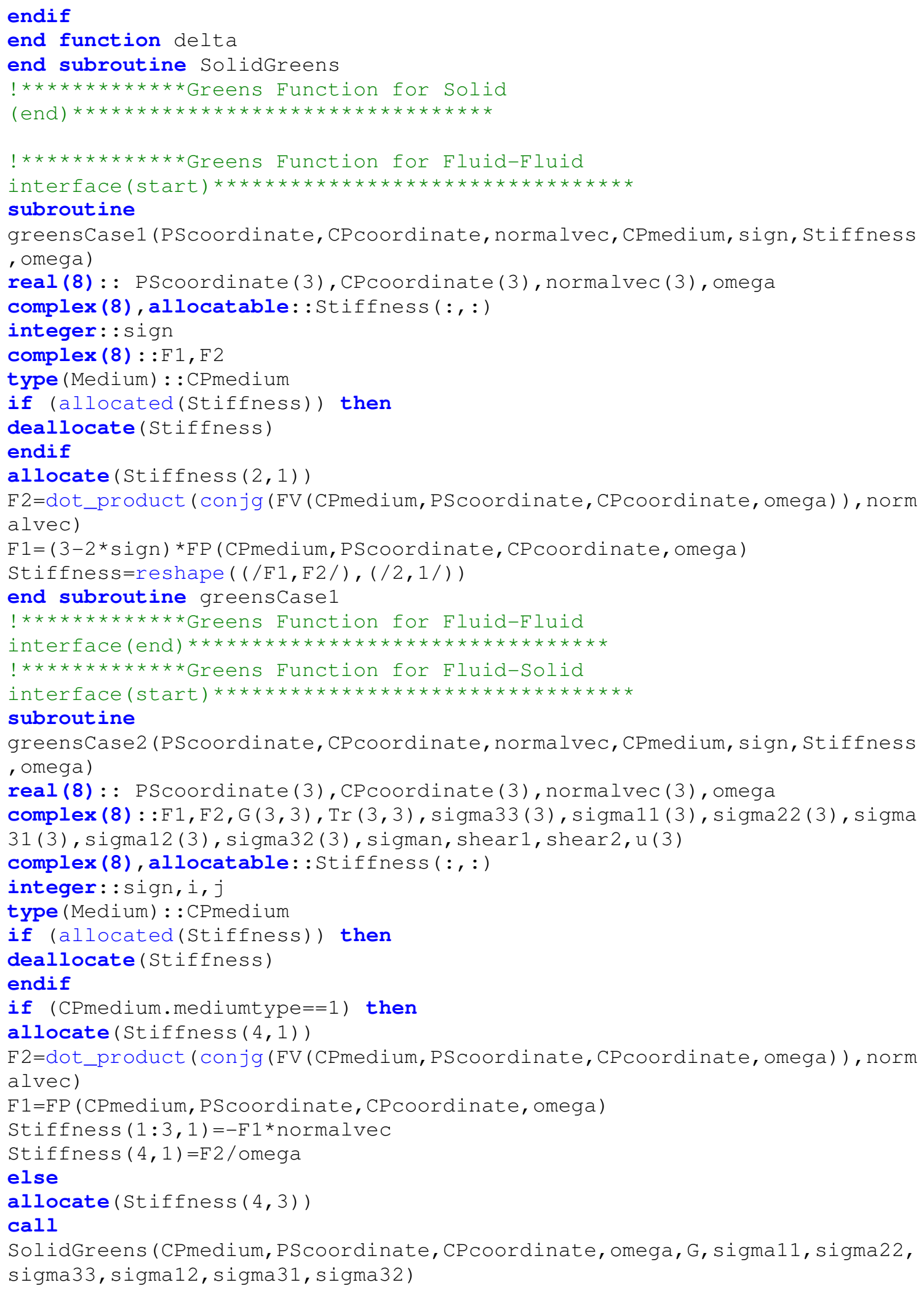




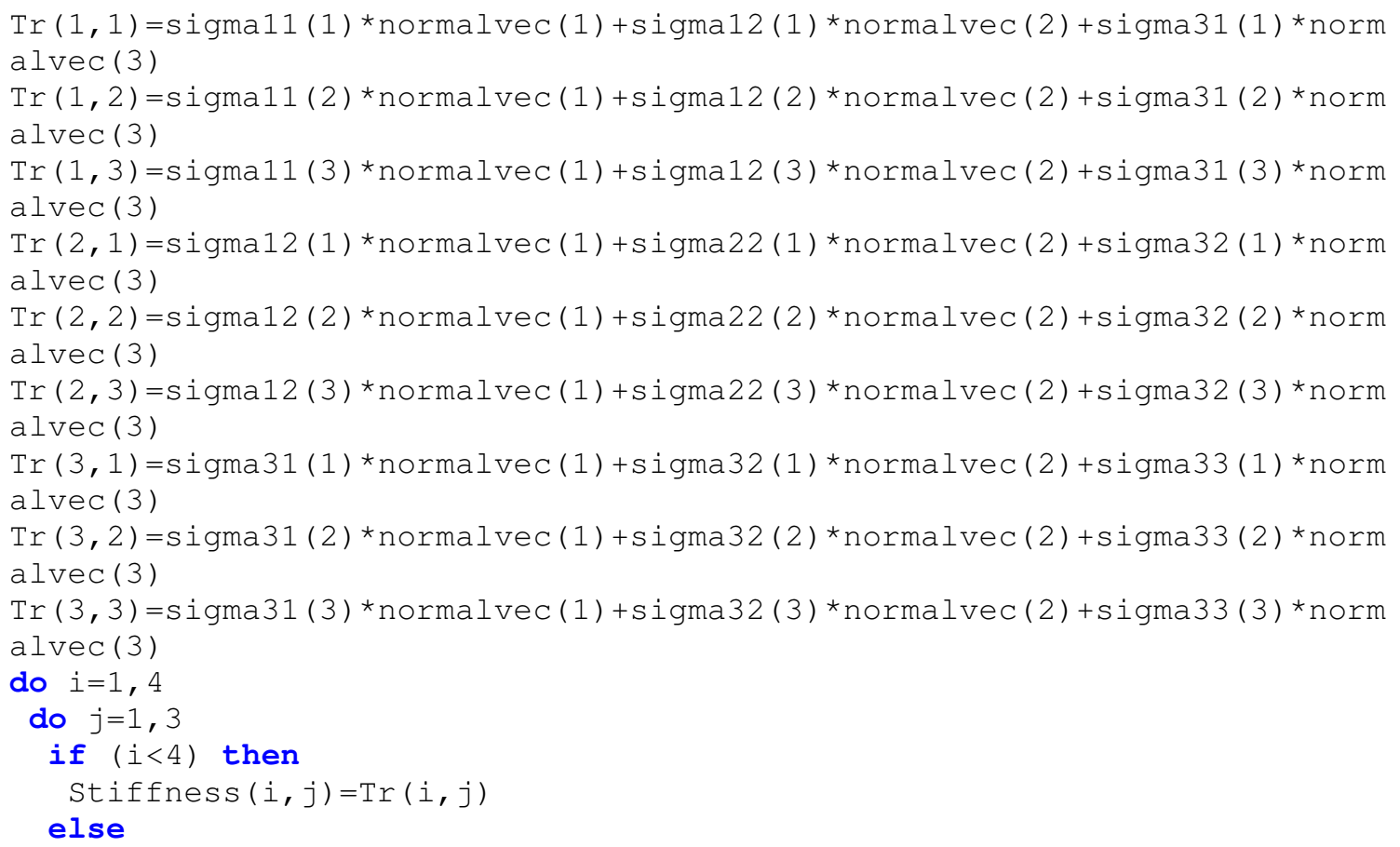

Stiffness $(i, j)=G(1, j)$ *normalvec $(1)+G(2, j)$ *normalvec $(2)+G(3, j)$ *normalvec (3) 


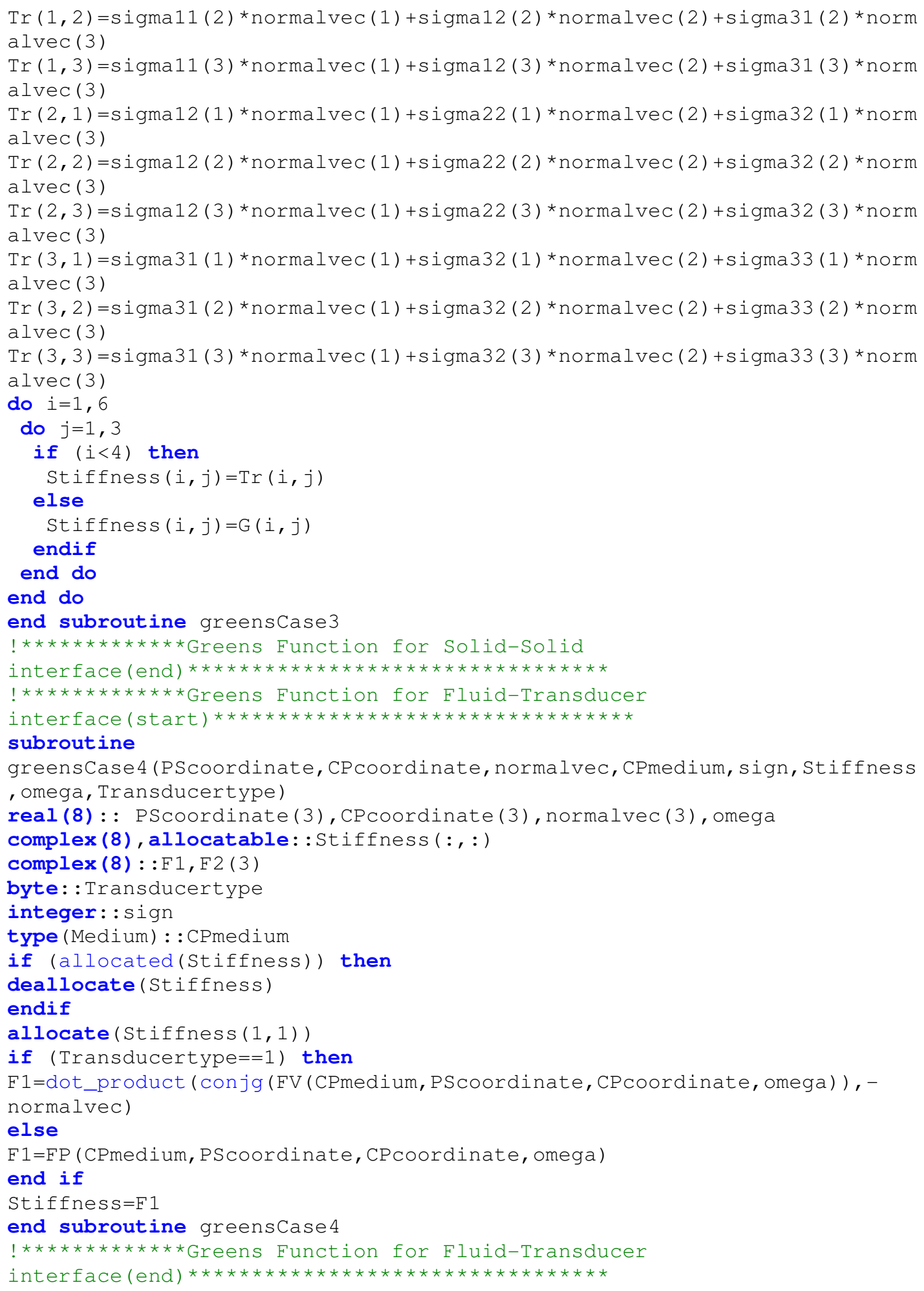




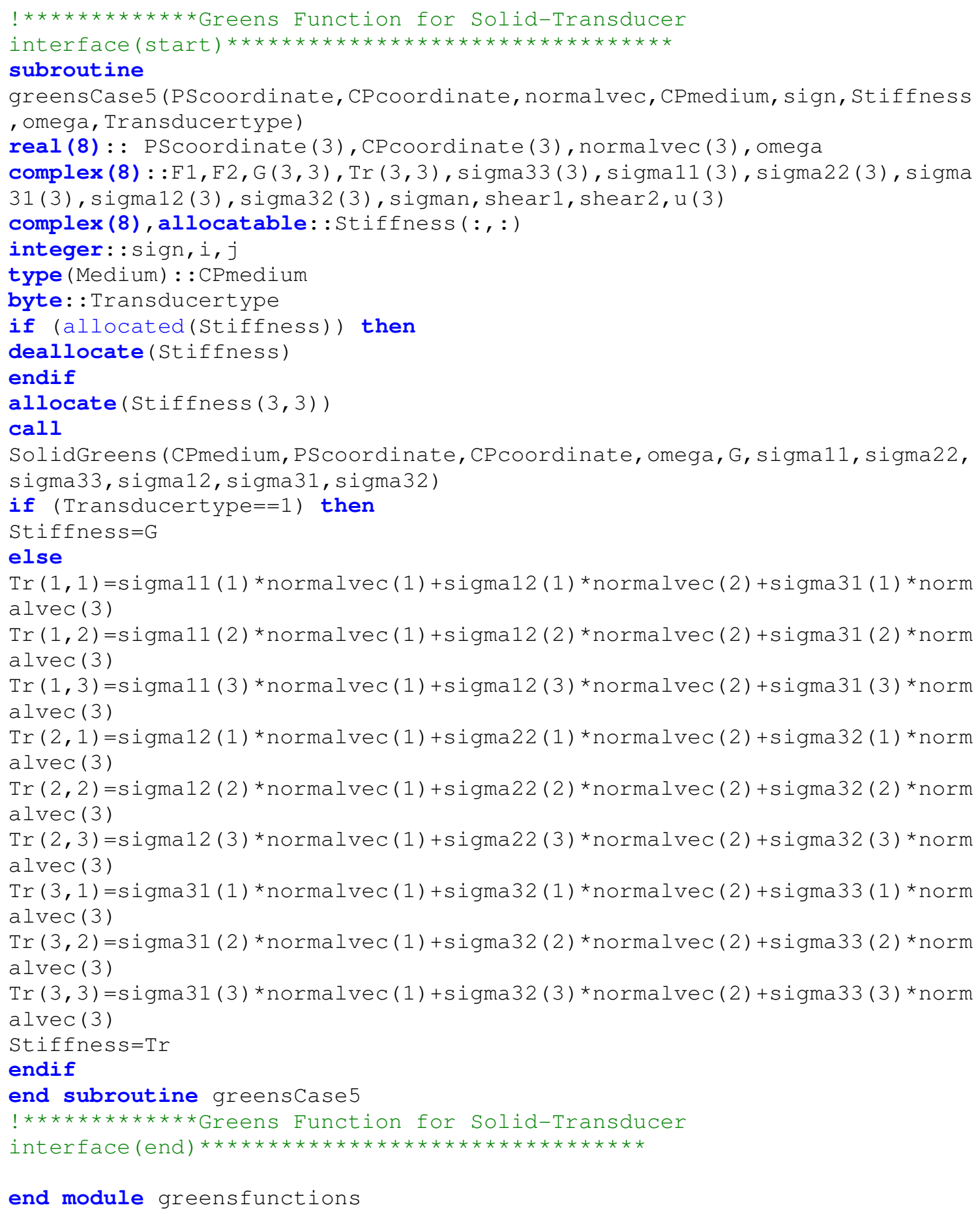




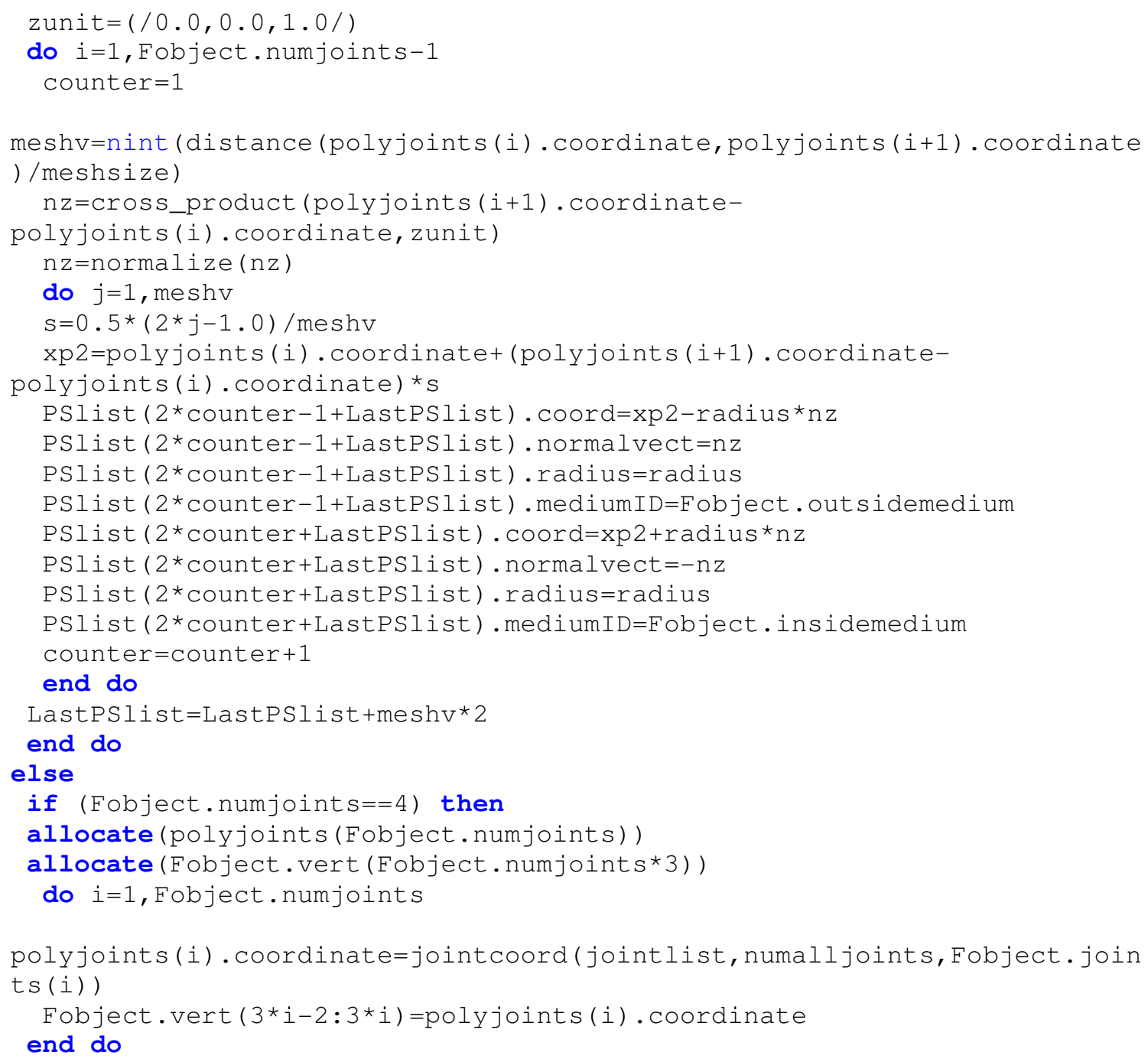




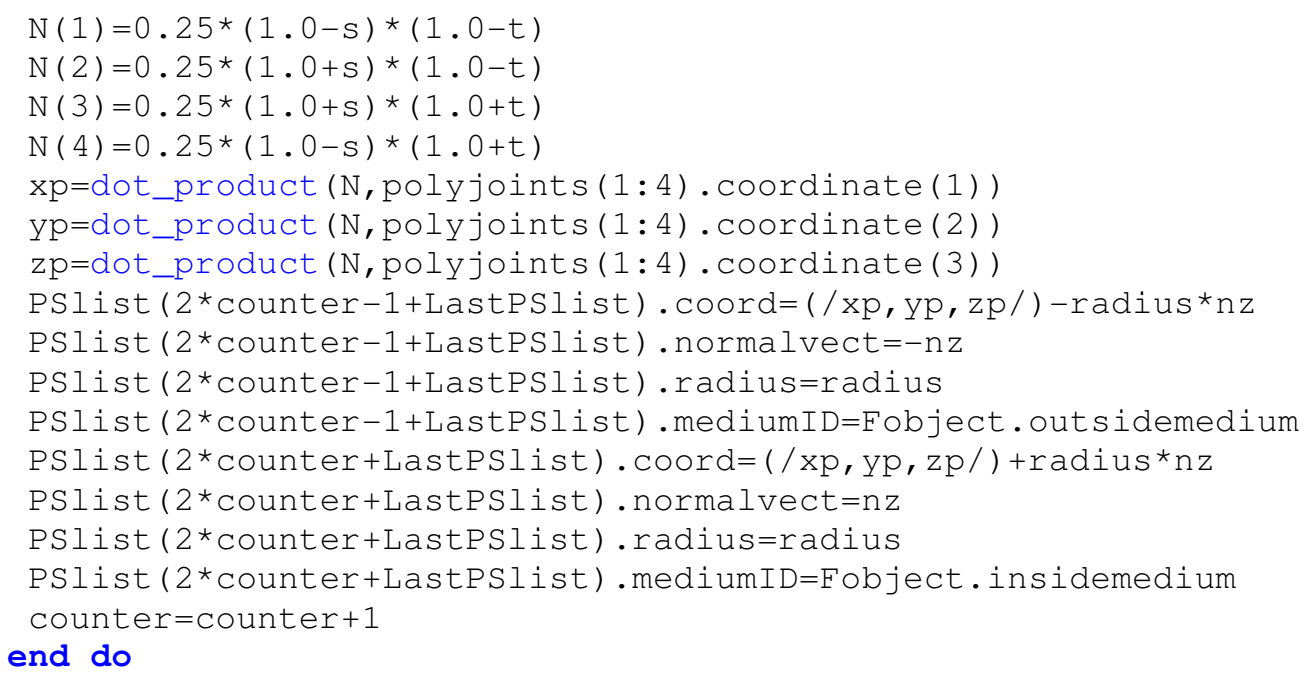

end do

LastPSlist $=$ LastPSlist $+($ meshh $)$ * $($ meshv $) * 2$

else

end if

endif

if (allocated(polyjoints)) deallocate(polyjoints)

end subroutine PolygonMesh

! $* * * * * * * * * * * * *$ Function PolygonMesh(it meshes a n-sided polygon and puts

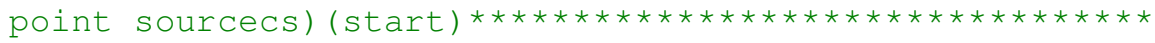

! $* \star \star \star * \star * \star * \star * \star *$ Function PlotMeshgrid(it meshes a 4-sided polygon and

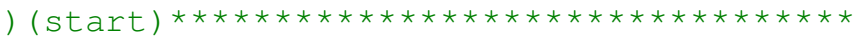

\section{subroutine}

PlotSection (Objects, Numcp, strength, Plotmedium, omega, xo, nx, ny, polygon, Nm eshx, Nmeshy, file_name, outputcase, bcenter, bradius)

real (8): :s, t, N (4), nz (3), nx (3), ny (3), sp,tp, Tpcoord (3), Spcoord (3), CPcoord (3), xo (3), polygon (8), bcenter (3), bradius

real (8) : : test, d1, d2, d3, d4

character (len=*) : : file_name

complex (8) : : outputvalue, FV2 (3) , im2

type (Medium) : :Plotmedium

type (DPSMobjects) : : objects

real (8) : : omega, tolerance

complex (8): : strength (:)

integer: :

m, Nmeshx, Nmeshy, outputcase, meshs, mesht, i, j, k, cp, counter, cpcounter, Numcp , PSdof, numPSdof

logical: : notshadowed

complex (8):: G(3,3), sigma33 (3), sigma11(3), sigma22(3), sigma31(3), sigma12( 3), sigma32(3)

$\operatorname{im} 2=(0.0,1.0)$

tolerance $=1.0$

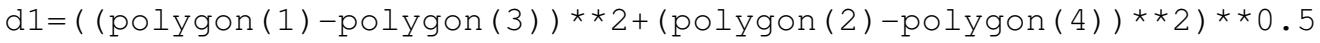

$\mathrm{d} 2=($ (polygon $(7)$-polygon ( 5$)) \star \star 2+($ polygon $(8)-$ polygon $(6)) \star \star 2) \star \star 0.5$

$\mathrm{d} 3=(($ polygon $(1)-$ polygon $(7)) * \star 2+($ polygon $(2)-\operatorname{polyg}$ yon $(8)) * \star 2) * \star 0.5$ 


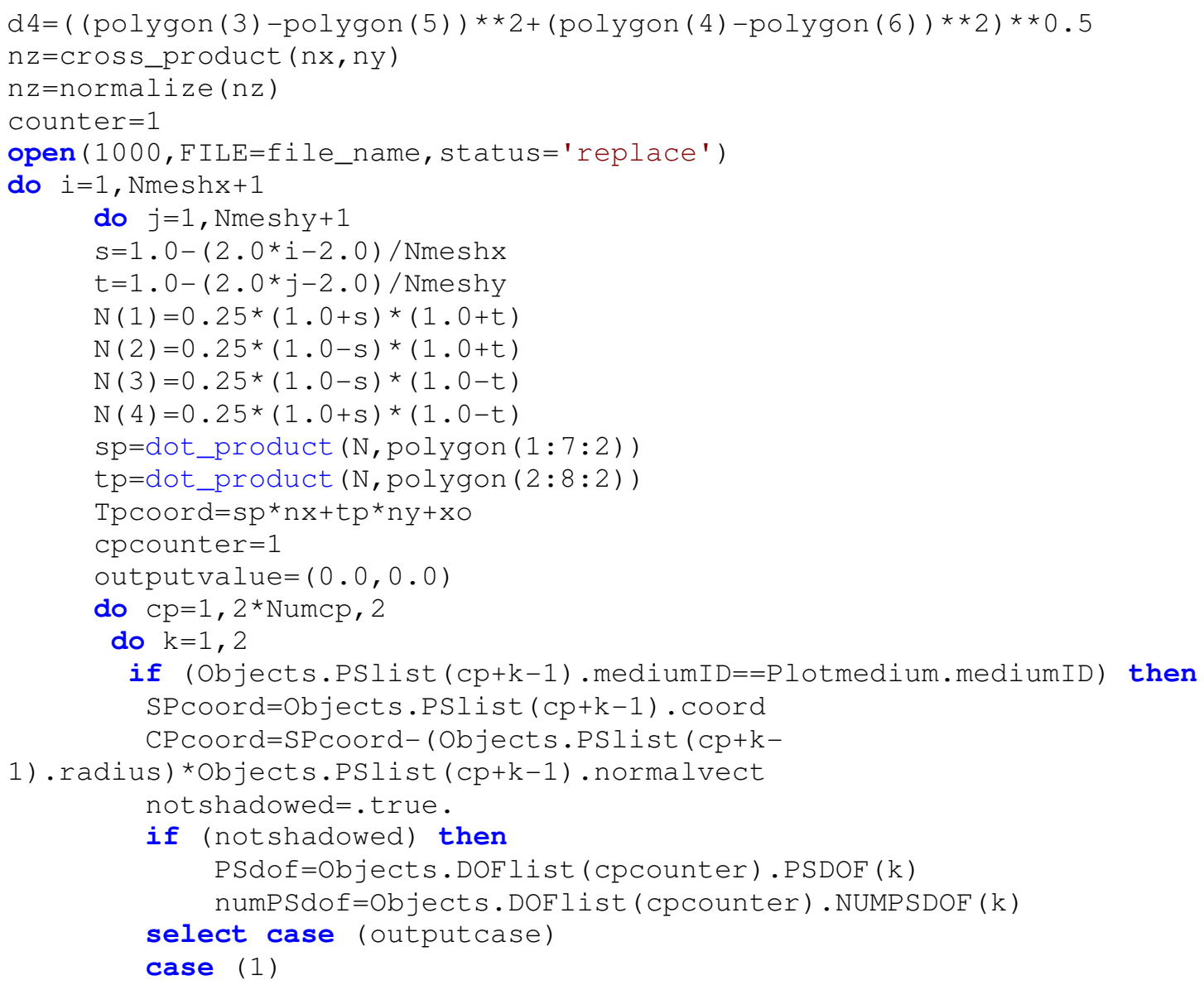




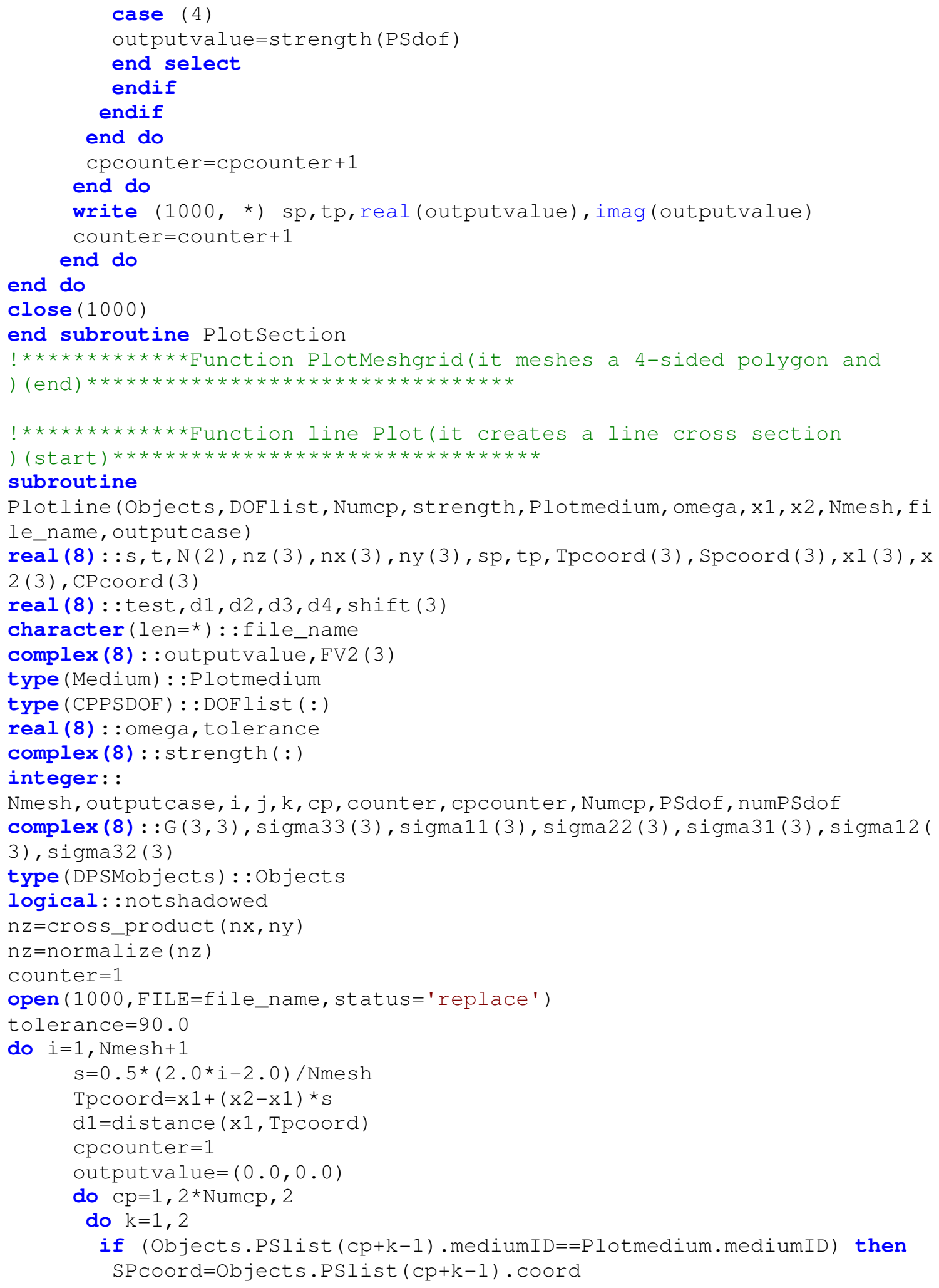


CPCoord=SPcoord $-($ Objects.PSlist $(\mathrm{cp}+\mathrm{k}-$

1 ).radius) *objects.PSlist $(\mathrm{cp}+\mathrm{k}-1)$. normalvect

not shadowed=.true.

! Check shadowing (End)

if (notshadowed) then

PSdof=objects. DOFlist ( cpcounter).PSDOF (k)

numPSdof=Objects. DOFlist (cpcounter). NUMPSDOF (k)

select case (outputcase)

case (1)

outputvalue=outputvalue+strength (PSdof) *FP (plotmedium, SPcoord, Tpcoord,o mega)

case (2)

FV2=FV (plotmedium, SPcoord, Tpcoord, omega)

outputvalue=outputvalue+strength (PSdof) *FV2 (3)

case (3)

call

SolidGreens (plotmedium, SPcoord, TPcoord, omega, G, sigma11, sigma22, sigma33, sigma12, sigma31, sigma32)

outputvalue=outputvalue+strength (PSdof) *sigma32(1) +strength (PSdof+1) * $i$ gma32 $(2)+\&$

strength $($ PSdof +2$) * \operatorname{sigma32}(3)$

case (4)

call

SolidGreens (plotmedium, SPcoord, TPcoord, omega, G, sigma11, sigma22, sigma33, sigma12, sigma31, sigma32)

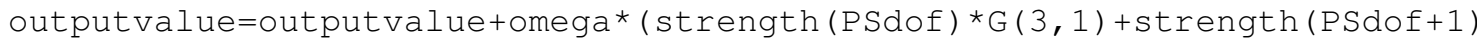
${ }^{\star} \mathrm{G}(3,2)+\&$

strength $(P \operatorname{Sdof}+2) * G(3,3))$

end select

endif

endif

end do

cpcounter $=$ cpcounter +1

end do

write $(1000, *) \mathrm{d} 1$, real (outputvalue), imag (outputvalue)

counter=counter +1

end do

close (1000)

end subroutine Plotline

! $* * * * * * * * * * * * *$ Function line Plot(it creates a line cross section

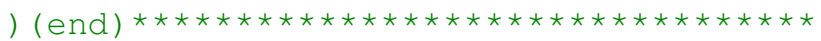

! $* \star \star \star * * * * * * * * *$ Function CircleMesh(it meshes a Circle and puts point

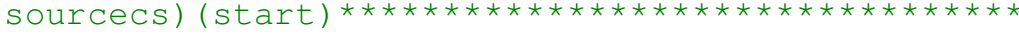

subroutine CircleMesh(Eobject, meshsize,PSradius, PSlist, LastPSlist)

type (circle) : : Fobject

real (8) : : PSradius, s, t, N (4), nx (3), ny (3), zunit (3), xp, yp, zp, xp2 (3), k, rloop real (8) : : meshsize, test,pi, beta

integer: : nump, nums, meshv, meshh, $i, j$, counter, Lastpslist, m, nloops, nloopps type (pointsource) : : PSlist (:)

$\mathrm{pi}=2 * \operatorname{acos}(0.0)$ 


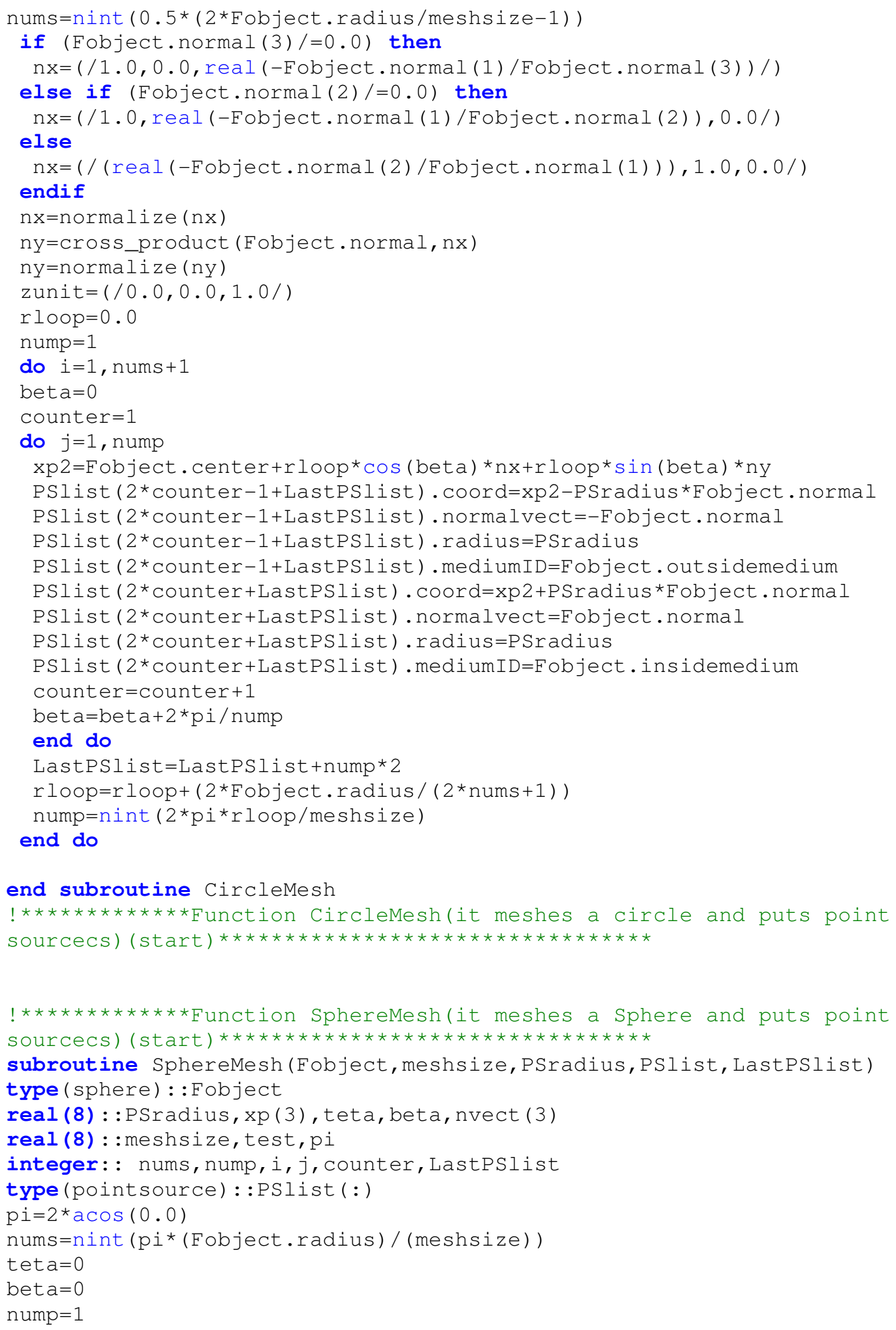




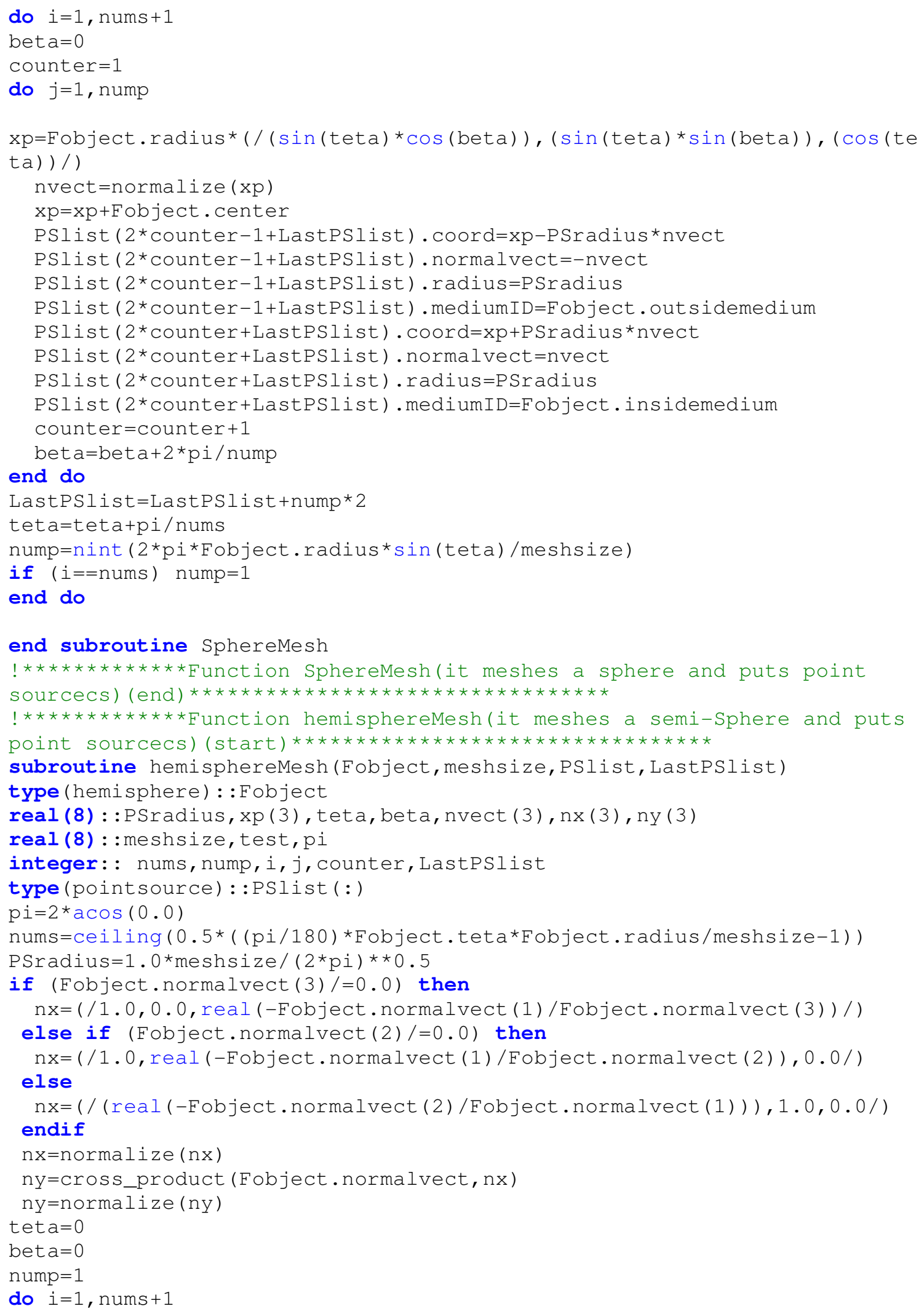




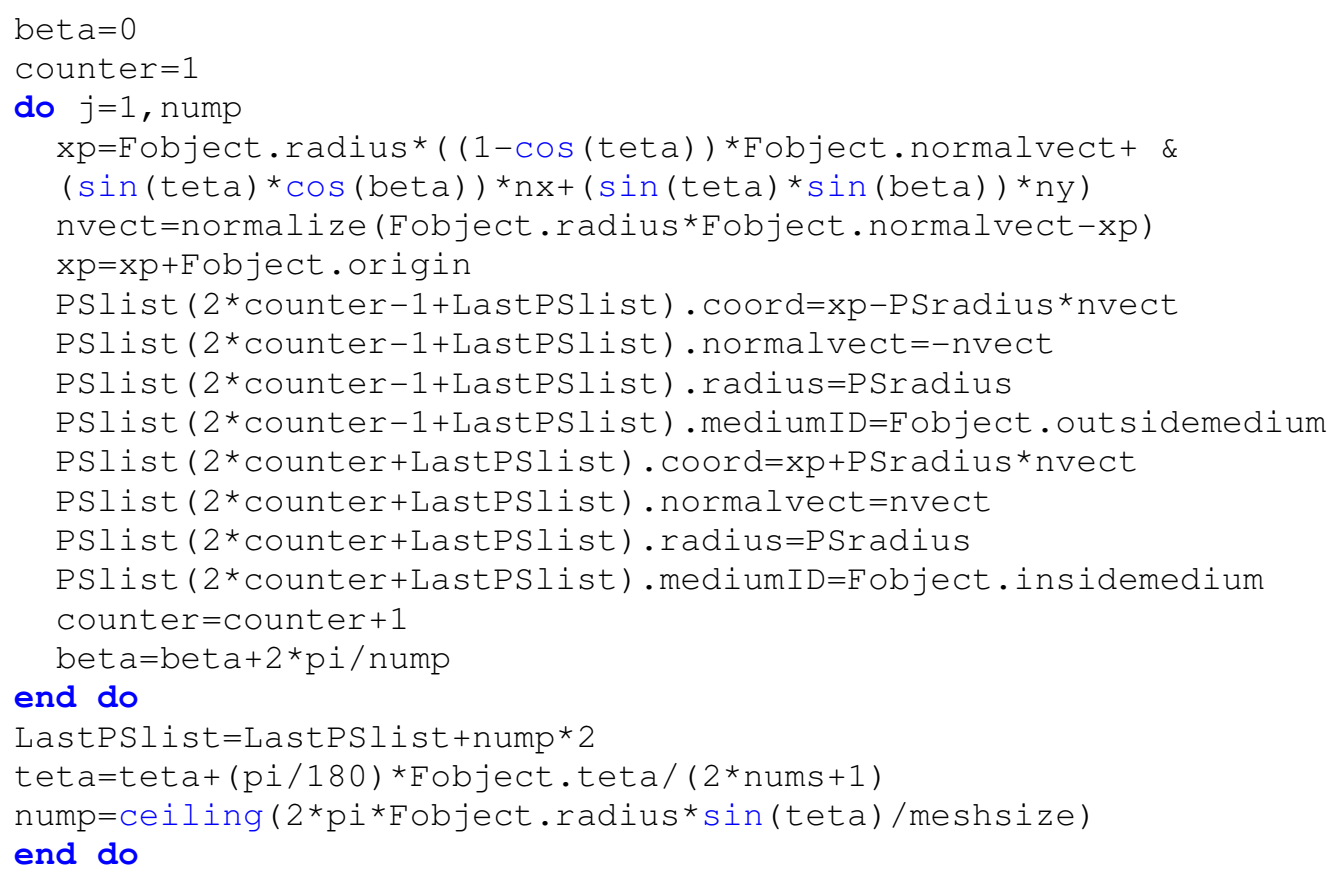

\section{function}

HemisphereEnergy (Objects, Fobject, Meshsize, DOFlist, Numcp, strength, Plotme dium, omega, outputcase)

type (hemisphere) : : Fobject

real (8) : : s, t, N (2), nz (3), nx (3), ny (3), sp,tp, Tpcoord (3), Spcoord (3), CPcoord (3) 


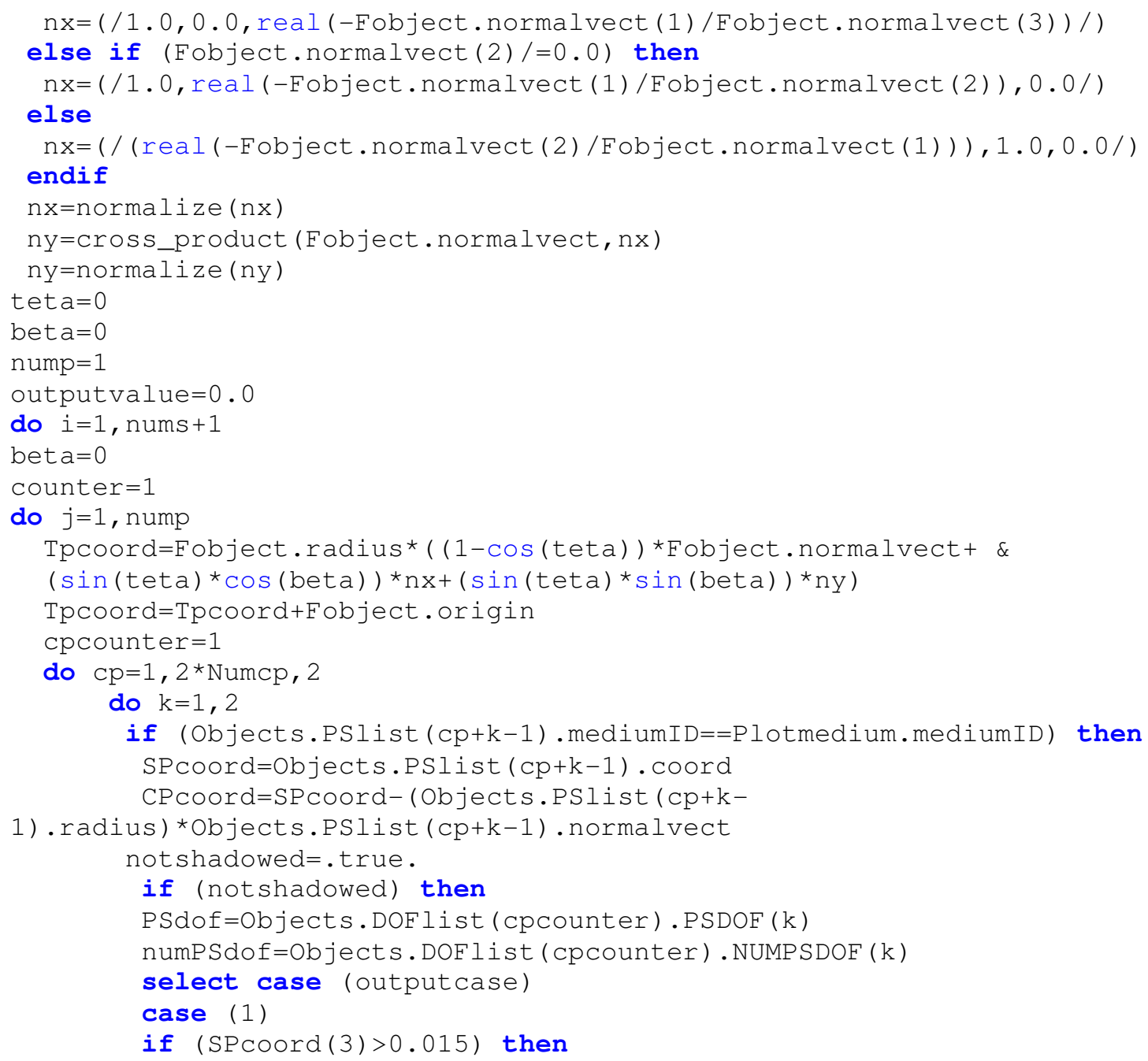




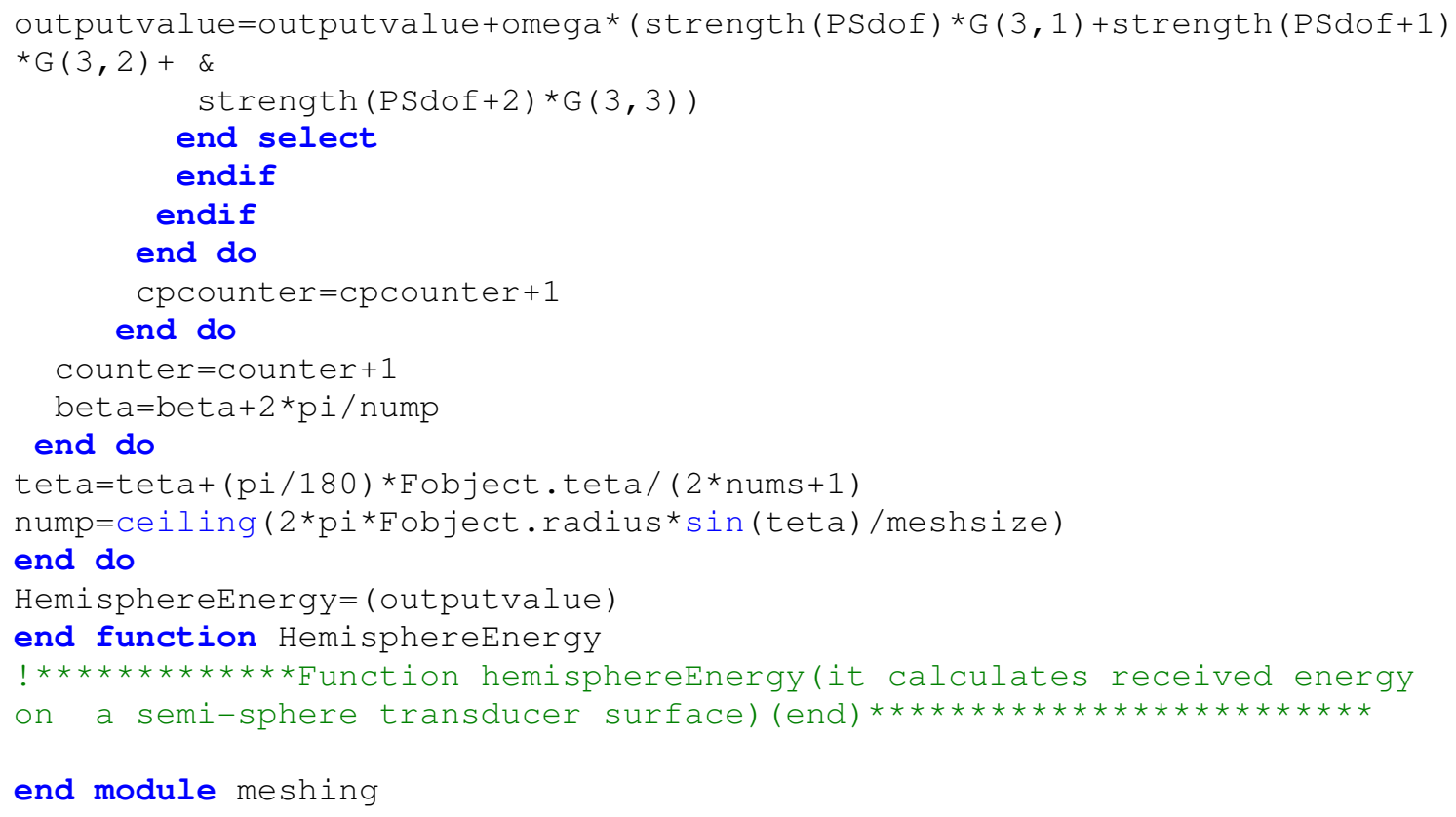




\section{"geometrylibrary" Module:}

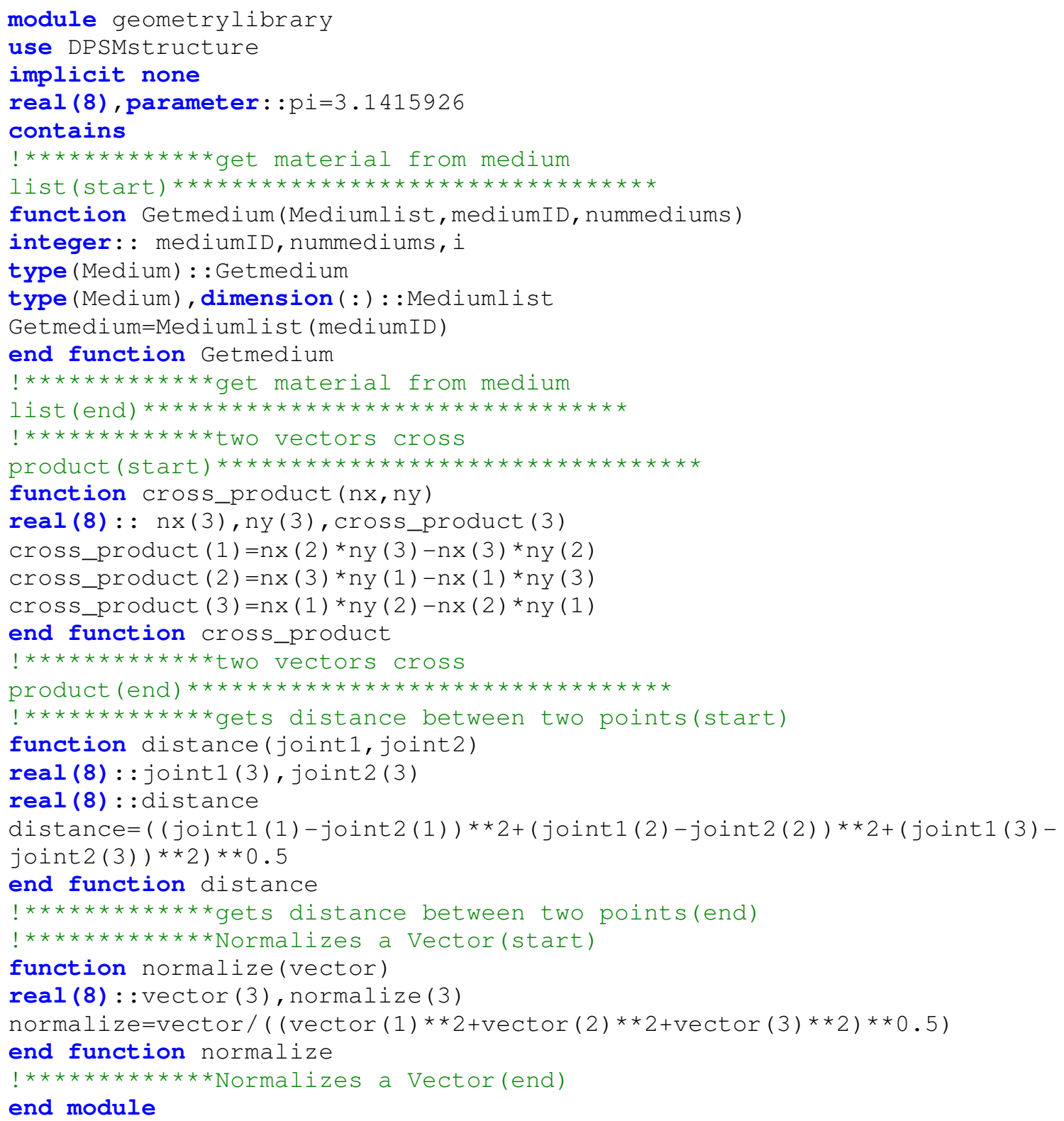


"Excitation" Module:

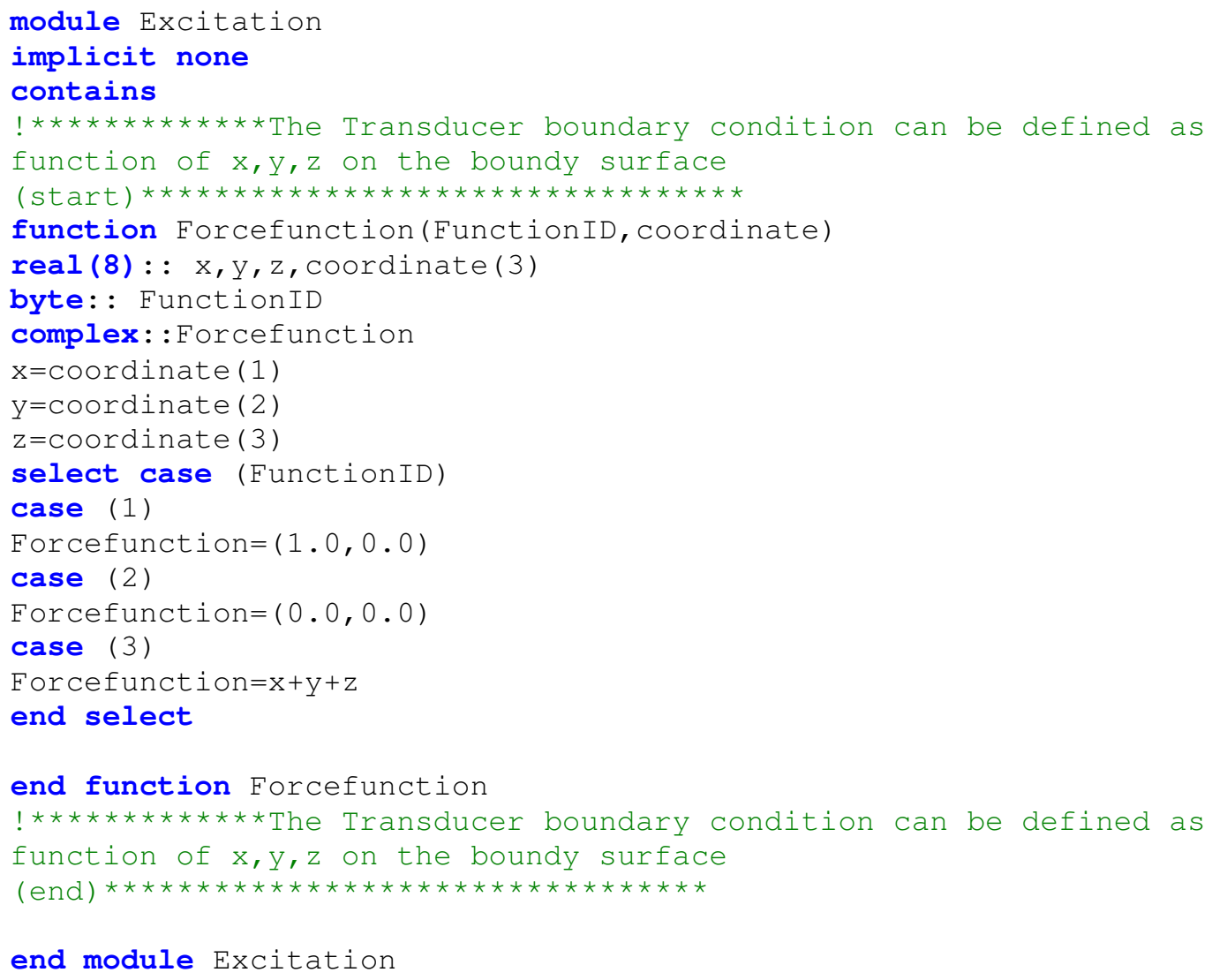




\section{"DPSMstructure" Module:}

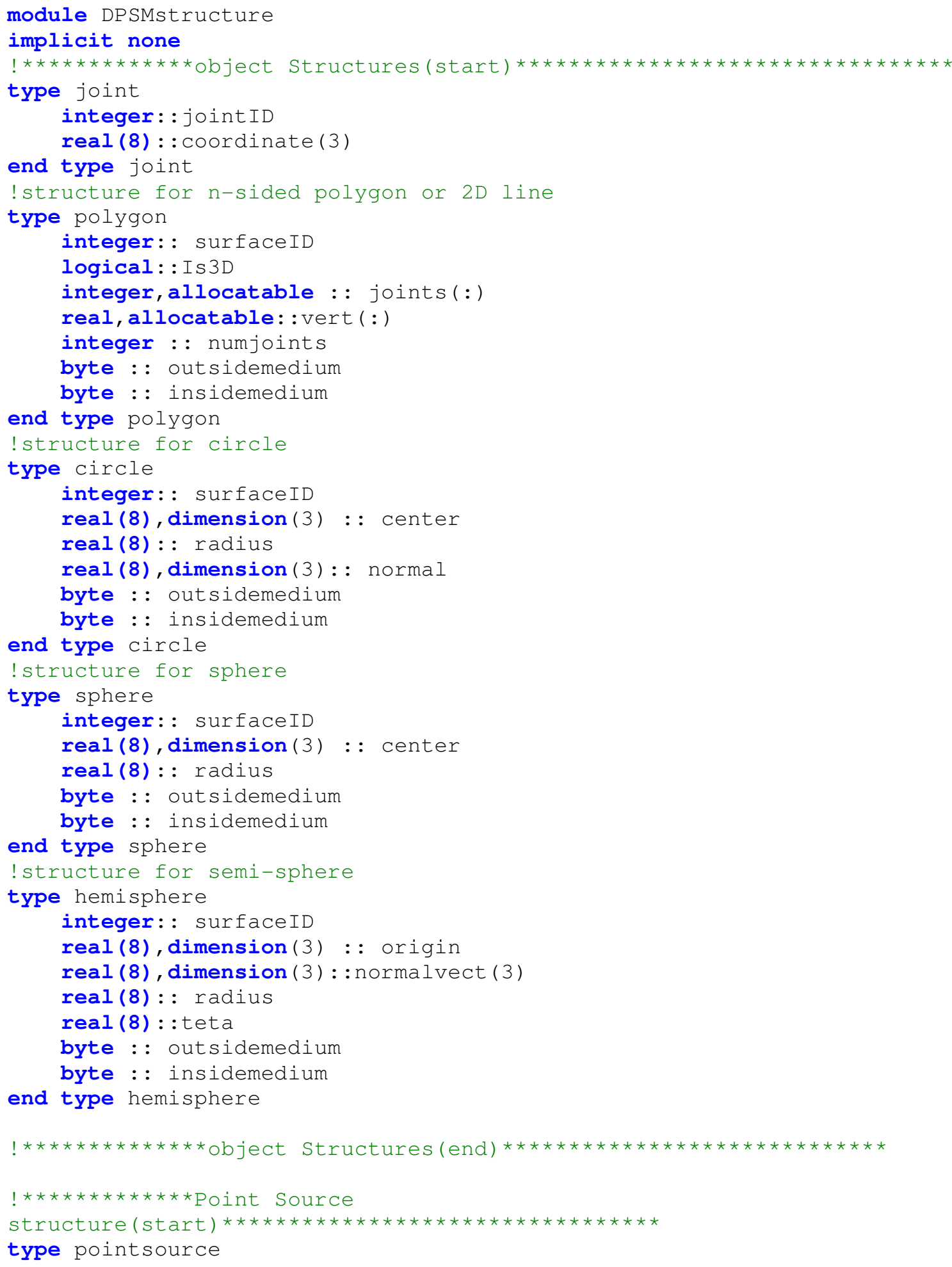




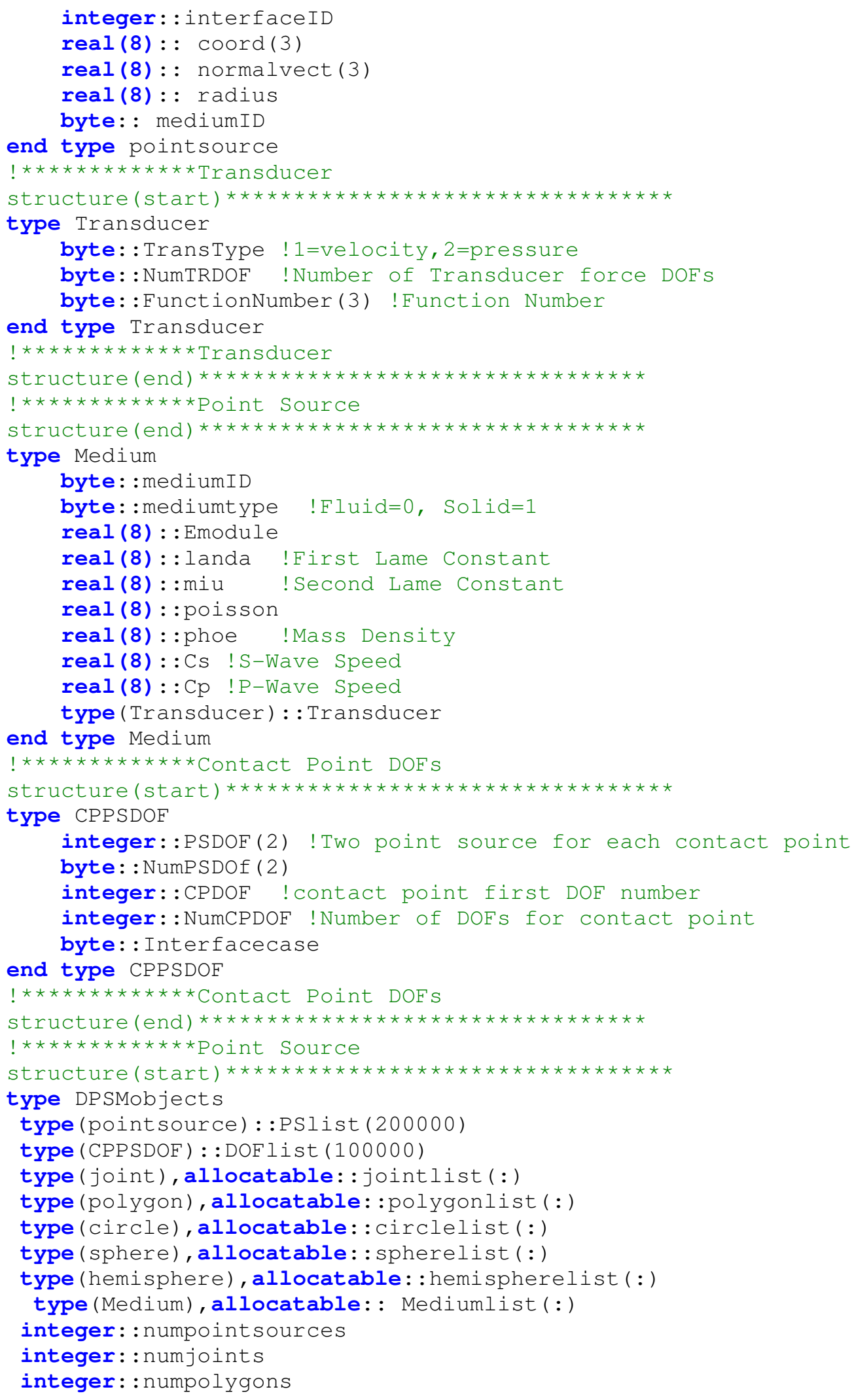


integer: : numcircles

integer: : numspheres

integer: : numhemispheres

integer: : nummediums

end type DPSMobjects

end module DPSMstructure 


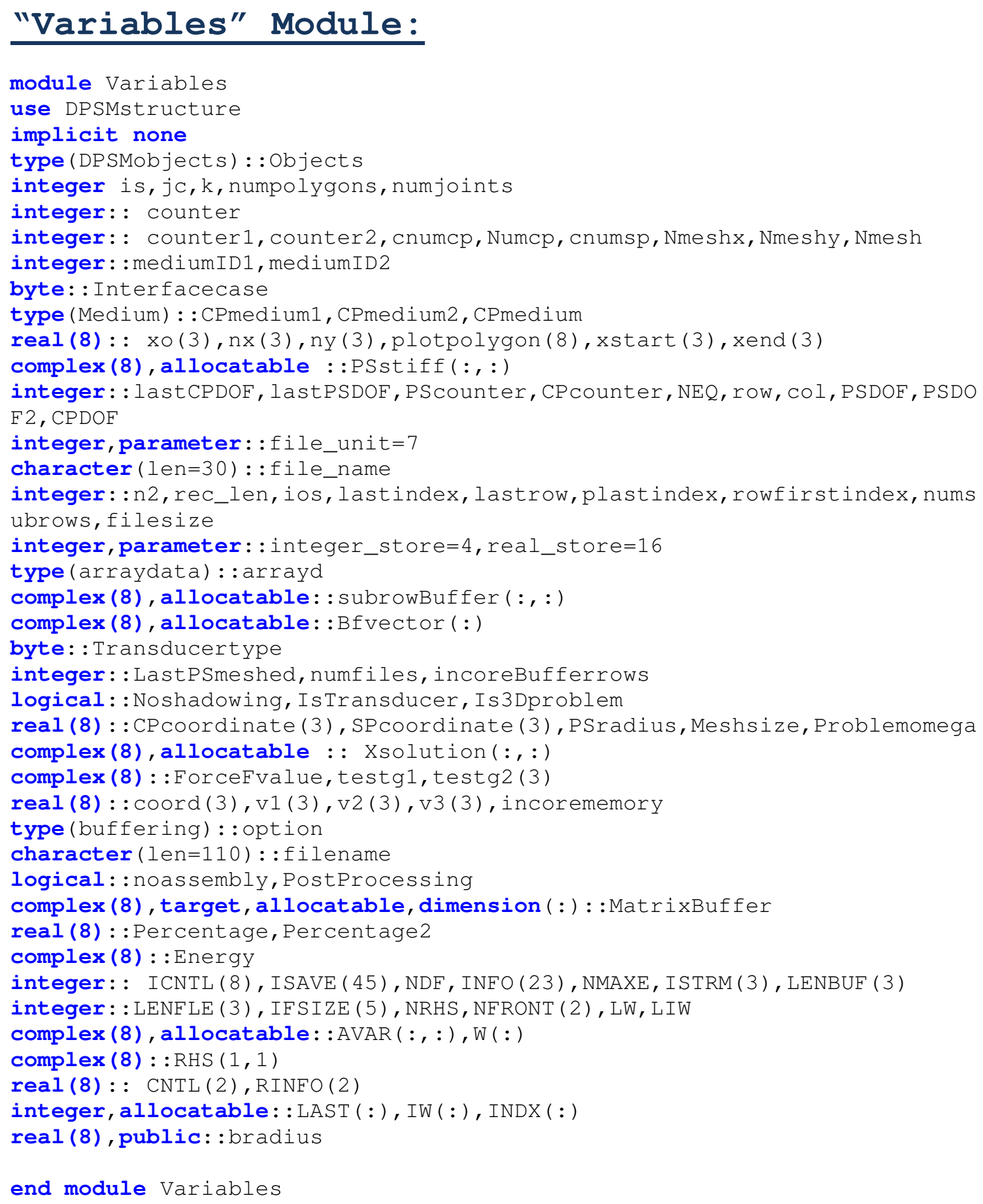




\title{
APPENDIX B: DPSM3D FORTRAN SOURCE CODE FOR ELECTROMAGNETIC PROBLEMS
}

\author{
Main Body of the code: \\ program DPSM_3d_Electromagnetics \\ use Variables \\ use geometrylibrary \\ use meshing \\ use greensfunctions \\ use Excitation \\ implicit none \\ external ME 42AD, ME42BD, ME 42CD, ME 42 ID, ME 42JD, ME 42PD

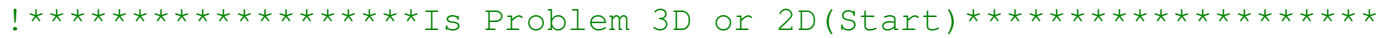 \\ Is 3Dproblem $=$. True.

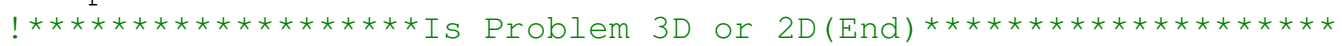

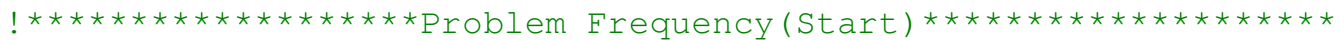 \\ Problemomega $=2 * \mathrm{pi} * 0.73 * 10 * \star 12.0$

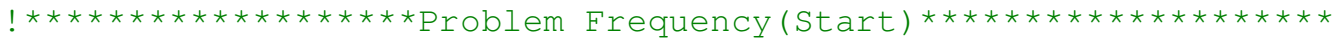

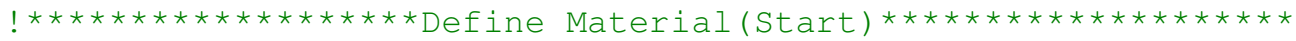 \\ Object $\mathrm{s}$. nummediums $=4$ \\ allocate (Objects. Mediumlist (Objects.nummediums)) \\ Objects.Mediumlist(1).mediumID=1 !Vaccume \\ Objects.Mediumlist (1).mediumtype=3 !Dielectric/EM=3 \\ Objects.Mediumlist ( 1$)$. Permittivity $=8.85 * 10 * *-12.0 *(1.0,0.0)$ \\ objects. Mediumlist ( 1$)$. Permeability $=4 * \mathrm{pi} * 10 * *-7.0 *(1.0,0.0)$ \\ Objects. Mediumlist ( 1$)$. conductivity $=0.0 *(1.0,0.0)$ \\ Objects.Mediumlist (1). vmedium=(1.0/(Objects.Mediumlist (1).Permittivity* \\ $\&$ \\ Objects.Mediumlist (1).Permeability)) ( 0.5 \\ Objects.Mediumlist (3) $\cdot$ mediumID=3 !bubble1 \\ Objects.Mediumlist ( 3 ). mediumtype=3 ! Dielectric/EM=3 \\ Objects. Mediumlist ( 3$)$. Permittivity $=(1.2-1.2 * 0.03 *(0.0,1.0)) * 8.85 * 10 * \star-$ \\ 12.0 \\ Objects.Mediumlist ( 3$)$. Permeability $=4 * \mathrm{pi} * 10 * *-7.0 *(1.0,0.0)$ \\ Objects.Mediumlist ( 3$)$. conductivity $=0.0 *(1.0,0.0)$ \\ Objects.Mediumlist (3). vmedium=(1.0/(Objects.Mediumlist (3). Permittivity* \\ $\&$ \\ Objects.Mediumlist ( 3 ).Permeability)) **0.5 \\ Objects.Mediumlist ( 4 ) . mediumID=4 !bubble2 \\ Objects.Mediumlist (4). mediumtype=3 ! Dielectric/EM=3
}




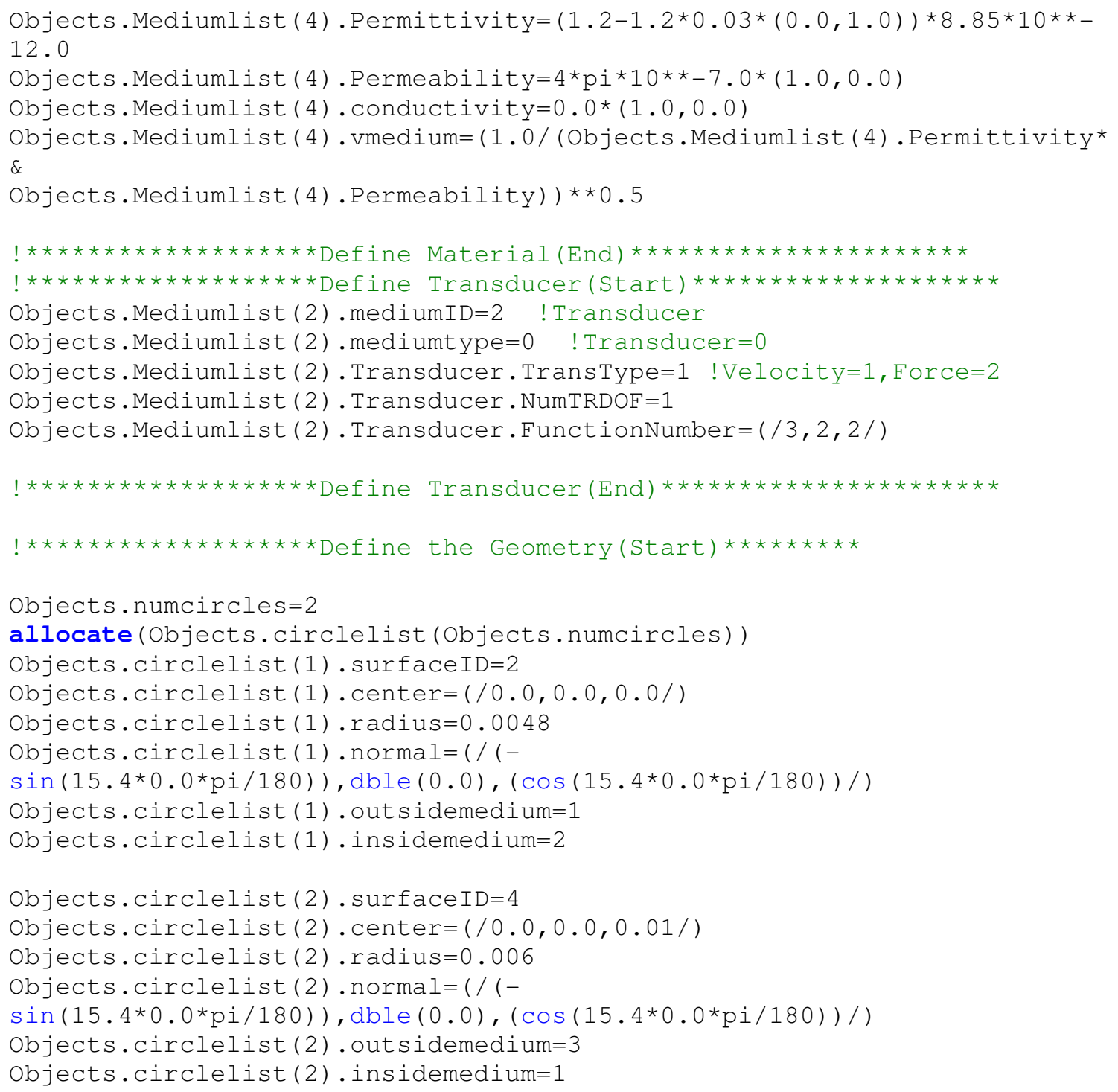

wt $=0.99 * 29 * 2 *$ (real (Objects.mediumlist (1). vmedium)/problemomega) objects . numjoints $=8$

allocate (objects.jointlist (objects.numjoints))

objects.jointlist $(1) \cdot$ joint $I D=1$

objects.jointlist (1). coordinate $=(/ \mathrm{dble}(-0.0048),(-\mathrm{wt} / 2), \mathrm{dble}(0.01) /)$

objects.jointlist (2) - jointID=2

objects.jointlist (2). coordinate $=(/ \mathrm{dble}(0.0048),(-\mathrm{wt} / 2), \mathrm{dble}(0.01) /)$

objects.jointlist (3) - jointID=3

Objects.jointlist (3). coordinate $=(/ \mathrm{dble}(0.0048),(+\mathrm{wt} / 2), \mathrm{dble}(0.01) /)$

objects.jointlist $(4) \cdot$ joint $I D=4$

objects.jointlist $(4)$. coordinate $=(/ \mathrm{dble}(-0.0048),(+\mathrm{wt} / 2), \mathrm{dble}(0.01) /)$

Objects.jointlist (5) - jointID=5

Objects.jointlist (5). coordinate $=(/ \mathrm{dble}(-0.0048),(-\mathrm{wt} / 2), \mathrm{dble}(0.0227) /)$

objects.jointlist $(6) \cdot$ joint ID $=6$ 


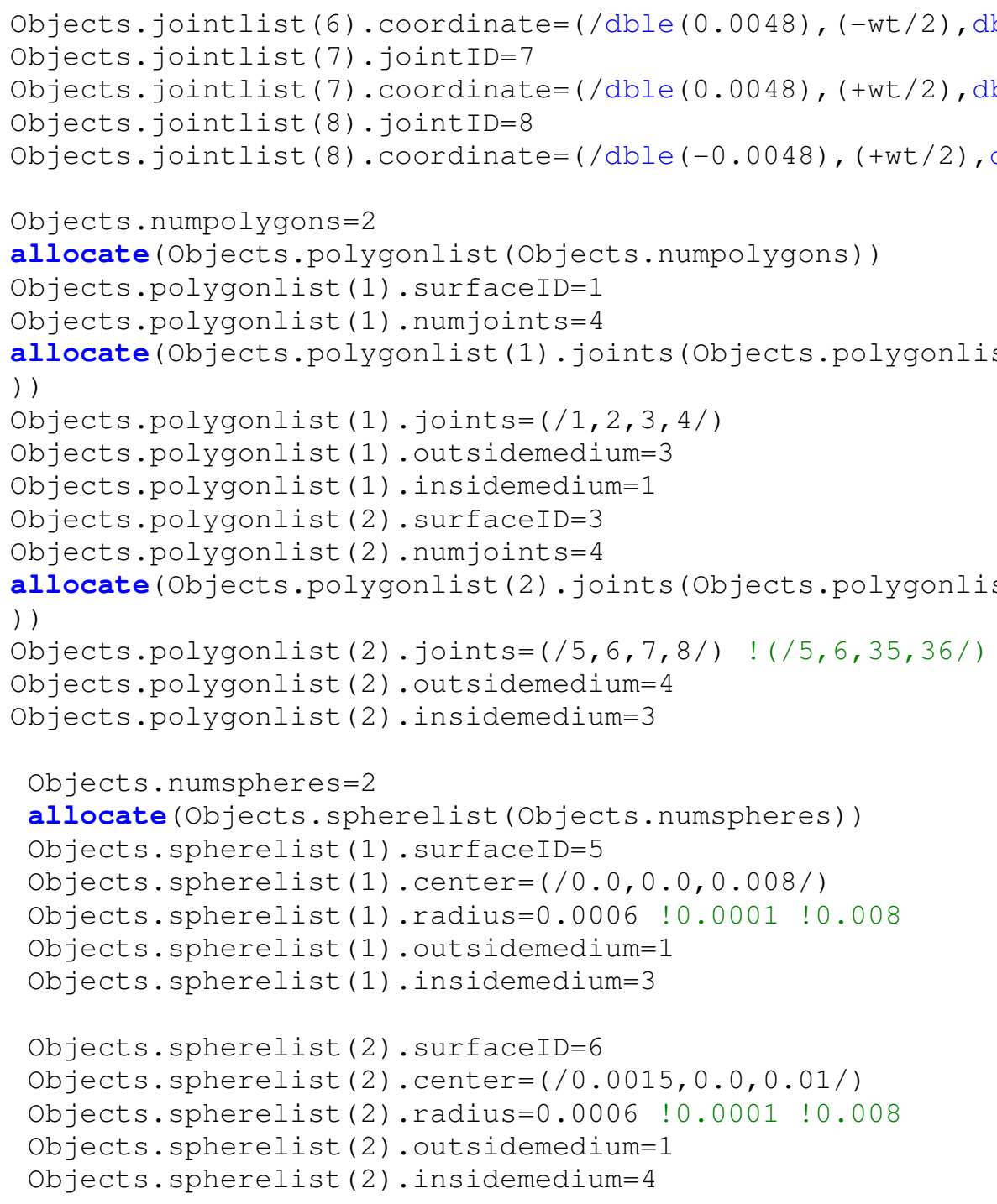

objects. numpolygons $=2$

objects . numcircles $=1$

objects . numhemispheres $=0$

objects . numspheres $=2$

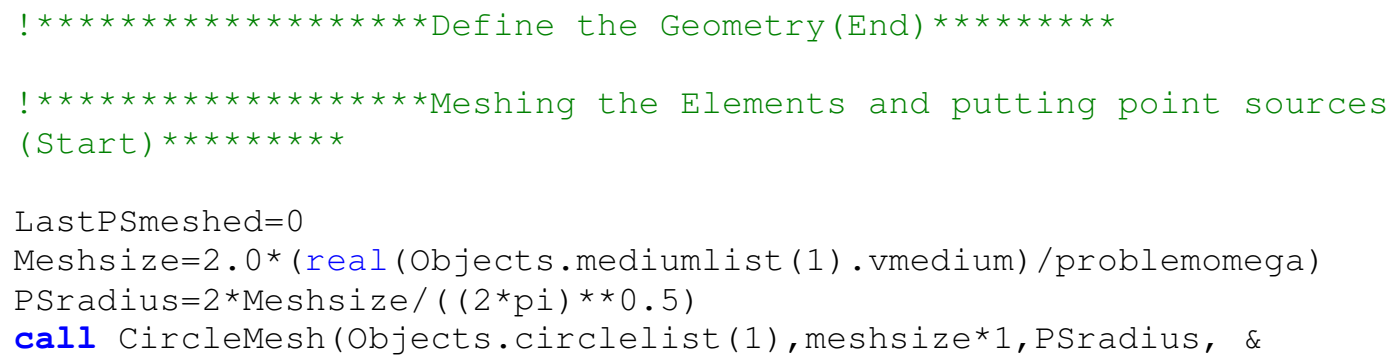


Objects.PSlist, LastPSmeshed)

!call PolygonMesh(objects.polygonlist(1),PSradius, Meshsize, \&

Objects.PSlist, Objects.jointlist, Objects.numjoints, LastPSmeshed, Is 3 Dpro

blem)

Meshsize $=(4 * \mathrm{pi} * 0.0006 * \star 2 / 600.0) * \star 0.5$

PSradius $=2 *$ Meshsize/ ( $2 * \mathrm{pi}) * \star 0.5)$

call Spheremesh(Objects.spherelist(1), meshsize,PSradius, \&

objects.PSlist, LastPSmeshed)

call SphereMesh(Objects.spherelist(2), meshsize,PSradius, \&

objects.PSlist, LastPSmeshed)

write $(*, *)$ LastPSmeshed/2

Objects . numpoint sources=LastPSmeshed

! $* * * * * * * * * * * * * * * * * * *$ Meshing the Elements and putting point sources

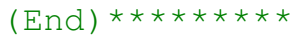

! **********Numbering (Start)

Numcp $=$ objects. numpoint sources $/ 2$

counter $=1$

last $\mathrm{PSDOF}=0$

last $\mathrm{CPDOF}=0$

do $\operatorname{cnumcp}=1,2 *$ Numcp, 2

mediumIDI=Objects.PSIist ( cnumcp) . mediumID

mediumID2=Objects.PSlist (cnumcp+1). mediumID

CPmedium1=Getmedium (Objects.Mediumlist, mediumID1, objects.nummediums)

CPmedium2=Getmedium (Objects.Mediumlist, mediumID2, objects.nummediums)

Interfacecase $=0$

if (CPmedium1.mediumtype==1. and. CPmedium2.mediumtype==1) then !Fluid-

Fluid Interface

objects.DOFlist (counter). Interfacecase $=1$

Objects. DOFlist (counter) $\cdot \mathrm{CPDOF}=$ last CPDOF+1

Objects.DOFlist ( counter) . numCPDOF $=2$

last $\mathrm{CPDOF}=$ last $\mathrm{CPDOF}+2$

Objects.DOFlist (counter). PSDOF ( 1 ) =lastPSDOF +1

Objects.DOFlist (counter). $\operatorname{PSDOF}(2)=1$ astPSDOF +2

Objects.DOFlist (counter) . numPSDOF $(1)=1$

objects.DOFlist (counter) $\operatorname{numPSDOF}(2)=1$

lastP $\mathrm{SDOF}=1$ astP $\mathrm{SDOF}+2$

\section{else if}

( ( CPmedium1. mediumtype==1. and. CPmedium2. mediumtype==2). or. ( CPmedium1.me diumtype $==2$. and. CPmedium2. mediumtype==1)) then !Fluid-Solid Interface

objects.DOFlist (counter). Interfacecase $=2$

Objects. DOFlist ( counter) $. \mathrm{CPDOF}=$ last $\mathrm{CPDOF}+1$

Objects. DOFlist ( counter) . numCPDOF $=4$

last $\mathrm{CPDOF}=$ last $\mathrm{CPDOF}+4$

if (CPmedium1.mediumtype==1) then

Objects.DOFlist (counter). PSDOF (1) =lastPSDOF +1

Objects.DOFlist (counter) . PSDOF (2) $=$ lastPSDOF +2

Objects. DOFlist (counter). numPSDOF $(1)=1$

objects.DOFlist (counter) $\operatorname{numPSDOF}(2)=3$

\section{else}

Objects. DOFlist (counter). PSDOF ( 1 ) =lastPSDOF +1

Objects.DOFlist (counter). $\operatorname{PSDOF}(2)=$ lastPSDOF +4

Objects.DOFlist (counter) $\operatorname{numPSDOF}(1)=3$

objects. DOFlist (counter) $\operatorname{numPSDOF}(2)=1$

end if 
last $\mathrm{PSDOF}=$ last $\mathrm{PSDOF}+4$

else if (CPmedium1.mediumtype==2. and. CPmedium2. mediumtype==2) then !Solid-Solid Interface

Objects.DOFlist (counter). Interfacecase $=3$

Objects. DOFlist (counter) $. \mathrm{CPDOF}=$ last $\mathrm{CPDOF}+1$

Objects. DOFlist (counter) . numCPDOF $=6$

last $\mathrm{CPDOF}=1$ ast $\mathrm{CPDOF}+6$

Objects.DOFlist (counter). $\operatorname{PSDOF}(1)=$ lastPSDOF +1

Objects.DOFlist (counter). $\operatorname{PSDOF}(2)=$ lastPSDOF +4

objects.DOFlist (counter) . numPSDOF $(1)=3$

objects.DOFlist (counter) $\operatorname{numPSDOF}(2)=3$

last $\mathrm{PSDOF}=$ lastP $\mathrm{SDOF}+6$

else if

( ( CPmedium1. mediumtype==1. and. CPmedium2. mediumtype==0) . or. ( CPmedium1.me diumtype $=0$. and. CPmedium2. mediumtype==1)) then ! Fluid-Transducer Interface

Objects.DOFlist (counter). Interfacecase=4 ! Fluid-Transducer interface $=4$

Objects. DOFlist ( counter) $. \mathrm{CPDOF}=$ last $\mathrm{CPDOF}+1$

Objects.DOFlist ( counter) . numCPDOF $=1$

last $\mathrm{CPDOF}=$ last $\mathrm{CPDOF}+1$

if (CPmedium1.mediumtype==1) then

Objects.DOFlist (counter). PSDOF (1) =lastPSDOF +1

Objects.DOFlist (counter) $\cdot \operatorname{PSDOF}(2)=0$

Objects.DOFlist (counter) . numPSDOF $(1)=1$

objects. DOFlist (counter) $\cdot \operatorname{numPSDOF}(2)=0$

else

Objects.DOFlist (counter) $\cdot \operatorname{PSDOF}(1)=0$

Objects.DOFlist (counter) $\cdot \operatorname{PSDOF}(2)=$ lastPSDOF +1

Objects.DOFlist (counter) $\operatorname{numPSDOF}(1)=0$

objects.DOFlist (counter) $\operatorname{numPSDOF}(2)=1$

end if

last $\mathrm{PSDOF}=$ lastP $\mathrm{SDOF}+1$

else if

( ( CPmedium1. mediumtype==2. and. CPmedium2. mediumtype==0). or. ( CPmedium1.me diumtype $==0$. and. CPmedium2.mediumtype==2)) then !Fluid-Transducer Interface

Objects.DOFlist (counter). Interfacecase=5 ! Solid-Transducer interface $=5$

Objects. DOFlist ( counter) $\cdot \mathrm{CPDOF}=$ last $\mathrm{CPDOF}+1$

Objects. DOFlist (counter) $\cdot$ numCPDOF $=3$

last $\mathrm{CPDOF}=$ last $\mathrm{CPDOF}+3$

if (CPmedium1.mediumtype==2) then

Objects.DOFlist (counter). PSDOF ( 1 ) =lastPSDOF +1

Objects.DOFlist (counter) $\cdot \operatorname{PSDOF}(2)=0$

Objects.DOFlist (counter) $\operatorname{numPSDOF}(1)=3$

Objects. DOFlist (counter). numPSDOF $(2)=0$

else

Objects.DOFlist (counter) $\cdot \operatorname{PSDOF}(1)=0$

Objects. DOFlist (counter). $\operatorname{PSDOF}(2)=$ lastPSDOF +1

Objects. DOFlist (counter) . numPSDOF $(1)=0$

Objects.DOFlist (counter) $\cdot \operatorname{numPSDOF}(2)=3$

end if

last $\mathrm{PSDOF}=$ last $\mathrm{PSDOF}+3$ 


\section{else if}

( ( CPmedium1. mediumtype==3. and. CPmedium2. mediumtype==0). or. (CPmedium1.me diumtype $=0$. and. CPmedium2. mediumtype==3)) then ! Fluid-Transducer Interface

Objects.DOFlist (counter). Interfacecase=6 !EM-Transducer interface $=6$ Objects. DOFlist (counter) $. \mathrm{CPDOF}=1$ ast $\mathrm{CPDOF}+1$

Objects.DOFlist ( counter) . numCPDOF=3

last $\mathrm{CPDOF}=$ last $\mathrm{CPDOF}+3$

if (CPmedium1.mediumtype==3) then

Objects.DOFlist (counter). PSDOF ( 1 ) =lastPSDOF +1

Objects.DOFlist (counter). PSDOF $(2)=0$

Objects.DOFlist (counter) . numPSDOF $(1)=3$

objects. DOFlist (counter) . numPSDOF $(2)=0$

\section{else}

Objects.DOFlist (counter). PSDOF $(1)=0$

Objects.DOFlist (counter) $\cdot \operatorname{PSDOF}(2)=$ lastPSDOF +1

objects. DOFlist (counter). numPSDOF $(1)=0$

Objects. DOFlist (counter) $\operatorname{numPSDOF}(2)=3$

\section{end if}

lastP $\mathrm{SDOF}=$ lastP $\mathrm{SDOF}+3$

else if (CPmedium1.mediumtype==3. and. CPmedium2.mediumtype==3) then

!Dielectric-Dielectric Interface

objects.DOFlist (counter). Interfacecase=7 ! Dielectric-Dielectric interface $=7$

Objects. DOFlist (counter) $. \mathrm{CPDOF}=$ last $\mathrm{CPDOF}+1$

Objects. DOFlist ( counter) . numCPDOF $=6$

last $\mathrm{CPDOF}=$ last $\mathrm{CPDOF}+6$

Objects.DOFlist (counter). PSDOF ( 1 ) =lastPSDOF +1

Objects.DOFlist (counter) $\cdot \operatorname{PSDOF}(2)=$ lastPSDOF +4

Objects.DOFlist (counter) . numPSDOF $(1)=3$

objects.DOFlist (counter) $\cdot \operatorname{numPSDOF}(2)=3$

lastPSDOF $=$ lastP $\mathrm{SDOF}+6$

end if

counter=counter +1

\section{end do}

! $* * \star * * * * * * *$ Numbering (End)

! $* * * \star * \star * * * *$ Assembly (Start)

PostProcessing $=$.false.

$\mathrm{NEQ}=$ last $\mathrm{CPDOF}$

write $(*, '(/ a, /,(6 f 10.3)) ')$ 'Number of Equations is:'

write $(*, *) \mathrm{NEQ}$

allocate (Bfvector (NEQ))

if (PostProcessing) goto 1300

counter $=1$

counter $2=1$

PScounter $=1$

CPcounter $=1$

lastindex $=1$

lastrow $=1$

plastindex $=1$

rowfirstindex $=1$

arrayd.rvalue $=0.0$

numfiles=ceiling (real ( $N E Q * N E Q) /($ option.numfilerecs*option.reclength))

Bfvector $=0.0$ 


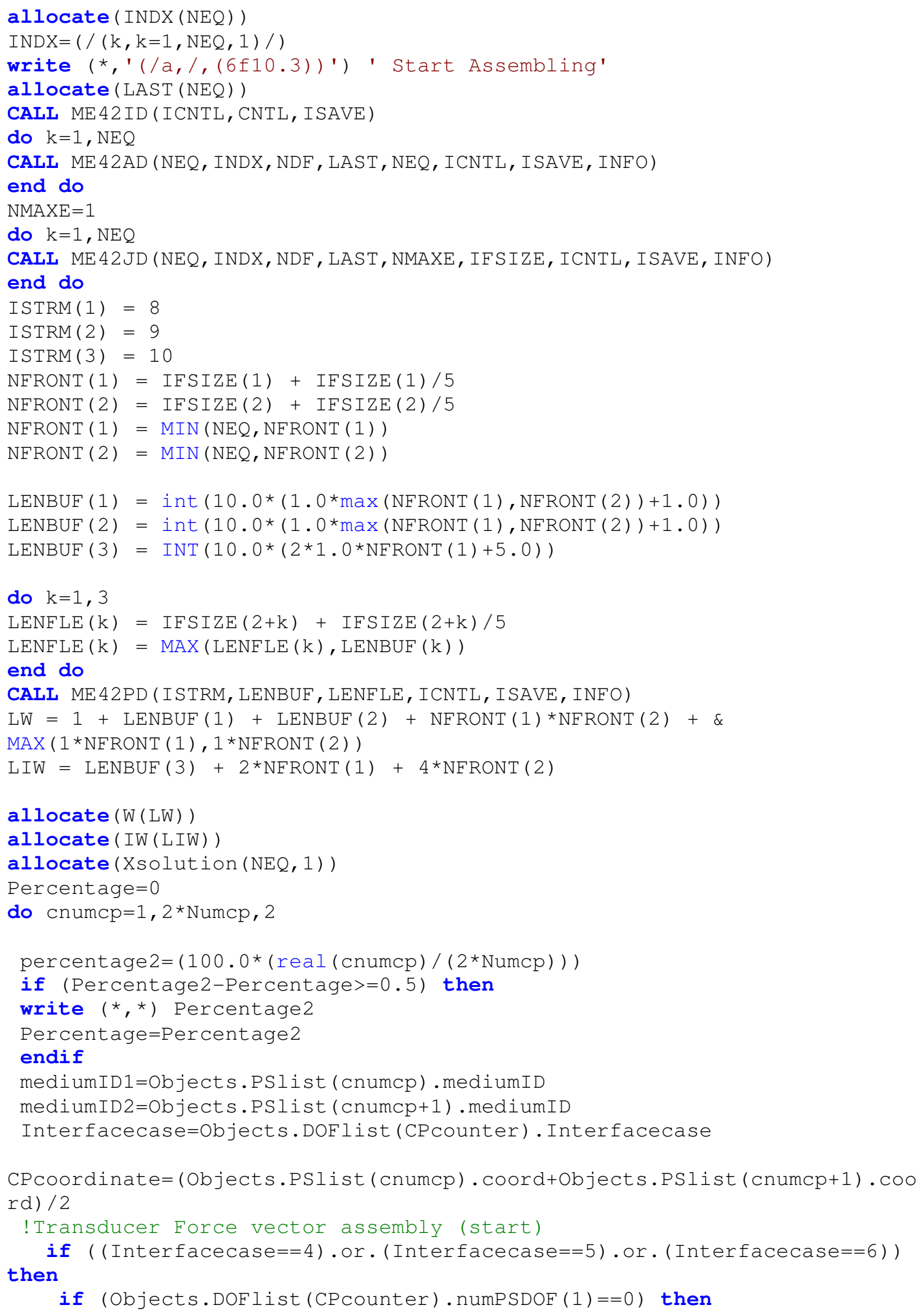




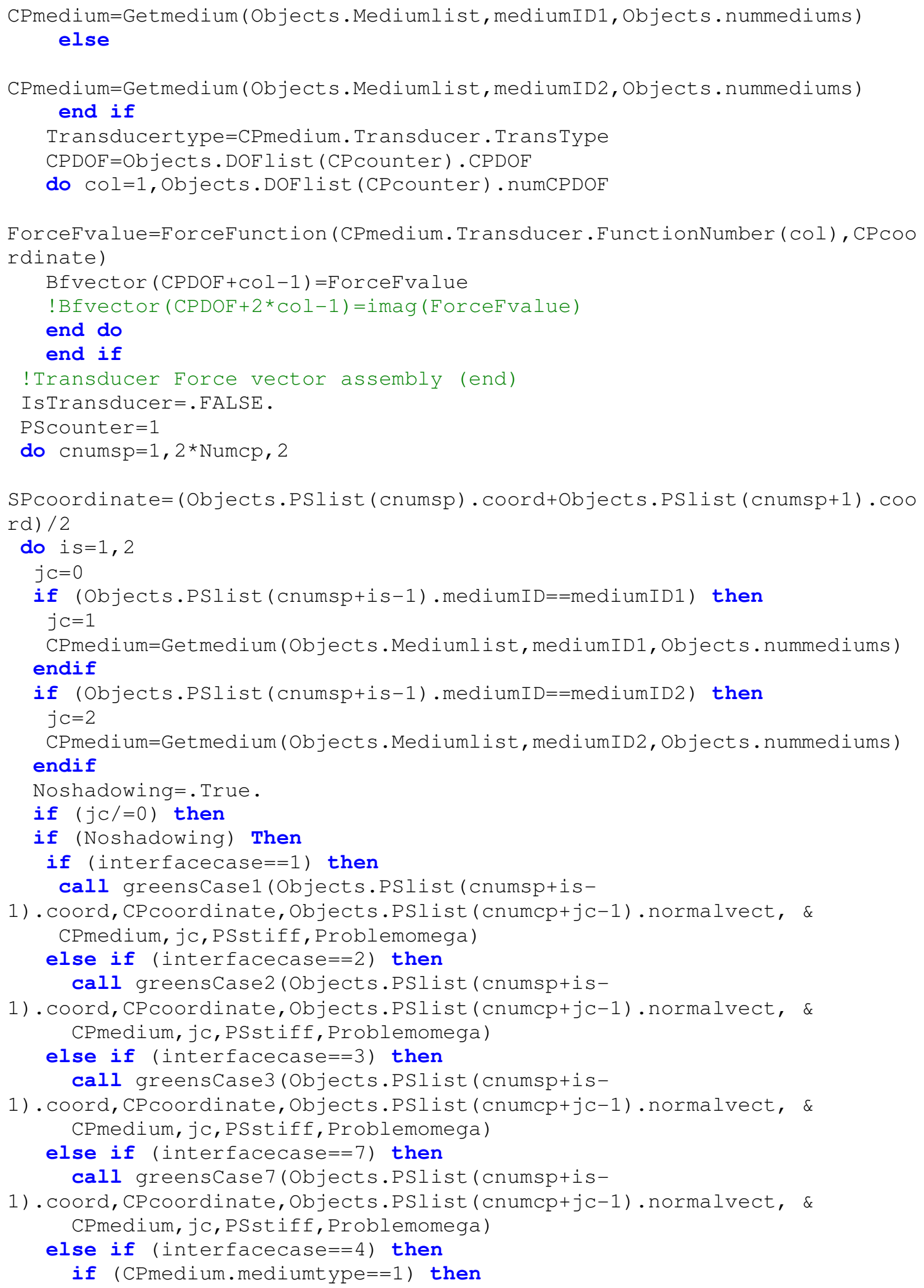


call greensCase4(Objects.PSlist (cnumsp+is-

1). coord, CPcoordinate, Objects.PSlist (cnumcp+jc-1).normalvect, \&

CPmedium, jc, PSstiff, Problemomega, Transducertype)

IsTransducer $=$. FALSE.

else

IsTransducer $=$. TRUE.

end if

else if (interfacecase $==5$ ) then

if (CPmedium.mediumtype==2) then

call greenscase5(Objects.PSlist (cnumsp+is-

1). coord, CPcoordinate, objects.PSlist (cnumcp+jc-1).normalvect, \&

CPmedium, jc, PSstiff, Problemomega, Transducertype)

IsTransducer $=$. FALSE.

else

IsTransducer $=$. TRUE.

end if

else if (interfacecase $==6$ ) then

if (CPmedium.mediumtype==3) then

call greenscase6 (Objects.PSlist (cnumsp+is-

1 ). coord, CPcoordinate, Objects.PSlist (cnumcp+jc-1).normalvect, \&

CPmedium, jc, PSstiff, Problemomega, Transducertype)

IsTransducer $=$.FALSE.

else

IsTransducer $=$. TRUE .

end if

endif

if (.not.IsTransducer) then

PSDOF=Objects. DOFlist (PScounter). PSDOF (is)

numsubrows=size (PSstiff, 1$)$

if (.not.allocated (subrowBuffer)) then

allocate (subrowBuffer (numsubrows, NEQ))

subrowBuffer $=0.0$

endif

subrowBuffer (:,PSDOF :PSDOF+size (PSstiff, 2)-1)=PSstiff

end if

endif !shadowing check if

end if !end for "if $j /="$

end do !for $i=1,2$

PScounter $=$ PScounter +1

end do

do $\mathrm{k}=1$, numsubrows

$\operatorname{RHS}(1,1)=$ Bfvector $($ lastrow $+\mathrm{k}-1)$

CALL ME42BD (NEQ, INDX, NDF, LAST, NMAXE, subrowBuffer $(k: k,:), 1, \operatorname{RHS}, 1, \mathrm{NEQ}, \&$

Xsolution, NFRONT, LENBUF, LW, W, LIW, IW, ICNTL, CNTL, ISAVE, INFO, RINFO)

end do

deallocate (subrowBuffer)

lastrow=lastrow +numsubrows

CPcounter $=$ CPcounter +1

end do

deallocate (W)

deallocate (IW) 


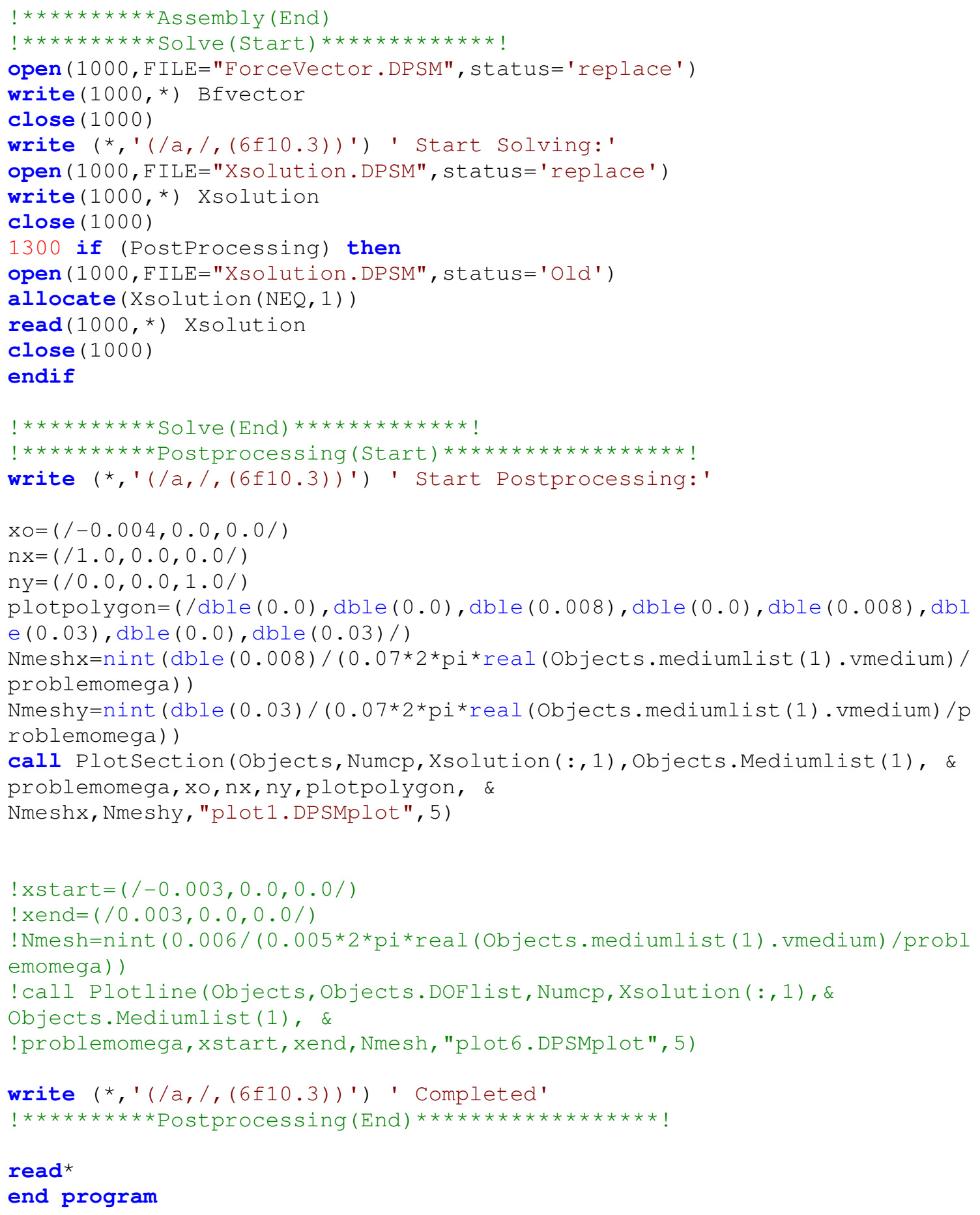




\section{"Greensfunctions" Module:}

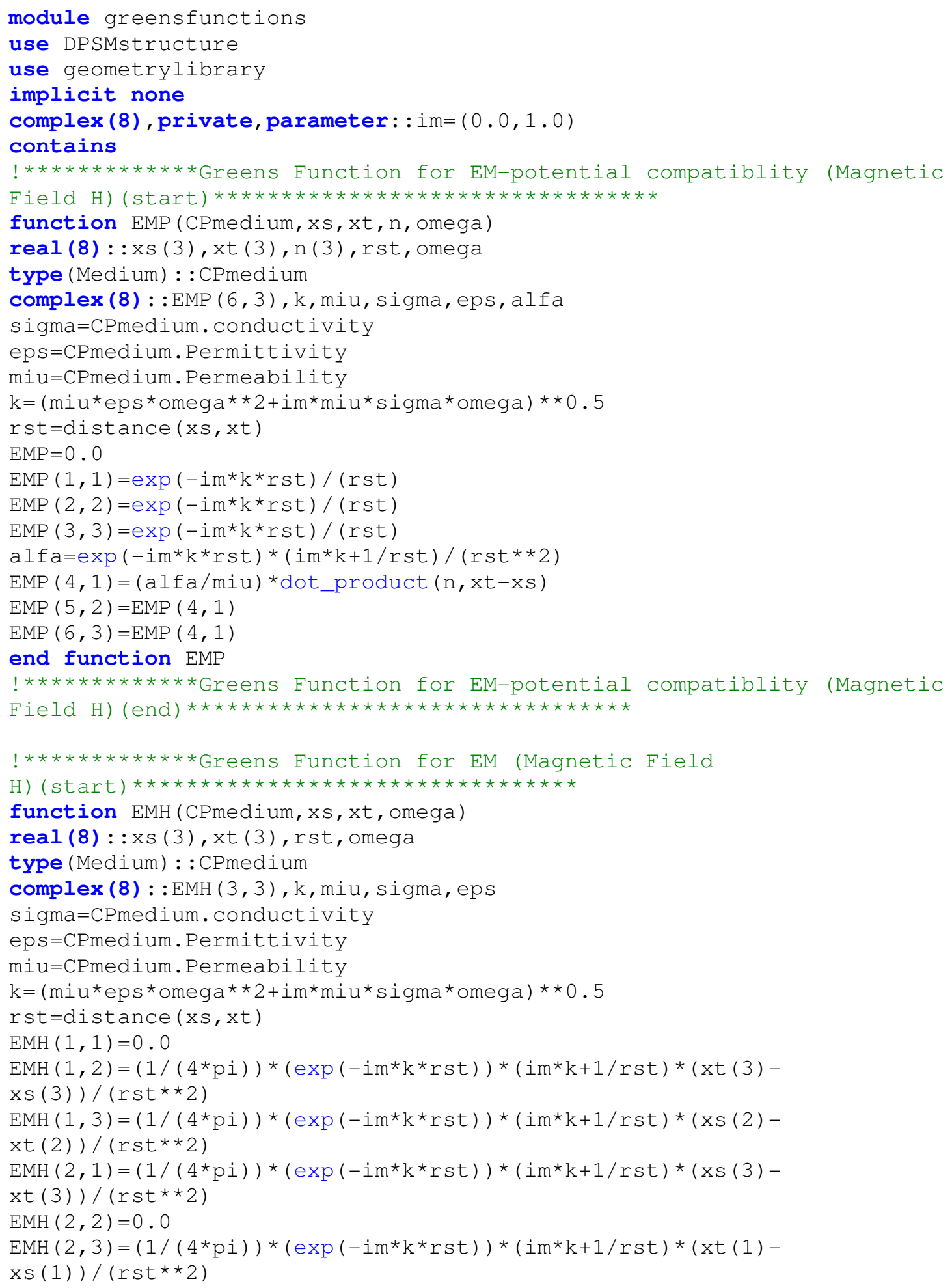




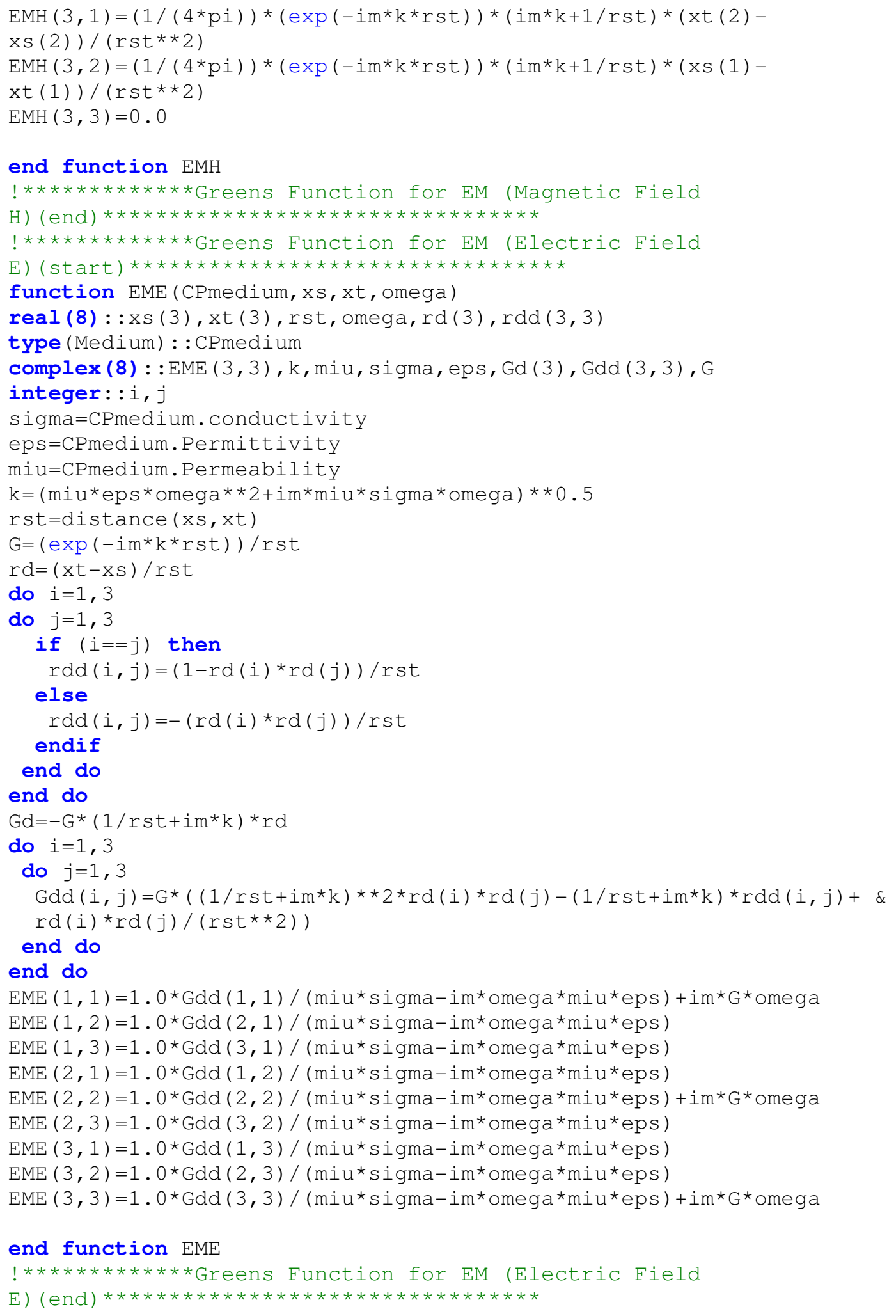




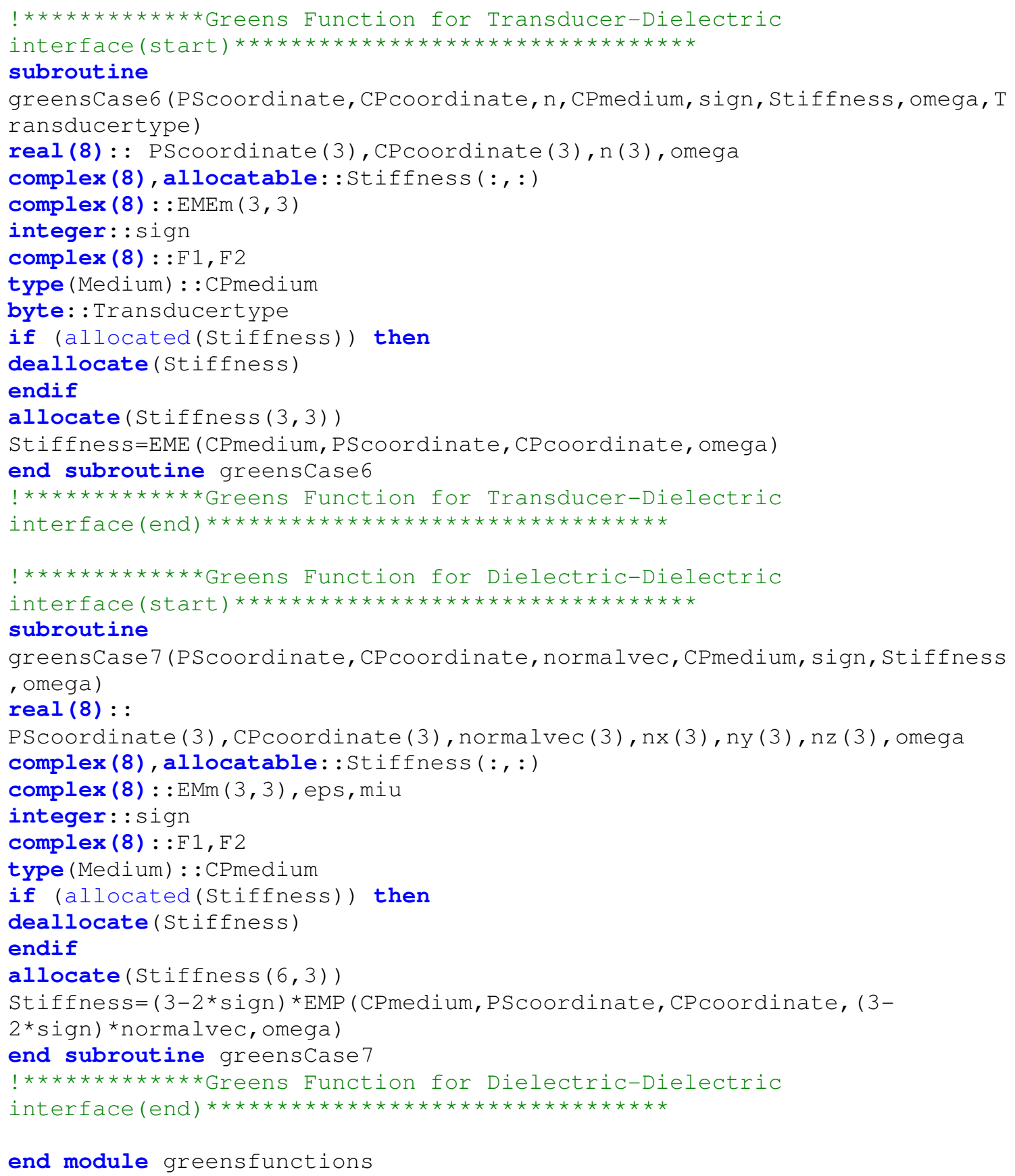




\section{"Meshing" Module:}

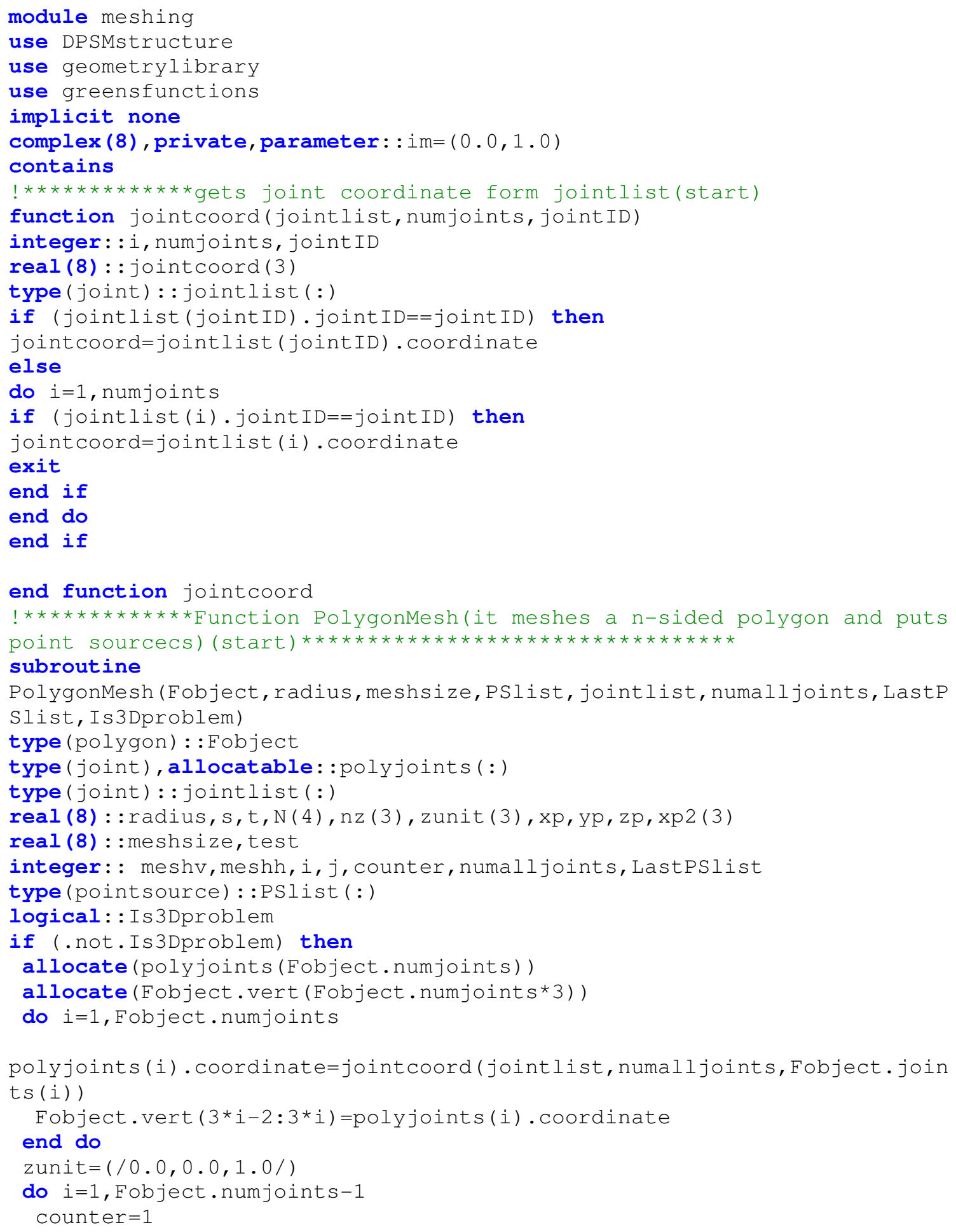




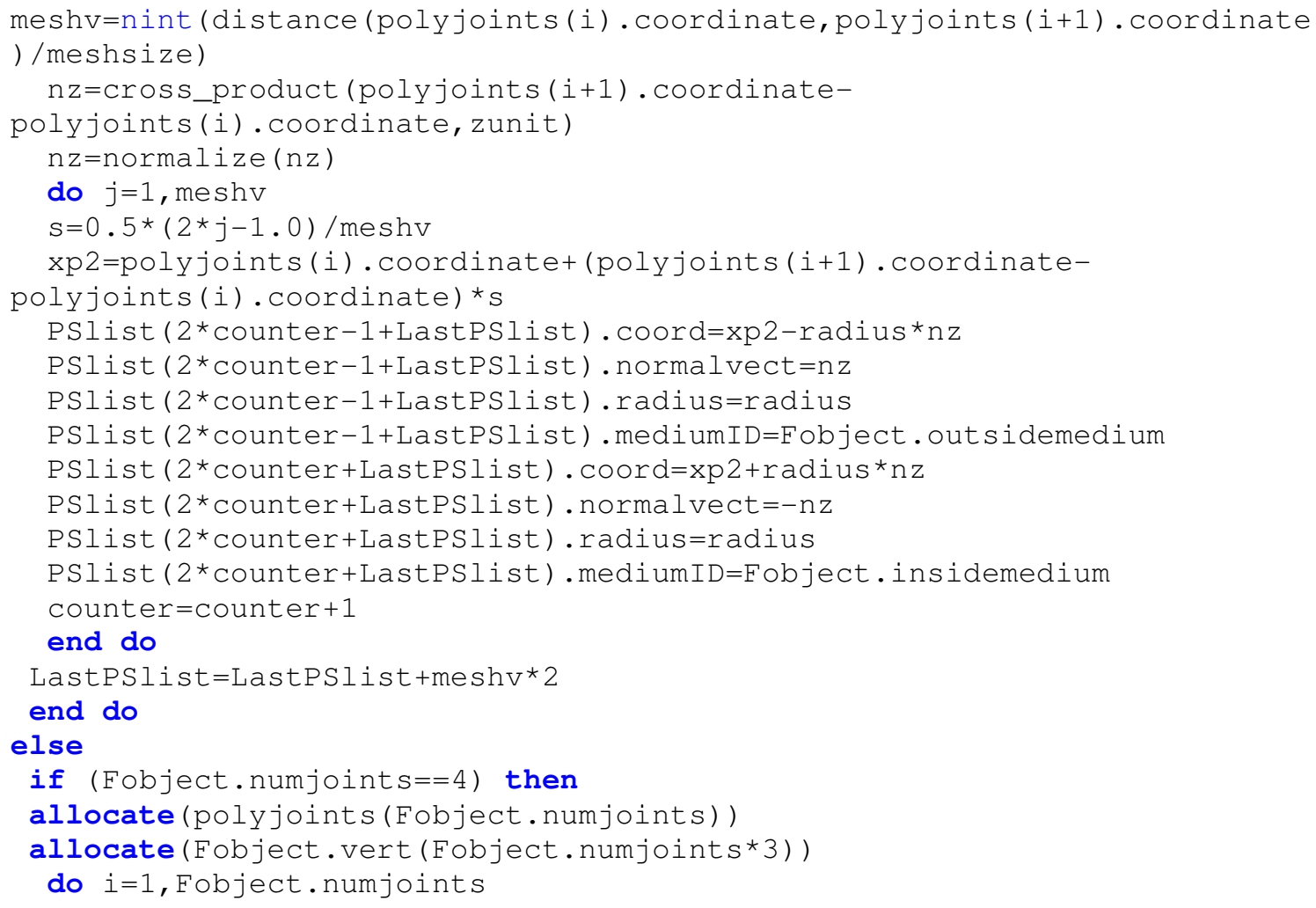

meshh=int (max (distance (polyjoints (1). coordinate, polyjoints (2). coordinat e), \&

distance (polyjoints (3). coordinate, polyjoints (4). coordinate))/(2*meshsiz e)) ! jointlist( 1 ).jointID

meshv=int (max (distance (polyjoints (2). coordinate, polyjoints (3). coordinat e), \&

distance (polyjoints (1). coordinate, polyjoints (4). coordinate))/(2*meshsiz e)) ! jointlist(1).jointID

meshh $=2 *$ meshh +1

meshv $=2 *$ meshv +1

! meshv=meshsize

! meshh=meshsize

! x=polyjoints $(1: 4)$. coordinate (1)

$! y=$ Fobject.points $(2: 8: 2)$ 
nz=cross_product (polyjoints (2) . coordinatepolyjoints (1) . coordinate, polyjoints (4). coordinatepolyjoints (1). coordinate)

$\mathrm{nz}=$ normalize (nz)

counter $=1$

do $i=1$, meshh

do $j=1$, meshv

$s=1.0-(2.0 * i-1.0) / \mathrm{meshh}$

$t=-1.0+(2.0 * j-1.0) / m e s h v$

$N(1)=0.25 *(1.0-s) *(1.0-t)$

$N(2)=0.25 *(1.0+s) *(1.0-t)$

$\mathrm{N}(3)=0.25 *(1.0+s) *(1.0+t)$

$N(4)=0.25 *(1.0-s) *(1.0+t)$

$\mathrm{xp}=$ dot_product $(\mathrm{N}$, polyjoints $(1: 4)$. coordinate (1))

$y p=d o t \_p r o d u c t(N$, polyjoints $(1: 4)$. coordinate (2))

$\mathrm{zp}=$ dot_product $(\mathrm{N}$, polyjoints $(1: 4)$. coordinate (3))

PSlist ( $2 *$ counter $-1+$ LastPSlist) . coord $=(/ \mathrm{xp}, \mathrm{yp}, \mathrm{zp} /)$-radius*nz

PSlist $(2 *$ counter $-1+$ LastPSlist $)$. normalvect $=-n z$

PSlist $(2 *$ counter $-1+$ LastPSlist $)$.radius=radius

PSlist ( $2 *$ counter $-1+$ LastPSlist) . mediumID $=$ Fobject . outsidemedium

PSlist ( ${ }^{*}$ counter+LastPSlist) $. \operatorname{coord}=(/ \mathrm{xp}, \mathrm{yp}, \mathrm{zp} /)+$ radius*nz

PSlist $(2 *$ counter+LastPSlist) . normalvect $=n z$

PSlist $(2 *$ counter+LastPSlist $)$.radius=radius

PSlist ( $2{ }^{*}$ counter+LastPSlist) . mediumID=Fobject. insidemedium

counter $=$ counter +1

end do

end do

LastPSlist $=$ LastPSlist $+($ meshh $) *($ meshv $) * 2$

else

!Triangle Meshing should be added here

end if

endif

if (allocated(polyjoints)) deallocate(polyjoints)

end subroutine PolygonMesh

! $* \star * \star * * * * * * * * *$ Function PolygonMesh(it meshes a n-sided polygon and puts

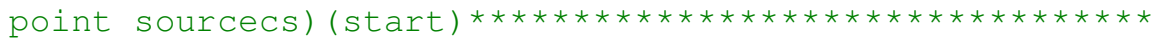

! $* * * * * * * * * * * * *$ Function PlotMeshgrid(it meshes a 4-sided polygon and

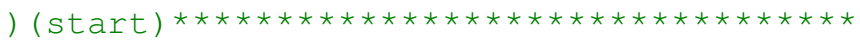

subroutine

PlotSection(Objects, Numcp, strength, Plotmedium, omega, xo, nx, ny, polygon, Nm eshx, Nmeshy, file_name, outputcase)

real (8): :s, t, N (4), nz(3), nx (3), ny (3), sp, tp, Tpcoord (3), Spcoord (3), CPcoord (3), xo (3), polygon (8)

real (8) : : test, d1, d2, d3, d4

character $(l e n=\star):$ : file_name

complex (8) : : outputvalue, FV2 (3), im2

type (Medium) : :Plotmedium

type (DPSMobjects) : : Objects

real (8) : : omega, tolerance

complex (8): :strength (:) 


\section{integer: :}

m, Nmeshx, Nmeshy, outputcase, meshs, mesht, i, j, k, cp, counter, cpcounter, Numcp , PSdof, numPSdof

logical: : notshadowed

complex (8) : : G (3,3), sigma33 (3), sigma11 (3), sigma22(3), sigma31(3), sigma12( 3), sigma32(3)

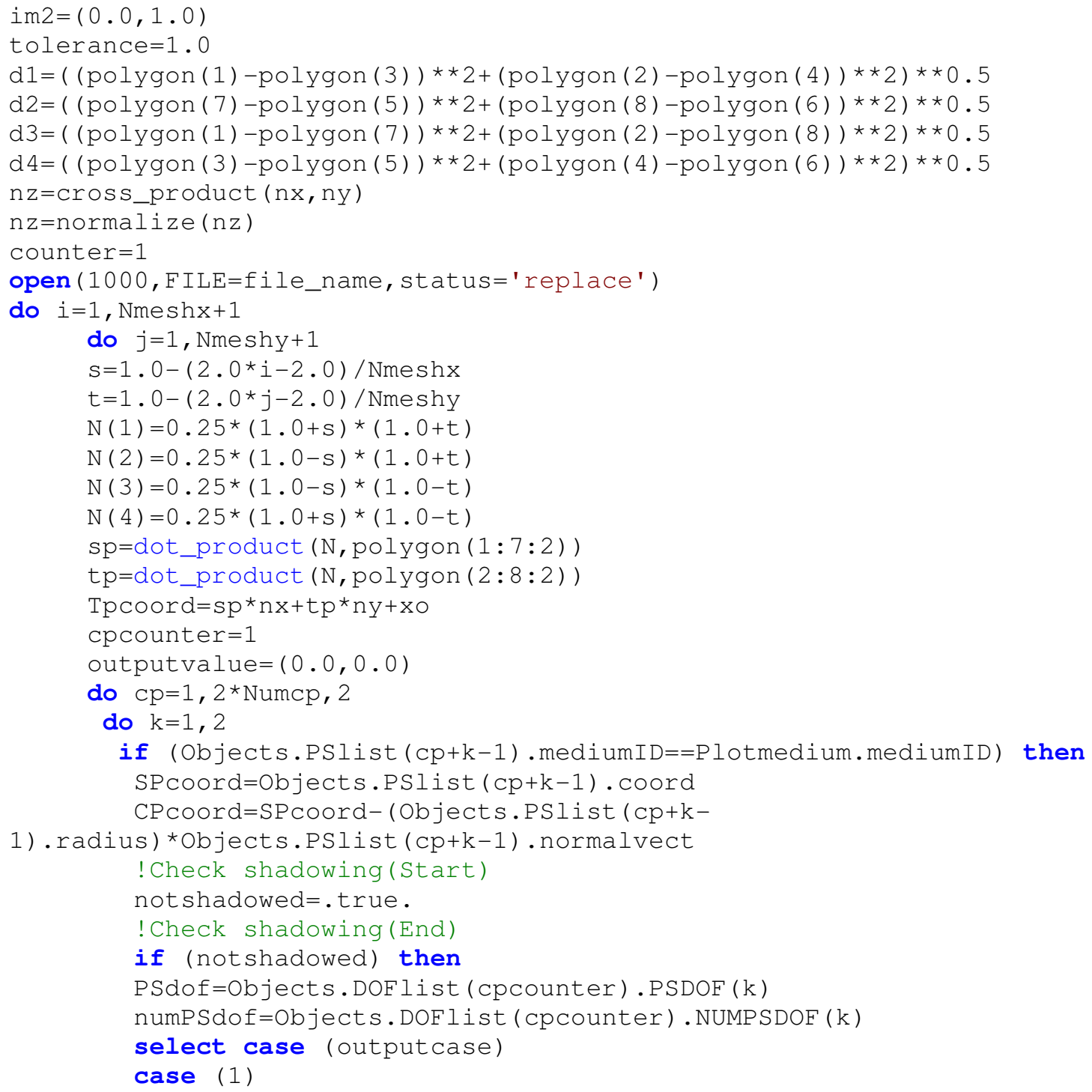




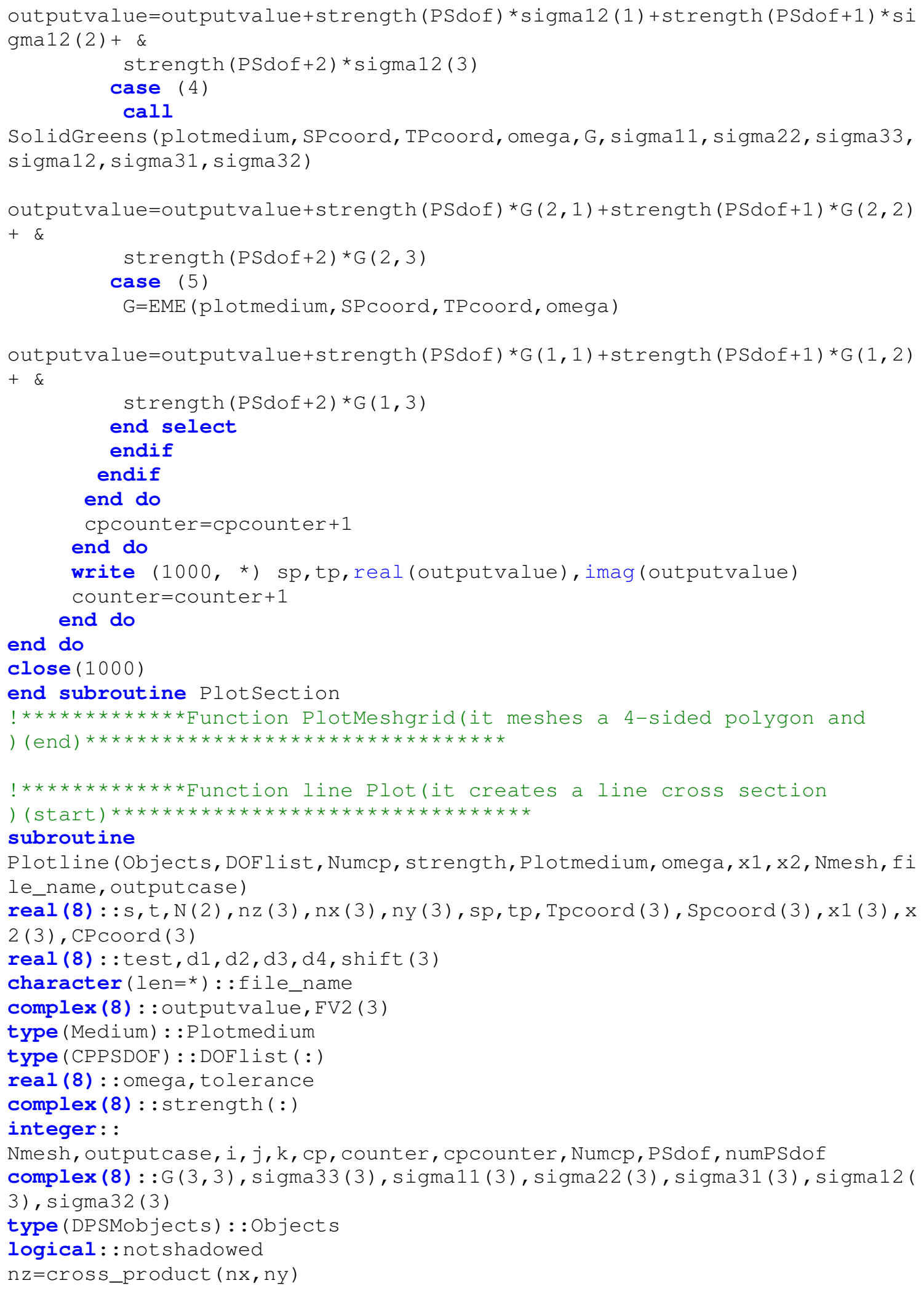




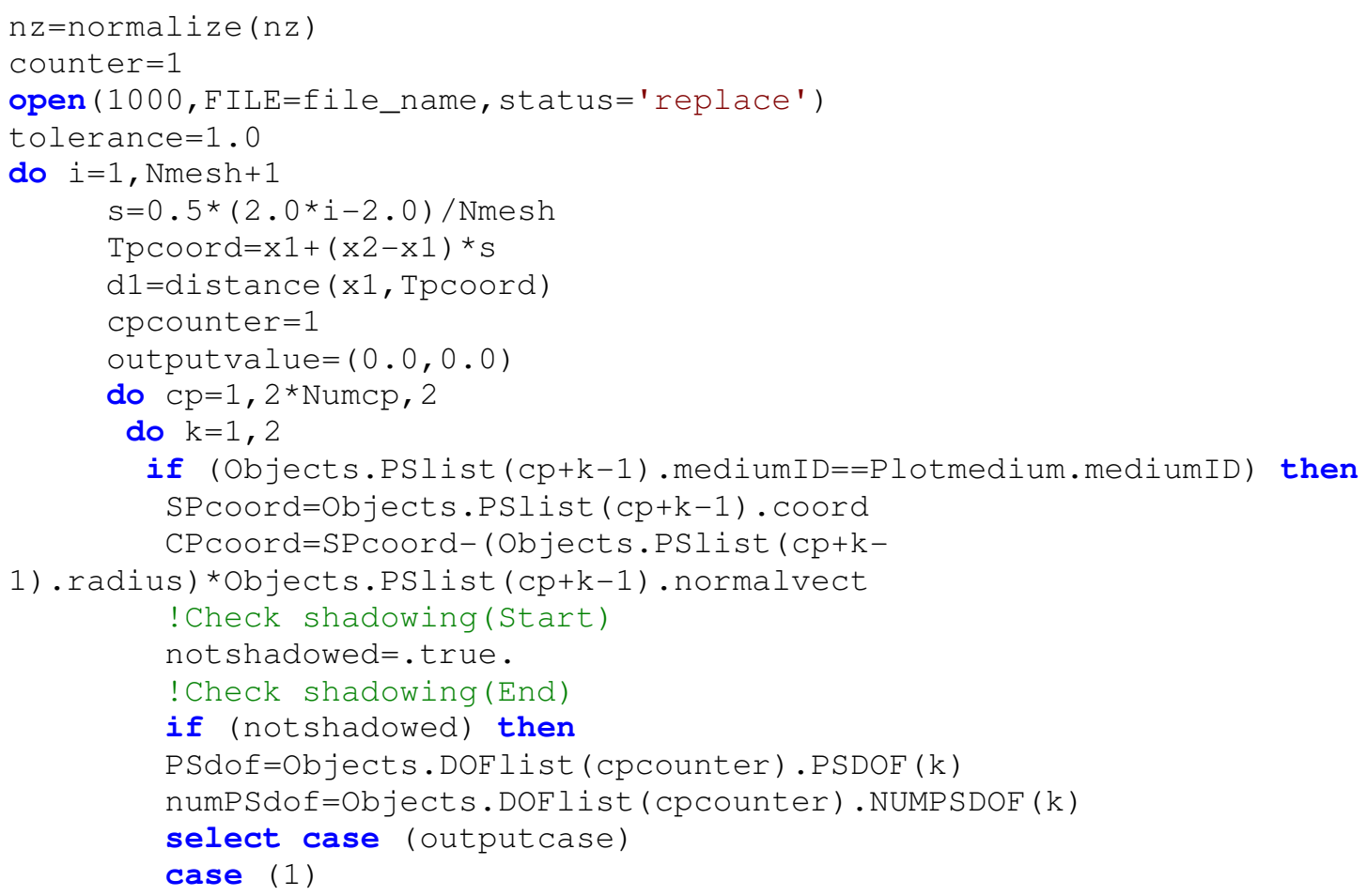

outputvalue=outputvalue+strength (PSdof) *FP (plotmedium, SPcoord, Tpcoord, o mega)

case (2)

FV2=FV(plotmedium, SPcoord, Tpcoord, omega)

outputvalue=outputvalue+strength (PSdof) *FV2 (3)

case (3)

call

SolidGreens (plotmedium, SPcoord, TPcoord, omega, G, sigma11, sigma22, sigma33, sigma12, sigma31, sigma32)

outputvalue=outputvalue+strength (PSdof) *sigma32(1)+strength (PSdof+1) * $i$ gma32(2)+\&

strength $($ PSdof +2$) * \operatorname{sigma32}(3)$

case (4)

call

SolidGreens (plotmedium, SPcoord, TPcoord, omega, G, sigma11, sigma22, sigma33, sigma12, sigma31, sigma32)

outputvalue=outputvalue+omega $($ strength (PSdof) *G $(3,1)+$ strength $(P S d o f+1)$ $\star_{\mathrm{G}}(3,2)+\varepsilon$

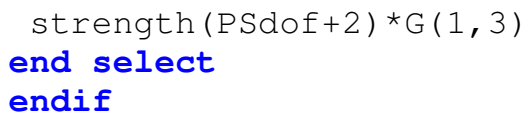




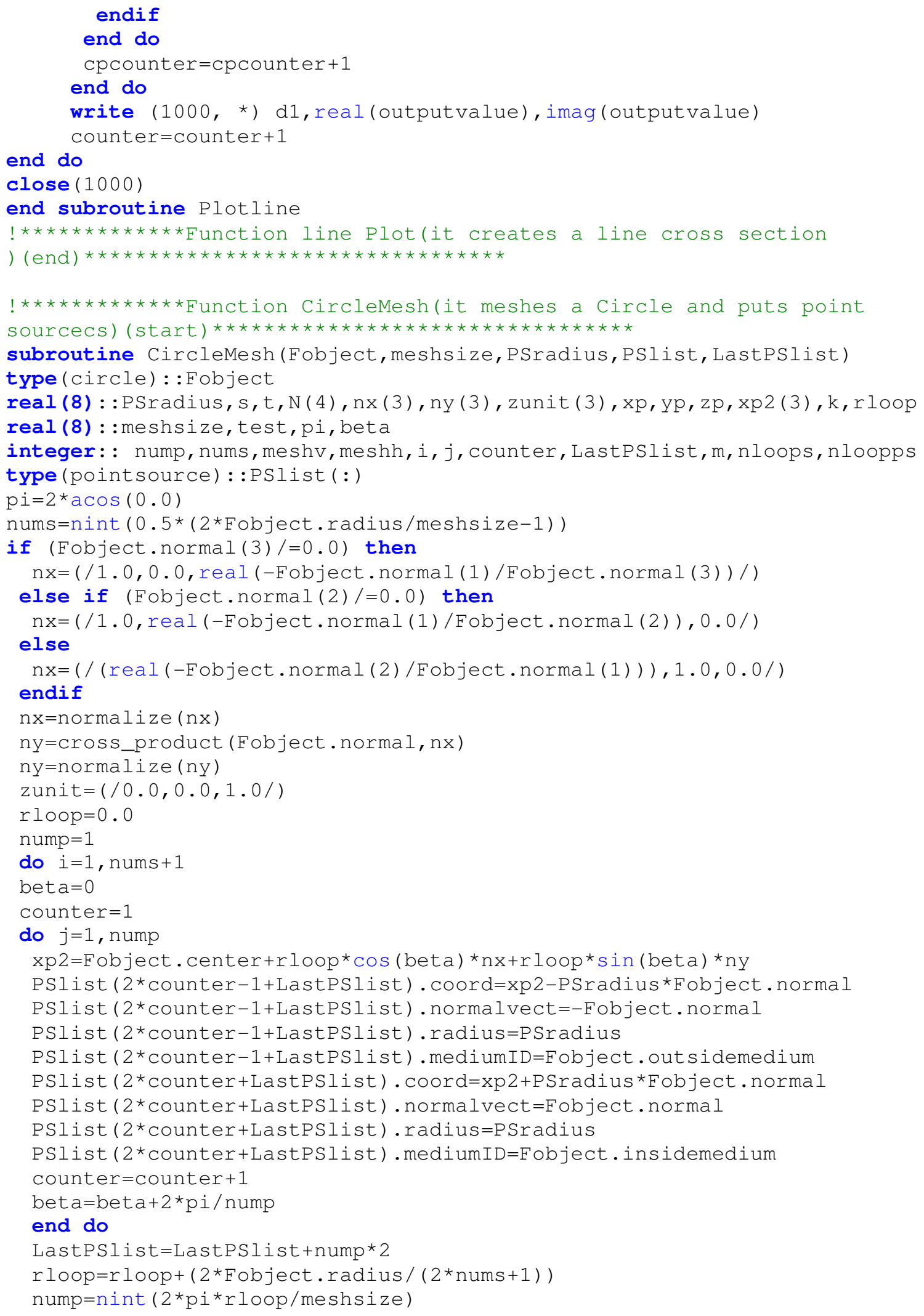


end do

end subroutine CircleMesh

! $* * * * * * * * * * * * *$ Function CircleMesh(it meshes a circle and puts point

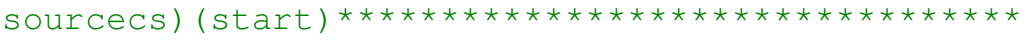

! $* \star * \star * \star * \star * \star * \star *$ Function SphereMesh(it meshes a Sphere and puts point

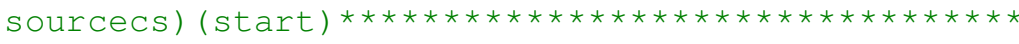

subroutine SphereMesh(Fobject, meshsize, PSradius, PSlist, LastPSlist)

type (sphere) : : Fobject

real (8) : : PSradius, xp (3), teta, beta, nvect (3)

real (8) : :meshsize, test,pi

integer:: nums, nump, $i, j$, counter, LastPslist

type (pointsource): :PSlist (:)

$\mathrm{pi}=2 * \operatorname{acos}(0.0)$

nums=nint (pi* (Fobject.radius) /(meshsize) )

teta $=0$

bet $a=0$

nump $=1$

do $i=1$, nums +1

beta $=0$

counter $=1$

do $j=1$, nump

$\mathrm{xp}=$ Fobject.radius* $\left(/\left(\sin (\right.\right.$ teta $){ }^{*} \cos ($ beta $\left.)\right),\left(\right.$ sin (teta) ${ }^{*} \sin ($ beta $\left.)\right),(\cos ($ te ta) $) /$ )

nvect $=$ normalize $(x p)$

$\mathrm{xp}=\mathrm{xp}+\mathrm{Fobject}$. center

PSlist ( $2 *$ counter $-1+$ LastPSlist) . coord=xp-PSradius*nvect

PSlist $(2 *$ counter $-1+$ LastPSlist $)$. normalvect $=-$ nvect

PSlist $(2 *$ counter $-1+$ LastPSlist) .radius $=$ PSradius

PSlist ( $2 *$ counter $-1+$ LastPSlist) . mediumID=Fobject. outsidemedium

PSlist ( $2 *$ counter+LastPSlist) . coord=xp+PSradius*nvect

PSlist $(2 *$ counter+LastPSlist) . normalvect $=$ nvect

PSlist $(2 *$ counter + LastPSlist $)$.radius $=$ PSradius

PSlist $(2 *$ counter+LastPSlist) . mediumID=Fobject. insidemedium

counter=counter +1

bet $a=$ bet $a+2 * p i /$ nump

end do

LastPSlist=LastPSlist+nump *2

teta $=$ tet $\mathrm{a}+\mathrm{pi} / \mathrm{nums}$

nump $=$ nint $(2 *$ pi*Fobject.radius*sin (teta)/meshsize)

if ( $i==$ nums) nump $=1$

end do

end subroutine SphereMesh

! $* * * * * * * * * * * * *$ Function SphereMesh(it meshes a sphere and puts point

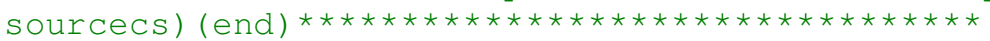

! $* \star \star \star * \star * \star * \star \star * \star *$ Function hemisphereMesh(it meshes a Semi-Sphere and puts

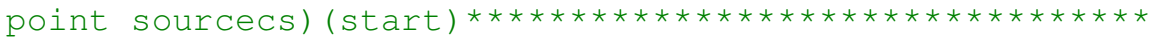

subroutine hemisphereMesh(Fobject, meshsize, PSlist, LastPSlist)

type (hemisphere) : : Fobject 


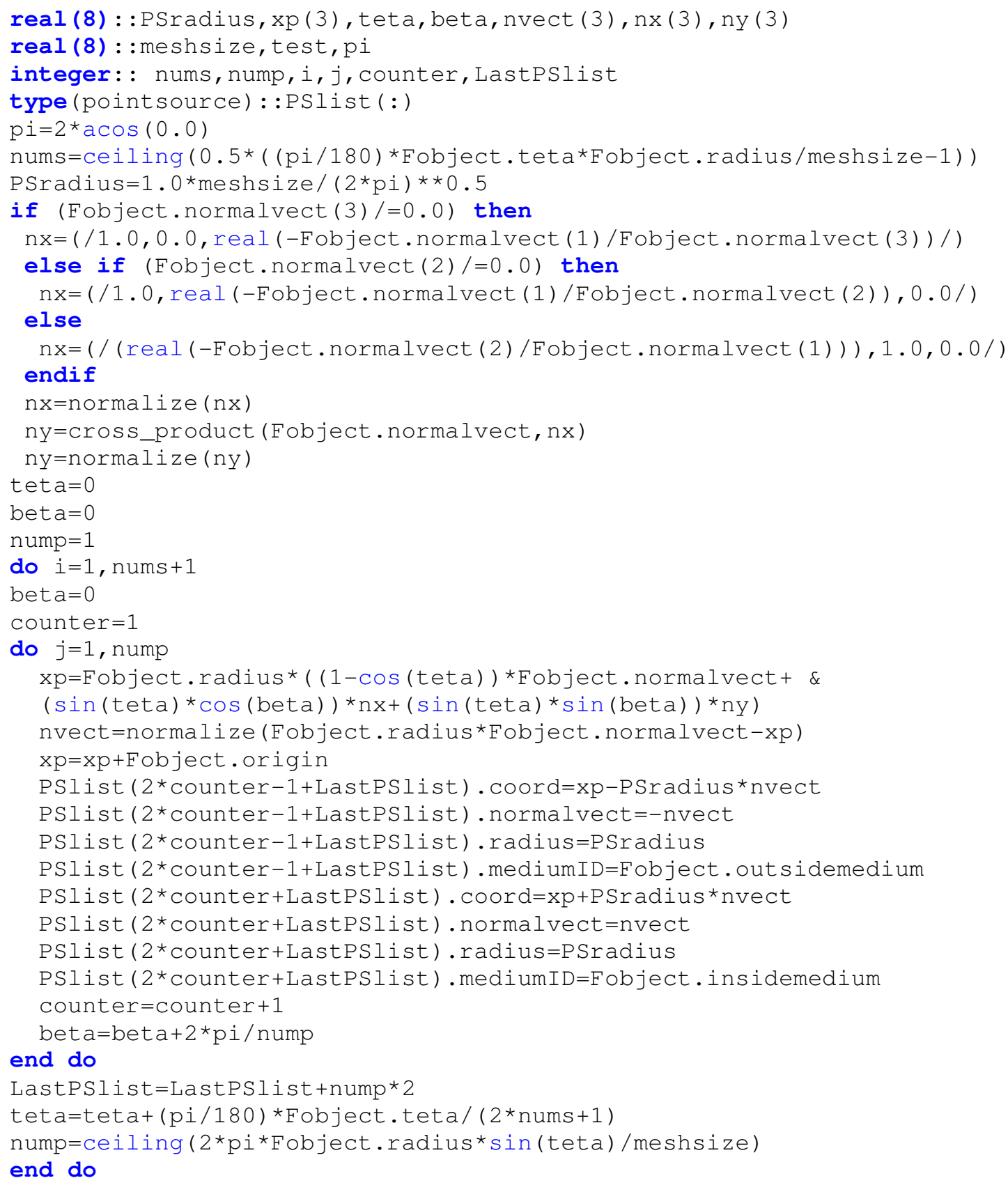




\section{"geometrylibrary" Module:}

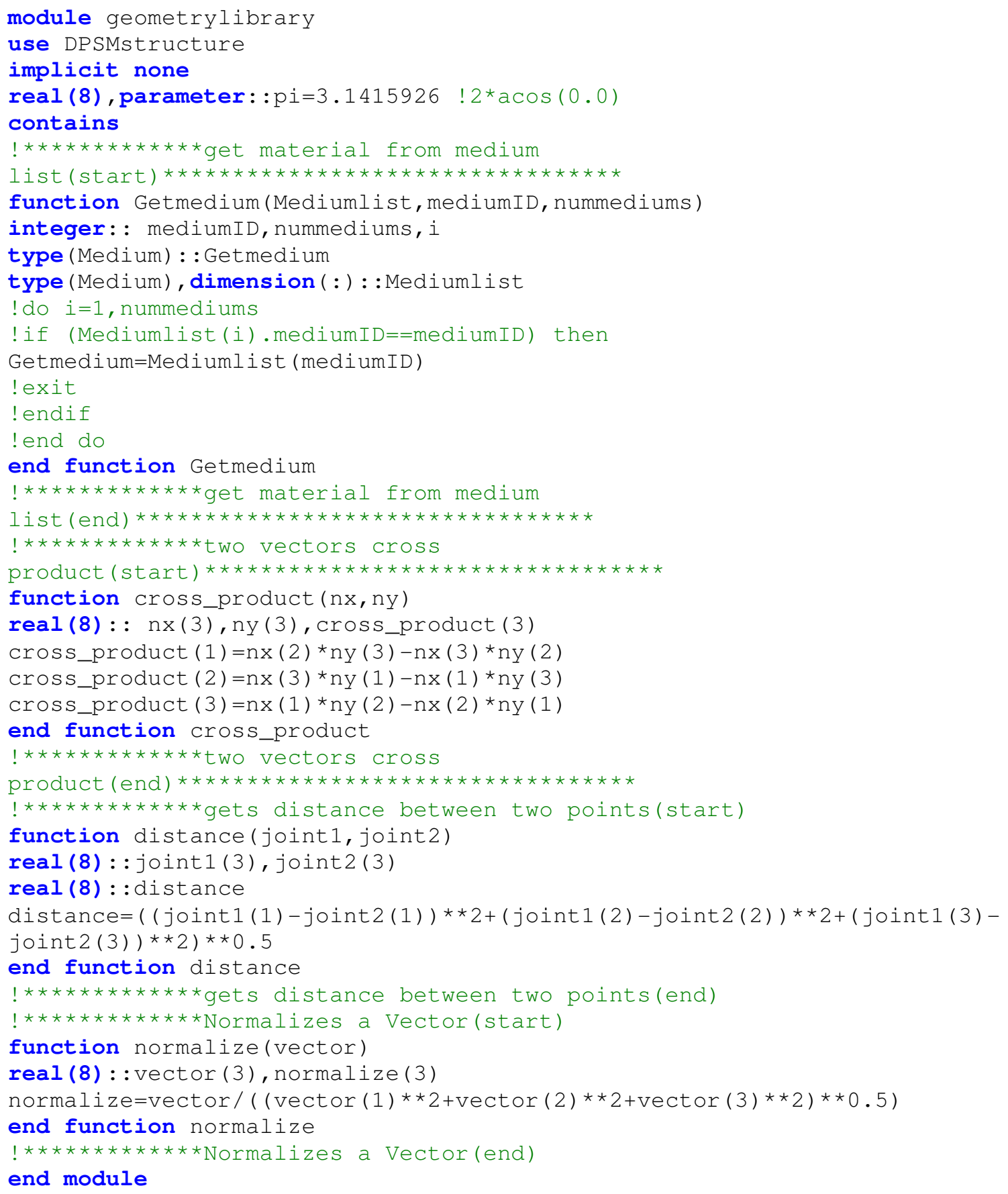




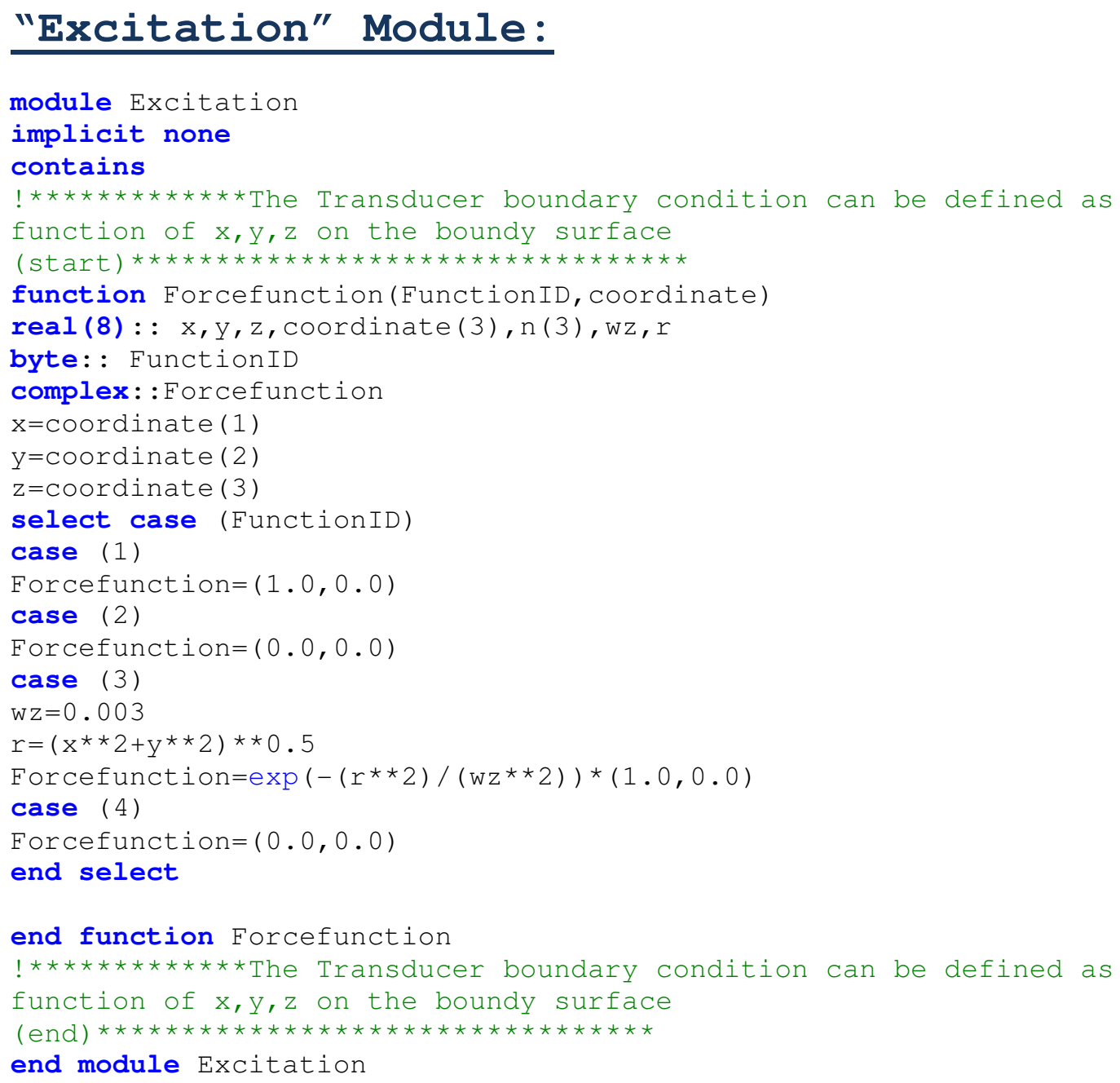




\section{"DPSMstructure" Module:}

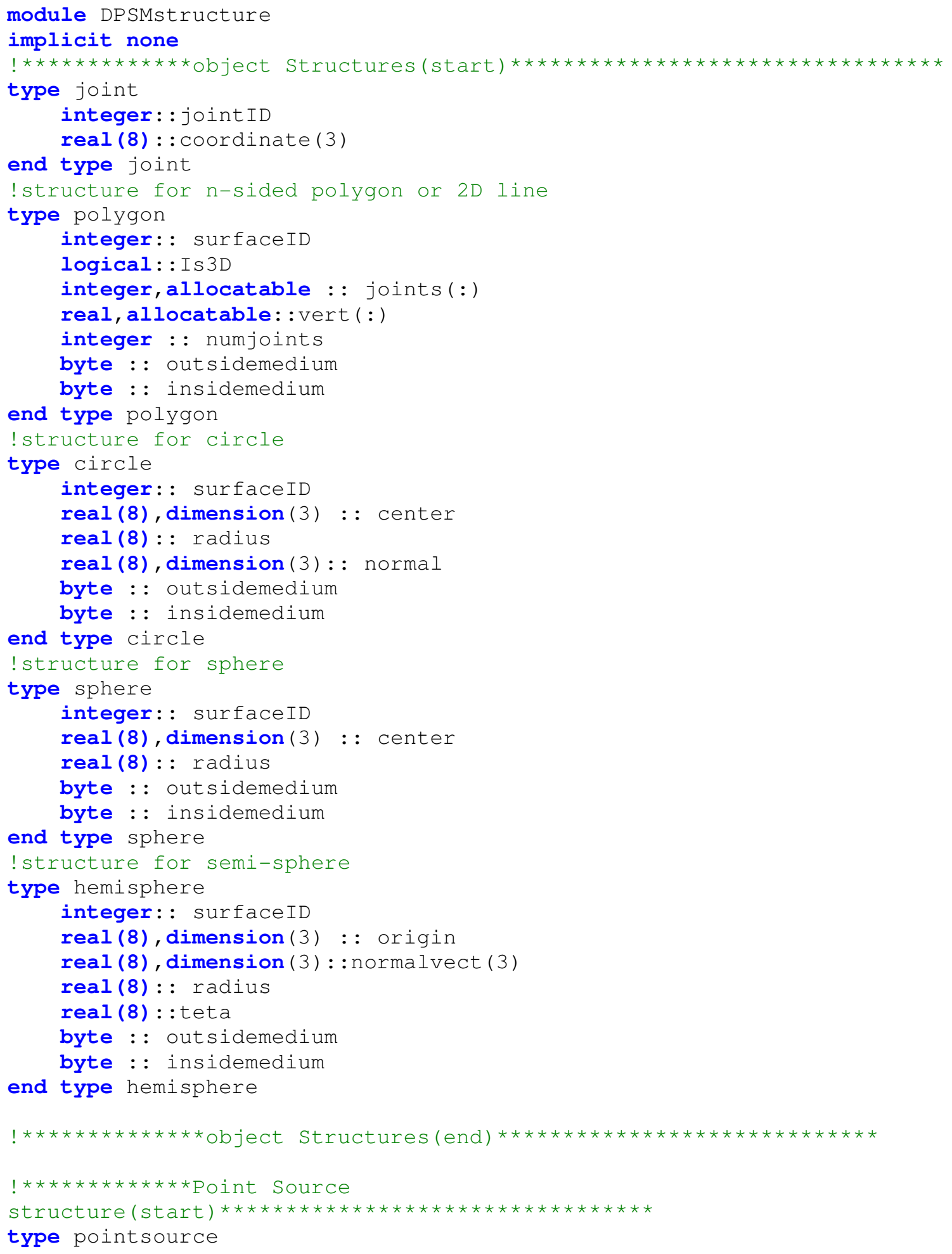




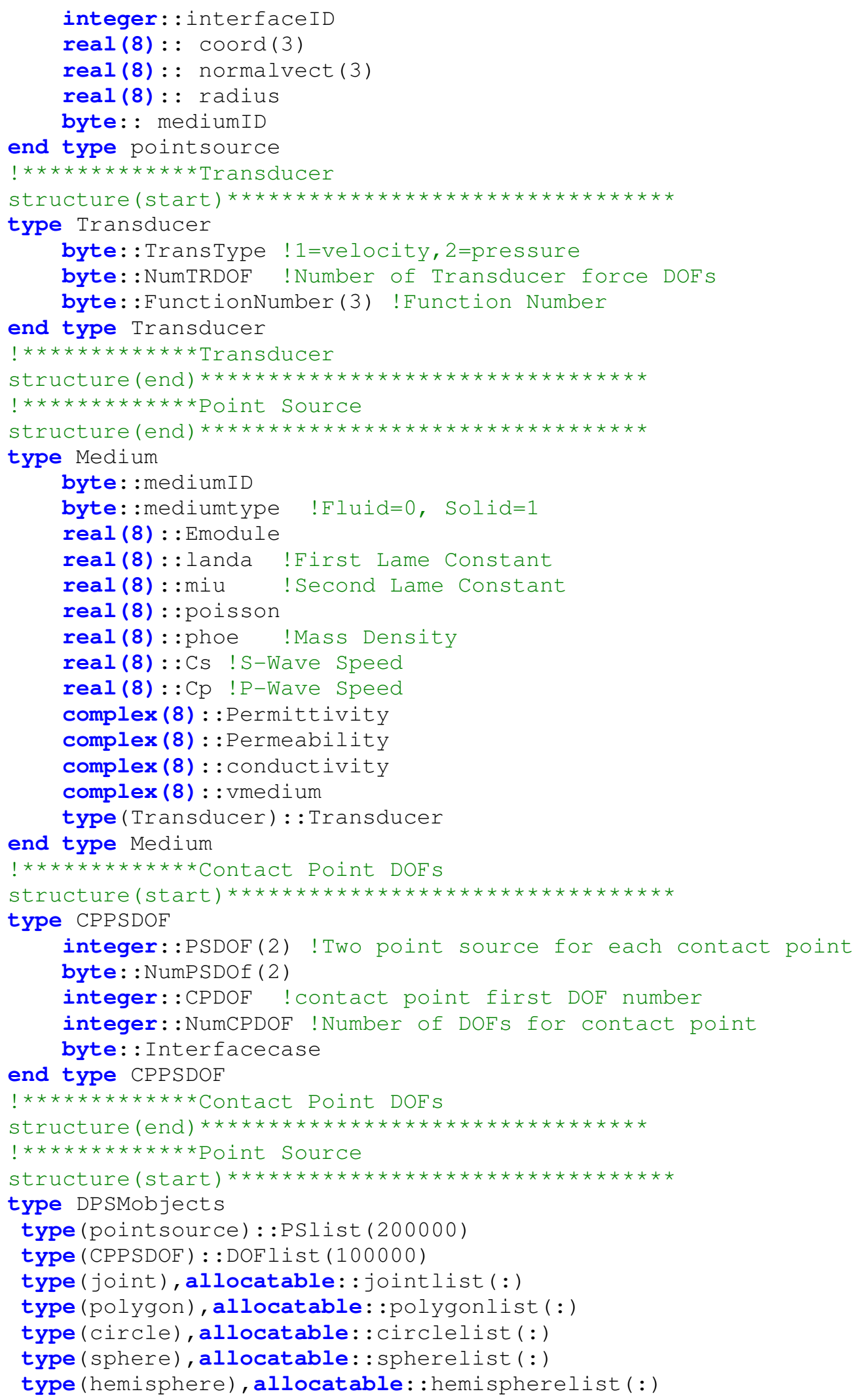


type (Medium), allocatable: : Mediumlist (:)

integer: : numpointsources

integer: : numjoints

integer: : numpolygons

integer: : numcircles

integer: : numspheres

integer: : numhemispheres

integer: : nummediums

end type DPSMobjects

end module DPSMstructure 


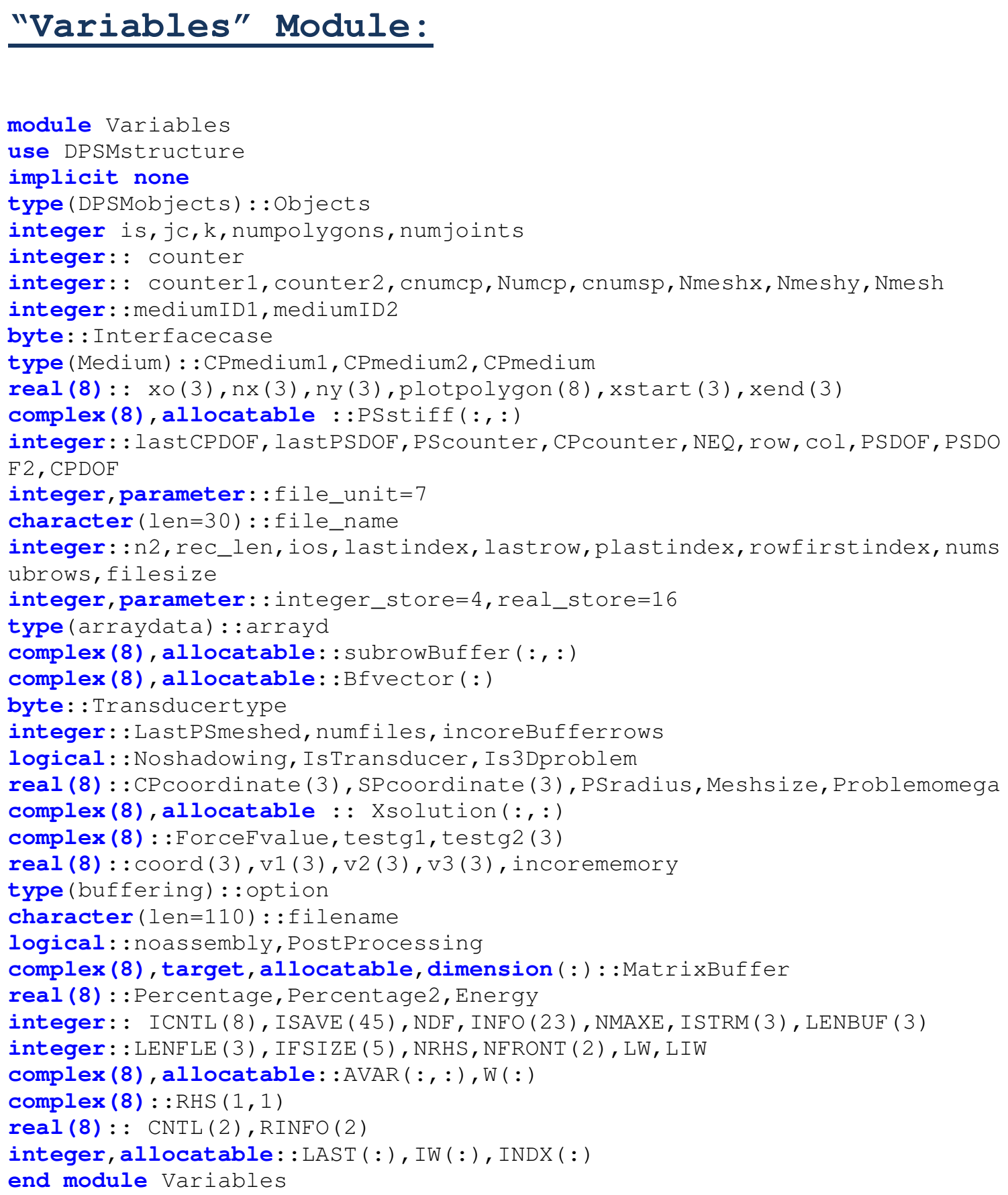




\section{LIST OF PUBLICATIONS}

Research papers generated from this investigation are listed below.

\section{Journal Papers:}

1. E. K. Rahani, T. Kundu, Z. Wu, and H. Xin, "Mechanical Damage Detection in Polymer Tiles by THz Radiation," IEEE Sensors Journal, vol. 11, pp. 1720-1725, 2011.

2. E. K. Rahani, T. Kundu, Z. Wu, and H. Xin, "Heat Induced Damage Detection by Terahertz (THz) Radiation," Journal of Infrared, Millimeter and Terahertz Waves, vol. 32, pp. 848-856, 2011.

3. E. K. Rahani and T. Kundu, "Modeling of transient ultrasonic wave propagation using Distributed Point Source Method," IEEE Transactions on Ultrasonics, Ferroelectrics and Frequency Control, vol. 58, pp. 2213-2221, 2011

4. E. K. Rahani and T. Kundu, "Gaussian-DPSM (G-DPSM) and Element Source Method (ESM) modifications to DPSM for ultrasonic field modeling," Ultrasonics, vol. 51, pp. 625-631, 2011.

5. T. Hajzargarbashi, E. K. Rahani, and T. Kundu, "Scattering of focused ultrasonic beams by two spherical cavities in close proximity," Ultrasonics, Ferroelectrics and Frequency Control, IEEE Transactions on, vol. 58, pp. 1619-1627, 2011.

6. D. Placko, T. Yanagita, E. K. Rahani, and T. Kundu, "Mesh-free modeling of the interaction between a point-focused acoustic lens and a cavity," IEEE Trans Ultrason Ferroelectr Freq Control, vol. 57, pp. 1396-404, Jun 2010.

7. T. Kundu, D. Placko, E. K. Rahani, T. Yanagita, and C. M. Dao, "Ultrasonic Field Modeling: A Comparison between Analytical, Semi-Analytical and Numerical Techniques," IEEE Transactions on Ultrasonics, Ferroelectric and Frequency Control, vol. 57, pp. 2795-2807, 2010.

\section{Book Chapters}

8. E. Kabiri Rahani, T. Kundu, "Chapter 15: THz Radiation for NDE," [Ultrasonic \& Electromagnetic NDE for Structure and Material Characterization Engineering \& Biomedical Applications], Ed. T. Kundu, Pub. CRC Press, In Press

9. T. Kundu, D. Placko, S. Das, T. Bore and E. Kabiri Rahani, "Chapter 2: Ultrasonic Field Modeling by Distributed Point Source Method", [Ultrasonic \& Electromagnetic NDE for Structure and Material Characterization - Engineering $\&$ Biomedical Applications], Ed. T. Kundu, Pub. CRC Press, In Press 


\section{Conference Papers:}

10. E. Kabiri Rahani, T. Kundu, Z. Wu, H. Xin, and K. Jata, "Mechanical and Heat Induced Damage Detection in Polymer Tiles by THz Radiation " Proceedings of the 7 th Int. Workshop on Structural Health Monitoring, Stanford Univ., CA, USA, Sept., 2009, Structural Health Monitoring 2009 - From System Integration to Autonomous Systems, Ed. F-K. Chang, Pub. DEStech Publications Inc. pp. 471-478, 2009.

11. E. Kabiri Rahani and T. kundu, "Gaussian-DPSM a Modification on Conventional Distributed Point Source Method (DPSM) for Ultrasonic Field Modeling," Proceedings of the 3rd Asia Pacific Workshop on Structural Health Monitoring , Tokyo, Japan, Nov. 30 to Dec. 2, 2010.

12. E. Kabiri Rahani, T. Hajzargarbashi, and T. Kundu, "Distributed Point Source Method and its Applications in Solving Acoustic Wave Scattering Problems," in Proceedings of Meetings on Acoustics (POMA), 162nd meeting of the acoustical society of America, San Diego, Oct. 31-Nov.4, 2011. 


\section{REFERENCES}

[1] Y.-S. Lee, Principles of terahertz science and technology, 1st ed. New York, NY: Springer, 2008.

[2] W. contributors. Terahertz radiation. Available: http://en.wikipedia.org/w/index.php?title=Terahertz_radiation\&oldid=435149551

[3] A. R. Sanchez, N. Karpowicz, J. Xu, and X. C. Zhang, "Damage and defect inspection with terahertz waves," in The 4th International Workshop on Ultrasonic and Advanced Methods for Nondestructive Testing and Material Characterization, UMass Dartmouth, N. Dartmouth, 2006.

[4] C. D. Stoik, M. J. Bohn, and J. L. Blackshire, "Nondestructive evaluation of aircraft composites using transmissive terahertz time domain spectroscopy," Opt. Express, vol. 16, pp. 17039-17051, 2008.

[5] G. Z. Zhao, M. ter Mors, T. Wenckebach, and P. C. M. Planken, "Terahertz dielectric properties of polystyrene foam," Journal of the Optical Society of America B-Optical Physics, vol. 19, pp. 1476-1479, Jun 2002.

[6] R. Piesiewicz, C. Jansen, S. Wietzke, D. Mittleman, M. Koch, and T. Kürner, "Properties of Building and Plastic Materials in the THz Range," International Journal of Infrared and Millimeter Waves, vol. 28, pp. 363-371, 2007.

[7] H. Zhong, J. Z. Xu, X. Xie, T. Yuan, R. Reightler, E. Madaras, and X. C. Zhang, "Nondestructive defect identification with terahertz time-of-flight tomography," Ieee Sensors Journal, vol. 5, pp. 203-208, Apr 2005.

[8] H. Zhong, N. Karpowicz, J. Xu, Y. Deng, W. Ussery, M. Shur, and X.-C. Zhang, "Detection of space shuttle insulation foam defects by using a $0.2 \mathrm{THz}$ Gunn diode oscillator and pyroelectric detector," 2004, p. FTuG28.

[9] D. M. Mittleman, S. Hunsche, L. Boivin, and M. C. Nuss, "T-ray tomography," Optics Letters, vol. 22, pp. 904-906, Jun 151997.

[10] N. Karpowicz, H. Zhong, C. L. Zhang, K. I. Lin, J. S. Hwang, J. Z. Xu, and X. C. Zhang, "Compact continuous-wave subterahertz system for inspection applications," Applied Physics Letters, vol. 86, pp. -, Jan 312005.

[11] C. Zhang, K. Mu, X. Jiang, Y. Jiao, L. Zhang, Q. Zhou, Y. Zhang, J. Shen, G. Zhao, and X. C. Zhang, "Identification of explosives and drugs and inspection of material defects with $\mathrm{THz}$ radiation," in Proceedings of SPIE The International Society For Optical Engineering, Beijing, China, 2007, pp. 68400S-10.

[12] J. C. Aldrin, D. J. Roth, J. P. Seebo, and W. P. Winfree, "Protocol and Assessment of Signal Processing and Feature Extraction Methods for Terahertz NDE for Spray-On Foam Insulation," AIP Conference Proceedings, vol. 894, pp. 432-439, 2007.

[13] H. T. Banks and N. L. Gibson, "Void detection in foam with knit lines using THz pulse interrogation," Mathematical and Computer Modelling, vol. 44, pp. 807$815,2006$. 
[14] R. F. Anastasi and E. I. Madaras, "Terahertz NDE for Under Paint Corrosion Detection and Evaluation," AIP Conference Proceedings, vol. 820, pp. 515-522, 2006.

[15] Y. Oyama, L. Zhen, T. Tanabe, and M. Kagaya, "Sub-terahertz imaging of defects in building blocks," NDT \& E International, vol. 42, pp. 28-33, 2009.

[16] J. W. Lamb, "Miscellaneous data on materials for millimetre and submillimetre optics," International Journal of Infrared and Millimeter Waves, vol. 17, pp. 1997-2034, 1996.

[17] L. Duvillaret, F. Garet, and J. L. Coutaz, "A reliable method for extraction of material parameters in terahertz time-domain spectroscopy," Ieee Journal of Selected Topics in Quantum Electronics, vol. 2, pp. 739-746, Sep 1996.

[18] T. D. Dorney, R. G. Baraniuk, and D. M. Mittleman, "Material parameter estimation with terahertz time-domain spectroscopy," Journal of the Optical Society of America a-Optics Image Science and Vision, vol. 18, pp. 1562-1571, Jul 2001.

[19] Z. Wu, L. Wang, Y. Peng, A. Young, S. Seraphin, and H. Xin, "Terahertz characterization of multi-walled carbon nanotube films," Journal of Applied Physics, vol. 103, pp. 094324-094324-6, 2008.

[20] E. K. Rahani, T. Kundu, Z. Wu, and H. Xin, "Heat Induced Damage Detection by Terahertz (THz) Radiation," Journal of Infrared, Millimeter and Terahertz Waves, vol. 32, pp. 848-856, 2011.

[21] E. K. Rahani, T. Kundu, Z. Wu, and H. Xin, "Mechanical Damage Detection in Polymer Tiles by THz Radiation," IEEE Sensors Journal, vol. 11, pp. 1720-1725, 2011.

[22] P. U. Jepsen, R. H. Jacobsen, and S. R. Keiding, "Generation and detection of terahertz pulses from biased semiconductor antennas," J. Opt. Soc. Am. B, vol. 13, pp. 2424-2436, 1996.

[23] D. K. Cheng, Field and wave electromagnetics. Reading, Mass.: Addison Wesley, 1983.

[24] T. Hoshina, H. Yasuno, H. Kakemoto, T. Tsurumi, and S. Wada, "Particle Size and Temperature Dependence of THz-Region Dielectric Properties for BaTiO<sub $>3</$ sub $>$ Nanoparticles," Ferroelectrics, vol. 353, pp. 55-62, 2007.

[25] D. Stroud, "Generalized effective-medium approach to the conductivity of an inhomogeneous material," Physical Review B, vol. 12, p. 3368, 1975.

[26] D. Placko and T. Kundu, Eds., DPSM for Modeling Engineering Problems: John Wiley \& Sons, Inc., 2007.

[27] D. Placko and T. Kundu, "Modeling of Ultrasonic Field by Distributed Point Source Method," in Ultrasonic Nondestructive Evaluation, T. Kundu, Ed., ed: CRC Press, 2004, pp. 143-202.

[28] T. Kundu, D. Placko, E. K. Rahani, T. Yanagita, and C. M. Dao, "Ultrasonic Field Modeling: A Comparison between Analytical, Semi-Analytical and Numerical Techniques," IEEE Transactions on Ultrasonics, Ferroelectric and Frequency Control, vol. 57, pp. 2795-2807, 2010. 
[29] S. Banerjee, T. Kundu, and D. Placko, "Ultrasonic Field Modeling in Multilayered Fluid Structures Using the Distributed Point Source Method Technique," Journal of Applied Mechanics, vol. 73, pp. 598-609, 2006.

[30] S. Banerjee and T. Kundu, "Elastic wave field computation in multilayered nonplanar solid structures: A mesh-free semianalytical approach," The Journal of the Acoustical Society of America, vol. 123, pp. 1371-1382, 2008.

[31] S. Das, S. Banerjee, and T. Kundu, "Elastic wave scattering in a solid half-space with a circular cylindrical hole using the Distributed Point Source Method," International journal of solids and structures., vol. 45, p. 4498, 2008.

[32] A. Shelke, S. Das, and T. Kundu, "Distributed Point Source Method for Modeling Scattered Ultrasonic Fields in Presence of an Elliptical Cavity," Structural Health Monitoring, vol. 9, pp. 527-539, 2010.

[33] F. M. Kahnert, "Numerical methods in electromagnetic scattering theory," Journal of Quantitative Spectroscopy and Radiative Transfer, vol. 79-80, pp. 775824.

[34] D. I. Kaklamani and H. T. Anastassiu, "Aspects of the Method of Auxiliary Sources (MAS) in computational electromagnetics," Antennas and Propagation Magazine, IEEE, vol. 44, pp. 48-64, 2002.

[35] P. V. Zinin and W. Weise, "Theory and applications of acoustic microscopy," in Ultrasonic Nondestructive Evaluation: Engineering and Biological Material Characterization, T. Kundu, Ed., ed Boca Raton, FL: CRC Press, 2004, pp. 654724.

[36] S.-Y. Sung and Y.-G. Lee, "Trapping of a micro-bubble by non-paraxial Gaussian beam: computation using the FDTD method," Opt. Express, vol. 16, pp. 34633473, 2008.

[37] V. Volski and G. A. E. Vandenbosch, "Modeling of a cavity filled with a plane multilayered dielectric using the method of auxiliary sources," Microwave Theory and Techniques, IEEE Transactions on, vol. 54, pp. 235-239, 2006.

[38] W. contributors. Gaussian beam. Available: http://en.wikipedia.org/w/index.php?title=Gaussian_beam\&oldid=457979467

[39] B. E. A. Saleh and M. C. Teich, "Beam Optics," in Fundamentals of photonics, ed New York: Wiley, 1991, pp. 80-107.

[40] W. Sun, S. Pan, and Y. Jiang, "Computation of the optical trapping force on small particles illuminated with a focused light beam using a FDTD method," Journal of Modern Optics, vol. 53, pp. 2691 - 2700, 2006.

[41] R. Ahmad, T. Kundu, and D. Placko, "Modeling of phased array transducers," $J$ Acoust Soc Am, vol. 117, pp. 1762-76, Apr 2005.

[42] S. Banerjee and T. Kundu, "Elastic wave propagation in sinusoidally corrugated waveguides," The Journal of the Acoustical Society of America, vol. 119, pp. 2006-2017, 2006.

[43] S. Das, C. M. Dao, S. Banerjee, and T. Kundu, "DPSM Modeling for Studying Interaction Between Bounded Ultrasonic Beams and Corrugated Plates with Experimental Verification," Ultrasonics, Ferroelectrics and Frequency Control, IEEE Transactions on, vol. 54, pp. 1860-1872, 2007. 
[44] S. Banerjee and T. Kundu, "Semi-analytical modeling of ultrasonic fields in solids with internal anomalies immersed in a fluid," Wave Motion, vol. 45, pp. 581-595, 2008.

[45] J. Lubbers and J. van den Berg, "An ultrasonic detector for microgasemboli in a bloodflow line," Ultrasound in medicine \& biology, vol. 2, pp. 301-310, 1977.

[46] K. Hatteland and B. K. H. Semb, "Gas Bubble Detection in Fluid Lines by Means of Pulsed Doppler Ultrasound," Scandinavian Cardiovascular Journal, vol. 19, pp. 119-123, 1985.

[47] I. Ihara, C.-K. Jen, and D. R. Franca, "Detection of Inclusion in Molten Metal by Focused Ultrasonic Wave," Japanese Journal of Applied Physics, vol. 39, pp. 3152-3153, 2000.

[48] O. I. Lobkis, T. Kundu, and P. V. Zinin, "A theoretical analysis of acoustic microscopy of spherical cavities," Wave Motion, vol. 21, pp. 183-201, 1995.

[49] D. Placko, T. Yanagita, E. K. Rahani, and T. Kundu, "Mesh-free modeling of the interaction between a point-focused acoustic lens and a cavity," IEEE Trans Ultrason Ferroelectr Freq Control, vol. 57, pp. 1396-404, Jun 2010.

[50] T. Hajzargarbashi, E. K. Rahani, and T. Kundu, "Scattering of focused ultrasonic beams by two spherical cavities in close proximity," IEEE Transactions on Ultrasonics, Ferroelectrics and Frequency Control, In Press.

[51] J. D. Achenbach, Wave Propagation in Elastic Solids: Amsterdam: North Holland, 1973.

[52] K. F. Graff, Wave Motion in Elastic Solids: Columbus, Ohio: Ohio State University, 1975.

[53] J. Miklowitz, The Theory of Elastic Waves and Waveguides: Amsterdam: North Holland, 1978.

[54] W. Lord, R. Ludwig, and Z. You, "Developments in ultrasonic modeling with finite element analysis," Journal of Nondestructive Evaluation, vol. 9, pp. 129143, 1990.

[55] R. Ludwig, D. Moore, and W. Lord, "A comparative study of analytical and numerical transient force excitations on an elastic half-space," Ultrasonics, Ferroelectrics and Frequency Control, IEEE Transactions on, vol. 36, pp. 342350, 1989.

[56] Z. You, M. Lusk, R. Ludwig, and W. Lord, "Numerical simulation of ultrasonic wave propagation in anisotropic and attenuative solid materials," Ultrasonics, Ferroelectrics and Frequency Control, IEEE Transactions on, vol. 38, pp. 436445, 1991.

[57] P. Kudela, M. Krawczuk, and W. Ostachowicz, "Wave propagation modelling in 1D structures using spectral finite elements," Journal of Sound and Vibration, vol. 300, pp. 88-100, 2007.

[58] M. Mitra and S. Gopalakrishnan, "Wavelet Based Spectral Finite Element Modelling and Detection of De-Lamination in Composite Beams," Proceedings: Mathematical, Physical and Engineering Sciences, vol. 462, pp. 1721-1740, 2006. 
[59] M. Mitra and S. Gopalakrishnan, "Wavelet Based 2-D spectral finite element formulation for wave propagation analysis in isotropic plates," Computer Modeling In Engineering \& Science, vol. 15, pp. 49-68, 2006.

[60] H. Antes, "A boundary element procedure for transient wave propagations in twodimensional isotropic elastic media," Finite Elements in Analysis and Design, vol. 1, pp. 313-322, 1985.

[61] L. Gaul and M. Schanz, "A comparative study of three boundary element approaches to calculate the transient response of viscoelastic solids with unbounded domains," Computer Methods in Applied Mechanics and Engineering, vol. 179, pp. 111-123, 1999.

[62] T. Kundu, D. Placko, S. Das, T. Bore, and E. K. Rahani, "Modeling of Ultrasonic Fields by Distributed Point Source Method (DPSM)," in Ultrasonic \& Electromagnetic NDE for Structure and Material Characterization - Engineering \& Biomedical Applications, T. Kundu, Ed., ed: CRC Press, Taylor and Francis, 2011.

[63] S. Banerjee, T. Kundu, and N. A. Alnuaimi, "DPSM technique for ultrasonic field modelling near fluid-solid interface," Ultrasonics, vol. 46, pp. 235-250, 2007.

[64] T. Yanagita, T. Kundu, and D. Placko, "Ultrasonic field modeling by distributed point source method for different transducer boundary conditions," J Acoust Soc Am, vol. 126, pp. 2331-9, Nov 2009.

[65] J. Cheng, W. Lin, and Y.-X. Qin, "Extension of the distributed point source method for ultrasonic field modeling," Ultrasonics, vol. 51, pp. 571-580, 2011.

[66] D. Placko, T. Yanagita, E. K. Rahani, and T. Kundu, "Mesh-Free Modeling of the Interaction Between a Point-Focused Acoustic Lens and a Cavity," IEEE Transactions on Ultrasonics, Ferroelectrics and Frequency Control, vol. 57, p. 1396, 2010.

[67] M. Eskandarzade, T. Kundu, N. Liebeaux, D. Placko, and F. Mobadersani, "Numerical simulation of electromagnetic acoustic transducers using distributed point source method," Ultrasonics, vol. 50, pp. 583-591, 2010.

[68] P. K. Banerjee, The boundary element methods in engineering, 2nd ed. London ; New York: McGraw-Hill, 1994.

[69] J. Cheng, J.-y. Lu, W. Lin, and Y.-X. Qin, "A new algorithm for spatial impulse response of rectangular planar transducers," Ultrasonics, vol. 51, pp. 229-237, 2011.

[70] H. Djelouah, J. C. Baboux, and M. Perdrix, "Theoretical and experimental study of the field radiated by ultrasonic focussed transducers," Ultrasonics, vol. 29, pp. 188-200, 1991.

[71] T. Imamura, "Specific acoustic impedance of the ultrasonic field by the circular flat transducers," Ultrasonics, vol. 36, pp. 751-757, 1998.

[72] S. Yi, S. F. Ling, and M. Ying, "Time-domain analyses of acoustics--structure interactions for piezoelectric transducers," The Journal of the Acoustical Society of America, vol. 109, pp. 2762-2770, 2001.

[73] L. W. Schmerr, Fundamental of Ultrasonic Nondestructive Evaluation:A Modeling Approach Springer, 1998. 
[74] G. Beer, C. Duenser, I. M. Smith, and SpringerLink (Online service). (2008). The boundary element method with programming for engineers and scientists. Available: http://dx.doi.org/10.1007/978-3-211-71576-5

[75] A. H. Stroud and D. Secrest, Gaussian Quadrature Formulas Prentice-Hall, Englewood Cliffs, 1966.

[76] S. M. Hasheminejad, "Elastic Wave Scattering by Two Spherical Inclusions in a Poroelastic Medium," J. Eng. Mech., vol. 131, p. 953, 2005.

[77] S. Banerjee and T. Kundu, "Modeling ultrasonic wave scattering by internal anomalies for structural monitoring," in SPIE Newsroom, ed, 2007.

[78] B. Tittmann, "Scattering of longitudinal waves inc10.1121/1.381697 on a spherical cavity in a solid," J. Acoust. Soc. Am., vol. 63, p. 68, 1978.

[79] B. R. Tittmann and E. Richard Cohen, "Analysis of ultrasonic wave scattering for characterization of defects in solids: I. Spherical inclusions and reciprocity," Journal of Nondestructive Evaluation, vol. 3, pp. 175-182, 1982.

[80] I. Ihara, C.-K. Jen, Fran, ccedil, and D. R. a, "Detection of Inclusion in Molten Metal by Focused Ultrasonic Wave," Japanese Journal of Applied Physics, vol. 39, p. 3152, 2000.

[81] I. Ihara, "C-scan imaging in molten zinc by focused ultrasonic waves," J. Acoust. Soc. Am., vol. 107, p. 1042, 2000.

[82] I. Ihara, C. K. Jen, and D. R. Franca, "Materials evaluation using long clad buffer rods," in Ultrasonics Symposium, 1998. Proceedings., 1998 IEEE, 1998, pp. 803807 vol.1.

[83] O. I. Lobkis and P. V. Zinin, "Acoustic Microscopy of Spherical Objects Theoretical Approach," Acoustic Letters, vol. 14, pp. 168-172, 1990.

[84] P. V. Zinin, W. Weise, O. Lobkis, O. Kolosov, and S. Boseck, "Fourier optics analysis of spherical particles image formation in reflection acoustic microscopy," Optik, vol. 98, pp. 45-60, 1994.

[85] T. Hajzargarbashi, E. K. Rahani, and T. Kundu, "Scattering of focused ultrasonic beams by two spherical cavities in close proximity," Ultrasonics, Ferroelectrics and Frequency Control, IEEE Transactions on, vol. 58, pp. 1619-1627, 2011.

[86] T. Kundu, D. Placko, T. Yanagita, and S. Sathish, Micro Interferometric Acoustic Lens: Mesh-Free Modeling with Experimental Verification vol. 7295. Bellingham, WA, ETATS-UNIS: Society of Photo-Optical Instrumentation Engineers, 2009.

[87] E. K. Rahani and T. Kundu, "Modeling of transient ultrasonic wave propagation using Distributed Point Source Method," IEEE Transactions on Ultrasonics, Ferroelectrics and Frequency Control, vol. 58, pp. 2213-2221, 2011.

[88] "COMSOL Multiphysics Version 3.5a," ed: COMSOL AB, 2008, p. User's Guide.

[89] J. Zhu and A. H. Shah, "A Hybrid method for transient wave scattering by flaws in composite plates," International Journal of Solids and Structures, vol. 34, pp. 1719-1734, 1997.

[90] E. k. Rahani, T. Hajzargarbashi, and T. Kundu, "Distributed Point Source Method and its Applications in Solving Acoustic Wave Scattering Problems," in 
Proceedings of Meetings on Acoustics (POMA), 162nd meeting of the acoustical society of America, San Diego, 2011. 